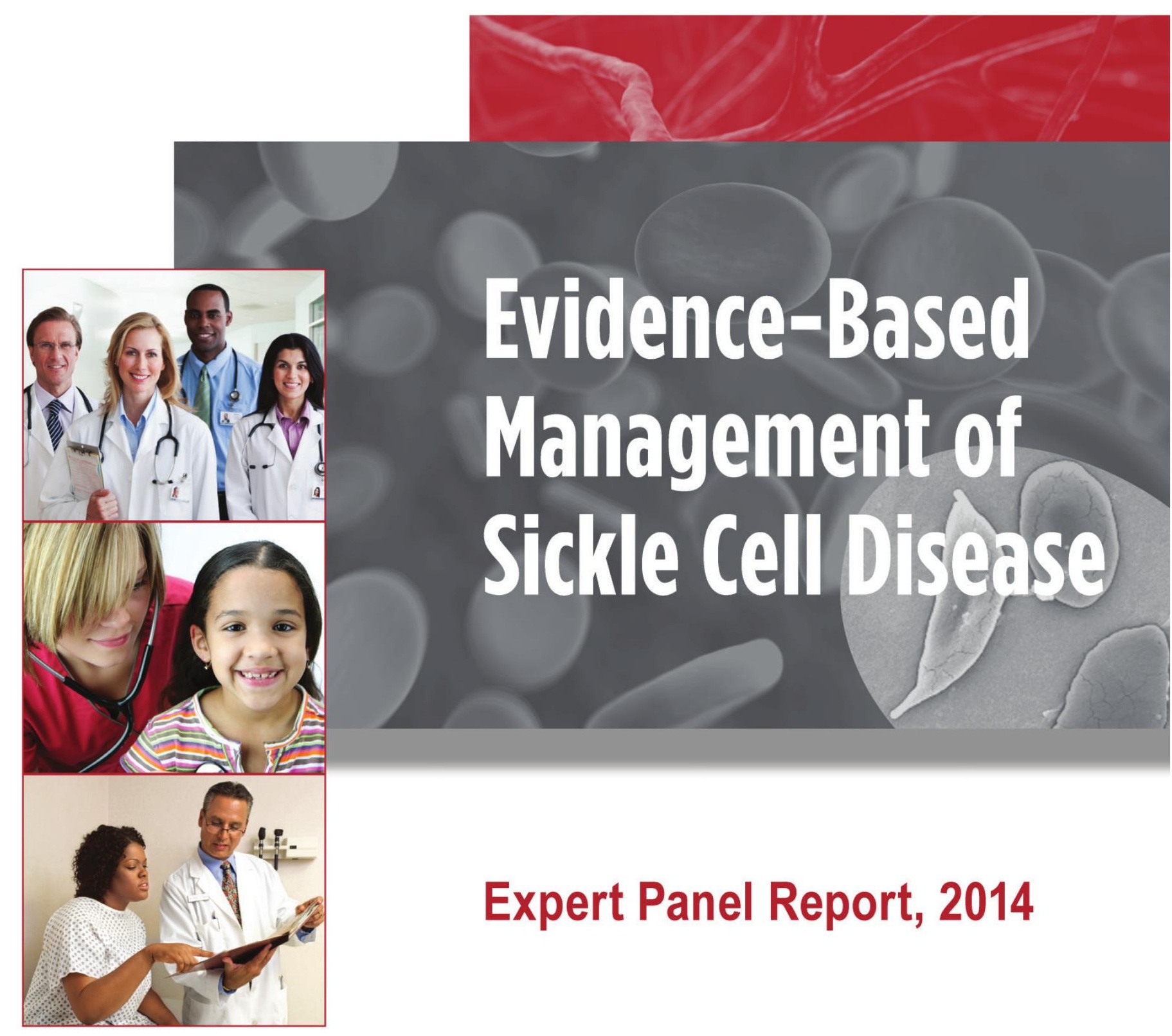





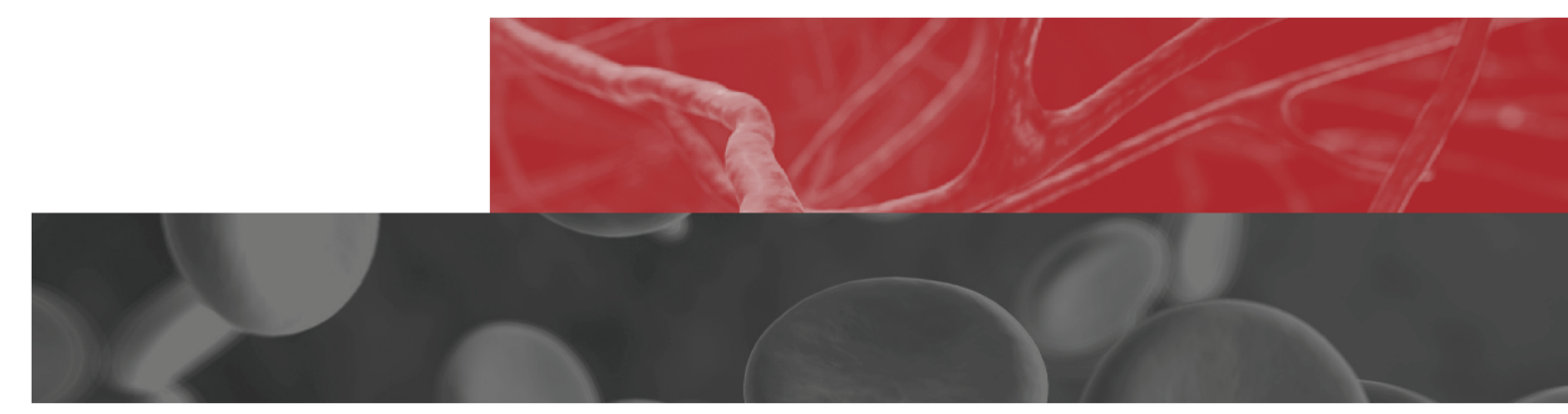

\section{Evidence-Based Management of Sickle Cell Disease}

\section{Expert Panel Report, 2014}

U.S. Department of Health and Human Services National Institutes of Health

National Heart, Lung, and Blood Institute 



\section{Contents}

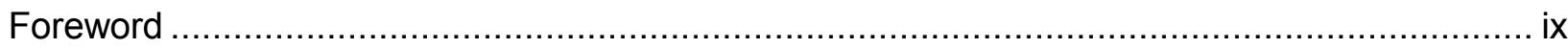

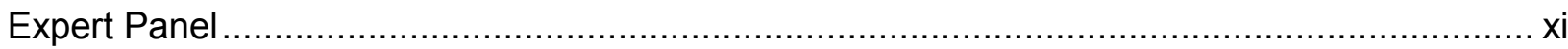

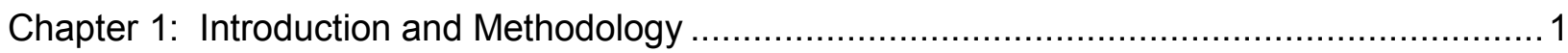

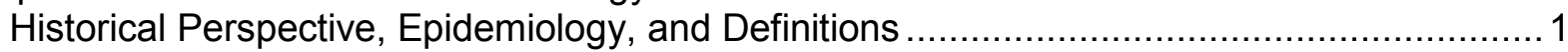

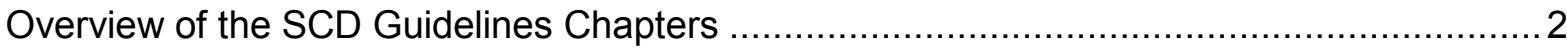

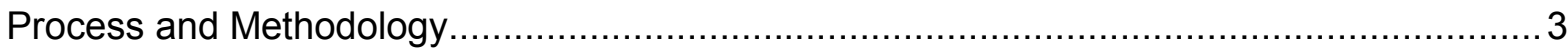

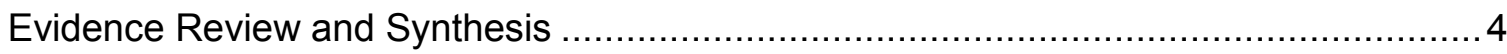

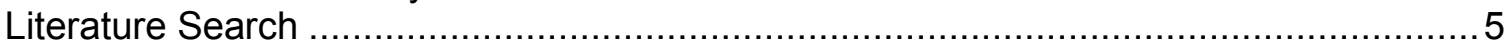

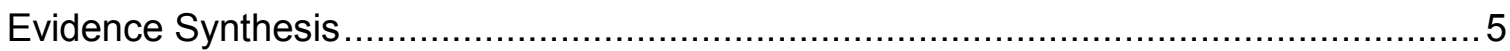

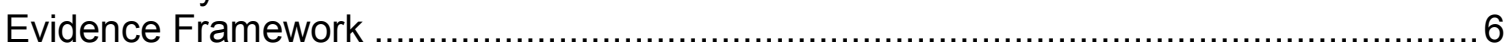

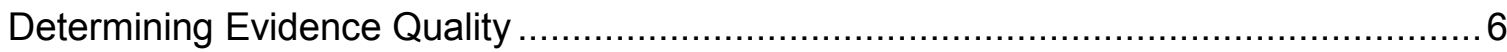

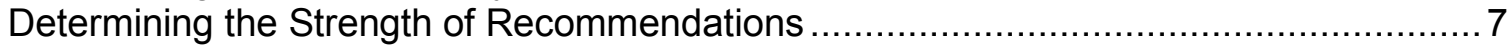

Existing Systematic Reviews and Clinical Practice Guidelines ............................... 8

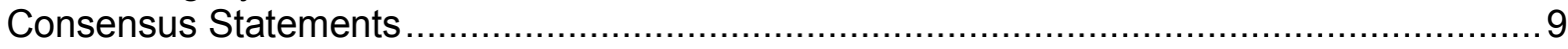

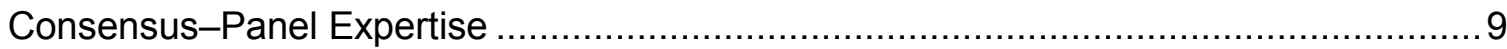

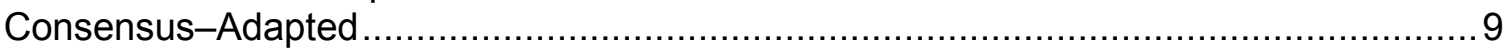

Clinical Practice Guidelines and the Institute of Medicine ....................................... 10

Chapter 2: Health Maintenance for People With Sickle Cell Disease ................................. 11

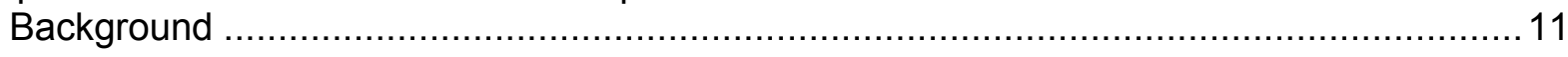

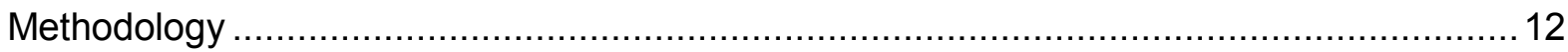

Prevention of Invasive Pneumococcal Infection ............................................... 12

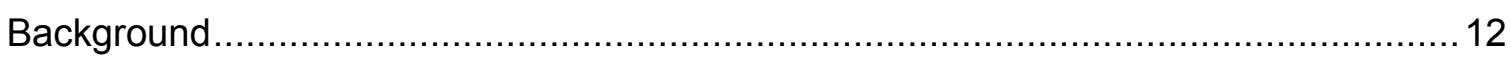

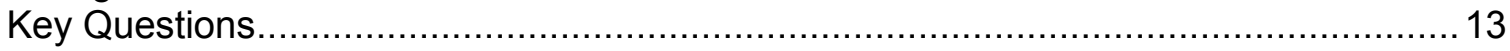

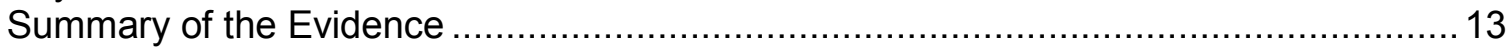

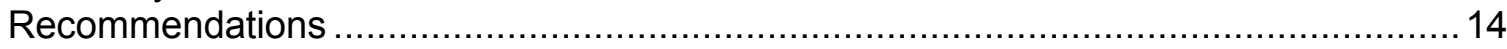

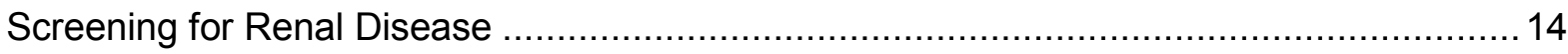

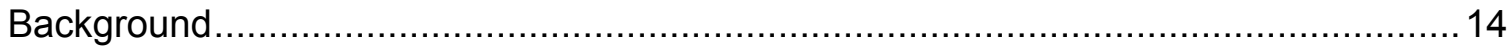

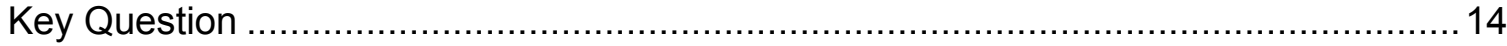

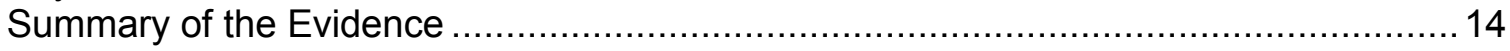

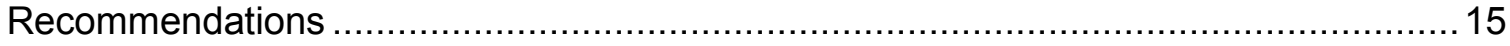

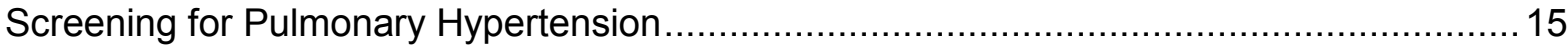

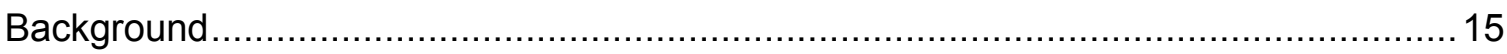

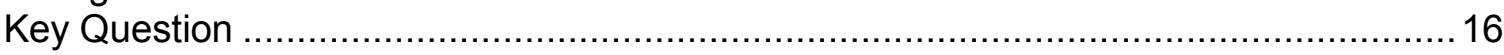

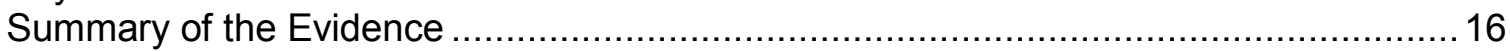

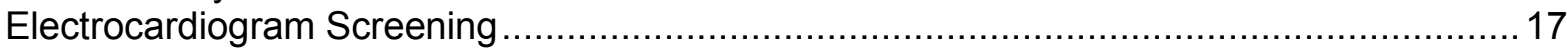

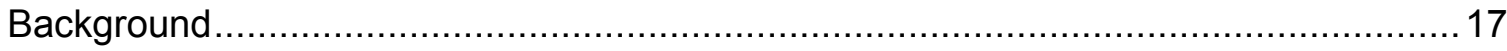

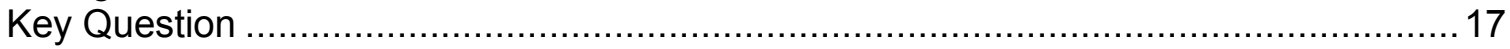

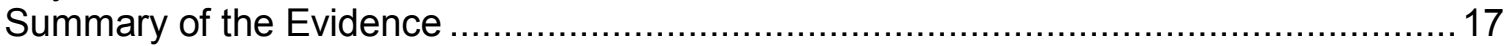

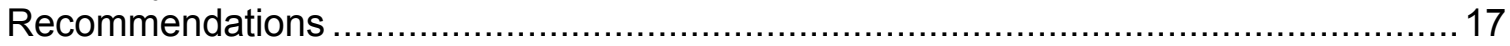

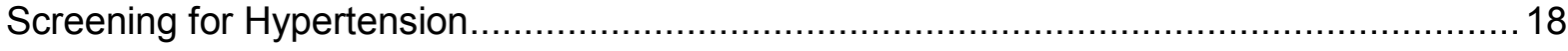

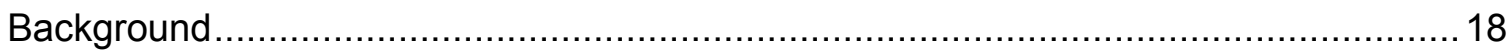

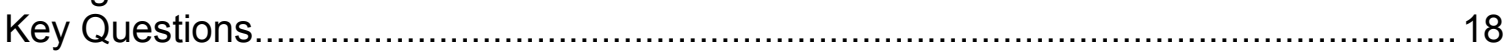

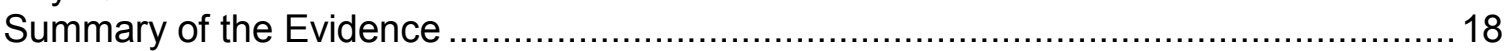

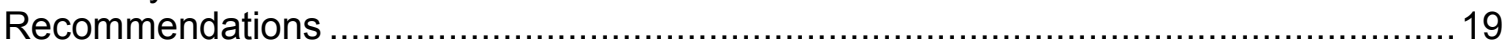

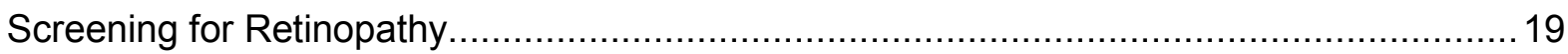

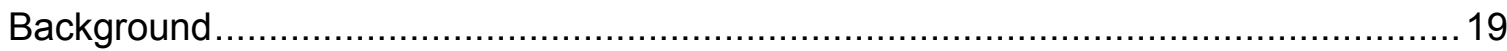

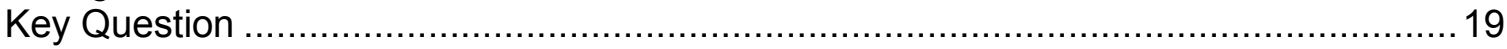

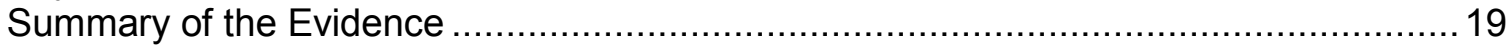

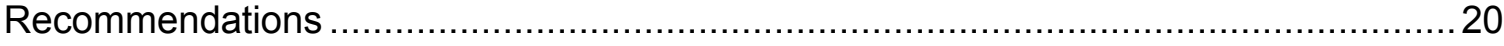




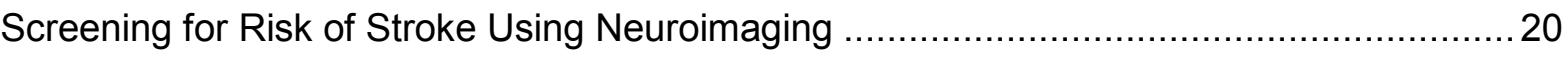

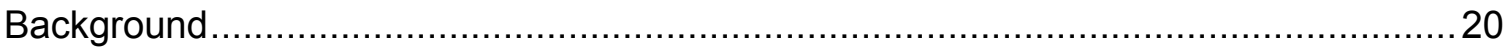

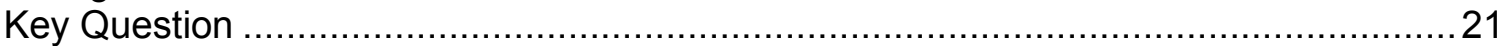

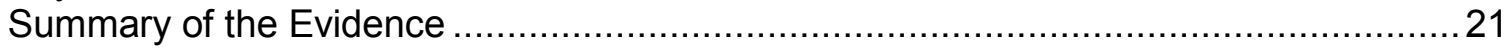

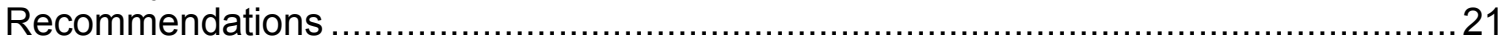

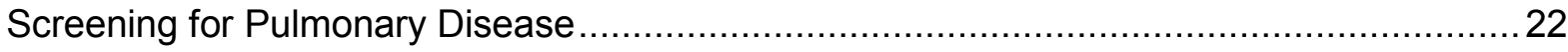

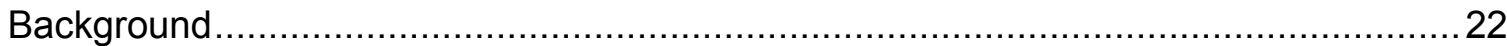

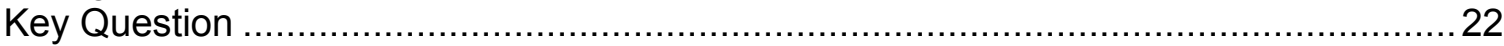

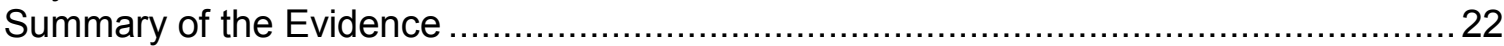

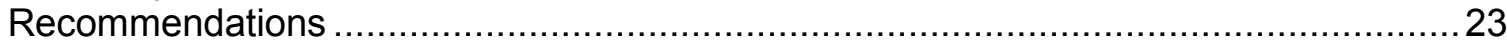

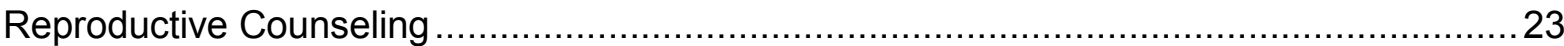

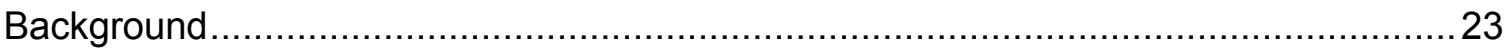

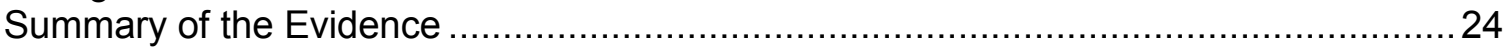

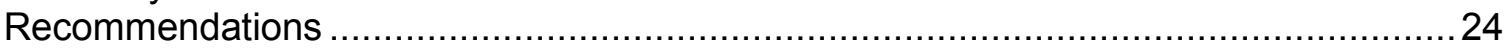

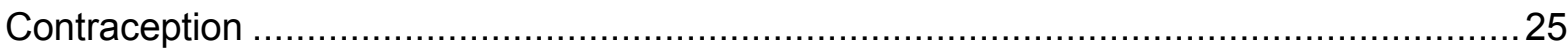

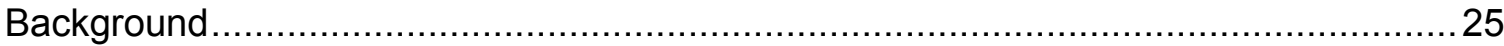

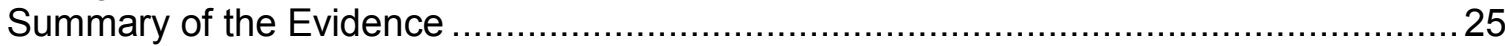

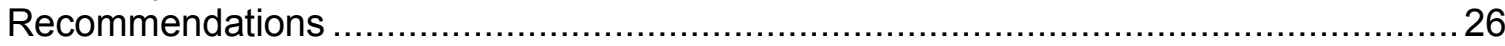

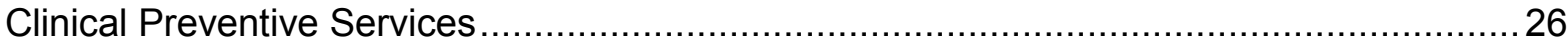

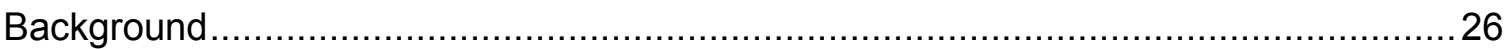

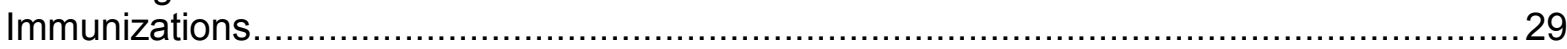

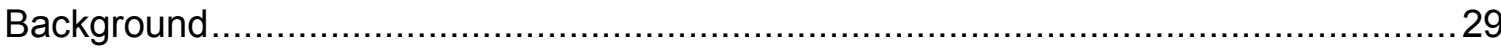

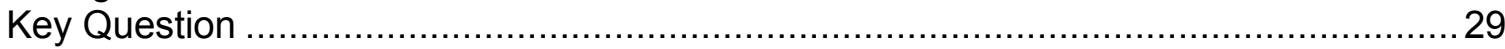

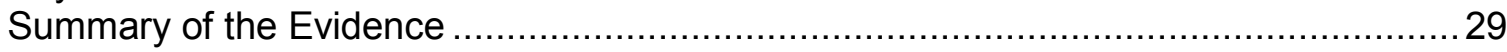

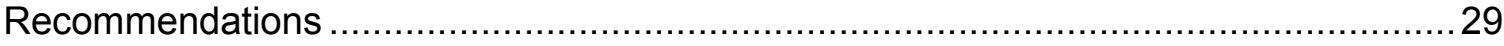

Chapter 3: Managing Acute Complications of Sickle Cell Disease .............................................. 31

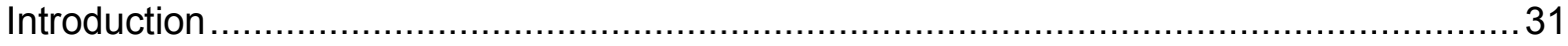

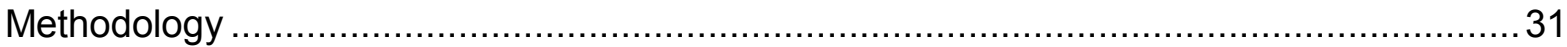

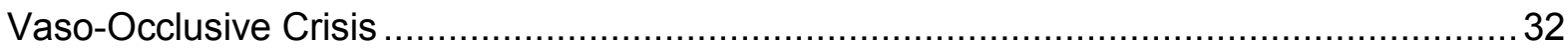

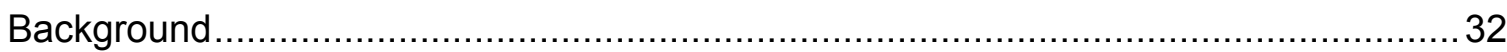

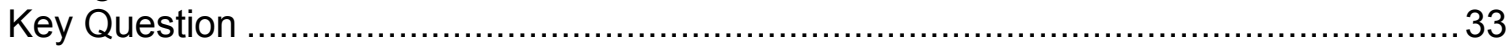

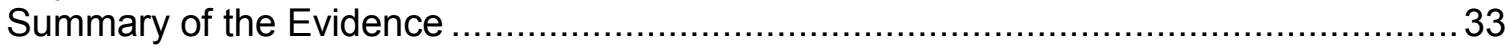

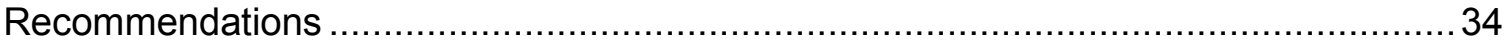

Fever

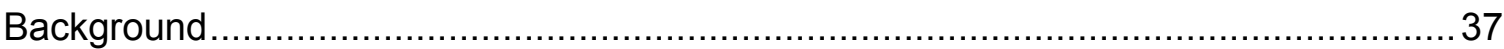

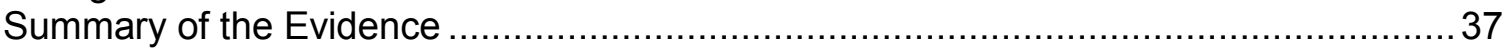

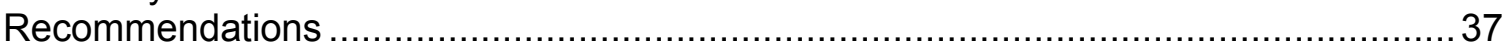

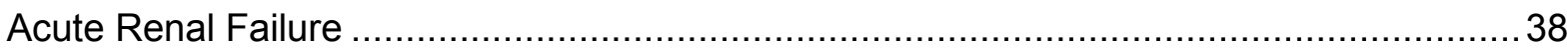

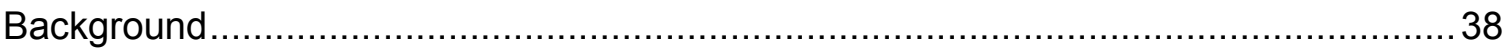

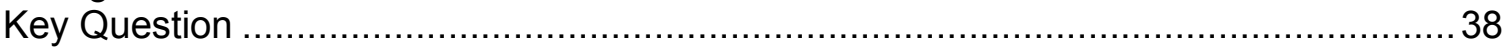

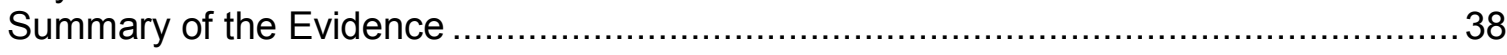

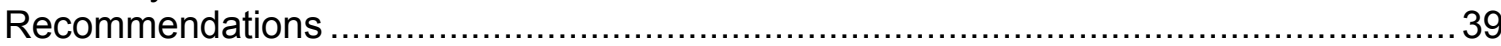

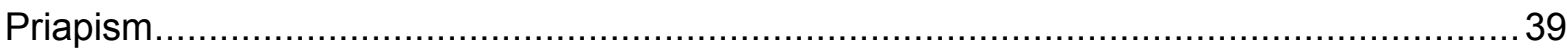

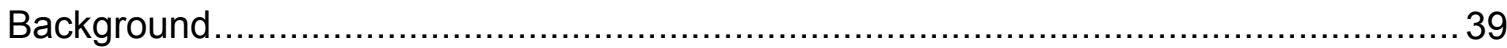

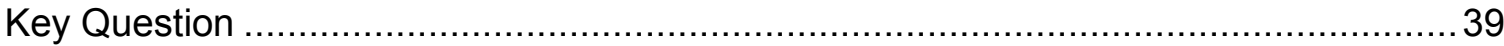

Summary of the Evidence ..................................................................................

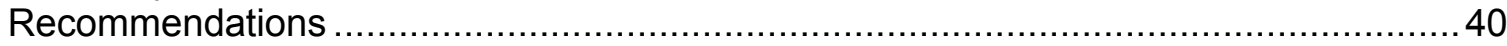

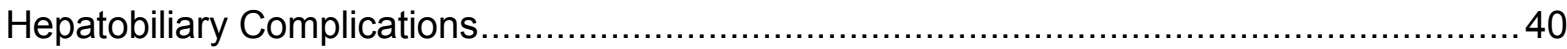

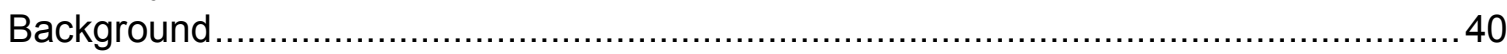

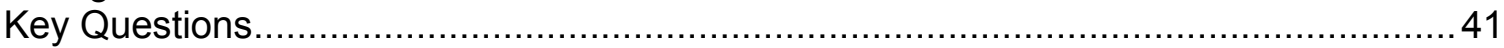

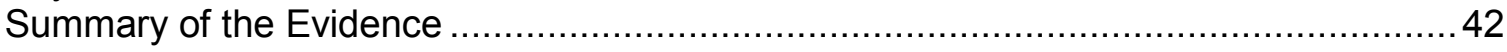

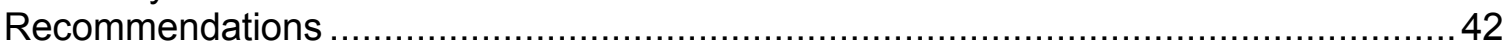




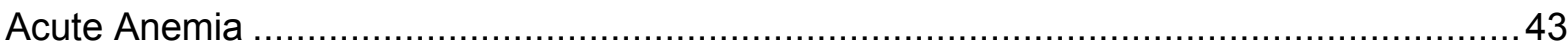

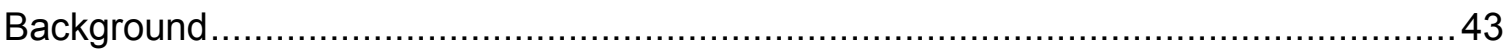

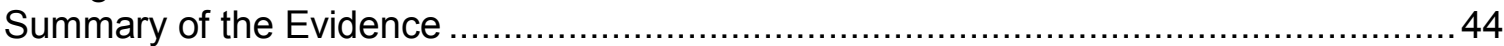

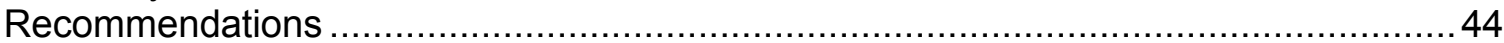

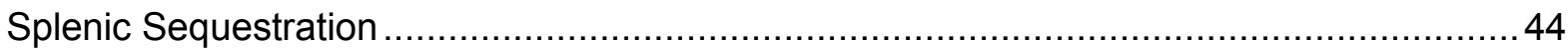

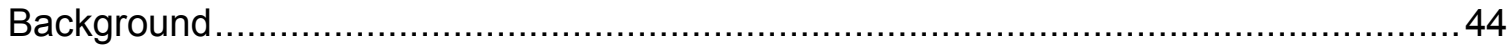

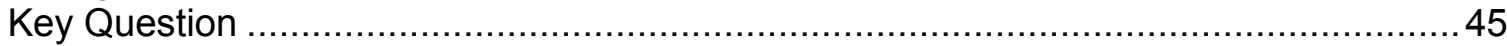

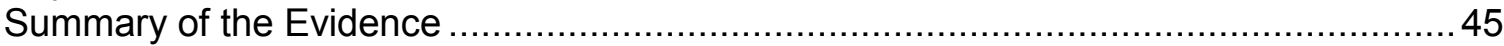

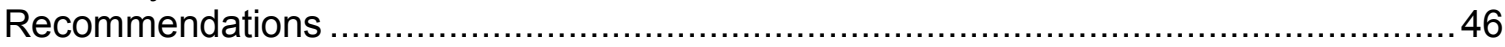

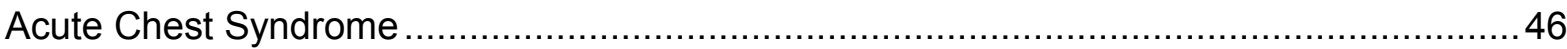

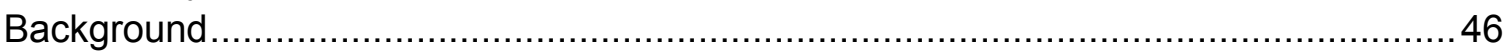

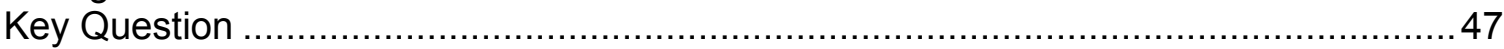

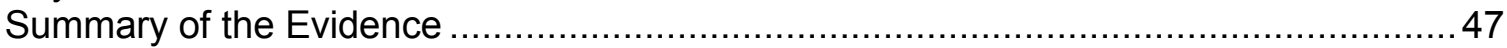

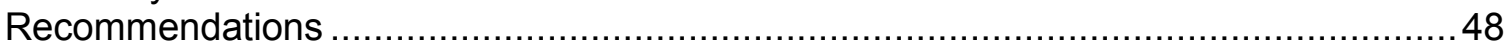

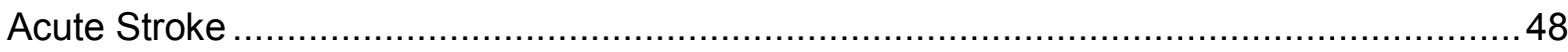

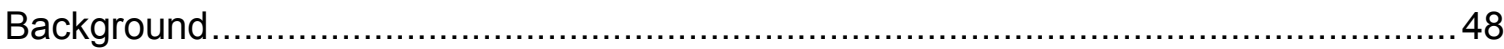

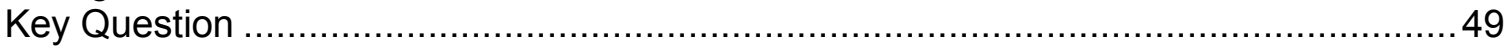

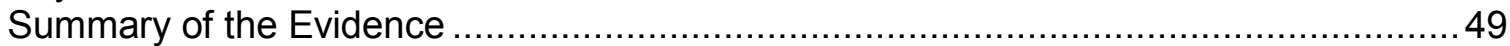

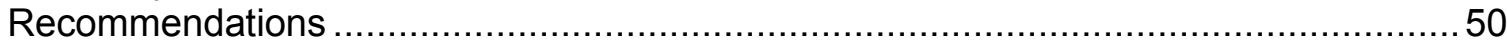

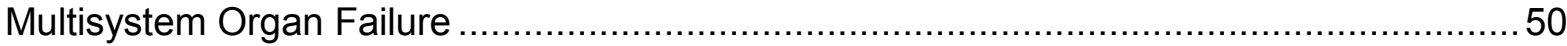

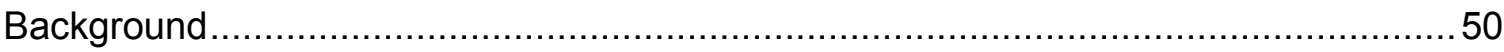

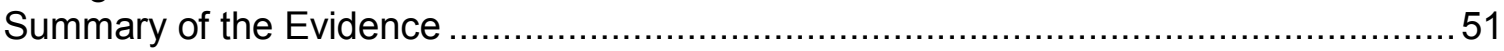

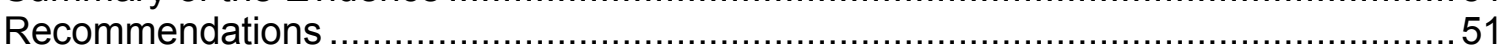

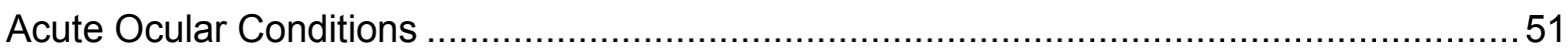

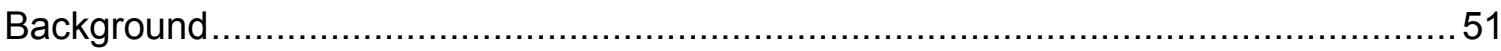

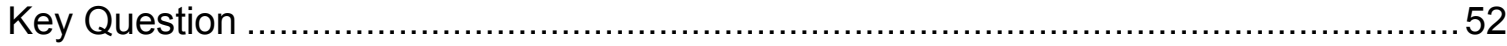

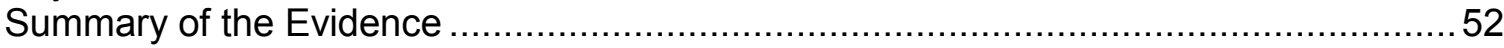

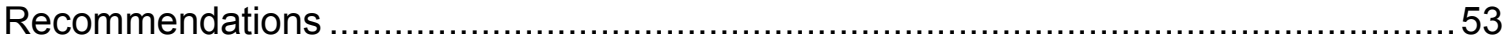

Chapter 4: Managing Chronic Complications of Sickle Cell Disease ..................................55

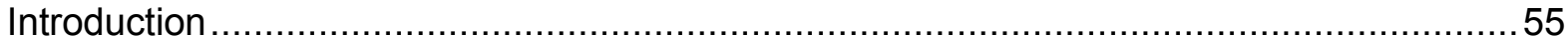

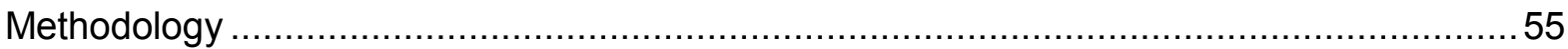

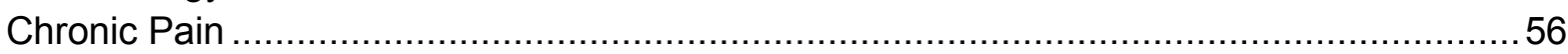

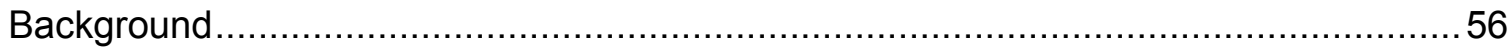

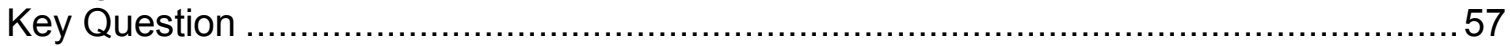

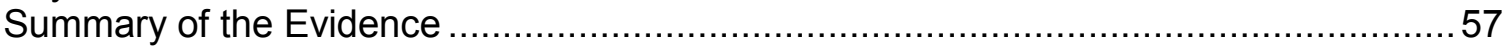

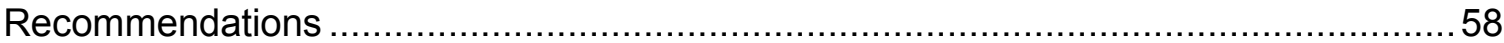

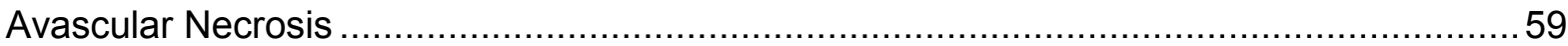

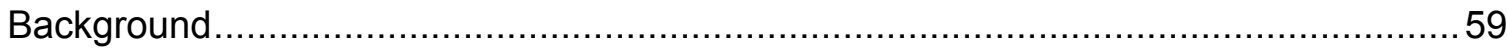

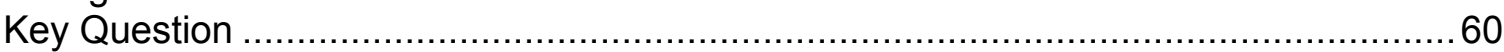

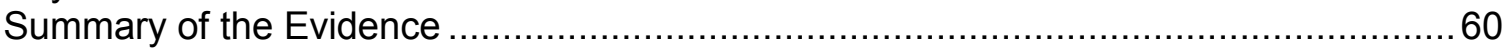

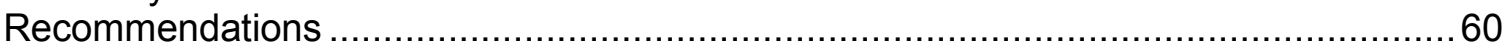

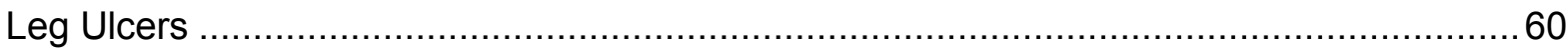

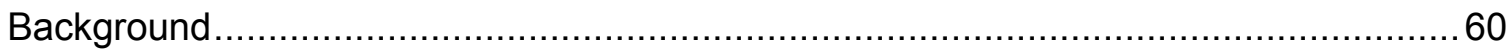

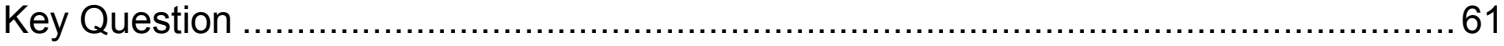

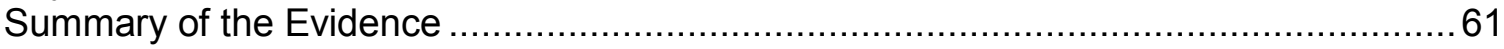

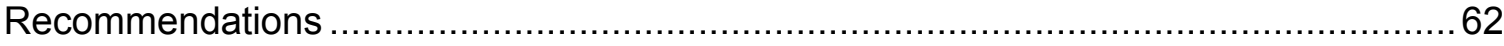

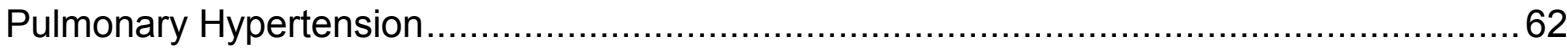

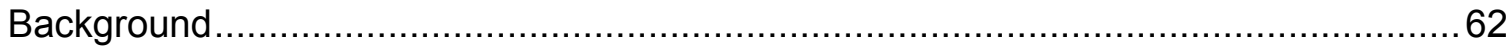

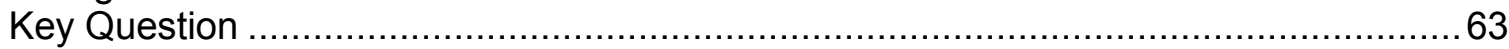

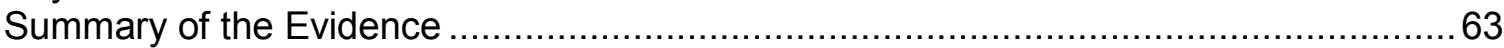

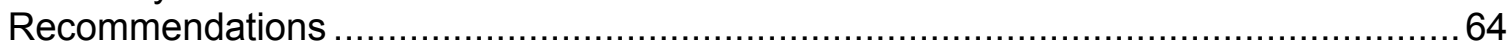




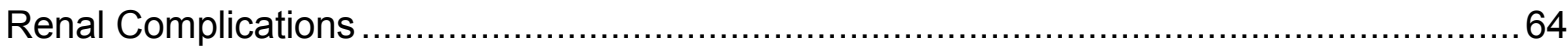

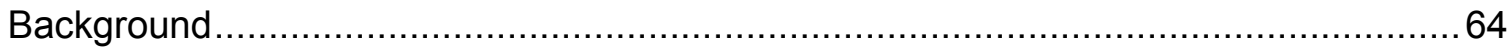

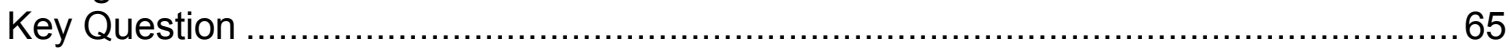

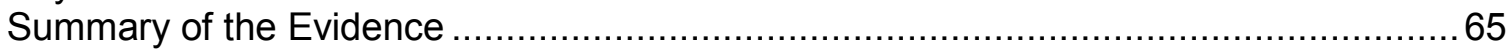

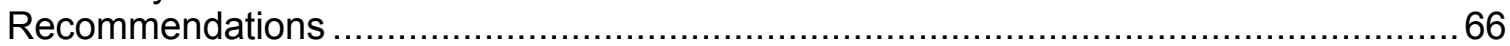

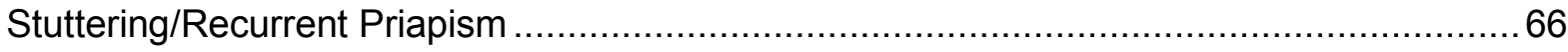

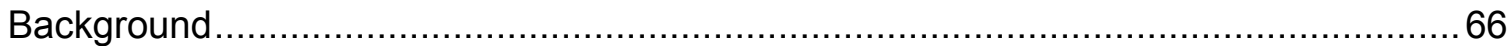

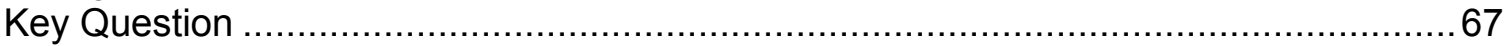

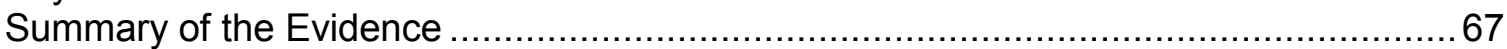

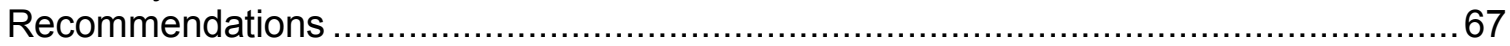

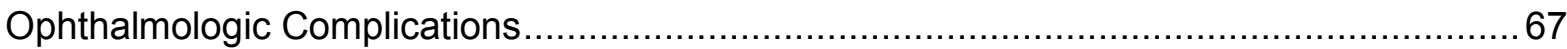

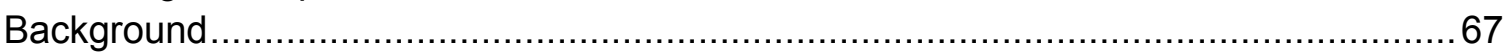

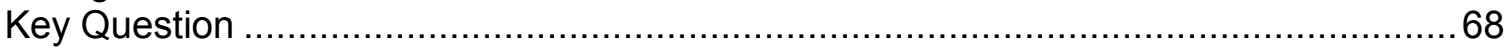

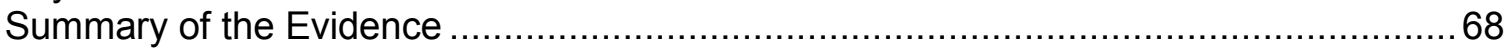

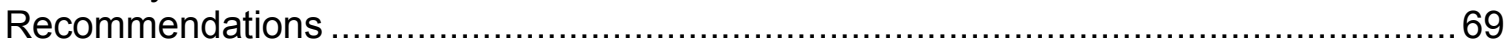

Chapter 5: Hydroxyurea Therapy in the Management of Sickle Cell Disease ……..................71

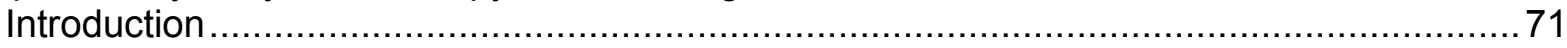

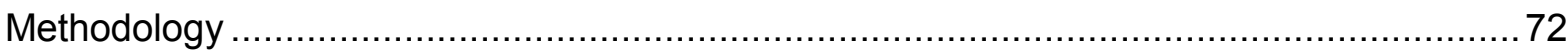

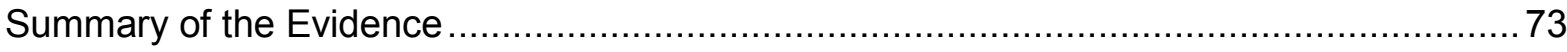

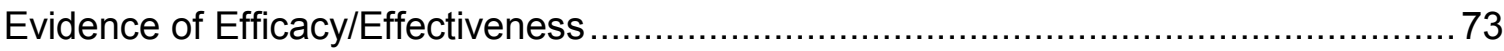

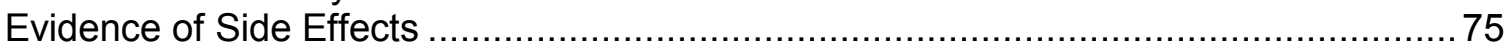

Evidence Supporting Use of a Treatment Protocol ................................................ 76

Additional Considerations............................................................................ 76

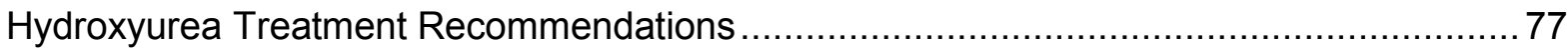

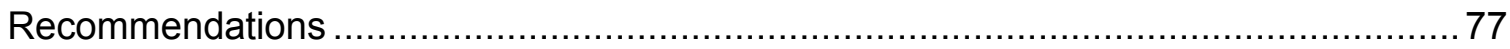

Consensus Treatment Protocol and Technical Remarks for the Implementation of

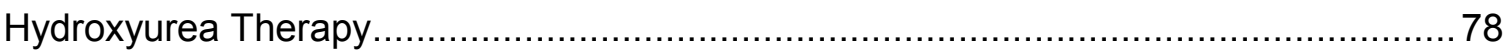

Chapter 6: Blood Transfusion in the Management of Sickle Cell Disease ...............................79

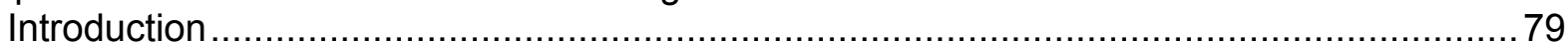

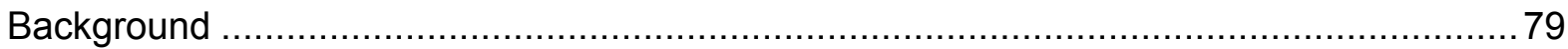

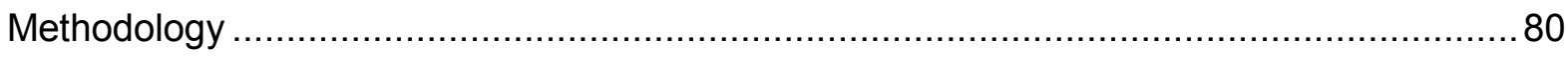

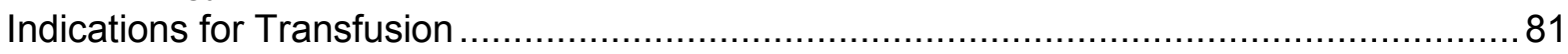

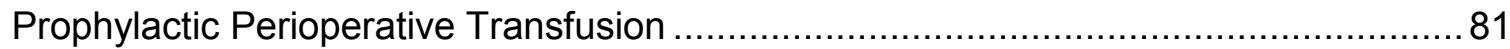

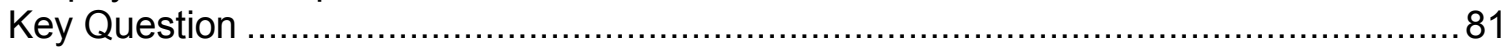

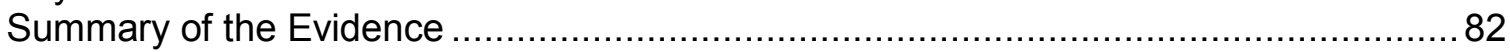

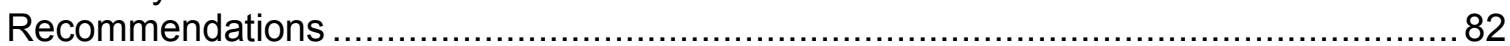

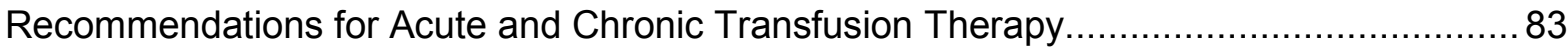

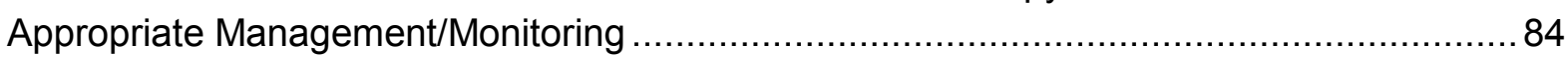

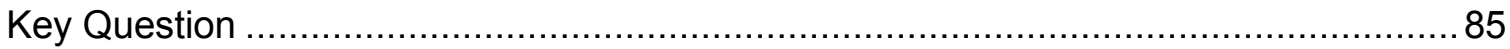

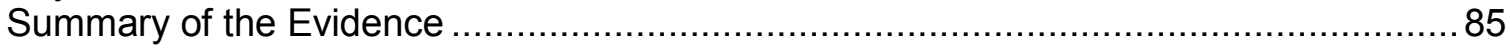

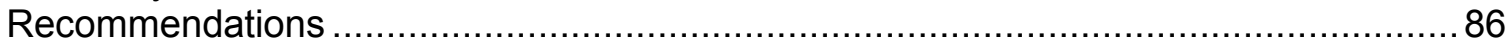

Consensus Protocol for Monitoring Individuals on Chronic Transfusion Therapy ................8 87

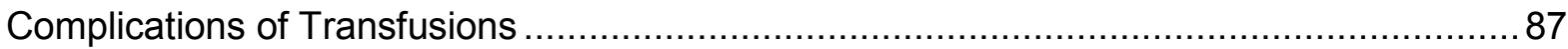

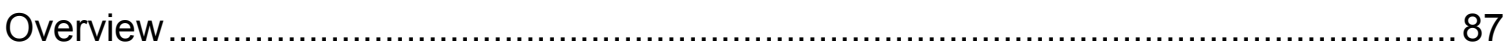

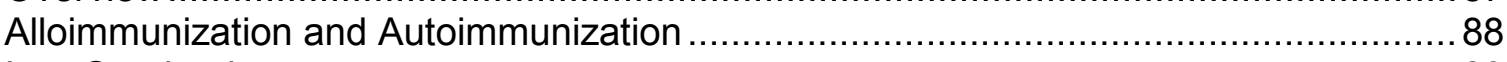

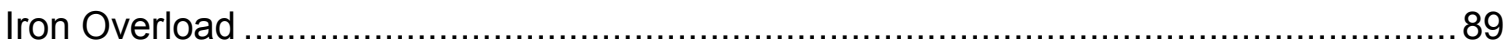

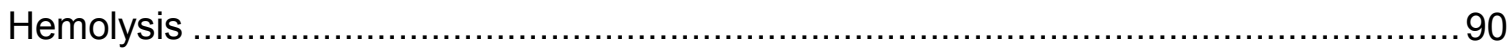

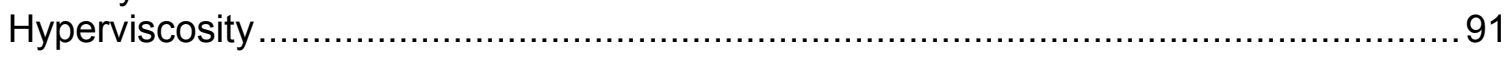

Recommendations for the Management and Prevention of Transfusion Complications .......91

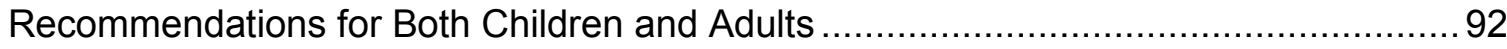


Chapter 7: Looking Forward 93

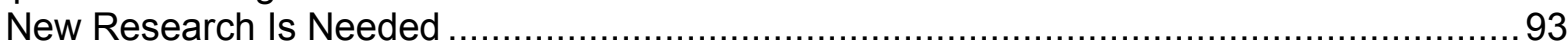

Data Systems That Meet the Highest Standards of Scientific Rigor Can Be Invaluable

Resources. .93

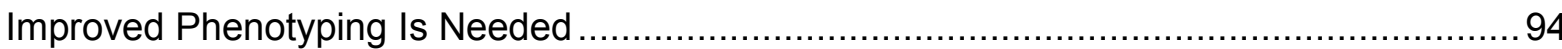

Broad Collaborations for Research and Care .......................................................... 94

Beyond Efficacy: From Bench to Bedside and the Community..................................... 94

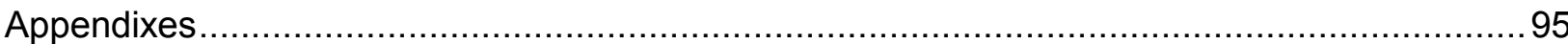

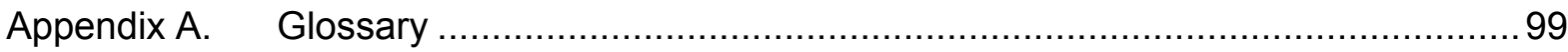

Appendix B. PICOS Questions by Chapter ..................................................... 109

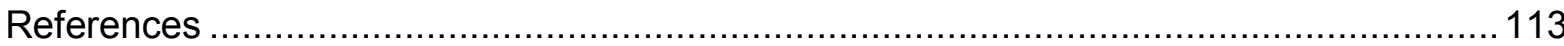

\section{List of Exhibits}

Exhibit 1a. Typical Laboratory Findings in Sickle Cell Disease ..................................... 2

Exhibit 1b. Typical Laboratory Findings in Sickle Cell Trait (Provided for Comparison)............. 2

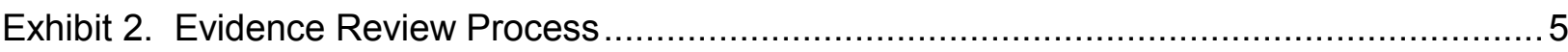

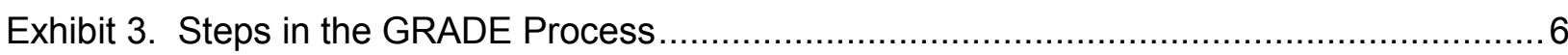

Exhibit 4. GRADE Recommendations-A Closer Look ................................................ 7

Exhibit 5. Summary of U.S. Preventive Services Task Force's General

Recommendations That Are Also Applicable to Persons With Sickle Cell Disease ............ 27

Exhibit 6. Immunization Recommendations as Adapted from the Advisory Committee

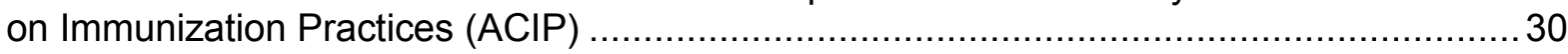

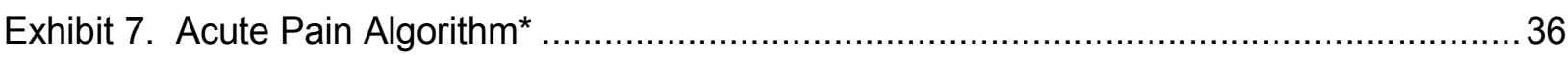

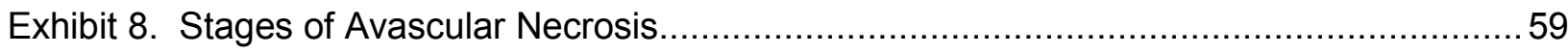

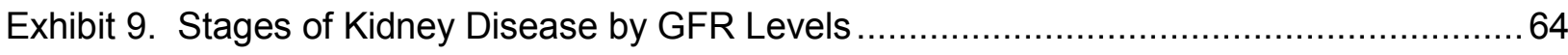

Exhibit 10. Stages of Proliferative Sickle Retinopathy (PSR) ........................................68

Exhibit 11. Participant Enrollment Criteria for Placebo-Controlled Randomized Controlled Trials of Hydroxyurea Therapy in Sickle Cell Disease ................................... 74

Exhibit 12. Evidence Profile-Evidence of Efficacy/Effectiveness for Children and Adults With Sickle Cell Anemia (Hydroxyurea Versus Usual Care) ................................ 75

Exhibit 13. Evidence Profile_Evidence of Side Effects in Sickle Cell Anemia .......................75

Exhibit 14. Acute Complications—Graded Recommendations To Transfuse .........................83

Exhibit 15. Acute Complications_Consensus Recommendations To Transfuse .................... 83

Exhibit 16. Acute Complications-Graded Recommendations When Transfusion Is

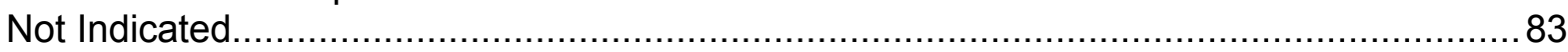

Exhibit 17. Acute Complications-Consensus Recommendations When Transfusion Is Not Indicated....

Exhibit 18. Chronic Complications-Graded Recommendations for When To Initiate a Chronic Transfusion Program .....

Exhibit 19. Chronic Complications-Graded Recommendations for When Transfusion is Not Indicated

Exhibit B-1. PICOS Approach for Health Maintenance Chapter: Antibiotics 
Exhibit B-2. PICOS Approach for Health Maintenance Chapter: Screening B-109

Exhibit B-3a. PICOS Approach for Health Maintenance Chapter: Blood Pressure (Question 1) B-110

Exhibit B-3b. PICOS Approach for Health Maintenance Chapter: Blood Pressure

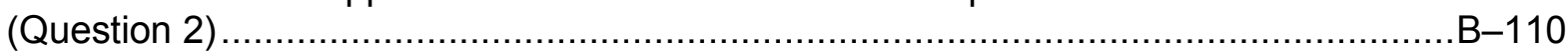

Exhibit B-4. PICOS Approach for Acute and Chronic Complications Chapters..................B-110

Exhibit B-5. PICOS Approach for Hydroxyurea Chapter......................................... -111

Exhibit B-6. PICOS Approach for Transfusion Chapter ........................................... B-111 


\section{Foreword}

The purpose of the "Evidence-Based Management of Sickle Cell Disease: Expert Panel Report (EPR), 2014" is to synthesize the available scientific evidence on sickle cell disease and offer guidance to busy primary care clinicians. Readers of this report should remember that this document is intended to provide guidance for management, not to be rigidly prescriptive. The panel recognizes that the responsible clinician's judgment regarding the management of patients remains paramount. Therefore, the Expert Panel Report is a tool to be adopted and implemented in local and individual settings, and to provide an opportunity for shared decisionmaking in which providers and patients are both fully engaged.

The EPR has been developed under the outstanding leadership of panel co-chairs Drs. George Buchanan and Barbara Yawn. The production of this report generated much discussion regarding the quality of the available scientific literature, its interpretation, and its practical application. In the end, priority was given to delivering a document that both objectively evaluated and organized the evidence and could be put into practice in the clinical setting, because effective implementation is ultimately what is needed to bring about a change in outcomes.

The NHLBI is grateful for the tremendous dedication of time and the outstanding work of the expert panel, as well as the advice of invited outside experts, the National Blood Disorders Program Coordinating Committee, and other stakeholder groups in developing this report. The invaluable comments from professional societies; voluntary health, government, consumer/patient advocacy organizations; and industry during the public review period greatly enhanced the scientific value and practical utility of this document.

By developing this landmark report, the expert panel has taken a tremendous step towards addressing the health needs of the person with sickle cell disease in the primary care setting. It is incumbent upon the team of health professionals caring for these individuals to change the landscape of care by providing this state-of-the-science care. We are excited to be joined by all concerned in efforts to reach our common ultimate goal: improved health outcomes and quality of life for every person living with sickle cell disease.

Gary H. Gibbons, M.D. Director National Heart, Lung, and Blood Institute

Susan B. Shurin, M.D. Former Deputy Director National Heart, Lung, and Blood Institute 



\section{Expert Panel}

\section{Co-Chairs}

George R. Buchanan, M.D.

University of Texas Southwestern Medical Center

Dallas, TX
Barbara P. Yawn, M.D., M.Sc., M.S.P.H.

University of Minnesota

Rochester, MN

\section{Members}

Araba N. Afenyi-Annan, M.D., M.P.H.

University of North Carolina at Chapel Hill

Chapel Hill, NC

\section{Samir K. Ballas, M.D.}

Thomas Jefferson University, Cardeza Foundation

Philadelphia, PA

Kathryn L. Hassell, M.D.

University of Colorado Denver

Aurora, $\mathrm{CO}$

Andra H. James, M.D., M.P.H.

University of Virginia

Charlottesville, VA

Lanetta Jordan, M.D., M.P.H., M.S.P.H.

Foundation for Sickle Cell Disease Research

University of Miami, Miller School of Medicine

Miami, FL

Sophie M. Lanzkron, M.D., M.H.S.

Johns Hopkins School of Medicine

Baltimore, MD

\section{Richard Lottenberg, M.D.}

University of Florida

Gainesville, FL

William J. Savage, M.D., Ph.D.

Brigham and Women's Hospital and Harvard

Medical School

Boston, MA
Paula J. Tanabe, Ph.D., R.N., F.A.E.N., F.A.A.N. Duke University, Schools of Nursing and Medicine

Durham, NC

Russell E. Ware, M.D., Ph.D.

Cincinnati Children's Hospital Medical Center

Cincinnati, $\mathrm{OH}$

Methodologist

M. Hassan Murad, M.D., M.P.H.

Mayo Clinic

Rochester, MN

National Heart, Lung, and Blood Institute Staff

Joylene John-Sowah, M.D., M.P.H. (Lead)

Jonathan Goldsmith, M.D., F.A.C.P.

Edward Donnell Ivy, M.D., M.P.H. ${ }^{a}$

Eduardo Ortiz, M.D., M.P.H. ${ }^{\mathrm{a}}$

Denise Simons-Morton, M.D., Ph.D. ${ }^{\text {a }}$

Contractor Staff

Robinson Fulwood, Ph.D., M.S.P.H., (Kelly

Government Services); Ann Horton, M.S.; Mandy

David, P.A.-C; Angela Jehle; Susan Bratten;

Stephanie Neuben; Marcia Bache, M.M.Sc., RD;

Richard Yelle ${ }^{\mathrm{b}}$; Yajie Li ${ }^{\mathrm{b}}$, M.D., M.S. (American

Institutes for Research, Rockville, MD)

\footnotetext{
${ }^{a}$ These individuals contributed to the development of these guidelines during their tenure with the NHLBI Division for the Application of Research Discoveries. ${ }^{\mathrm{b}}$ These individuals contributed to the development of these guidelines during their tenure with American Institutes for Research (AIR).
} 


\section{Financial and Other Disclosures}

NHLBI established the expert panel and invited the panel members. All members served as volunteers and received no compensation from NHLBI or any other entity for their participation.

During the development of these guidelines, measures were taken to ensure the transparency of the evidence review process and to manage all potential or perceived conflicts of interest. At the initial expert panel meeting, expert panel members were asked by the panel co-chairs to disclose interests and relationships that could potentially influence their participation or pose a potential conflict of interest. The responses are provided below.

Araba N. Afenyi-Annan, M.D., M.P.H.Consultant, Transfusion Safety Summit: Risks Associated with Iron Toxicity in Transfusional Medicine-Novartis Pharmaceuticals Corporation (November 2008); Duke University Comprehensive Sickle Cell Center, Mentored Research Training Supplement (April 2005-April 2006); Expert Witness for Hall, Booth, Smith \& Slover, P.C. (2010-present)

Samir K. Ballas, M.D.--Speaker's Bureau, Novartis; Sickle Cell Advisory Board, HemaQuest; U.S. Sickle Cell Advisory Board, Sangart

Kathryn L. Hassell, M.D.-Advisory Board, ApoPharma; Consultant, AGA Medical Corp.; Consultant and Principal Investigator of Local Site Multicenter Sickle Cell Study, Terumo, Inc.; Principal Investigator of Local Site Multi-Center Sickle Cell Study, GlycoMimetics, Inc.; Principal Investigator of Local Site Multi-Center Sickle Cell Study, Emmaus, Inc.; Board of Directors, Mount Evans Home Health \& Hospice; Medical Advisory Board, Foundation for Women and Girls with Blood Disorders; Medical Advisory Board, PFO Research Foundation

Andra H. James, M.D., M.P.H.-Consultancy for the von Willebrand Disease Medical Advisory Board for CSL Behring; Research study of antithrombin levels in pregnancy for
Grifols/Talecris; Study of von Willebrand factor levels and fibrinogen levels post partum for CSL Behring; Expert witness for Johnson \& Johnson and Sanofi-Aventis

\section{Lanetta Jordan, M.D., M.P.H., M.S.P.H.-}

National Heart, Lung, and Blood Advisory Council; Faculty Chair, Sickle Cell Disease Association of America, Inc. (SCDAA) and National Initiative for Children's Healthcare Quality (NICHQ) for Health Resources and Services Administration-funded Sickle Cell Disease Treatment Demonstration Program; AESRx Medical Advisory Council; Prolong Pharmaceutical Medical Advisory Board; Consultant for NKT Therapeutics, TriStem, Pfizer, and Novartis; Board Member, Foundation for Women and Girls with Blood Disorders and Miami YWCA

Sophie M. Lanzkron, M.D., M.H.S.-Scientific Advisory Board for HemaQuest; Principal investigator on studies sponsored by Emmaus, GlycoMimetics, Inc., and Novartis

Paula J. Tanabe, Ph.D., R.N., M.S.N., M.P.H.Partner, ESI Triage Research Team, LLC; Principal investigator on Agency for Healthcare Research and Quality research grant; Subcontractor to the Michigan Public Health Institute and the Health Resources and Services Administration (HRSA) to conduct a project in SCD, pediatrics, emergency department (ED) research; recipient of Duke School of Nursing grant to complete a project to measure the effect of a high dose opioid protocol to treat adults with a vaso-occlusive crisis (VOC) in the ED; Expert witness consultant on one SCD legal case

Russell E. Ware, M.D., Ph.D. - Consultant for Bayer, Novartis Pharmaceuticals, and Sobi

No relationships to disclose: George R. Buchanan, M.D.; Richard Lottenberg, M.D.; William J. Savage, M.D., Ph.D.; Barbara P. Yawn, M.D., M.Sc., M.S.P.H. 


\section{Expert Reviewers}

Prior to publication of the final report, the panel sought input from independent expert peer reviewers without financial conflicts of interest. The conclusions and synthesis of the scientific literature presented in this report represent a consensus but do not necessarily represent the views of individual reviewers.

The list of expert peer reviewers follows.

\section{Ofelia Alvarez, M.D.}

Professor of Clinical Pediatrics

Director, Pediatric Sickle Cell Program

Co-Director, University of Miami Sickle Cell

Center

University of Miami Miller School of Medicine

Miami, FL

\section{Rita Bellevue, M.D.}

Director, Comprehensive Sickle Cell/Thalassemia Program

New York Methodist Hospital

Assistant Professor of Medicine

Weill Cornell Medical College

New York, NY

\section{David C. Brousseau, M.D., M.S.}

Professor of Pediatrics

Director, Medical School Physician Scientist

Pathway

Medical College of Wisconsin

Chief, Section of Pediatric Emergency Medicine

Children's Hospital of Wisconsin

Milwaukee, WI

\section{Tiffiny Diers, M.D.}

Assistant Professor of Medicine

University of Cincinnati

Cincinnati, $\mathrm{OH}$
John Epling, M.D., M.S.Ed., F.A.A.F.P.

Associate Professor and Chair

Department of Family Medicine

Co-Director, Studying-Acting-Learning-Teaching

Network (SALT-Net)

Associate Professor

Public Health and Preventive Medicine

SUNY Upstate Medical University

Syracuse, NY

Julia C. Finkel, M.D.

Professor of Anesthesiology and Pediatrics

The George Washington University

Principal Investigator, The Sheikh Zayed Institute

for Pediatric Surgical Innovation

Associate Chief and Director of Research

Division of Anesthesiology and Pain Medicine

Children's National Medical Center

Washington, DC

Susan K. Jones, R.N.

Clinical Research Supervisor

UNC Comprehensive Sickle Cell Program

University of North Carolina, Chapel Hill

Chapel Hill, NC

Rebecca Kruse-Jarres, M.D., M.P.H.

Associate Professor of Clinical Medicine

Director, Adult Sickle Cell Program

Department of Medicine

Section of Hematology/Oncology

Tulane University

New Orleans, LA

Abdullah Kutlar, M.D.

Professor of Medicine

Division of Hematology/Oncology and Stem Cell

Transplantation

Director

MCG Sickle Cell Center

Georgia Regents University

Augusta, GA 


\section{Annette Lavendar}

Nurse Practitioner

Mercy Health Partners

Cincinnati, $\mathrm{OH}$

Brigitta U. Mueller, M.D., M.H.C.M.

Professor of Pediatrics

Baylor College of Medicine

Clinic Chief and Director

Division of Clinical Operations, Quality \& Safety

Texas Children's Cancer and Hematology Centers

Medical Director, Care Management

Texas Children's Hospital

Houston, TX

Linda Overholser, M.D., M.P.H.

Division of General Internal Medicine

University of Colorado Denver

Denver, CO

Gwendolyn Poles, D.O.

Faculty, IM Residency Program

PinnacleHealth System

Kline Health Center

Harrisburg, PA

Sohail Rana, M.D.

Professor

Department of Pediatric and Child Health

Howard University College of Medicine
Wally R. Smith, M.D.

Professor of Medicine

Virginia Commonwealth University

Richmond, Virginia

John Strouse, M.D.

Assistant Professor of Pediatrics and Medicine

Division of Pediatric Hematology

Department of Pediatrics

Division of Hematology

Department of Medicine

Johns Hopkins University School of Medicine

Baltimore, MD

Alexis A. Thompson, M.D., M.P.H.

Sarah and A. Watson Armour Chair in Childhood

Cancer and Blood Diseases

Hematology Section Head

Ann \& Robert H. Lurie Children's Hospital of

Chicago

Professor of Pediatrics

Feinberg School of Medicine

Northwestern University

Chicago, IL

Knox H. Todd, M.D., M.P.H.

Professor and Chair

Department of Emergency Medicine

MD Anderson Cancer Center

Houston, TX

Special thanks to the American Academy of

Pediatrics for their ongoing collaboration and review. 


\section{Expert Panel Report Endorsements}

Academy of Emergency Medicine (AAEM)

American Academy of Pediatrics (AAP)

American Academy of Physician Assistants (AAPA)

American Osteopathic Association (AOA)

American Society of Hematology (ASH)

American Society of Pediatric Hematology/Oncology (ASPHO)

International Association of Sickle Cell Nurses and Physician Assistants (IASCNAPA)

National Black Nurses Association (NBNA)

National Initiative for Children's Health Quality (NICHQ)

National Medical Association (NMA)

Sickle Cell Disease Association of America (SCDAA) 



\section{Chapter 1: Introduction and Methodology}

These guidelines were developed by an expert panel composed of health care professionals with expertise in family medicine, general internal medicine, adult and pediatric hematology, psychiatry, transfusion medicine, obstetrics and gynecology, emergency department nursing, and evidence-based medicine. Panel members were selected by the National Heart, Lung, and Blood Institute's (NHLBI's) leadership.

The purpose of these guidelines is to help people living with sickle cell disease (SCD) receive appropriate care by providing the best science-based recommendations to guide practice decisions. The target audience is primary care providers and other clinicians, nurses, and staff who provide emergency or continuity care to individuals with SCD.

NHLBI sponsored the development of these guidelines to assist health care professionals in the management of common issues, including routine health maintenance, the recognition and treatment of common acute and chronic complications and comorbidities of SCD, as well as the indications for and monitoring of hydroxyurea and blood transfusion therapy. The guidelines address the care of infants, children, adolescents, and adults with SCD, with the goal of facilitating high-quality and appropriate care for all individuals with this disease.

\section{Historical Perspective, Epidemiology, and Definitions}

SCD was first reported in the literature in November 1910 by James B. Herrick, who referred to "peculiar elongated and sickle-shaped red blood corpuscles in a case of severe anemia." We have gained substantial knowledge about SCD since that first description. Today there is hope for a cure using hematopoietic stem cell transplantation (HSCT). ${ }^{2}$ However, at present, the procedure is infrequently performed and very expensive. ${ }^{3-5}$ Additional research regarding patient and donor selection and the specific transplantation procedure is required before this potentially curative therapy will become more widely available. Two effective disease-modifying therapies for SCD—-hydroxyurea and chronic transfusion — are potentially widely available but remain underutilized..$^{6-11}$

The sickle cell mutation results in substitution of the amino acid valine for glutamic acid at the sixth position of the beta globin chain, causing formation of hemoglobin S. ${ }^{12}$ More than 2 million U.S. residents are estimated to be either heterozygous or homozygous for the genetic substitution. Most of those affected are of African ancestry or self-identify as Black; a minority are of Hispanic or southern European, Middle Eastern, or Asian Indian descent. ${ }^{13}$ It is estimated that between 70,000 and 100,000 Americans have SCD. Although SCD is associated with major morbidity, currently more than 90 percent of children with SCD in the United States and the United Kingdom survive into adulthood. ${ }^{14-16}$ However, their lifespan remains shortened by two or three decades compared to the general population. ${ }^{17,18}$

The most prevalent SCD genotypes (exhibit 1) include homozygous hemoglobin SS (HbSS) and the compound heterozygous conditions hemoglobin $\mathrm{S} \beta^{0}$-thalassemia $\left(\mathrm{HbS} \beta^{0}\right.$-thalassemia), hemoglobin $\mathrm{S} \beta^{+}$-thalassemia $\left(\mathrm{HbS} \beta^{+}\right.$-thalassemia), and hemoglobin SC disease ( $\left.\mathrm{HbSC}\right)$. $\mathrm{HbSS}$ and $\mathrm{HbS} \beta^{0}$-thalassemia are clinically very similar and therefore are commonly referred to as sickle cell anemia (SCA); these genotypes are associated with 
the most severe clinical manifestations. These guidelines are not applicable to individuals with sickle cell trait (HbAS), the carrier state.

Exhibit 1a. Typical Laboratory Findings in Sickle Cell Disease

\begin{tabular}{|l|l|l|l|l|l|l|}
\multicolumn{1}{c}{ Genotype } & \multicolumn{1}{c}{$\mathrm{Hb}^{*}(\mathrm{~g} / \mathrm{dL})^{\dagger}$} & \multicolumn{1}{c}{$\mathrm{HbS}(\%)$} & \multicolumn{1}{c}{$\mathrm{HbA}(\%)$} & \multicolumn{1}{c}{$\mathrm{HbA} 2(\%)$} & \multicolumn{1}{c|}{$\mathrm{HbF}(\%)$} & \multicolumn{1}{c|}{$\mathrm{HbC}(\%)$} \\
\hline $\mathrm{SS}$ & $6-9$ & $>90$ & 0 & $<3.5$ & $<10$ & 0 \\
\hline $\mathrm{S} \beta^{0}$-thalassemia & $7-9$ & $>80$ & 0 & $>3.5$ & $<20$ & 0 \\
\hline $\mathrm{S} \beta^{+}$-thalassemia & $9-12$ & $>60$ & $10-30$ & $>3.5$ & $<20$ & 0 \\
\hline $\mathrm{SC}$ & $9-14$ & 50 & 0 & $<3.5$ & $\leq 1.0$ & 45 \\
\hline
\end{tabular}

* Definitions for abbreviations are as follows: $\mathrm{Hb}=$ hemoglobin; $\mathrm{HbS}=$ sickle hemoglobin; $\mathrm{HbA}=$ normal adult hemoglobin; $\mathrm{HbA}_{2}=$ minor variant of adult hemoglobin; $\mathrm{HbF}=$ fetal hemoglobin; $\mathrm{HbC}=$ hemoglobin variant that causes manifestations of SCD when paired with $\mathrm{HbS}$

${ }^{\dagger}$ The hemoglobin values in this exhibit apply in the absence of a blood transfusion in the last 4 months, are not absolute, and are applicable to adults and children only (not newborns).

Exhibit 1b. Typical Laboratory Findings in Sickle Cell Trait (Provided for Comparison)

\begin{tabular}{|l|l|l|l|l|l|l|}
\hline Genotype & \multicolumn{1}{c}{$\mathrm{Hb}^{*}(\mathrm{~g} / \mathrm{dL})^{\dagger}$} & \multicolumn{1}{c}{$\mathrm{HbS}(\%)$} & $\mathrm{HbA}(\%)$ & \multicolumn{1}{c}{$\mathrm{HbA2}(\%)$} & $\mathrm{HbF}(\%)$ & $\mathrm{HbC}(\%)$ \\
\hline AS & normal & $\leq 40$ & $>60$ & $<3.5$ & $\leq 1.0$ & 0 \\
\hline
\end{tabular}

* Definitions for abbreviations are as follows: $\mathrm{Hb}=$ hemoglobin; $\mathrm{HbS}=$ sickle hemoglobin; $\mathrm{HbA}=$ normal adult hemoglobin; $\mathrm{HbA}_{2}=$ minor variant of adult hemoglobin; $\mathrm{HbF}=$ fetal hemoglobin; $\mathrm{HbC}=$ hemoglobin variant that causes manifestations of SCD when paired with $\mathrm{HbS}$

${ }^{\dagger}$ The hemoglobin values in this exhibit apply in the absence of a blood transfusion in the last 4 months, are not absolute, and are applicable to adults and children only (not newborns).

Currently in the United States, there are no comprehensive, systematically reviewed, evidence-based guidelines to assist health care professionals in the management of individuals with SCD. Providing care to these individuals can be challenging. As a result of the condition's relative rarity, there are few health care professionals who are prepared to deliver continuity care or expert consultation for patients with serious acute or chronic SCD complications. These guidelines are therefore being made available to help provide the latest evidence-based recommendations to manage this condition and to help engage health care professionals in supporting their implementation at the practice level.

\section{Overview of the SCD Guidelines Chapters}

This report begins with a chapter on comprehensive health maintenance. Many children and adults with chronic diseases such as SCD do not receive the recommended preventive care provided to other children and adults. Therefore, the guidelines summarize recommendations for health maintenance screening, testing, and immunizations as they apply to infants, children, adolescents, and adults with SCD. Generally speaking, recommendations for screening to facilitate primary and secondary prevention (e.g., asking a teen about smoking behavior or an adult woman about mammography, respectively) are often confused with recommendations for evaluating early symptoms of a disease or condition. For this and most other documents such as the recommendations of the U.S. Preventive Services Task Force (USPSTF), screening is considered testing or evaluation for a relatively common condition for which there is effective therapy prior to symptom recognition or during an asymptomatic phase. ${ }^{19}$ Generalized or universal screening is not recommended when existing therapies have not been shown to improve patient outcomes when implemented in this early 
presymptomatic phase. Information on screening and preventive care is important for all clinicians who work with individuals with SCD, including specialists who may serve as the continuity health care source for them.

Acute complications are common at all ages in individuals with SCD and are addressed in the chapter, "Managing Acute Complications of Sickle Cell Disease." Recurrent acute pain crises (also known as vasoocclusive crises) are the most common manifestation of SCD. These crises occur, usually without warning, when obstructed blood flow results in ischemic tissue injury and pain. The vascular occlusion, generally at the level of capillaries and post-capillary venules, results not only from an accumulation of adherent and sickled erythrocytes, but also from alterations involving the vascular endothelium and adhesive proteins in the plasma and on white blood cells and platelets. The management of acute pain is central to the care of individuals with SCD, yet pain is often poorly or inadequately addressed in all types of health care settings.

These guidelines include recommendations for rapid and effective pain management in people with SCD who present with such pain crises. Other significant acute complications addressed in this chapter include acute chest syndrome (ACS), stroke, splenic sequestration, acute renal failure, and cholecystitis. Neuropsychological, educational, and vocational impairment as well as common mental health issues such as depression and anxiety, which often accompany chronic illness, were considered beyond the scope of this guideline work.

Chronic complications of SCD may occur as a result of acute episodes or as chronic or recurrent events. Several of the most common of the chronic complications - including chronic pain, cholelithiasis, renal dysfunction, pulmonary hypertension, and retinal problems - are addressed in the fourth chapter, "Managing Chronic Complications of Sickle Cell Disease."

Each of the two major therapies used in individuals with SCD - hydroxyurea and chronic blood transfusionsare described in separate chapters (see "Hydroxyurea Therapy in the Management of Sickle Cell Disease," and "Blood Transfusion in the Management of Sickle Cell Disease"). These are the only currently proven diseasemodifying treatments for people with SCD. Both therapies are used in primary and secondary stroke prevention. Although neither has been shown to prevent all SCD-related organ damage, these treatment modalities can improve the quality of life for individuals with SCD. Treatment with hydroxyurea is underutilized for many people with SCA who could benefit from it. Blood transfusion therapy has at times been underutilized, overutilized, or prescribed inappropriately for both acute and chronic complications. These two chapters provide guidance regarding the appropriate use of these therapies for SCD.

\section{Process and Methodology}

The expert panel first convened in the spring of 2009 to establish the vision and purpose of the panel, discuss the process and schedule for producing the guidelines, and determine the critical areas to be addressed. Prior to this meeting, the expert panel participated in a conference call to introduce the panel's work and discuss the overarching questions that should be answered by the guidelines. Before beginning the writing of the guidelines report, the expert panel divided its work into sections dealing with preventive care or health maintenance, recognition and management of acute SCD-related complications, recognition and management of chronic SCD-related complications, and the two most broadly assessed and available disease-modifying therapies for SCD, hydroxyurea and chronic blood transfusions.

With the assistance of the methodology team and the supporting evidence center, the panel then developed key questions and literature search terms to identify evidence. The field of SCD has a limited number of randomized controlled trials (RCTs) or large prospective cohort studies to guide clinical decisionmaking; therefore, few of the recommendations in this document are based on this highest quality evidence. For 
common health issues, the panel included the evidence-based recommendations of the USPSTF ${ }^{20}$ as well as vetted recommendations of other groups. These recommendations include the SCD reproductive-related recommendations of the World Health Organization (WHO), ${ }^{21}$ the immunization recommendations of the Advisory Committee on Immunization Practices (ACIP), ${ }^{22}$ and the acute and chronic pain management recommendations of the American Pain Society (APS). ${ }^{23,24}$ These recommendations are denoted as "Consensus-Adapted."

Recognizing the need to provide practical guidance for common problems that may lie outside of the panel's evidence reviews or available science, in many areas the published evidence was supplemented by the expertise of the panel members, who have many years of experience in managing and studying individuals with SCD. Recommendations based on the opinions of the expert panel members are labeled as "Consensus-Panel Expertise." Each is clearly labeled with the strength of the recommendation and the quality of evidence available to support it.

\section{Evidence Review and Synthesis}

Beginning in April 2010, the expert panel collaborated with an independent evidence synthesis group (hereafter referred to as the methodology team) that included methodologists, librarians, and research staff with expertise in conducting systematic reviews and meta-analyses and appraising and summarizing evidence for the purpose of guideline development. The methodology team used the overarching questions, the key questions, and a list of specific guideline topics to draft an initial list of $\operatorname{PICOS}^{\mathrm{c}}$-formatted critical questions for literature searches and formal evidence appraisal and vetting (see appendix B). The methodology team developed search strategies and then conducted literature searches and prepared the evidence tables and a summary of the body of the evidence (see http://www.nhlbi.nih.gov/guidelines/scd/index.htm).

Exhibit 2 outlines the overall process for the evidence search, evidence synthesis, and recommendation development.

${ }^{\mathrm{c}}$ PICOS is a framework for developing a structured research question. It includes the following components in the statement of the critical question: $\mathrm{P}=$ Population; $\mathrm{I}=$ Intervention, exposure; $\mathrm{C}=$ Comparator; $\mathrm{O}=$ Outcome; $\mathrm{S}=$ Setting. 


\section{Exhibit 2. Evidence Review Process}

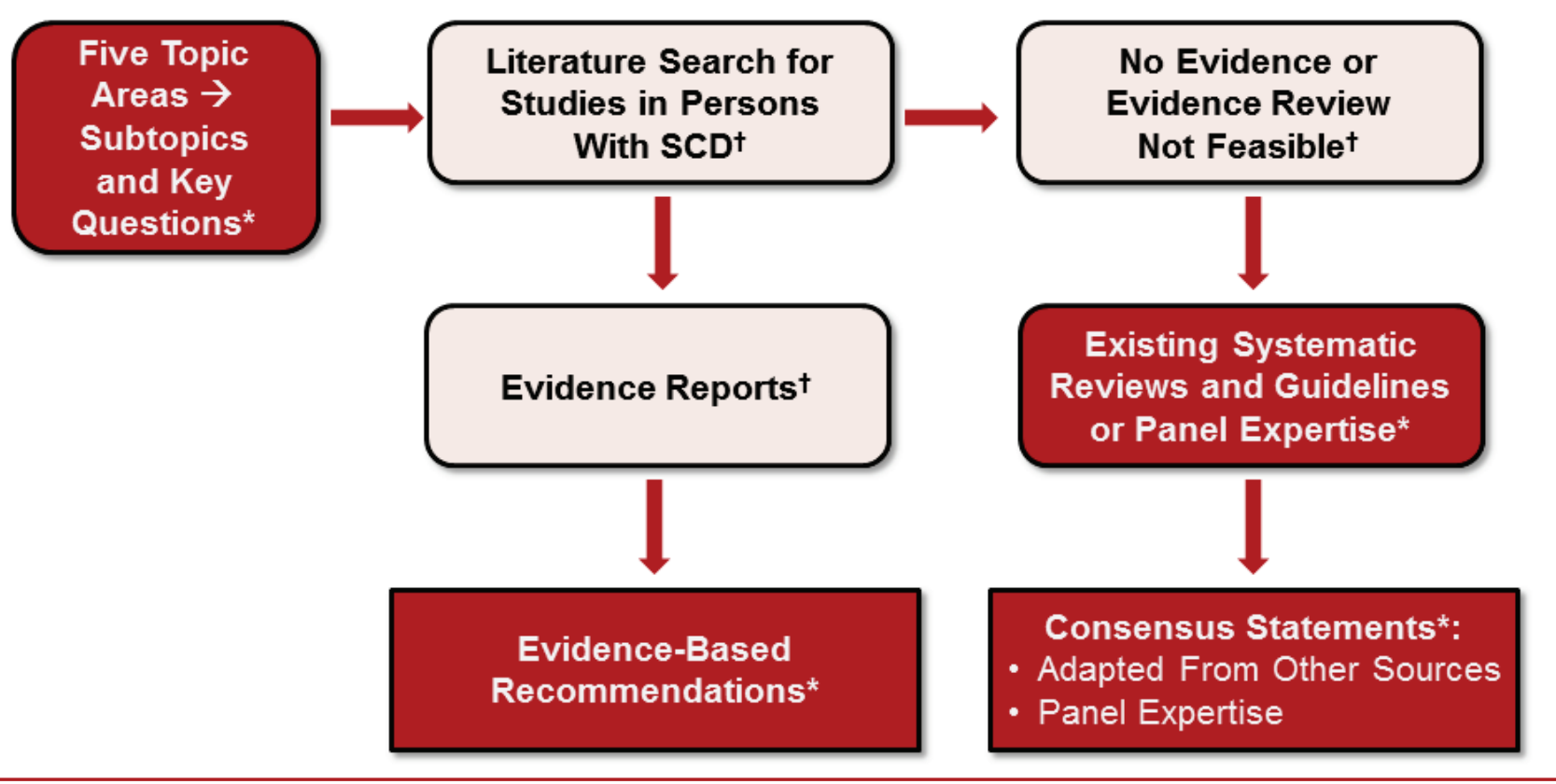

Note: Exhibit 2 shows the evidence review process. Boxes marked with an * symbol represent work conducted by the expert panel; boxes marked with $\mathrm{a}^{\dagger}$ symbol represent work conducted by the methodology team.

\section{Literature Search}

Due to the comprehensive scope of the guidelines, the search strategies for the systematic reviews were designed to have high sensitivity and low specificity; hence, the strategies were often derived from population and condition terms (e.g., people with SCD who have priapism) and not restricted or combined with outcome or intervention terms. To be inclusive of the available literature in the field, searches included randomized trials, nonrandomized intervention studies, and observational studies. Case reports and small case series were included only when outcomes involved harm (e.g., the adverse effects of hydroxyurea) or when rare complications were expected to be reported.

Literature searches involved multiple databases (e.g., Medline ${ }^{\circledR}$ In-Process \& Other Non-Indexed Citations, MEDLINE $^{\circledR}$, Embase, Cochrane Database of Systematic Reviews, Cochrane Central Register of Controlled Trials, Cumulative Index to Nursing and Allied Health Literature (CINAHL ${ }^{\circledR}$ ), TOXLINE ${ }^{\circledR}$, and Scopus) and used controlled vocabulary (prespecified) terms supplemented with keywords to define concept areas.

\section{Evidence Synthesis}

The initial literature searches performed to support these guidelines yielded 12,532 references. The expert panel also identified an additional 1,231 potentially relevant references. An updated search of randomized controlled trials (RCTs) added eight trials. ${ }^{d}$ All abstracts were reviewed independently by two reviewers using an online reference management system (DistillerSR — $\underline{\mathrm{http}}$ ://systematic-review.net) until reviewers reached adequate agreement (kappa $\geq 0.90$ ). A total of 1,575 original studies were included in the evidence tables.

Methodologists developed evidence tables to summarize individual study findings and present the quality of

${ }^{\mathrm{d}}$ An updated search was performed to span the time from June 1, 2010 through July 11, 2014. Eight additional RCTs were identified, and a supplemental table reflecting these additions was added to the evidence table document. 
evidence (i.e., confidence in the estimates of effect). The tables included descriptions of study population, SCD genotypes, interventions, and outcomes. Additional methodological details are discussed in each evidence table, including the search question, search strategy, study selection process, and list of excluded studies (see http://www.nhlbi.nih.gov/guidelines/scd/index.htm).

\section{Evidence Framework}

The methodology team used the Grading of Recommendations Assessment, Development and Evaluation (GRADE) framework ${ }^{25}$ to grade the quality of evidence, and, in concert with the panel, determine the strength of recommendations. The GRADE framework is accepted by more than 75 national and international organizations (see exhibit 3). It provides the advantages of: (a) separately judging the quality of supporting evidence and strength of recommendations, and (b) incorporating factors other than evidence in decisionmaking (e.g., the balance of benefits and harms; the perceived values and preferences of those with SCD; resources; and clinical and social context). GRADE emphasizes the use of patient-important outcomes (i.e., outcomes that affect the way patients feel, function, or survive $)^{26}$ over laboratory and physiologic outcomes.

\section{Exhibit 3. Steps in the GRADE Process}

1. Quality of evidence for each patient-important outcome is rated individually and then across outcomes

2. Randomized trials start as high quality and observational studies start as low quality

3. Quality of evidence is rated down for increased risk of

a. Risk of bias

b. Publication bias

c. Imprecision

d. Indirectness

e. Inconsistency

4. Quality of evidence is rated up for

a. Large effect

b. Dose response effect

c. When plausible confounding increases the association

5. Consider balance of benefits and harms, resources and patient's values and preferences in addition to quality of the body of evidence to determine strength of recommendations

6. The strength of recommendation is either strong or weak*

Source: Balshem H, Helfand M, Schünemann HJ, Oxman AD, Kunz R, Brozek J, Vist GE, Falck-Ytter Y, Meerpohl J, Norris S, Guyatt GH. GRADE guidelines: 3. Rating the quality of evidence. J Clin Epidemiol. 2011 Apr;64(4):401-6. ${ }^{27}$

* In these guidelines, an intermediate category of recommendation (moderate) was also used.

\section{Determining Evidence Quality}

In the GRADE framework, the quality of evidence (in this case, the body of evidence) is rated as high, moderate, low, or very low. ${ }^{28}$ The quality of evidence derived from randomized trials starts as "high," and the quality of evidence derived from observational studies starts as "low." The quality of evidence can then be lowered due to methodological limitations in individual studies (risk of bias), inconsistency across studies (heterogeneity), indirectness (the extent to which the evidence fails to apply to the specific clinical question in terms of the patients, interventions, or outcomes), imprecision (typically due to a small number of events or wide confidence intervals), and the presence of publication and reporting biases. Conversely, the quality of evidence can be upgraded in certain situations such as when the treatment effect is large or a dose-response relationship is evident. 


\section{Determining the Strength of Recommendations}

The GRADE framework rates the strength of recommendations as "strong" or "weak." However, the panel modified the GRADE system and used a third category — moderate - when they determined that patients would be better off if they followed a recommendation, despite there being some level of uncertainty about the magnitude of benefit of the intervention or the relative net benefit of alternative courses of action. The panel intends for these moderate-strength recommendations to be used to populate protocols of care and provide a guideline based on the best available evidence. The panel does not intend for weak- or moderate-strength recommendations to generate quality-of-care indicators or accountability measures or affect insurance reimbursement. Variation in care in the areas of weak- or moderate-strength recommendations may be acceptable, particularly in ways that reflect patient values and preferences. Conversely, strong recommendations represent areas in which there is confidence in the evidence supporting net benefit, and the recommendations likely apply to most individuals with SCA. For more information, see exhibit 4.

\section{Exhibit 4. GRADE Recommendations-A Closer Look}

\begin{tabular}{|c|c|c|c|}
\hline $\begin{array}{c}\text { Grade of } \\
\text { Recommendation }\end{array}$ & $\begin{array}{l}\text { Clarity of Risk } \\
\text { Benefit }\end{array}$ & $\begin{array}{c}\text { Quality of Supporting } \\
\text { Evidence }\end{array}$ & Implications \\
\hline $\begin{array}{l}\text { Strong } \\
\text { recommendation } \\
\text { High-quality evidence }\end{array}$ & $\begin{array}{l}\text { Benefits clearly outweigh } \\
\text { harms and burdens, or } \\
\text { vice versa }\end{array}$ & $\begin{array}{l}\text { Consistent evidence from } \\
\text { well-performed RCTs or } \\
\text { exceptionally strong evidence } \\
\text { from unbiased observational } \\
\text { studies* }^{*}\end{array}$ & $\begin{array}{l}\text { Recommendation can apply to most } \\
\text { patients in most circumstances. } \\
\text { Further research is very unlikely to } \\
\text { change our confidence in the estimate } \\
\text { of effect. }\end{array}$ \\
\hline $\begin{array}{l}\text { Strong } \\
\text { recommendation } \\
\text { Moderate-quality } \\
\text { evidence }\end{array}$ & $\begin{array}{l}\text { Benefits clearly outweigh } \\
\text { harms and burdens, or } \\
\text { vice versa }\end{array}$ & $\begin{array}{l}\text { Evidence from RCTs with } \\
\text { important limitations } \\
\text { (inconsistent results, } \\
\text { methodological flaws, indirect } \\
\text { or imprecise evidence), or } \\
\text { unusually strong evidence } \\
\text { from unbiased observational } \\
\text { studies }\end{array}$ & $\begin{array}{l}\text { Recommendation can apply to most } \\
\text { patients in most circumstances. } \\
\text { Further research (if performed) is } \\
\text { likely to have an impact on our } \\
\text { confidence in the estimate of effect } \\
\text { and may change the estimate. }\end{array}$ \\
\hline $\begin{array}{l}\text { Strong } \\
\text { recommendation } \\
\text { Low-quality evidence }\end{array}$ & $\begin{array}{l}\text { Benefits clearly outweigh } \\
\text { harms and burdens, or } \\
\text { vice versa }\end{array}$ & $\begin{array}{l}\text { Evidence for at least one } \\
\text { critical outcome from } \\
\text { observational studies, from } \\
\text { RCTs with serious flaws, or } \\
\text { indirect evidence }\end{array}$ & $\begin{array}{l}\text { Recommendation may change when } \\
\text { higher quality evidence becomes } \\
\text { available. Further research (if } \\
\text { performed) is likely to have an } \\
\text { important impact on our confidence in } \\
\text { the estimate of effect and is likely to } \\
\text { change the estimate. }\end{array}$ \\
\hline $\begin{array}{l}\text { Strong } \\
\text { recommendation } \\
\text { Very low-quality } \\
\text { evidence (very rarely } \\
\text { applicable) }\end{array}$ & $\begin{array}{l}\text { Benefits clearly outweigh } \\
\text { harms and burdens, or } \\
\text { vice versa }\end{array}$ & $\begin{array}{l}\text { Evidence for at least one of } \\
\text { the critical outcomes from } \\
\text { unsystematic clinical } \\
\text { observations or very indirect } \\
\text { evidence }\end{array}$ & $\begin{array}{l}\text { Recommendation may change when } \\
\text { higher quality evidence becomes } \\
\text { available; any estimate of effect, for at } \\
\text { least one critical outcome, is very } \\
\text { uncertain. }\end{array}$ \\
\hline $\begin{array}{l}\text { Weak } \\
\text { recommendation } \\
\text { High-quality evidence }\end{array}$ & $\begin{array}{l}\text { Benefits closely balanced } \\
\text { with harms and burdens }\end{array}$ & $\begin{array}{l}\text { Consistent evidence from } \\
\text { well-performed RCTs or } \\
\text { exceptionally strong evidence } \\
\text { from unbiased observational } \\
\text { studies }\end{array}$ & $\begin{array}{l}\text { The best action may differ depending } \\
\text { on circumstances or patient or } \\
\text { societal values. Further research is } \\
\text { very unlikely to change our } \\
\text { confidence in the estimate of effect. }\end{array}$ \\
\hline
\end{tabular}




\begin{tabular}{|c|c|c|c|}
\hline $\begin{array}{c}\text { Grade of } \\
\text { Recommendation }\end{array}$ & $\begin{array}{l}\text { Clarity of Risk/ } \\
\text { Benefit }\end{array}$ & $\begin{array}{c}\text { Quality of Supporting } \\
\text { Evidence }\end{array}$ & Implications \\
\hline $\begin{array}{l}\text { Weak } \\
\text { recommendation } \\
\text { Moderate-quality } \\
\text { evidence }\end{array}$ & $\begin{array}{l}\text { Benefits closely balanced } \\
\text { with harms and burdens }\end{array}$ & $\begin{array}{l}\text { Evidence from RCTs with } \\
\text { important limitations } \\
\text { (inconsistent results, } \\
\text { methodological flaws, indirect } \\
\text { or imprecise evidence), or } \\
\text { unusually strong evidence } \\
\text { from unbiased observational } \\
\text { studies }\end{array}$ & $\begin{array}{l}\text { Alternative approaches likely to be } \\
\text { better for some patients under some } \\
\text { circumstances. Further research (if } \\
\text { performed) is likely to have an } \\
\text { important impact on our confidence in } \\
\text { the estimate of effect and may change } \\
\text { the estimate. }\end{array}$ \\
\hline $\begin{array}{l}\text { Weak } \\
\text { recommendation } \\
\text { Low-quality evidence }\end{array}$ & $\begin{array}{l}\text { Uncertainty in the } \\
\text { estimates of benefits, } \\
\text { harms, and burdens; } \\
\text { benefits may be closely } \\
\text { balanced with harms and } \\
\text { burdens }\end{array}$ & $\begin{array}{l}\text { Evidence for at least one } \\
\text { critical outcome from } \\
\text { observational studies, from } \\
\text { RCTs with serious flaws, or } \\
\text { indirect evidence }\end{array}$ & $\begin{array}{l}\text { Other alternatives may be equally } \\
\text { reasonable. Further research is very } \\
\text { likely to have an important impact on } \\
\text { our confidence in the estimate of } \\
\text { effect and is likely to change the } \\
\text { estimate. }\end{array}$ \\
\hline $\begin{array}{l}\text { Weak } \\
\text { recommendation } \\
\text { Very low-quality } \\
\text { evidence }\end{array}$ & $\begin{array}{l}\text { Major uncertainty in the } \\
\text { estimates of benefits, } \\
\text { harms, and burdens; } \\
\text { benefits may or may not } \\
\text { be balanced with harms } \\
\text { and burdens }\end{array}$ & $\begin{array}{l}\text { Evidence for at least one } \\
\text { critical outcome from } \\
\text { unsystematic clinical } \\
\text { observations or very indirect } \\
\text { evidence }\end{array}$ & $\begin{array}{l}\text { Other alternatives may be equally } \\
\text { reasonable. Any estimate of effect, for } \\
\text { at least one critical outcome, is very } \\
\text { uncertain. }\end{array}$ \\
\hline
\end{tabular}

Source: Reprinted with permission of the American Thoracic Society. Copyright @ 2012 American Thoracic Society. Schünemann HJ, Jaeschke R, Cook DJ, Bria WF, El-Solh AA, Ernst A, Fahy BF, Gould MK, Horan KL, Krishnan JA, Manthous CA, Maurer JR, McNicholas WT, Oxman AD, Rubenfeld G, Turino GM, Guyatt G; ATS Documents Development and Implementation Committee. An official ATS statement: grading the quality of evidence and strength of recommendations in ATS guidelines and recommendations. Am J Respir Crit Care Med. 2006 Sep 1;174(5):605-14. Official Journal of the American Thoracic Society. ${ }^{29}$

* Exceptionally strong evidence from unbiased observational studies includes: (1) evidence from studies that yield estimates of the treatment effect that are large and consistent; (2) evidence in which all potential biases may be working to underestimate an apparent treatment effect, and therefore, the actual treatment effect is likely to be larger than that suggested by the study data; and (3) evidence in which a dose-response gradient exists

\section{Existing Systematic Reviews and Clinical Practice Guidelines}

The expert panel and methodology team identified existing systematic reviews and clinical practice guidelines that were relevant to the topics of this guideline, even though they were not necessarily specific to people with $\mathrm{SCD}$. If the methodological quality of these resources was found to be appropriate by the methodology team, they were used. Using this external evidence was considered helpful because well-conducted systematic reviews made the process of identifying relevant studies more feasible. In addition, using existing guidelines developed by professional organizations enabled the panel to develop more comprehensive recommendations that addressed specific aspects of care in individuals with SCD. Usually, this external evidence was derived from studies in non-sickle cell patient cohorts because it was felt that they offered more precise and useful inferences than evidence derived from sickle cell patient studies. For example, comparative evidence in the area of pain management in people with SCD was sparse. In this situation, pain management guidelines from individuals with other pain-related conditions proved to be helpful.

The methodology team used the AMSTAR tool to assess the methodological quality of systematic reviews. ${ }^{30}$ Recent well-conducted systematic reviews were identified that addressed hydroxyurea therapy in pediatric and adult patients. ${ }^{6-8}$ The expert panel and methodology team appraised these reviews and conducted additional searches to update the existing systematic review through May 2010 to find evidence for the benefits, harms, 
and barriers of using hydroxyurea. Regarding the management of children with SCD complications, the panel also used recent evidence that had been systematically reviewed. ${ }^{31}$

Existing clinical practice guidelines were considered acceptable if they had prespecified clinical questions, were developed after a comprehensive literature search, had explicit and clear criteria for the inclusion of evidence, and included recommendations that were explicitly linked to the quality of supporting evidence. The expert panel and methodology team used relevant recommendations from the USPSTF, ${ }^{20}$ the Advisory Committee on Immunization Practices (ACIP), ${ }^{22}$ the Centers for Disease Control and Prevention's (CDC) adaptation of the World Health Organization's (WHO's) "Medical Eligibility Criteria for Contraceptive Use,"21 and the American Pain Society's "Guideline for the Management of Acute and Chronic Pain in Sickle-Cell Disease," and "Clinical Guidelines for the Use of Chronic Opioid Therapy in Chronic Noncancer Pain."23,24

\section{Consensus Statements}

The panel believed that, for this guideline document to be most helpful to primary care providers and specialty health care professionals, it needed to be comprehensive. This required that, in areas with minimal existing direct evidence, the panel would provide recommendations based on their and others' expert opinions. Those recommendations are labeled as "consensus." Several different situations, outlined below, led to the use of consensus statements.

\section{Consensus-Panel Expertise}

- Systematic reviews conducted by the methodology team revealed minimal or no supporting evidence (e.g., management of acute hepatic sequestration).

- An adequate systematic review of the literature was not feasible because of anticipated low yield or no yield (e.g., comparative effectiveness of management approaches for individuals with SCD presenting with fever or worsening anemia).

- Recommendations were based on the panel's expert knowledge, practice experience, and ability to extrapolate evidence from non-SCD populations (e.g., management of chronic opioid therapy in chronic SCD pain).

\section{Consensus-Adapted}

- These recommendations were based on the panel's expert knowledge to adapt recommendations derived from existing guidelines and synthesized evidence developed by other professional societies (e.g., management of acute and chronic pain in SCD).

The panel clearly identified these statements as consensus recommendations and acknowledges that these areas represent gaps in the evidence base and areas for future research.

Prior to publication, these guidelines were reviewed by the NHLBI Advisory Council, a separate panel of SCD experts, and the National Blood Disorders Program Coordinating Committee. The guidelines were also posted to the NHLBI Web site for an extensive public review and comment period, which resulted in the submission of more than 1,300 comments from individuals and professional societies. The expert panel and NHLBI staff reviewed each comment or recommendation, many of which resulted in a revision to the guidelines. The guidelines were then reviewed by SCD experts representing three professional societies. 


\section{Clinical Practice Guidelines and the Institute of Medicine}

In April 2011, 12 months after the start of the first of the expert panel report's systematic reviews, the Institute of Medicine (IOM) published "Clinical Practice Guidelines We Can Trust." 32 Although at that point, the panel's processes were already identified and in progress, it was determined that the panel's report was well aligned with the main points that the IOM standards identified as critical to trustworthy guidelines (establishing transparency, managing conflict of interest, guideline development group composition, clinical practice guidelines-systematic review intersection, establishing evidence foundations for and rating strength of recommendations, articulation of recommendations, external review, and updating). Because the panel's work began prior to the release of the IOM standards, it did not include a patient representative, the questions considered were not disseminated for public comment, and at this time, no updates are planned for this guideline document. 


\section{Chapter 2: Health Maintenance for People With Sickle Cell Disease}

\section{Background}

Efforts to coordinate care throughout the lifespan between community settings, primary care practices, specialists' practices, emergency departments, laboratories, and hospitals can significantly improve the health and well-being of individuals with a chronic disease such as SCD. Coordination models such as the medical home can facilitate this coordination. ${ }^{33}$ Individuals with SCD are at high risk for developing multisystem acute and chronic conditions associated with significant morbidity and mortality. Undetected signs and symptoms can begin in early childhood. For example, silent CNS infarcts can present with non-focal signs such as developmental delays or poor or declining school performance in children or changes in social role or work functioning in adults. Throughout their lives, people with SCD should be considered for formal neurocognitive evaluation when assessments reveal any of these concerns. In another example, loss of the kidney's ability to concentrate urine occurs in most individuals with SCD and can result in large urine volumes. In children, this may result in enuresis or bedwetting.

Although treatment of SCD may ameliorate some of these complications, such therapies are often unsuccessful in completely preventing them. Therefore, the next best approach may be screening to identify risk factors and early signs of complications in order to implement measures to reduce morbidity and mortality in individuals with SCD. However, not all screening is useful. The expert panel determined that, in order for evidence that supports screening to be considered high-quality, it needed to meet the following requirements, which were based upon the WHO criteria but modified by the panel: ${ }^{19}$

1. The condition targeted by screening is sufficiently prevalent and clinically significant in persons with SCD.

2. An accurate screening test that identifies the condition is available.

3. There is evidence that early intervention in populations identified by screening is beneficial (e.g., effective therapy exists for preventing or treating a condition).

4. Screening is associated with minimal harm.

5. Screening is cost-effective.

The methodology team conducted systematic reviews of the evidence to synthesize and evaluate relevant research on the utility of commonly used diagnostic tests in individuals with SCD (e.g., electrocardiograms, echocardiograms, pulmonary function tests, kidney function tests, various screening eye exams, brain imaging, and transcranial Doppler (TCD)) and presented the panel with evidence tables which included determinations of the evidence quality.

Sickle cell anemia (SCA) refers to the clinically similar disorders HbSS or HbS $\beta 0$-thalassemia. Sickle cell disease (SCD) refers to all disease genotypes, including SCA and compound heterozygous disorders, such as HbSC, HbSD, and HbS $\beta+$-thalassemia. The carrier state for hemoglobin S (HbAS or sickle cell trait) is not a form of SCD. 
This chapter reviews the available evidence for health maintenance and screening and makes recommendations for children and adults with SCD. In addition to SCD-specific recommendations, this chapter also includes the USPSTF's recommendations on clinical preventive services. ${ }^{20}$ The expert panel also identified recommendations from the Centers for Disease Control and Prevention/World Health Organization (CDC/WHO) report on contraceptive use, which were deemed to be particularly relevant for women with SCD and their partners; these recommendations are included in the latter part of this chapter. ${ }^{21}$ The expert panel reviewed the methods used by the CDC, WHO, and USPSTF, and concluded that the processes used by these organizations were consistent with those used by the panel's methodology team.

\section{Methodology}

Complete information about the methodology for these guidelines can be found in the "Introduction and Methodology" chapter (pages 1-9). The following information, specific to this chapter, supplements the standard methodology that was conducted for all clinical chapters of these guidelines.

A comprehensive study of several databases was conducted, and all human studies in English published from January 1970 to December 2010 that addressed each PICOS question were identified ${ }^{\mathrm{e}}$. A total of 313 studies were included. In the specific instances of antibiotic therapy and blood pressure screening, the review began from database inception through January and July 2011, respectively. In the case of screening, the review went through July 2010. Meta-analysis was only feasible in two areas: (1) efficacy of antibiotic prophylaxis in children and (2) hypertension (HTN) in SCD. The topics of reproductive counseling, contraception, clinical preventive health care services, and immunizations were not searched; recommendations were derived from guidelines published by professional organizations that were based on systematic reviews of broader population groups; these recommendations are labeled "Consensus-Adapted." The key questions for this chapter can be found immediately before the Summary of the Evidence sections for the individual topics.

Detailed information on the evaluated studies as well as the observational and case studies/series referenced can be found in the evidence tables for this chapter (The Use of Prophylactic Antibiotic Therapy in Children With Sickle Cell Disease: A Systematic Review and Meta-Analysis, 2012; Blood Pressure and Sickle Cell Disease: A Systematic Review and Meta-Analysis, 2012; and The Use of Screening Tests in Patients With Sickle Cell Disease: A Systematic Review, 2012) available at http://www.nhlbi.nih.gov/guidelines/scd/index.htm.

\section{Prevention of Invasive Pneumococcal Infection}

\section{Background}

Young children with SCA have a very high risk for septicemia and meningitis in the absence of appropriate prophylaxis. $^{34,35}$ These infections result from defective or absent splenic function that typically has its onset in people with SCA early in the first year of life. Case fatality is high, and the risk is greatest in young people who lack humoral immunity against the specific pneumococcal serotype causing infection. People with HbSC and $\mathrm{HbS} \beta^{+}$-thalassemia have a much lower incidence of life-threatening infection because their spleen function is normal or only minimally impaired during infancy. However, older children and adults with all SCD genotypes are at increased risk for invasive bacterial infection.

\footnotetext{
${ }^{\mathrm{e}}$ An updated search was performed to span the time from June 1, 2010 through July 11, 2014. No additional RCTs were identified that were relevant to this chapter.
} 
Universal newborn screening identifies babies with all forms of SCD, including those with SCA, who are most at risk for invasive pneumococcal infection, and allows for the opportunity to initiate the following three-step prevention strategy: (1) twice-daily prophylactic penicillin beginning in early infancy and continuing through at least age 5; (2) vaccination against pneumococcus and other encapsulated pathogens; and (3) education of those with SCD and their parents and caregivers regarding the need to seek immediate medical attention in the event of fever. ${ }^{34,36}$ Employing such measures has resulted in a greatly reduced incidence of septicemia and meningitis in infants and young children with SCD. ${ }^{15}$

\section{Key Questions}

KQ1. What are the benefits and harms of prophylactic antibiotic use in children with SCD? What are the recommended antibiotic administration regimens and schedules?

\section{Summary of the Evidence}

In addition to the systematic review for these key questions, a meta-analysis was conducted. Three RCTs and one observational study were included. ${ }^{34,36-38}$ The studies enrolled a total of 951 children under the age of 5; of these, 94 percent were HbSS, 5 percent were $\mathrm{HbSC}$, and 1 percent were $\mathrm{HbS}^{0}$-thalassemia. The studies showed that prophylactic antibiotic therapy reduces the risk for pneumococcal infections in children with HbSS disease.

The three RCTs were of moderate methodological quality and compared penicillin to no prophylaxis. The initiation of penicillin prophylaxis was associated with a significant reduction in the risk for developing serious pneumococcal infections (2/105 vs. $13 / 110)$ and a nonsignificant reduction in mortality (0/105 deaths vs. $3 / 110$ deaths; very low-quality evidence due to severe imprecision). A single trial evaluated the consequences of discontinuing penicillin prophylaxis; it suggested that prophylaxis in children who have not had a prior severe pneumococcal infection or a splenectomy may be discontinued at age $5 .{ }^{36}$ Children who continued penicillin had a nonsignificant reduction in systemic pneumococcal infections; there was no effect on mortality. The observational study compared penicillin to spiramycin and demonstrated that penicillin was superior. However, the penicillin group had a higher rate of pneumococcal vaccination, confounding the effect of antibiotics and making strong conclusions difficult. The quality of evidence is very low due to severe imprecision (i.e., small number of events) and methodological limitations. Evidence is lacking in children with genotypes other than $\mathrm{SS}$, even though many clinicians prescribe prophylactic penicillin for them both before and after age 5 . 
1. Administer oral penicillin prophylaxis ( $125 \mathrm{mg}$ for age $<3$ years and $250 \mathrm{mg}$ for age $\geq 3$ years) twice daily until age 5 in all children with HbSS.

(Strong Recommendation, Moderate-Quality Evidence)

2. Discontinue prophylactic penicillin in children with $\mathrm{HbSS}$ at age 5 unless they have had a splenectomy or invasive pneumococcal infection. When discontinuing penicillin prophylaxis at age 5 , it is important to assure that the child has completed the recommended pneumococcal vaccination series, and if not, complete the series immediately.

(Weak Recommendation, Moderate-Quality Evidence)

3. Consider withholding penicillin prophylaxis from children with $\mathrm{HbSC}$ disease and $\mathrm{HbS} \beta^{+}$-thalassemia unless they have had a splenectomy

(Weak Recommendation, Low-Quality Evidence)

4. Assure that people of all ages with SCD have been vaccinated against Streptococcus pneumoniae.* (Strong Recommendation, Moderate-Quality Evidence)

5. Remind people with SCD, their families, and caregivers to seek immediate medical attention whenever fever (temperature greater than $101.3^{\circ} \mathrm{F}$ or $38.5^{\circ} \mathrm{C}$ ) occurs, due to the risk for severe bacterial infections.

(Consensus-Panel Expertise)

* Refer to the "Immunization" section of this chapter for comprehensive information on immunizations.

\section{Screening for Renal Disease}

\section{Background}

Sickle cell nephropathy is a major complication of SCD causing tubular and medullary dysfunction. The most common renal pathologies identified from biopsies are glomerular enlargement, perihilar focal segmental glomerulosclerosis, and global sclerosis. In individuals with SCA, glomerular filtration rate (GFR) and renal plasma flow are increased in childhood, normalize during adolescence, and decline with age. Renal abnormalities can start with defects in urine concentration and acidification ${ }^{39}$ beginning in childhood and progress with age to microalbuminuria, overt proteinuria, glomerulosclerosis, and, in some people, renal failure. Preclinical markers of glomerular damage in other conditions associated with hyperfiltration and hyperperfusion such as diabetes mellitus can be measured as early predictors of progressive renal nephropathy.

Microalbuminuria can be detected long before a positive urine test for proteinuria. Chronic renal failure (CRF) occurs with a variable frequency of 4-20 percent when significant proteinuria or azotemia is present. ${ }^{40}$

\section{Key Question}

KQ2. In asymptomatic individuals with $S C D$, what is the effect of screening for renal disease, by measuring serum creatinine and urine albumin and protein, on mortality and the development of end-stage renal disease (ESRD)?

\section{Summary of the Evidence}

There were no RCTs found that examined the utility of screening for renal disease in individuals with SCD. Fifty-seven observational studies assessed screening with kidney function tests. Nine of these studies were longitudinal and enrolled more than 1,500 subjects but provided no outcomes; the other 48 studies were crosssectional. Potential screening modalities explored in the studies included serum creatinine, creatinine clearance, 
presence of albuminuria, and urine albumin excretion. Overall, the screening studies reported inconsistent results, and the quality of evidence was very low. No data were found on screening intervals.

No consistent differences were found in the presumed "normal" or average creatinine levels between people with and without SCD, and none were found among individuals with different genotypes of SCD. ${ }^{41}$ No studies evaluated the utility of screening or compared the effect of screening versus no screening. In one study of 368 individuals with HbSS, 78 (20.6 percent) had proteinuria, and 17 people (4.6 percent) had renal insufficiency. ${ }^{42}$ Long-term followup revealed that five people (1.9 percent) progressed to ESRD requiring chronic dialysis, and three people ( 0.8 percent) died from complications of renal failure. ${ }^{42}$

The data are limited for early intervention through screening for renal disease in people with SCD. Therefore, the panel chose to consider indirect evidence from non-SCD populations in which pharmacological interventions were beneficial in people with proteinuria. In developing a recommendation for screening for renal disease, the panel placed a low value on the cost and inconvenience of screening (as both are minimal) and a high value on the potential benefits of treating people with signs of early renal impairment.

\section{Recommendations}

1. Screen all individuals with $S C D$, beginning by age 10 , for proteinuria. If the result is negative, repeat screening annually. If the result is positive, perform a first morning void urine albumin-creatinine ratio and if abnormal, consult with or refer to a renal specialist.

(Consensus-Panel Expertise)

\section{Screening for Pulmonary Hypertension}

\section{Background}

Pulmonary hypertension $(\mathrm{PH})$ is defined as an elevation of the resting mean pulmonary arterial pressure ( $\geq 25 \mathrm{mmHg}$ ) as determined by right heart catheterization. ${ }^{43}$ There are several potential etiologies for elevation in mean pulmonary artery pressure in people with SCD. Chronic hemolytic anemias, including SCD, may result in pulmonary vascular changes leading to pulmonary arterial hypertension (PAH), and are placed in Group 1 of the current classification. ${ }^{44}$ This type of pulmonary hypertension may occur in up to 10 percent of those with SCA and accounts for 40-50 percent of all types of PH in SCD. ${ }^{45,46}$ The second most common type of PH in SCD is pulmonary venous hypertension (PVH), which is assigned to Group 2 in the current classification and is associated with an elevated mean pulmonary artery pressure $(\geq 25 \mathrm{mmHg}$ ) but also an elevated pulmonary capillary wedge pressure of $\geq 15 \mathrm{mmHg} .{ }^{43-47}$ This is often associated with left ventricular diastolic dysfunction in SCD. ${ }^{48} \mathrm{PH}$ also occurs in the setting of chronic lung disease, chronic thromboembolic disease, or can be due to unclear or multiple mechanisms (Groups 3, 4, and 5 of the classification, respectively). The main symptoms of PH include shortness of breath during routine activity, such as climbing two flights of stairs; fatigue; lethargy; chest pain; palpitations; syncope; peripheral edema; and decreased appetite. ${ }^{49}$ Distinguishing the etiology of these common and diverse symptoms can be difficult.

Initial assessment for PH has been done with an echocardiography evaluation to estimate pulmonary artery pressure using tricuspid regurgitant jet velocity (TRV), ${ }^{43,45-47}$ but diagnosis requires right heart catheterization and direct measurement of the pulmonary arterial pressure and vaso-reactivity of the vessels. ${ }^{43,45,50}$ Transient elevation in TRV has been observed during acute vaso-occlusive crises in individuals with $\mathrm{SCD},{ }^{51}$ which may not reflect baseline values or represent chronic $\mathrm{PH}$. 
Both elevated TRV and PH are risk factors for premature death in people with SCD. Elevated TRV in adults with SCA is associated with an increased risk for all-cause mortality; however, this is not the case in children with SCA. ${ }^{52-54}$ Observational studies show an increase in hospitalization and mortality in people with all types of SCD who also have PAH documented by right heart catheterization, compared to those who do not (55 percent vs. 21 percent in 10 years). ${ }^{48,52,55,56}$ A commonly associated finding is renal insufficiency. ${ }^{47,57,58}$ The typical age of onset of $\mathrm{PH}$ and prevalence of $\mathrm{PH}$ in people with forms of SCD other than SCA remain unclear. $^{48,52}$

Use of screening (testing asymptomatic individuals) to detect conditions in the presymptomatic stage is generally justified by the ability to impact the course to prevent or reduce morbidity and/or mortality. ${ }^{59}$ Therapies for treating PAH are generally initiated when symptoms are present, with a goal to ameliorate or mitigate these symptoms and improve functional capacity. ${ }^{43,60}$ There are no studies demonstrating a change in mortality or course of $\mathrm{PH}$ when therapies are introduced in the presymptomatic stage. Identification of underlying conditions that may be associated with PH (e.g., scleroderma, HIV) permits treatment of these conditions, but clinical trials demonstrating long-term benefit or reduced mortality are lacking. ${ }^{60}$ Similarly, there are no data yet available to demonstrate that treatment of SCD itself impacts PH or the all-cause mortality associated with an elevated TRV in adults with SCD. Despite this lack of evidence, some groups advocate screening individuals with some diseases, including SCD, when $\mathrm{PH}$ is relatively prevalent. ${ }^{60}$

\section{Key Question}

\section{KQ3. In asymptomatic individuals with SCD, what is the effect of screening for PH on mortality and the development of future cardiac and pulmonary complications?}

\section{Summary of the Evidence}

Eighty-three observational studies are included in the evidence table, and they describe the use of echocardiography as a screening test for PH in people with SCD. Of these studies, 27 were longitudinal, 56 were cross-sectional, and 9 had a comparison group. However, no study evaluated the utility of screening or compared an approach of screening versus no screening on patient outcomes, and there were no data on screening intervals. The overall quality of the data was considered very low.

Bachir and colleagues ${ }^{52}$ showed that rates of presumed PAH based on echocardiography (32-38 percent) were only confirmed in 6 percent of those using right heart catheterization. A similar false-positive rate (68 percent) was noted in a cross-sectional study. ${ }^{60}$ The findings of a study evaluating right heart catheterization-confirmed PAH in persons with SCD showed that 44 percent of the people with PAH died compared to 17 percent of the people without PAH, and the median survival after diagnosis with PAH was 25.6 months (range: $1-46$ ) ${ }^{61}$

Numerous studies showed that the prevalence of elevated TRV in people with SCD ranged from 11 percent to 59 percent. This was much higher than in individuals without SCD and was associated with increased mortality. ${ }^{53,54}$ TRV increased over 2 years of followup, with the greatest increases occurring in the presence of elevated systemic blood pressure. ${ }^{2}$ The echocardiography studies assessing TRV did demonstrate several other abnormalities in individuals with SCD, such as increased left ventricle end-diastolic diameter, increased chamber size of the right ventricle and left atrium, early-diastolic mitral flow velocity and late-diastolic mitral flow velocity, and lowered ejection fraction (EF). ${ }^{63}$ Low EF was also found to be a significant independent risk factor for death. ${ }^{53}$ No studies were found that demonstrated reduction in mortality in SCD using treatments for $\mathrm{PH}$ or to modify the SCD itself. 
Based on the insufficient evidence, the expert panel was unable to make a recommendation for or against screening for PH. However, this does not diminish the importance of evaluating individuals who have symptoms or who have had abnormal echo testing. ${ }^{56}$

\section{Electrocardiogram Screening}

\section{Background}

The electrocardiogram (ECG) may offer diagnostic information to guide clinical decisionmaking. However, there is minimal evidence for the value of obtaining a screening ECG in asymptomatic individuals with or without SCD to detect abnormalities such as prolonged corrected QT interval (QTc), ST-T segment abnormalities, and electrocardiographic cardiac enlargement.

Although observational studies of the prevalence of ECG abnormalities in persons with SCD have been done, they reveal abnormalities that are of unknown clinical significance. Studies found that the prevalence of cardiac enlargement ranged between 22 percent and 76 percent, ${ }^{64,65}$ and ventricular hypertrophy prevalence was found to be between 28 percent and 37 percent, ${ }^{66,67}$ The presence of nonspecific S-T abnormalities was found to be between 18.5 percent and 52 percent. ${ }^{68}$ First-degree atrioventricular block was found in 8 percent of people in one study, ${ }^{67}$ and intraventricular conduction delay was found in 4 percent of people in another study. ${ }^{66}$ Prolonged QTc ranged between 15 percent and 50 percent prevalence. Overall, there was no significant difference in the prevalence of prolonged QTc between people with and without SCD, and the presence of prolonged QTc did not significantly affect mortality. ${ }^{69}$

\section{Key Question}

KQ4. In asymptomatic individuals with SCD, what is the effect of screening with ECG on mortality and the development of future cardiac disease?

\section{Summary of the Evidence}

Fourteen observational studies (4 longitudinal and 10 cross-sectional) described the use of ECG as a screening test in people with SCD; however, all of the studies focused on estimating the prevalence of ECG abnormalities of unknown clinical significance. No study evaluated the utility of ECG screening or compared an approach of ECG screening versus no screening, and no data exist about the effect of obtaining a screening ECG on clinical outcomes in people with SCD. There were no data on screening intervals or diagnostic accuracy of the test, and the overall quality of the evidence supporting screening using ECG was very low.

The USPSTF recommends against routine screening with resting electrocardiography, exercise treadmill test (ETT), or electron-beam computerized tomography (EBCT) scanning for coronary calcium for either the presence of severe coronary artery stenosis (CAS) or the prediction of coronary heart disease (CHD) events in adults at low risk for CHD events (Grade D - moderate to high certainty that the benefits do not outweigh the harms).

\section{Recommendations}

1. Routine ECG screening is not recommended in children and adults with SCD.

(Weak Recommendation, Low-Quality Evidence) 


\section{Screening for Hypertension}

\section{Background}

The "Seventh Report of the Joint National Committee on Prevention, Detection, Evaluation, and Treatment of High Blood Pressure" (JNC 7) ${ }^{70}$ recommends medication for hypertension (HTN), defined as blood pressure (BP) $\geq 140 / 90 \mathrm{mmHg}$; medication for prehypertension (defined as BP 120-139/80-89 mmHg) if accompanied by a comorbidity such as chronic kidney disease or diabetes mellitus; and lifestyle changes for prehypertension not accompanied by a comorbidity. The USPSTF recommends blood pressure screening in all individuals aged 18 or older (Grade A - high certainty that the benefits substantially outweigh the harms). ${ }^{20}$ The "Expert Panel on Integrated Guidelines for Cardiovascular Health and Risk Reduction in Children and Adolescents: Summary Report" ${ }^{71}$ recommends annual blood pressure screening in children aged 3 and older and in younger children with a history of renal, urologic, or cardiac diagnosis or a history of time in the neonatal intensive care unit (ICU). However, the quality and strength of the evidence supporting these recommendations is not provided.

No specific recommendations are made by the USPSTF for individuals with SCD. Individuals with HbSS often have significantly lower diastolic, systolic, and mean BP compared with age/sex-matched healthy controls or individuals with confirmed $\mathrm{HbA} .{ }^{72,73}$ Higher baseline systolic pressure was reported to be a risk factor for silent cerebral infarction in a publication subsequent to the original systematic review. ${ }^{74}$

\section{Key Questions}

KQ5. In people with SCD, what is the effect of screening for HTN on mortality, stroke, and heart disease? What are the acceptable limits for BP parameters above which cardiovascular and cerebrovascular morbidity occur?

\section{Summary of the Evidence}

Thirty-two studies (including 2 RCTs, 14 prospective cohort, 4 retrospective cohort, and 12 cross-sectional studies) involving both adults and children were included and are available in the evidence table. ${ }^{75,76}$ Random effects meta-analysis of these 32 studies was conducted to pool the differences in BP between people with SCD and people without SCD. Individuals with HbSS had significantly lower diastolic, systolic, and mean BP compared with age/sex-matched healthy controls or individuals with confirmed normal hemoglobin. However, no studies were found that prognostically defined "normal" or "elevated" BP for people with SCD at any age. The overall quality of evidence to establish baseline BP in persons with SCD, manage elevated BP, or make prognostic associations was low.

However, in studies involving individuals with SCD both with and without HTN defined according to normal population values, HTN was associated with increased mortality ${ }^{72,77}$ and increased risk for stroke in people with SCA. ${ }^{73,77,78}$ The risk of stroke was also increased for people with SCD even when BP was $\leq 140 / 90 .{ }^{73}$ For people with SCD, HTN (which had varying definitions in the studies) was associated with increased risk for hospitalization $^{78,79}$ and microalbuminuria..$^{80,81}$

There are no published clinical studies in individuals with SCD demonstrating that treatment of blood pressure to specific target values results in improved outcomes. Thus, in developing consensus recommendations for screening for HTN, the panel adapted recommendations from "The Seventh Report of the Joint National Committee on Prevention, Detection, Evaluation, and Treatment of High Blood Pressure" ${ }^{\text {, }}$ (see http:/www.nhlbi.nih.gov/guidelines/hypertension) and the NHLBI report "The Fourth Report on the Diagnosis, 
Evaluation, and Treatment of High Blood Pressure in Children and Adolescents" ${ }^{\nexists 2}$ (see http://www.nhlbi.nih.gov/guidelines/hypertension/hbp ped.htm).

\section{Recommendations}

1. In adults with $S C D$, screen for hypertension and treat to lower systolic blood pressure $\leq 140$ and diastolic blood pressure $\leq 90$ according to "The Seventh Report of the Joint National Committee on Prevention, Detection, Evaluation, and Treatment of High Blood Pressure" (JNC 7).

(Consensus-Adapted)

2. In children with SCD, measure blood pressure, and evaluate and treat hypertension following recommendations from the NHLBl's "Fourth Report on the Diagnosis, Evaluation, and Treatment of High Blood Pressure in Children and Adolescents."

(Consensus-Adapted)

\section{Screening for Retinopathy}

\section{Background}

All individuals with SCD and especially those with $\mathrm{HbSC}$ are at risk for retinal disease due to vaso-occlusion and its resultant ischemia. ${ }^{83,84}$ Proliferative sickle retinopathy (PSR) is of greatest concern because progression is associated with loss of visual acuity. PSR is the development of sea-fan-shaped neovascular fronds in response to local ischemia from peripheral retinal arteriolar occlusion. The fronds can lead to other complications including vitreous hemorrhage and retinal detachment. Prevalence of proliferative retinopathy reported in a contemporary retrospective study of children with SCD was 4.3 percent. ${ }^{85}$ In a study from Jamaica, visual acuity loss attributed to retinopathy was reported in 10 percent of untreated eyes during a 10-year observation period. ${ }^{83}$ Prospective clinical studies have demonstrated the benefit of laser photocoagulation in reducing rates of visual acuity loss and decreasing incidence of vitreous hemorrhage. ${ }^{86-88}$ Surgical intervention may be indicated for certain complications such as vitreous hemorrhage. The onset of sickle retinopathy is in childhood; however, screening requires a dilated eye examination and the ability to do so will vary according to the child's ability to tolerate the exam.

\section{Key Question}

\section{KQ6. In asymptomatic individuals with SCD, are dilated eye examinations useful, and, if so, with what frequency should they be done?}

\section{Summary of the Evidence}

No RCTs of retinal screening in people with SCD were found. Twelve observational studies addressed eye examinations for individuals with SCD, primarily children and adolescents. Of these, five were longitudinal and involved 1,261 individuals, and seven were cross-sectional. Genotypes involved were HbSS, HbSC, and HbS $\beta$-thalassemia. ${ }^{83,84,89-91}$ No studies have been published comparing screening for retinopathy with no screening, nor were data found to evaluate diagnostic accuracy or screening intervals. The overall quality of the screening data were considered low.

In these studies, "eye examinations" varied, and not all included dilation of the pupils. The most comprehensive report describes a 20-year prospective study of an inception cohort of 473 individuals from Jamaica. ${ }^{84}$ Annual eye exams including dilation were performed from age 5, and fluorescein angiography was performed from age 
6 unless patients had an allergy to fluorescein. Fifty-nine of those studied developed proliferative retinopathy. The incidence of retinopathy increased with age, and by the ages of 24 to 26, PSR was present in 43 percent of those with HbSC and 14 percent of people with HbSS. In a retrospective study of 263 children with SCD, including people with $\mathrm{HbSS}$, HBSC, and $\mathrm{HbS} \beta$-thalassemia, the age of onset of retinopathy (proliferative and nonproliferative) was, on average, 12.8 years. ${ }^{89}$

\section{Recommendations}

1. In people with $S C D$, refer to an ophthalmologist for a dilated eye examination to evaluate for retinopathy beginning at age 10.

(Strong Recommendation, Low-Quality Evidence)

2. For people having a normal dilated retinal examination, re-screen at 1-2 year intervals. (Consensus-Panel Expertise)

3. Refer people with suspected retinopathy to a retinal specialist. (Consensus-Panel Expertise)

\section{Screening for Risk of Stroke Using Neuroimaging}

\section{Background}

Stroke is one of the most common and devastating complications of SCD. ${ }^{77,92}$ In the absence of primary stroke prevention, approximately 10 percent of children with SCA will have overt stroke.

This complication presents as sudden onset of weakness, numbness, or other focal neurological signs such as visual disturbances, dysarthria, aphasia, or ataxia. Transient ischemic attacks (TIAs) often precede stroke and may be a harbinger of stroke. ${ }^{77}$ Overt stroke in children is generally secondary to stenosis or occlusion of the internal carotid or middle cerebral artery. Events may be precipitated by acute chest syndrome (ACS), parvovirus infection, or other acute anemic events. ${ }^{77,93}$ Overt stroke recurs in most children with SCA who do not receive chronic transfusions or successful hematopoietic stem cell transplantation. ${ }^{94}$

Transcranial Doppler (TCD) imaging of large intracranial blood vessels to detect increased velocities secondary to stenosis can predict risk of stroke in children with SCA. ${ }^{95}$ Primary stroke prevention using regular blood transfusions in children with such elevated velocities proved successful in the NIH-funded STOP trial. ${ }^{96}$ This approach — which used transfusions for an abnormal TCD velocity $(>200 \mathrm{~cm} / \mathrm{sec})$ - has resulted in a declining incidence of primary overt stroke in children with SCD. ${ }^{97}$ Unfortunately, discontinuation of such transfusions was shown in the STOP-2 trial to result in a high rate of reversion to increased TCD velocities or to overt stroke. $^{98}$ Therefore, such transfusions may be necessary indefinitely.

Adults with SCA also have a high risk of both ischemic and hemorrhagic stroke. ${ }^{99}$ The latter is usually sudden, with severe headache, seizures, and loss of consciousness. The mortality rate is high. Limited data suggest that TCD is not predictive of either ischemic or hemorrhagic stroke in adults. ${ }^{100}$

Performing neuroimaging with MRI often reveals silent cerebral infarcts, atrophy, or other findings in children or adults with SCA who lack signs or symptoms of stroke but who often have a history of transient ischemic episodes and/or cognitive impairment. However, the specific indications for these imaging studies are controversial, and management of abnormal findings is uncertain. 


\section{Key Question}

\section{KQ7. In asymptomatic individuals with SCD, what is the effect of screening with neuroimaging tests (computed tomography (CT) scan, MRI, or TCD) on the risk of stroke?}

\section{Summary of the Evidence}

Fifty observational studies that evaluated screening with CT scan and MRI were identified. These studies examined the prevalence of certain abnormalities such as silent infarcts; however, no studies compared a screening strategy versus no screening, and no study reported a benefit of screening or early detection on important outcomes. Overall, the quality of evidence supporting the use of screening with MRI or CT scan in adults and children was very low.

Two RCTs and 50 observational studies on the use of TCD were included. The two RCTs evaluated the efficacy of early intervention and demonstrated that screening coupled with prophylactic transfusion can markedly reduce the risk of stroke in children with SCA whose cerebral blood flow velocity measurements are considered at high risk. ${ }^{96,101}$ The fifty observational studies enrolled more than 11,000 patients and assessed the use of TCD as a screening test in children with SCD. The quality of evidence supporting screening with TCD was considered moderate to high.

In an observational study of 274 patients, the cumulative incidence of conversion from a normal TCD velocity $(<170 \mathrm{~cm} / \mathrm{sec})$ to a conditional TCD velocity $(170-199 \mathrm{~m} / \mathrm{sec})$ was 18 percent (10-26 percent) within 18 months from the first examination. ${ }^{102}$ Risk of stroke was higher in children with abnormal TCD than in children with normal TCD, conditional TCD, or inadequate TCD examination results. ${ }^{101}$ Children with normal cerebral blood flow had no strokes after 4 years of followup. ${ }^{103}$ No trials were found that addressed the optimal time interval for screening patients with documented normal TCD velocity. Information from a modeling and decision analysis (not a clinical study) suggests that the optimal stroke prevention strategy is annual TCD ultrasonography screening up to age 10, with transfusion for those at high risk until age $18 .{ }^{104}$ No clinical trials have been published evaluating this strategy. Outcome data in the studies that evaluated TCD screening are mainly derived from patients with genotypes $\mathrm{HbSS}$ and $\mathrm{HbS} \beta^{0}$-thalassemia; therefore, it was not possible to infer about the utility of TCD screening in other genotypes.

\section{Recommendations}

1. In children with SCA, screen annually with TCD according to methods employed in the STOP studies, beginning at age 2 and continuing until at least age 16.

(Strong Recommendation, Moderate-Quality Evidence)

2. In children with conditional $(170-199 \mathrm{~cm} / \mathrm{sec})$ or elevated $(>200 \mathrm{~cm} / \mathrm{sec})$ TCD results, refer to a specialist with expertise in chronic transfusion therapy aimed at preventing stroke.

(Strong Recommendation, High-Quality Evidence)

3. In children with genotypes other than SCA (e.g., HbSß$\beta^{+}$-thalassemia or $\mathrm{HbSC}$ ), do not perform screening with $\mathrm{TCD}$. (Strong Recommendation, Low-Quality Evidence)

4. In asymptomatic children with $S C D$, do not perform screening with MRI or CT. (Moderate Recommendation, Low-Quality Evidence)

5. In asymptomatic adults with $S C D$, do not perform screening with neuroimaging (TCD, MRI, or CT). (Moderate Recommendation, Very Low-Quality Evidence) 


\section{Screening for Pulmonary Disease}

\section{Background}

Respiratory conditions are found in children with SCD at a prevalence of 20 percent to 48 percent ${ }^{105-107}$ and are associated with an increased risk of mortality. In a prospective study of 1,963 individuals with SCA followed from birth through adulthood, individuals with SCA and asthma had a more than twofold higher risk of mortality after adjusting for established risk factors. ${ }^{108}$ In assessing asthma characteristics in an observational study of 79 adults with SCD who completed respiratory symptom questionnaires, those who reported recurrent, severe episodes of wheezing $(n=34)$, regardless of asthma, had twice the rates of pain, ACS, decreased lung function, and increased risk of death compared with adults without recurrent, severe wheezing. ${ }^{109}$

Pulmonary function tests (PFTs) provide a method for objectively assessing the function of the respiratory system. Although multiple studies have demonstrated abnormal pulmonary function in children and adults with $\mathrm{SCD}$, little has been reported regarding the meaning of these changes for the functional status or quality of life in people with SCD. No therapies have been suggested to address these changes unless the person is also shown to have another lung disease, such as asthma, chronic obstructive pulmonary disease (COPD), or pulmonary fibrosis. The utility of screening for respiratory disorders in children and adults using PFTs has not been established. A study to characterize the polysomnographic (PSG) findings of children with SCD who displayed behaviors suspicious of sleep disorder $(n=100)$ identified using the Children's Sleep Habit Questionnaire found sleep-disordered breathing (SDB) in 79 percent of the SCD group. Compared to children with obstructive sleep apnea syndrome (OSAS) without medical comorbidities, children with SCD and OSAS experienced nocturnal desaturation with a fourfold increased risk for oxygen desaturation below 85 percent and hypercapnia. ${ }^{110}$ Therefore, routinely taking a thorough respiratory history is valuable to evaluate symptoms that may require further assessment.

\section{Key Question}

\section{KQ8. In asymptomatic individuals with SCD, what is the effect of screening with PFTs on cardiac and pulmonary complications?}

\section{Summary of the Evidence}

Thirty-four studies (11 longitudinal and 23 cross-sectional) described the results of screening using PFTs in people with SCD who had no recognized respiratory symptoms. No study evaluated the utility of screening or compared an approach of screening versus no screening, and there were no data on diagnostic accuracy. Overall, it was unclear whether early intervention was beneficial or whether screening was cost-effective. Screening intervals were not assessed. The supporting quality of evidence was considered low.

The longitudinal and cross-sectional studies enrolled more than 1,500 and 1,700 subjects, respectively. Children with SCA had lower forced expiratory volume at 1 minute $\left(\mathrm{FEV}_{1}\right)$, forced vital capacity (FVC), and forced expiratory flow (FEF) 25-75 and slower lung growth curves $\left(\mathrm{FEV}_{1}\right.$ and $\left.\mathrm{FEV}_{1} / \mathrm{FVC}\right)$ compared to controls. ${ }^{111,112}$ Lung volume, as a percentage of that predicted, was demonstrated to decline with age in children with SCD, similar to the decline noted in children with cystic fibrosis. ${ }^{111,112}$ A cross-sectional study of African American adults with SCA enrolled in the Cooperative Study of Sickle Cell Disease revealed abnormalities in 90 percent of the subjects (279 of 310$)$. The most common abnormality was a restrictive pattern ( 74 percent) with isolated decreased diffusing capacity observed in 13 percent of the patients. ${ }^{113}$ Other studies demonstrated obstructive changes in 15-21 percent of children and adults with SCD, restrictive changes in 22-27 percent of adults with 
$\mathrm{SCD}$, and mixed restrictive/obstructive changes in 6-12 percent of adults with $\mathrm{SCD}{ }^{54,62}$ Compared with controls, people with SCD had lower FVC, $\mathrm{FEV}_{1}$, and peak expiratory flow rate (PEFR). ${ }^{63}$ When corrected for hemoglobin levels, children with SCA compared to controls of similar age had elevated gas transfer per unit lung volume. ${ }^{114}$ People with HbSC also appear to have lung function abnormalities, which are milder than those seen in people with HbSS. ${ }^{115}$ No studies discussed any type of intervention for children or adults with SCD and abnormal lung function.

\section{Recommendations}

1. In children and adults with SCD, assess for signs and symptoms of respiratory problems (such as asthma, COPD, restrictive lung disease, or obstructive sleep apnea) by history and physical examination.

(Consensus-Panel Expertise)

2. In children and adults with SCD found to have signs or symptoms of respiratory problems by history and/or physical examination, further assessment, which includes pulmonary function tests, is recommended to determine the cause and develop a plan to address the problem.

(Consensus-Panel Expertise)

3. Do not screen asymptomatic children and adults with pulmonary function tests.

(Moderate Recommendation, Low-Quality Evidence)

\section{Reproductive Counseling}

\section{Background}

The CDC and its partners released a set of recommendations and goals for preconception health. ${ }^{116}$ They recommend that women and couples think about their goals for having or not having children and how to achieve these goals, known as a "reproductive life plan." These recommendations apply to all women and couples, but, given the increased risk of adverse pregnancy outcomes in $\mathrm{SCD}^{117}$ and the risk of maternal morbidity and mortality, ${ }^{117,118}$ the expert panel determined that several recommendations were particularly relevant for women with SCD and their partners. The "Recommendations" section delineates these.

\section{Heritability In Men and Women With SCD}

People with SCD are at risk for having a child affected with SCD if their partners have SCD, $\beta$-thalassemia trait, or are carriers of other abnormal hemoglobins such as $\mathrm{HbC}$. Women whose partners carry one of these traits can avoid an affected pregnancy by undergoing preimplantation genetic diagnosis (PGD). PGD is testing performed on an embryo during an in-vitro fertilization cycle (see http://www.acog.org/ /media/For\%20Patients/ faq179.pdf?dmc=1\&ts=20130718T1252201251). ${ }^{119}$ Alternatively, after spontaneous conception, prenatal diagnosis of SCD is possible by chorionic villus sampling in the first trimester or by amniocentesis in the second trimester of gestation. ${ }^{115}$

\section{Fetal Anemia Due to Alloimmunization}

Women with SCD are frequently exposed to blood products. The fetuses of women who are alloimmunized are at risk of significant hemolytic anemia or mortality. 


\section{Summary of the Evidence}

\section{Adverse Fetal Outcomes}

Multiple case series and two population studies ${ }^{117,118}$ have documented increased risk of growth restriction, preterm delivery, and stillbirth among women with SCD. Fetal surveillance, which includes growth ultrasounds and antepartum testing (nonstress tests, biophysical profiles, and contraction stress tests), may lead to planned early delivery and can reduce but not eliminate risks.

\section{Risks to the Mother}

Compared to women without SCD, women with SCD are more likely to experience preeclampsia, ${ }^{117,118,120}$ venous thromboembolism, infections, and maternal mortality during pregnancy. ${ }^{118}$ During pregnancy, $40-50$ percent of women with SCD require at least one hospital admission. ${ }^{120-122}$

Although there are no data specifically for women with SCD, the presence of pulmonary hypertension increases the cardiopulmonary demands of gestation. Non-SCD maternal mortality has been reported to be as high as 30-50 percent in women with pulmonary hypertension. ${ }^{123-125}$ Even with current multidisciplinary care, maternal mortality in women with pulmonary hypertension is still reported to be 10 percent. $^{126}$

\section{Recommendations}

Evidence reviews on this topic were not performed by the methodology team. The expert panel based its recommendations on a review of the literature and consensus opinion. ${ }^{116 .}$

\section{Specific Recommendations for Women or Men With SCD}

1. Encourage each woman, man, and couple affected by SCD to have a reproductive life plan. (Consensus-Panel Expertise)

2. As a part of primary care visits, provide risk assessment and educational and health promotion counseling (or refer to individuals with expertise in these disciplines) to all women and men of childbearing age to reduce reproductive risk and improve pregnancy outcomes. Provide contraceptive counseling, if desired, to prevent unintended pregnancy, and if pregnancy is desired, provide preconception counseling.

(Consensus-Panel Expertise)

3. If the partner of a man or woman with SCD has unknown SCD or thalassemia status, refer the partner for hemoglobinopathy screening.

(Consensus-Panel Expertise)

4. After testing, refer couples who are at risk for having a potentially affected fetus and neonate for genetic counseling. (Consensus-Panel Expertise)

\section{Specific Recommendations for Women With SCD}

1. Test women with SCD who have been transfused and are anticipating pregnancy for red cell alloantibodies. (Consensus-Panel Expertise)

2. If a woman has red cell alloantibodies, test her partner for the corresponding red cell antigen(s). (Consensus-Panel Expertise)

3. If the partner tests positive for the corresponding red cell antigen(s), counsel the woman and her partner about the risks of hemolytic disease in the fetus and neonate, how it is monitored, and how it is treated, or refer them to a maternal-fetal specialist who can provide this education.

(Consensus-Panel Expertise 
4. Counsel women with SCD and their partners or refer for counseling about the following:

(Consensus-Panel Expertise)

a. Pregnancy in women with SCD is considered high risk, and there is an increased risk of adverse pregnancy outcomes including fetal (intrauterine) growth restriction, preterm delivery, and stillbirth.

b. Additional fetal surveillance is required during a pregnancy.

c. There are increased risks to a woman's health during pregnancy. These risks include an increased frequency of pain crises and an increased risk of thrombosis, infections, preeclampsia, and death relative to women who do not have SCD.

For women who require chronic opioid therapy during pregnancy, there is an increased risk of neonatal withdrawal in their newborns.

\section{Contraception}

\section{Background}

In women with SCD, regular use of contraception can decrease the health risks associated with an unintended pregnancy. Hormonal contraceptives may also decrease menstrual blood flow, leading to higher hemoglobin levels. Use of progestin-only hormonal contraceptives lowers the risk of thromboembolism compared to use of estrogen-containing contraceptives and has been shown to be safe for women with SCD. ${ }^{127,128}$

Intrauterine devices (IUDs) and intrauterine implants carry modest risks associated with the insertion procedure, while sterilization carries risks associated with the surgical procedure. There is no evidence that IUDs pose an increased risk for women with SCD.

\section{Summary of the Evidence}

Published data about contraception and SCD were reviewed by the WHO prior to their latest publication of "Medical Eligibility Criteria for Contraceptive Use." ${ }^{, 129}$ Eight studies were reviewed. ${ }^{130-137}$ With the exception of one survey, ${ }^{134}$ the studies were small and compared differences in hematologic parameters or numbers of crises in women before and after starting a particular contraceptive, or between women who were or were not using a particular contraceptive. Progestin-only contraceptives were not associated with an increased risk of thrombosis and may have noncontraceptive benefits in terms of fewer crises and improved hematologic parameters. Data were insufficient on combined hormonal contraceptives.

Women with SCD may have additional considerations that need to be taken into account when assessing the safety of contraceptive methods. For example, a history of stroke is a contraindication to combined hormonal contraception, and by age 20, approximately 11 percent of untreated women with SCD have had a clinically apparent stroke; this statistic increases to 24 percent by age $45 .^{77}$

The CDC adapted the WHO's "Medical Eligibility Criteria for Contraceptive Use" for women with SCD, and those criteria are the basis for the panel's recommendations. ${ }^{21}$ 
Evidence reviews on this topic were not performed by the methodology team. Therefore, the expert panel based its recommendations on those developed by the WHO and the CDC.

1. Progestin-only contraceptives (pills, injections, and implants), levonorgestrel IUDs, and barrier methods have no restrictions or concerns for use in women with SCD.

(Consensus-Adapted)

2. If the benefits are considered to outweigh the risks, combined hormonal contraceptives (pills, patches, and rings) may be used in women with SCD.

(Consensus-Adapted)

\section{Clinical Preventive Services}

\section{Background}

People with existing chronic diseases such as SCD may fail to receive some of the recommended clinical preventive services because they and their health care physicians are focused on controlling and preventing problems from SCD and its related complications or other comorbid chronic diseases. Unfortunately, this primary focus on SCD may result in people developing other health problems that could have been prevented or treated at an earlier stage, when complications are less frequent. With this situation in mind, the expert panel has identified important recommendations from the USPSTF that should be followed in the care of newborns, children, adolescents, and adults with SCD.

The USPSTF is an independent panel of non-Federal experts in prevention and evidence-based medicine and is composed of primary care clinicians (such as internists, pediatricians, family physicians, gynecologists/obstetricians, nurses, and health behavior specialists). The USPSTF conducts scientific evidence reviews of a broad range of clinical preventive health care services and develops recommendations for the general population in the United States. These recommendations are published in the form of "Recommendation Statements." The recommendations are aimed at the prevention and early recognition of chronic disease.

We have included only the strong recommendations with high-level evidence from the USPSTF and therefore will not address the strength of recommendation or evidence for each of the recommendations listed in exhibit 5 . (Please note that these include grade A and B recommendations from the USPSTF. For more information, see http://USPreventiveServicesTaskForce.org.) These general clinical preventive services should be provided to the person with SCD within the patient's principal health care site. This could be a primary care provider, a sickle cell specialist, or, in many instances, both working together and communicating with one another.

Recommendations of the USPSTF are updated on an ongoing basis. Health care professionals are encouraged to view the most up-to-date recommendations at any time by visiting either http://USPreventiveServicesTaskForce.org or by utilizing the searchable and downloadable electronic Preventive Services Selector (ePSS) available at http://www.ePSS.ahrq.gov. 


\section{Exhibit 5. Summary of U.S. Preventive Services Task Force's General Recommendations That Are Also Applicable to Persons With Sickle Cell Disease}

\section{Newborns}

\section{The following should be available to all newborns:}

- SCD screening with clinical consideration of confirmatory test within 2 months

- Hypothyroidism screening (primary TSH with T4 backup or primary T4 with TSH backup)

- Hearing loss screening

- Phenylketonuria (PKU) screening

- Prophylactic ocular topical medication for the prevention of gonococcal ophthalmia neonatorum

- Counseling for pregnant women regarding the advantages of breastfeeding (The expert panel notes that current maternal use of hydroxyurea is a contraindication to breastfeeding.)

Source: U.S. Preventive Services Task Force (USPTF). Recommendations [Internet]. Rockville, MD: USPTF; 2010 [updated December 2010; cited 2014 May 30]. Available from: http://www.uspreventiveservicestaskforce.org/recommendations. htm. ${ }^{20}$

\section{Children Aged 3 Months to 12 Years}

All children (aged 3 months to 12 years or as stated) should have:

- Fluoride supplement in those over 6 months of age whose water supply is deficient in fluoride

- Routine iron supplementation for asymptomatic infants aged 6 months to 12 months who are at increased risk for iron deficiency anemia

- Children aged 3 to 5 should receive routine evaluation for amblyopia, strabismus, and defects in visual acuity using visual acuity test, stereoacuity test, cover-uncover test, Hirschberg light reflex test, autorefraction and/or photoscreening.

- Children aged 6 years and older should be screened for obesity. Offer or refer for intensive counseling and behavioral interventions.

- The USPSTF recommends screening for hepatitis $\mathrm{C}$ virus (HCV) infection in persons at high risk for infection.

Source: U.S. Preventive Services Task Force (USPTF). Recommendations [Internet]. Rockville, MD: USPTF; 2010 [updated December 2010; cited 2014 May 30]. Available from: http://www.uspreventiveservicestaskforce.org/recommendations.htm. ${ }^{20}$

\section{Adolescents Aged 12 to 18 Years}

All adolescents (aged 12 to 18 years) should be assessed and offered:

- HIV screening for all sexually active adolescents 15 years of age and older and for younger teens who are at high risk

- Screen for chlamydial infection for all sexually active nonpregnant women aged 24 and younger

- Screen for gonorrhea infection in all sexually active girls at high risk for infection

- The USPSTF recommends screening for hepatitis $\mathrm{C}$ virus (HCV) infection in persons at high risk for infection.

- Offer high intensity behavior counseling to prevent sexually transmitted infections (STIs) for all sexually active adolescents at increased risk for STIs.

- Provide interventions, including education or brief counseling, to prevent initiation of tobacco use in school-aged children and adolescents (Grade B recommendation)

- Depression screening when systems for diagnosis, treatment, and followup are in place

- Counsel children, adolescents, and young adults aged 10 to 24 years who have fair skin about minimizing their exposure to ultraviolet radiation to reduce risk for skin cancer.

- Screen all teens for obesity and refer obese teens for comprehensive, intensive behavioral interventions 


\section{Adults}

\section{Offer all adults:}

- Hepatitis $\mathrm{C}$ virus screening if

- At high risk for infection (e.g., those with multiple transfusions)

- Born between 1945 and 1965 (offer one-time screening)

- Tobacco use screening and counseling (all adults, repeat at each visit for those who are smoking)

- Screening and behavioral counseling interventions to reduce alcohol misuse

- Screen all adults for obesity, and offer or refer patients with a body mass index of $30 \mathrm{~kg} / \mathrm{m}^{2}$ or higher to intensive, multicomponent behavioral interventions.

- Screen for cervical cancer in women ages 21 to 65 years with cytology (Pap smear) every 3 years; an option for women 30 to 65 is a combination of cytology and human papillomavirus (HPV) testing every 5 years

- HIV screening (offer to all and repeatedly offer to high-risk people)

- Hepatitis B screening (for those on transfusion therapy)

- Assess risk for breast cancer and offer to prescribe risk-reducing medications, if appropriate, for women at increased risk

- Breast screening mammography for women aged 50 to 74 years

- Women whose family history is associated with an increased risk for deleterious mutations in BRCA1 or BRCA2 genes should be referred for genetic counseling and, if indicated after counseling, BRCA testing.

- Chlamydial infection screening for all sexually active women $\leq 24$, and for older women at high risk

- Folic acid supplementation should be used whenever considering or at risk of pregnancy to prevent neural tube defects.

- Cardiovascular disease risk screening

- Diabetes screening for people with hypertension

- Lipids: Screen men ages 25 to 35 at high risk and all men $\geq 35$ years. Screen women 20 years or older who are at high risk

- Screen for high blood pressure in adults aged 18 and older (For blood pressure screening recommendations, see page 18)

- Screen adults for colon cancer beginning at age 50 and continuing until age 75

- Depression screening when staff assisted support in place for diagnosis, treatment, and followup

- Osteoporosis screening for women $\geq 65$ years. For women younger than 65 years, screen those whose fracture risk is equivalent or higher to a 65-year-old White woman.

- Sexually transmitted infection counseling for all sexually active adults at high risk

- Gonorrhea screening for sexually active women $<25$ and others at high risk

- One-time ultrasound abdominal aortic aneurysm screening for men who have smoked and are 65 to 75 years old

Source: U.S. Preventive Services Task Force (USPTF). Recommendations [Internet]. Rockville, MD: USPTF; 2010 [updated December 2010; cited 2014 May 30]. Available from: http://www.uspreventiveservicestaskforce.org/recommendations.htm. ${ }^{20}$ 


\section{Pregnant Women}

\section{Offer all pregnant women:}

- Bacteriuria screening (asymptomatic)

- Gonorrhea screening for women $<25$ years old and for older women at high risk

- Hepatitis B screening

- HIV screening

- Syphilis screening

- Chlamydial screening for all pregnant women aged 24 and younger and for older pregnant women who are at increased risk

- Rh compatibility screening

Source: U.S. Preventive Services Task Force (USPTF). Recommendations [Internet]. Rockville, MD: USPTF; 2010 [updated December 2010; cited 2014 May 30]. Available from: http://www.uspreventiveservicestaskforce.org/recommendations. htm. ${ }^{20}$

\section{Immunizations}

\section{Background}

Immunizations are one of the most useful preventive measures available to infants, children, and adults. This benefit should be extended to all individuals regardless of other chronic conditions, unless there is a specific disease-related or personal (e.g., allergy) contraindication. For people with SCD, there are no disease-related contraindications.

\section{Key Question}

\section{KQ9. Which immunizations should be given to people with SCD?}

\section{Summary of the Evidence}

The Advisory Committee on Immunization Practices (ACIP) reviews the evidence for each immunization it recommends. The expert panel determined that the methodology used for those reviews was compatible with its own methodology. Therefore, evidence reviews for this topic were not performed by the methodology team. The expert panel based its recommendations on those made by the ACIP (see exhibit 6). ${ }^{22}$

\section{Recommendations}

Evidence reviews on this topic were not performed by the methodology team. Therefore, the expert panel based its recommendations on those developed by the ACIP (see exhibit 6).

1. All individuals with SCD should receive immunizations according to the ACIP harmonized immunization schedule unless they have a personal contraindication as noted in the ACIP schedule.

(Consensus-Adapted)

2. Because of their increased susceptibility to invasive pneumococcal disease, all infants with SCD should receive the complete series of the 13-valent conjugate pneumococcal vaccine series beginning shortly after birth and the 23-valent pneumococcal polysaccharide vaccine at age 2 years, with a second dose at age 5 years. *

(Consensus-Panel Expertise)

\footnotetext{
* There is strong and clear evidence that pediatric patients should discontinue prophylactic penicillin at age 5 years provided that their immunizations are up to date. However, this would mean they would be at potential risk of infection by invasive pneumococcus if they would have to wait for additional coverage until age 7 . The Expert Panel's experience dictates that giving the second dose at age 5 years ensures adequate coverage.
} 


\section{Exhibit 6. Immunization Recommendations as Adapted from the Advisory Committee on Immunization Practices (ACIP)}

All individuals should be immunized as recommended by the ACIP. The most up-to-date schedule should be followed, as changes can be made up to four times per year. Consult the immunization schedule at:

http://www.cdc.gov/vaccines/schedules. The following immunizations are of special importance or unique to people with SCD as recommended by the ACIP. These recommendations may also change periodically, and the above ACIP recommendations should be consulted for confirmation.

\section{- Pneumococcal (PCV13) vaccine-Children}

- Children aged 6 to 18 years with functional or anatomic asplenia should receive one dose of PCV13.

\section{- Pneumococcal vaccine-naïve Adults}

- $\quad$ Adults aged $\geq 19$ years with functional or anatomic asplenia who have not previously received PCV13 or PPSV23 should receive

- One dose of PCV13 first, followed by a dose of PPSV23 at least 8 weeks later.

- Subsequent doses of PPSV23 should follow current PPSV23 recommendations for adults at high risk.

- A second PPSV23 dose is recommended 5 years after the first PPSV23 dose for persons aged 19-64 years with functional or anatomic asplenia.

- Additionally, those who received PPSV23 before age 65 years for any indication should receive another dose of the vaccine at age 65 years, or later if at least 5 years have elapsed since their previous PPSV23 dose.

- Previous vaccination with PPSV23-Adults

- Adults aged $\geq 19$ years with functional or anatomic asplenia who previously have received $\geq 1$ dose of PPSV23 should

- Be given a PCV13 dose $\geq 1$ year after the last PPSV23 dose was received.

- For those who require additional doses of PPSV23, the first such dose should be given no sooner than 8 weeks after PCV13 and at least 5 years after the most recent dose of PPSV23.

- Hib

- One dose of Hib vaccine for people aged $>5$ years who have SCD if they have not previously received Hib vaccine

- Meningococcal vaccine

- Vaccinate infants at high risk (including those with $S C D$ ) at 2, 4, and 6 months of age, and again at 12 through 15 months with this vaccine, which is generically known as HibMenCY.

- Persons aged 9 months through 55 years at increased risk for meningococcal disease (e.g., adults with anatomic or functional asplenia or persistent complement component deficiencies) should receive MenACWY.

- Children aged 2 months to 6 years should receive an additional dose of MenACWY 3 years after primary immunization; boosters should be repeated every 5 years thereafter.

- Children $\geq 7$ years of age should receive an additional dose of MenACWY 5 years after primary immunization; boosters should be repeated every 5 years thereafter.

Sources: Hib: http://www.mass.gov/eohhs/docs/dph/cdc/immunization/acip-summary-recommended-groups.pdf; ${ }^{138}$ Meningococcal vaccine: http://www.historyofvaccines.org/content/blog/acip-makes-new-tdap-and-meningococcal-vaccinerecommendations; ; ${ }^{139}$ PCV 13 and PPSV 23: Use of 13-valent pneumococcal conjugate vaccine and 23 valent pneumococcal polysaccharide vaccine for adults for immunocompromising conditions: recommendations of the Advisory Committee on Immunization Practices (ACIP). MMWR Morb Mortal Wkly Rep. 2012 Oct 12;61(40):816-9. ${ }^{140}$ 


\section{Chapter 3: Managing Acute Complications of Sickle Cell Disease}

\section{Introduction}

New clinical approaches and treatments ${ }^{34,96,98}$ have increased the survival of people with SCD, but the average lifespan still remains about two to three decades less than for Americans without SCD. ${ }^{17,141}$ The shorter lifespan is due in part to adverse outcomes related to acute SCD complications. The most common complication of SCD is an acute episode of severe pain, hereafter referred to as an acute vaso-occlusive crisis (VOC). A VOC is defined as pain resulting from tissue ischemia caused by vaso-occlusion most commonly in the bone(s) and bone marrow.

In addition to VOCs, other common acute complications of SCD include fever related to infection, acute kidney injury (AKI), hepatobiliary complications, acute anemia, splenic sequestration, acute chest syndrome (ACS), and acute stroke. Individuals with signs or symptoms of these complications require immediate evaluation and treatment to reduce or prevent morbidity and mortality. Priapism and acute ocular conditions such as central retinal artery occlusion (CRAO) also require urgent management to preserve organ function.

This chapter presents recommendations for the evaluation and management of these common acute SCD complications. For each acute complication discussed, information is presented regarding its frequency, common presentation, usual evaluation, and treatment.

\section{Methodology}

Complete information about the methodology for these guidelines can be found in the "Introduction and Methodology" chapter (pages 1-9). The following information, specific to this chapter, supplements the standard methodology that was conducted for all clinical chapters of these guidelines.

A comprehensive study of several databases was conducted, and all human studies in English published from 1970 to July 2010 that addressed each PICOS question were identified. A total of 549 studies of complications were included. ${ }^{f}$ When the literature search found insufficient evidence on a topic (e.g., vaso-occlusive crisis), these topics were supplemented with recommendations derived from other published guidelines by professional organizations, which were based on systematic reviews of broader population groups; these recommendations are labeled "Consensus-Adapted." In the instances of fever, acute anemia, and multisystem organ failure (MSOF), a literature search was not conducted, so the panel relied on their cumulative expertise and knowledge to make recommendations; these recommendations are labeled "Consensus-Panel Expertise." The key questions for this chapter can be found immediately before the Summary of the Evidence sections for the individual topics.

Sickle cell anemia (SCA) refers to the clinically similar disorders HbSS or HbS $\beta 0$-thalassemia. Sickle cell disease (SCD) refers to all disease genotypes, including SCA and compound heterozygous disorders, such as $\mathrm{HbSC}, \mathrm{HbSD}$, and $\mathrm{HbS} \beta+$-thalassemia. The carrier state for hemoglobin S (HbAS or sickle cell trait) is not a form of SCD.

${ }^{\mathrm{f}}$ An updated search was performed to span the time from June 1, 2010 through July 11, 2014. Five additional RCTs were identified, for a total of 549 studies, and a supplemental table reflecting these additions was added to the evidence table document. 
Detailed information on the evaluated studies as well as the observational and case studies/series referenced can be found in the Management of Sickle Cell Disease Complications evidence table available at http:/www.nhlbi.nih.gov/guidelines/scd/index.htm.

\section{Vaso-Occlusive Crisis}

\section{Background}

A VOC is the hallmark acute complication for persons with SCD and manifests as acute severe pain. Although VOCs are typically associated with excruciating pain of sudden onset, some people experience gradual onset of a VOC. Nearly all individuals affected by SCD will experience a VOC during their lifetime. ${ }^{142}$ The first VOC may occur as early as 6 months of age, often presenting as dactylitis, but thereafter VOCs occur with variable frequency. ${ }^{23,143-145}$ VOCs and their accompanying pain most commonly occur in the extremities, chest, and back. When they occur in other sites, they can be confused with, or can be the prodromal stage of, other acute complications (e.g., head (stroke), flank (papillary necrosis), and abdomen (hepatic or splenic sequestration, constipation from opioid toxicity, or another hepatobiliary complication)). The etiology of the pain must be determined in order to rule out potential causes of pain other than an uncomplicated VOC, such as ACS, pneumonia, or other abdominal complications. VOC can still occur in the presence of other complications. There are no tests to rule in or to rule out a VOC; there are only tests that potentially rule out other causes of pain. Persons with the genotypes $\mathrm{HbSS}$ or $\mathrm{HbS} \beta^{0}$-thalassemia are likely to experience more frequent VOCs. Persons with HbAS (commonly referred to as sickle cell trait) do not experience typical VOCs. Individuals with more than three hospitalizations for a VOC in a year are at an increased risk of early death. ${ }^{142,143,146-148}$

Pain management must be guided by patient report of pain severity. No biomarkers or imaging studies can validate pain or assess its severity. The primary management of a VOC is analgesic treatment, typically with opioids. No empirical data exist to indicate that rapid analgesic administration results in better outcomes. However, as patients with VOC present with severe pain and are at risk for other complications, best practice suggests that rapid triage, placement, and administration of analgesics should be encouraged. The Emergency Severity Index (ESI) Version 4 triage system, which is used by more than half of emergency departments in the United States, suggests that persons with SCD be triaged as ESI level 2, a very high priority, and rapid placement be facilitated. ${ }^{149}$

Many specific recommendations for acute VOC management are included in this section that address treatment beyond what is listed in the Key Question (below). The expert panel felt it was important to include current practices that have not yet been validated by evidence, but are currently being used. When made, these recommendations are clearly identified as "Consensus-Panel Expertise." A recommendation is included to guide providers in managing persons who take both long- and short-acting opioids to manage pain at home. There are no empirical data to guide whether or not to continue long-acting opioids when ordering continuous opioids via patient-controlled analgesia (PCA). The decision to continue long-acting oral opioids should be made on an individual basis. In most circumstances, it is advisable to continue oral long-acting opioids including methadone therapy even when ordering continuous opioids via PCA to ensure adequate pain relief while avoiding a break in coverage and preventing withdrawal. Finally, hydration and nonpharmacologic therapy are also very important as is concurrent treatment of itching caused by histamine release. 


\section{Key Question}

KQ10. For adults and children with SCD-related acute pain, what are the most effective acute pain management strategies (including types of analgesics, dose and administration protocols, and other interventions such as inhaled nitrous oxide, oxygen, and transfusion)?

\section{Summary of the Evidence}

Thirty-two RCTs with more than 1,800 people of all ages, 34 observational studies, and 30 case reports were considered eligible. Because many of these studies evaluated pharmacologic agents that did not decrease pain or significantly reduce length of hospital stay (e.g., poloxamer 188, fluosol, vasodilators, methylprednisone, oxygen, urea, and other agents), and which are not approved by the U.S. Food and Drug Administration (e.g., inhaled nitrous oxide, transfusion, etc.), recommendations regarding these agents were not made. One study evaluated the effectiveness of meperidine versus placebo or other opioids and found meperidine more effective than placebo in reducing pain. However, due to the neurotoxicity associated with meperidine, the panel did not make recommendations for its use. Evidence from several RCTs and observational studies ${ }^{76,150-153}$ supports the use of opioid therapy in treating VOCs. Indirect, high-quality evidence from populations without SCD also supports the use of opioids in treating VOCs. A recent report from the American Pain Society (APS) suggests opioids are not effective in treating chronic non-cancer pain. ${ }^{154}$ It is important to understand that an acute VOC is considered acute, not chronic pain, and opioids are indicated and should be used to treat pain. Evidence from RCTs and observational studies supporting the use of nonsteroidal anti-inflammatory drugs (NSAIDs) was conflicting, but overall, the evidence supports their efficacy in reducing pain and decreasing length of hospital stay. Several RCTs and observational studies support the use of around-the-clock dosing of analgesics versus intermittent analgesic administration in treating VOCs. ${ }^{155-161}$

The largest study on this topic, a prospective observational study in Saudi Arabia, included 1,154 people and examined the effect of a pain management protocol. ${ }^{155}$ The study found that around-the-clock analgesic infusions for the first 24 hours after admission were more effective for managing VOCs than "on demand" or patient-requested infusions of analgesics. People treated with around-the-clock analgesics achieved a higher discharge rate within 72 hours of admission (83 percent), compared with people who received intermittent (per patient request) analgesics (71 percent). Other observational studies supported these findings and also suggested a more rapid resolution of VOCs and a strong patient preference for around-the-clock analgesic infusions. The evidence base was insufficient to make specific analgesic dosing recommendations or recommendations for several nonpharmacologic approaches (including oxygen, inhaled nitrous oxide, electrical nerve stimulation, acupuncture, biofeedback, and a day hospital program). In general, the quality of the available evidence was moderate to low.

In addition, the panel and the methodology team appraised the quality of the APS's ${ }^{23}$ guidelines for the management of SCD-related pain and found them to be acceptable. As shown in the "Consensus-Adapted" recommendations below, the panel adapted selected recommendations from the APS guidelines for treatment of SCD pain. Additional recommendations are based upon the experience of the expert panel and are categorized as "Consensus-Panel Expertise." 
The recommendations labeled "consensus" in this section were based on recommendations developed by the APS or on panel expertise. The remaining recommendations are based on the evidence review conducted by the methodology team. These recommendations are intended to be for all settings where patients present with VOC.

1. In adults and children with SCD and pain,

- When indicated, initiate diagnostic evaluation of causes of pain other than a VOC while beginning to treat pain.

(Consensus-Adapted)

2. In adults and children with SCD and a VOC,

- Determine characteristics, associated symptoms, location, and intensity of pain based on patient self-report and observation. If the VOC pain is atypical, investigate other possible etiologies of pain.

(Consensus-Adapted)

Rapidly assess the patient's recent analgesic use (opioid and nonopioid).

(Consensus-Adapted)

Rapidly initiate analgesic therapy within 30 minutes of triage or within 60 minutes of registration.

(Consensus-Panel Expertise)

- Base analgesic selection on pain assessment, associated symptoms, outpatient analgesic use, patient knowledge of effective agents and doses, and past experience with side effects.

(Consensus-Adapted)

3. In adults and children with SCD and a VOC,

- Use an individualized prescribing and monitoring protocol (written by the patient's SCD provider) or an SCD-

specific protocol whenever possible (see exhibit 7 on page 36) to promote rapid, effective, and safe analgesic management and resolution of the VOC.

(Consensus-Panel Expertise)

4. In adults and children with SCD and a VOC associated with mild to moderate pain who report relief with NSAIDS in the absence of contraindications to the use of NSAIDS, continue treatment with NSAIDS.

(Moderate Recommendation, Low-Quality Evidence)

5. In adults and children with SCD and a VOC associated with severe pain, rapidly initiate treatment with parenteral opioids.

(Strong Recommendation, High-Quality Evidence)

6. In adults and children with SCD and a VOC associated with severe pain,

- Calculate the parenteral (IV or subcutaneous) opioid dose based on total daily short-acting opioid dose currently being taken at home to manage the VOC.

(Consensus-Panel Expertise)

- Administer parenteral opioids using the subcutaneous route when intravenous access is difficult.

(Consensus-Panel Expertise)

- Reassess pain and re-administer opioids if necessary for continued severe pain every 15-30 minutes until pain is under control per patient report.

(Consensus-Adapted)

- Maintain or consider escalation of the dose by 25 percent until pain is controlled.

(Consensus-Panel Expertise)

- Reassess after each dose for pain relief and side effects.

(Consensus-Panel Expertise)

Initiate around-the-clock opioid administration by patient-controlled analgesia (PCA) or frequently scheduled doses versus "as requested" (PRN) administration.

(Moderate Recommendation, Low-Quality Evidence) 


\section{Recommendations}

7. If ordering around-the-clock, continuous infusion of opioids via the PCA, carefully consider whether there is a need to withhold long-acting oral opioids to prevent over-sedation.

(Consensus-Panel Expertise)

If demand dosing only is ordered via the PCA, continue use of long-acting oral opioids.

(Consensus-Panel Expertise)

- At discharge, evaluate inpatient analgesic requirements, wean parenteral opioids prior to conversion to oral opioids, and adjust home dose of long- and short-acting opioid prescriptions to prevent opioid withdrawal after discharge.

(Consensus-Panel Expertise)

8. In adults and children with SCD and a VOC, do not use meperidine unless it is the only effective opioid for an individual patient.

(Consensus-Adapted)

9. In adults and children with a VOC, administer oral NSAIDS as an adjuvant analgesic in the absence of contraindications.

(Consensus-Adapted)

10. In adults and children with a VOC who require antihistamines for itching secondary to opioid administration, prescribe agents orally, and do not re-administer with each dose of opioid in the acute VOC management phase. Re-administer every 4 to 6 hours if needed.

(Consensus-Panel Expertise)

11. To reduce the risk of acute chest syndrome in adults and children hospitalized for a VOC,

- Encourage use of incentive spirometry while awake.

(Strong Recommendation, Moderate-Quality Evidence)

- Encourage ambulation and activity as soon as possible.

(Consensus-Panel Expertise)

12. In adults and children with VOC, use adjunctive nonpharmacologic approaches to treat pain such as local heat application and distraction.

(Consensus-Adapted)

13. In euvolemic adults and children with SCD and a VOC who are unable to drink fluids, provide intravenous hydration at no more than maintenance rate to avoid over-hydration.

(Consensus-Adapted)

14. In adults and children with SCD and a VOC being treated with opioids, monitor for excessive sedation by measuring sedation with an objective measurement sedation scale and oxygenation levels.

(Consensus-Panel Expertise)

15. Gradually titrate down parenteral opioids as VOC resolves.

(Consensus-Panel Expertise)

16. In adults and children with SCD and a VOC, do not administer a blood transfusion unless there are other indications for transfusion (see the chapter "Blood Transfusion in the Management of Sickle Cell Disease" in these guidelines).

(Moderate Recommendation, Low-Quality Evidence)

17. In adults and children with SCD and a VOC with an oxygen saturation $<95$ percent on room air, administer oxygen. (Consensus-Panel Expertise) 


\section{Exhibit 7. Acute Pain Algorithm*}

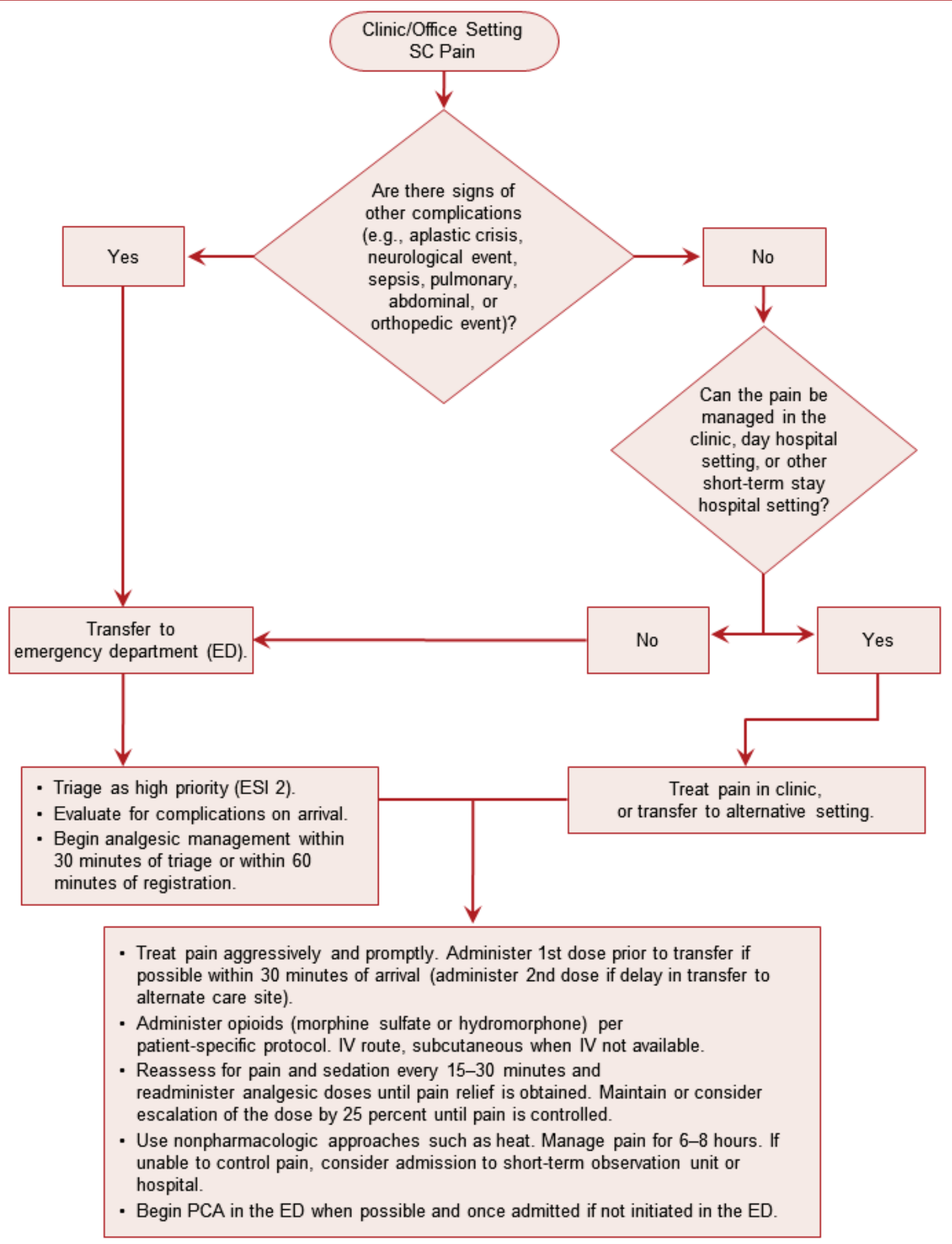

Note: See recommendation 3, page 34 .

* These recommendations are intended to be for all settings where patients present with VOC.

(Consensus-Panel Expertise) 


\section{Fever}

\section{Background}

People with SCA have an increased risk of severe bacterial infection, resulting primarily from reduced or absent splenic function. ${ }^{162}$ By 2 or 3 months of age, as their fetal hemoglobin declines, infants with SCA begin to develop splenic impairment. The result is an extremely high risk of septicemia and meningitis, primarily due to Streptococcus pneumoniae. Although the incidence of invasive pneumococcal infection has declined as a result of prophylactic penicillin and pneumococcal vaccination, febrile illnesses in people with SCD are still considered an emergency due to the possibility of penicillin-resistant organisms and incomplete vaccination status. The risk of such infections continues throughout childhood and to a lesser extent in adults. Serious infections can also affect persons with other forms of SCD (e.g., HbSC and $\mathrm{HbS} \beta^{+}$-thalassemia).

As a presenting symptom, fever heralds many acute and sometimes life-threatening conditions, such as ACS and osteomyelitis. In many cases, the cause of fever is unclear, but because individuals with SCA have a highly increased risk of overwhelming bacterial infection, it is critical that fever alone is taken seriously in these individuals and considered a potential emergency situation. Fever associated with pain should not be considered a VOC until infection is ruled out.

People with SCD who develop fever may have ACS due to diverse organisms (including Mycoplasma) and are also at risk of gram-negative enteric infections involving the urinary tract, hepatobiliary system, or bones. Acute osteomyelitis, another complication associated with fever, may be unifocal or multifocal and may be caused by Staphylococcus aureus, salmonella, or other enteric pathogens. Persons with SCD have normal T cell and B cell function, so the risk of acute infection is generally limited to those micro-organisms mentioned above.

Opportunistic infections are infrequent.

\section{Summary of the Evidence}

An adequate systematic review of the literature with fair sensitivity and specificity for all studies indexed by SCD terms and the symptom of fever was not feasible. A large and nonspecific return of studies with significant heterogeneity, high miss rate, and low-quality evidence (lack of comparative studies) was anticipated. No systematic review was conducted, and the panel used a consensus process to develop a proposed strategy for triaging and promptly managing fever.

Recommendations

1. In people with $S C D$ and a temperature $\geq 101.3^{\circ} \mathrm{F}\left(38.5^{\circ} \mathrm{C}\right)$, immediately evaluate with history and physical examination, complete blood count (CBC) with differential, reticulocyte count, blood culture, and urine culture when urinary tract infection is suspected.

(Consensus-Panel Expertise)

2. In children with $S C D$ and a temperature $\geq 101.3^{\circ} \mathrm{F}\left(38.5^{\circ} \mathrm{C}\right)$, promptly administer ongoing empiric parenteral antibiotics that provide coverage against Streptococcus pneumoniae and gram-negative enteric organisms. Subsequent outpatient management using an oral antibiotic is feasible in people who do not appear ill. (Consensus-Panel Expertise)

3. Hospitalize people with $S C D$ and a temperature $\geq 103.1^{\circ} \mathrm{F}\left(39.5^{\circ} \mathrm{C}\right)$ and who appear ill for close observation and intravenous antibiotic therapy.

(Consensus-Panel Expertise)

4. In people with SCD whose febrile illness is accompanied by shortness of breath, tachypnea, cough, and/or rales, manage according to the preceding recommendations and obtain an immediate chest $\mathrm{x}$ ray to investigate for ACS. (Consensus-Panel Expertise) 
5. In febrile people with SCD who have localized or multifocal bone tenderness, especially when accompanied by erythema and swelling, include bacterial osteomyelitis in the differential diagnosis and manage accordingly.

(Consensus-Panel Expertise)

\section{Acute Renal Failure}

\section{Background}

Acute renal failure (ARF) is defined here as a rapid reduction in renal function manifested by a rise in serum creatinine and reduction in glomerular filtration rate (GFR), with or without a decline in urine output. ARF may be due to pre-renal (e.g., dehydration) or post-renal (e.g., obstruction) insults, or result from intrinsic renal disease (e.g., glomerular injury). ARF may occur during an acute VOC, most often in association with ACS or acute multisystem organ failure (MSOF). ${ }^{163}$

Renal papillary necrosis due to medullary infarction from obstruction of the blood supply in the vasa recta affects up to $15-30$ percent of individuals with SCD. ${ }^{164}$ Signs and symptoms include flank pain and hematuria. When present, fever suggests possible superinfection.

ARF may also occur when individuals with chronic sickle cell nephropathy or other chronic kidney diseases are exposed to nephrotoxic medications (e.g., NSAIDs or intravenous contrast dye) or become dehydrated. People with SCD often display a relative inability to maximally concentrate the urine, resulting in increased vulnerability to pre-renal azotemia.

Due to increased renal tubular secretion of creatinine, serum creatinine values in SCD do not rise until significant renal impairment occurs (GFR of $30 \mathrm{~mL} / \mathrm{min}$ or less). ${ }^{39}$ Since the serum creatinine levels are generally low or low-normal in individuals with SCD, the values in ARF may still be within normal limits even if they have doubled from baseline. It is important to consider non-SCD-related causes of ARF before simply attributing ARF to SCD. ${ }^{165}$

When associated with acute MSOF attributed to diffuse vaso-occlusion, ARF may respond to exchange red blood cell transfusion. ${ }^{163,166}$ However, the benefit of transfusion for other causes of ARF in SCD has not been reported. Acute and chronic renal replacement therapy, including hemodialysis, is well-tolerated by people with SCD and should be used when indicated. ${ }^{163,167}$

\section{Key Question}

\section{KQ11. In people with SCD and ARF, what are the most effective strategies to reduce mortality and the risk of developing end-stage renal disease (ESRD)?}

\section{Summary of the Evidence}

The systematic review did not identify comparative studies to demonstrate the superiority of a particular diagnostic or therapeutic approach to ARF in people with SCD. The literature in this area was mostly descriptive of people who developed renal complications (e.g., hyposthenuria, hematuria, impaired urinary potassium excretion and acidification, tubular and glomerular dysfunction, infection, medullary carcinoma, acute necrosis and renal failure). 
One RCT, six observational studies, and nine case reports addressing both acute and chronic complications were evaluated. There were no RCTs that addressed acute complications and the single RCT addressed chronic complications; acute renal complications were only discussed in five retrospective observational case series. ${ }^{40,168-171}$ No controlled trials or prospective studies addressed the recognition or management of acute renal failure in people with SCD, and few studies addressed evaluation or treatment of renal complications of SCD. The systematic review did not identify any literature to guide diagnostic or management recommendations for renal papillary necrosis. Therefore, management recommendations are based on the application of therapies for ARF from other patient populations to people with SCD as noted in the observational reports.

\section{Recommendations}

1. In the setting of an acute rise in serum creatinine of $\geq 0.3 \mathrm{mg} / \mathrm{dL}$,

- Monitor renal function daily, including serum creatinine and fluid intake/output.

(Consensus-Panel Expertise)

- Avoid potential nephrotoxic drugs and imaging agents.

(Consensus-Panel Expertise)

- Evaluate the patient thoroughly for all potential etiologies in consultation with a nephrologist as needed.

(Consensus-Panel Expertise)

2. Do not give blood transfusions to treat ARF unless there are other indications for transfusion.

(Consensus-Panel Expertise)

3. Use renal replacement therapy (e.g., hemodialysis) when needed for acute renal failure.

(Consensus-Panel Expertise)

\section{Priapism}

\section{Background}

Priapism is a sustained, unwanted painful erection lasting 4 or more hours. Stuttering priapism is the occurrence of multiple self-limited episodes of shorter duration ( $<4$ hours) and can be a harbinger of sustained events. ${ }^{172}$ Priapism is a common complication of SCD, affecting 35 percent of boys and men. ${ }^{173}$ It is usually of the lowflow ischemic type and characterized by pain and a soft glans. Blood aspirated from the corpora cavernosa of the penis is dark, with a low $\mathrm{pO}_{2}, \mathrm{pH}$, and glucose concentration. ${ }^{173}$ Prompt recognition of priapism and initiation of conservative medical management may lead to detumescence and limit the need for more aggressive and invasive intervention. Delayed diagnosis and therapy can result in impotence.

\section{Key Question}

KQ12. In males with SCD presenting with acute priapism, what is the relative efficacy of conservative management, pharmacological management, transfusion, and surgery on the outcomes of detumescence and the incidence of future impotence?

\section{Summary of the Evidence}

Seven observational studies and 39 case reports described priapism in the setting of SCD. Overall, the quality of the evidence in this area was low due to the observational and uncontrolled design of the available studies. 
The observational studies included more than 220 people and studied approaches such as shunts, aspiration, exchange transfusion, hydroxyurea, hormonal therapy (e.g., stilbestrol, finasteride, and leuprolide), bicalutamide, hydralazine, sildenafil, oxygen, and hyperhydration to treat priapism in men and boys with SCD. Results were limited, reporting variable success. ${ }^{174-179}$ Several of the studies highlighted the importance of prompt recognition and initial conservative medical management with analgesics, intravenous fluids, oxygen, and sedation if needed. ${ }^{180-183}$

Red blood cell transfusion therapy was inconsistently associated with improvement in acute priapism. ${ }^{184-193}$ In addition, case reports of acute neurological events following exchange transfusion for priapism further limit enthusiasm for routine adoption of this therapy in the absence of proven benefit. ${ }^{194}$ Both observational studies and case reports found that a variety of subsequent interventions used to treat symptoms that persist after initial conservative medical management appear to result in detumescence and retained potency. These include penile aspiration, ${ }^{195,196}$ corporal irrigation using $\alpha$-adrenergic agents (e.g., pseudoephedrine, epinephrine, etilefrine), ${ }^{197-}$ ${ }^{203}$ and the use of oral agents (e.g., PDE-5 inhibitors, pseudo-ephedrine). ${ }^{204}$ Surgical intervention, including shunting, has been utilized most often after more conservative measures fail, with inconsistent benefit. ${ }^{190,205-209}$

In developing recommendations for the care of males with SCD presenting with acute priapism, the expert panel placed great value on preventing pain and future long-term sequelae.

\section{Recommendations}

1. For an episode of priapism lasting 4 hours or longer, initiate interventions to include

- vigorous oral or intravenous hydration and oral or intravenous analgesia

(Strong Recommendation, Low-Quality Evidence); and

consultation with a urologist who can perform further evaluation and intervention for symptoms which do not remit with initial conservative medical management.

(Consensus-Panel Expertise)

2. Do not use transfusion therapy for immediate treatment of priapism associated with SCD.

(Moderate Recommendation, Low-Quality Evidence)

3. Consult with a hematologist for possible preoperative transfusion if surgical intervention is required.

(Consensus-Panel Expertise)

\section{Hepatobiliary Complications}

\section{Background}

Biliary tract abnormalities are common in people with SCD in general and in those with HbSS in particular. These abnormalities include cholelithiasis, acute cholecystitis, biliary sludge, and acute choledocholithiasis. ${ }^{210,211}$ Hemolysis of any etiology results in increased secreted unconjugated bilirubin that tends to precipitate and leads to gallstones and sludge.

\section{Cholelithiasis and Acute Cholecystitis}

Ultrasound-identified rates of gallstones in people with SCD increase with age from 12 percent in those aged 2 to 4 years to 43 percent by 15 to 18 years of age. ${ }^{212,213}$ In adults with SCD, the prevalence of gallstones can be as high as 70-75 percent. ${ }^{214-217}$ Although gallstones are usually asymptomatic, they can be associated with acute infection and inflammation involving the gallbladder, and they may also lead to obstruction of the cystic or bile ducts and acute pancreatitis. 
Despite the high prevalence of gallstones in people with SCD, acute cholecystitis occurs in less than 10 percent of children and adults with SCD. It can occur with or without the presence of gallstones and can present as severe colicky pain in the right upper quadrant (RUQ) with abdominal tenderness on physical exam. Fever, leukocytosis, nausea, and vomiting are also usually present. Nonvisualization of the gallbladder by 60 minutes after cholescintigraphy is a common radiographic finding.

\section{Choledocholithiasis}

Choledocholithiasis is the presence of gallstones in the common bile duct. Symptoms include dull pain in the RUQ, tender hepatomegaly, and rapidly increasing jaundice. According to a patient survey, choledocholithiasis occurs in less than 5 percent of people with SCD who have asymptomatic gallstones. ${ }^{218,219}$ In symptomatic people, the rate of choledocholithiasis is higher, affecting 20 to 60 percent of people with SCD compared to 15 percent of those without SCD. ${ }^{220,221}$ Endoscopic retrograde cholangiopancreatography (ERCP) and sphincterotomy may be required to remove the offending stones.

\section{Acute Hepatic Sequestration}

Both acute hepatic sequestration (AHS) and acute intrahepatic cholestasis (AIC) (also called sickle cell hepatopathy) are associated with SCD. Each requires consideration in evaluating acute upper abdominal pain in people with SCD.

AHS is marked by hepatic enlargement compared to baseline without other explanation and a $2 \mathrm{~g} / \mathrm{dL}$ or greater decline in hemoglobin concentration. Sequestration of red blood cells often develops over a few hours to a few days, and the resultant stretching of the hepatic capsule is usually painful. AHS appears to be uncommon and may be overlooked unless the size of the liver is closely monitored in cases of acute RUQ pain. About twothirds of people with SCD have mild baseline hepatomegaly, so change in size should be monitored. In AHS, liver function tests are only mildly elevated. Acute hemolysis or other causes of hemoglobin decline should be ruled out. Recurrent episodes may occur. ${ }^{222-225}$

\section{Acute Intrahepatic Cholestasis}

AIC is characterized by the sudden onset of RUQ pain, increasing jaundice, a progressively enlarging and exquisitely tender liver, light-colored stools, and extreme hyperbilirubinemia (both conjugated and unconjugated) usually without urobilinogenuria. Thrombocytopenia and coagulation abnormalities may also be present. The clinical picture suggests cholestatic jaundice or choledocholithiasis but without evidence of common duct obstruction or cholangitis. AIC may prove fatal if not recognized and treated promptly. ${ }^{226-230}$

Diagnostic evaluation may reveal exquisite tenderness in the RUQ with a total serum bilirubin level $>50 \mathrm{mg} / \mathrm{dL}$, hypoalbuminemia, thrombocytopenia, elevated alkaline phosphatase, variable levels of transaminases, coagulopathy with increased prothrombin time (PT), and partial thromboplastin time (PTT) to values more than twice baseline in the absence of accelerated hemolysis or obstruction of the extrahepatic biliary system. ${ }^{226-}$ $229,231,232$

\section{Key Questions}

KQ13. In people with SCD, what is the appropriate management of cholelithiasis and related cholecystitis to resolve symptoms and prevent perioperative complications? What is the most effective treatment strategy for people with SCD presenting with AHS and AIC to reduce mortality and resolve symptoms? 


\section{Summary of the Evidence}

There were no RCTs that evaluated different management strategies for hepatobiliary complications related to SCD. Twenty-five observational studies and 53 case reports were identified and described various hepatobiliary complications associated with SCD. Overall, the quality of the evidence was low due to the observational nature of the studies and the lack of a control or comparison arm in 80 percent of the studies.

The observational studies included more than 900 people and almost uniformly focused on cholelithiasis or acute cholecystitis. One observational study, which followed people with SCD from birth, found that the incidence of cholelithiasis was 30 percent in people with SCA and 11 percent in people with HbSC. ${ }^{233}$ Only 2 percent of the people developed symptoms that required surgical intervention.

In most of the surgical studies, cholecystectomy was shown to be effective and safe in people with SCD and cholelithiasis. When surgically feasible and available, the laparoscopic approach was associated with shorter hospital stay, reduced postoperative pain, and overall lower cost. Other case studies described people with SCD and choledocholithiasis who were treated with both open and endoscopic approaches (i.e., ERCP); however, these data were noncomparative, thus limiting the ability to apply these approaches more generally. ${ }^{234-236}$

The systematic review identified only low-quality literature to guide diagnostic or management approaches for hepatic sequestration or intrahepatic cholestasis. Ahn et al. ${ }^{222}$ described 7 people identified in their institution and 37 people from the literature who had SCD and acute hepatopathy (total serum bilirubin concentration $>13$ $\mathrm{mg} / \mathrm{dL}$ ). Among the 22 severe cases, the mortality rate was 64 percent. Only 2 of 9 people who received exchange transfusion died, whereas 12 of 13 people who did not receive exchange transfusion died. This study likely included people with heterogeneous etiologies of acute liver injury, which limits inference. Other case reports $^{226-229}$ described rare cases of AIC and reported favorable results with using total blood exchange by replacing the removed blood with washed sickle-negative blood and fresh frozen plasma. The quality of the evidence in this area is very low.

\section{Recommendations}

1. Treat acute cholecystitis in children and adults with SCD with antibiotics and surgical consultation. (Consensus-Panel Expertise)

2. Treat asymptomatic gallstones with watchful waiting in children and adults with SCD. In those who develop symptoms specific to gallstones, treat with cholecystectomy. The laparoscopic approach is preferred if surgically feasible and available.

(Strong Recommendation, Moderate-Quality Evidence)

3. Consult with a hematologist or sickle cell expert for possible preoperative transfusion if surgical intervention is required.

(Consensus-Panel Expertise)

4. In children and adults with SCD and signs and symptoms of AHS or AIC, provide hydration, rest, close observation, and consult a sickle cell expert for further management.

(Consensus-Panel Expertise)

5. In children and adults with SCD and signs and symptoms of possible AHS or severe AIC, obtain urgent consultation with a sickle cell disease expert for diagnosis confirmation.

(Consensus-Panel Expertise)

6. In children and adults with SCD with confirmed AHS or severe AIC, perform simple or exchange transfusion. (Consensus-Panel Expertise) 


\section{Acute Anemia}

\section{Background}

Nearly all people with SCD have chronic anemia, but individual baseline hemoglobin values vary widely depending upon hemoglobin genotype ( $\mathrm{HbSS}, \mathrm{HbSC}, \mathrm{HbS} \beta^{+}$-thalassemia, $\mathrm{HbS} \beta^{0}$-thalassemia), current and recent therapies (blood transfusions and hydroxyurea in particular), and other unknown factors. It is important for the patient and his or her primary care provider to know the baseline or "steady state" hemoglobin value to inform ongoing monitoring and management during acute complications. Baseline values are typically 6-8 g/dL for people with SCA, 10-15 g/dL for people with $\mathrm{HbSC}$, and 9-12 g/dL for people with $\mathrm{HbS}^{+}{ }_{-}^{-}$ thalassemia.

Acute anemia, defined as a decline by $2.0 \mathrm{~g} / \mathrm{dL}$ or more in hemoglobin concentration below the patient's baseline value, can have diverse causes. Potential etiologies such as splenic sequestration in a child or an aplastic episode at any age may require urgent evaluation and therapy.

During acute events, the reticulocyte count is an important addition to the CBC to assess whether diminished red blood cell production (low reticulocyte count, as can occur in parvovirus infection resulting in aplastic crisis), accelerated hemolysis, or sequestration in the lungs, spleen, or liver is responsible for the acute anemia.

\section{Aplastic Episode}

An aplastic episode or "crisis" is a common feature of SCD, especially in children with HbSS. ${ }^{237,238}$ The usual clinical picture is gradual onset of fatigue, shortness of breath, and sometimes syncope. Fever is quite common as well. Physical examination may reveal lethargy, rapid heart rate, and occasionally frank heart failure. The hemoglobin value (typically 3-6 g/dL) is usually far below the person's baseline level, and the reticulocyte count is reduced or even zero.

It has been noted that people with SCD rarely have recurrences of aplastic crisis, and several people with SCD in the same household frequently develop aplastic crises simultaneously or sequentially. This pattern suggests an infectious etiology. In the early 1980s, it was shown that parvovirus B19, the cause of fifth disease in young children, is in fact the etiology of these events. ${ }^{238}$ This virus destroys erythroid precursors in the bone marrow, so people with an extremely short red blood cell lifespan such as those with SCA are susceptible to rapid decline in their hemoglobin concentration. Resolution of the aplastic crisis is heralded by marked reticulocytosis and rising hemoglobin concentration, concomitant with the appearance of immunoglobulin $\mathrm{G}(\mathrm{IgG})$ antibodies which neutralize the offending virus. The resulting humoral immunity is lifelong, preventing recurrent events. However, siblings or others with SCD who are exposed to a person with an aplastic crisis in the acute phase are at risk. Aplastic crises are most commonly seen in children with SCA. People with other genotypes, whose hemolysis is less severe, more often have clinically silent events. Occasionally, parvovirus B19 may also be responsible for or contribute to the development of ACS and/or stroke.

\section{Other Causes of Acute Anemia}

Acute splenic sequestration is a major cause of acute anemia, especially in children with SCA. This complication and the recommendations for its management will be described separately (see page 44).

A decline in hemoglobin concentration below the baseline is a common feature of ACS and can be its initial manifestation in a patient experiencing a VOC. Acute anemia may also occur as a result of sequestration of blood in the liver or accelerated hemolysis due to a delayed hemolytic transfusion reaction, septicemia, or another serious infection. Acute blood loss due to papillary necrosis or unrelated to SCD, such as 
gastrointestinal hemorrhage, can also occasionally be responsible for a rapid decline in hemoglobin concentration. Slow but progressive reduction in hemoglobin values should raise concern about renal failure in the older child or adult with SCD.

\section{Summary of the Evidence}

An adequate systematic review of the literature with fair sensitivity and specificity for all studies indexed by SCD terms and the symptom of acute anemia was not feasible. A large and nonspecific return of studies with significant heterogeneity, high miss rate, and low-quality evidence (lack of comparative studies) was anticipated. No systematic evidence review was conducted, and the panel used a consensus process to develop a proposed strategy for triaging and promptly managing acute anemia.

\section{Recommendations}

1. During all acute illnesses in people with $S C D$, obtain a $C B C$ and reticulocyte count, repeat daily in all hospitalized patients, and compare the results with the patient's prior measurements.

(Consensus-Panel Expertise)

2. Assess people with SCD whose hemoglobin concentration is $2 \mathrm{~g} / \mathrm{dL}$ or more below their baseline (or less than $6 \mathrm{~g} / \mathrm{dL}$ when the baseline is unknown) for acute splenic sequestration, an aplastic episode, a delayed hemolytic transfusion reaction, ACS, and infection.

(Consensus-Panel Expertise)

3. Use simple transfusion in people with SCD and acute anemia whose symptoms are due to anemia. (Consensus-Panel Expertise)

4. Perform a $\mathrm{CBC}$ and reticulocyte count promptly and again 7 to 10 days later in siblings and others with SCD who are exposed to a person with an aplastic episode.

(Consensus-Panel Expertise)

5. Manage aplastic events with immediate red blood cell transfusion aimed at restoring the hemoglobin to a safe (not necessarily baseline) value. Isolation of hospitalized patients (droplet precautions) is required to prevent spread of the parvovirus B19 to pregnant women and others with SCD or compromised immunity.

(Consensus-Panel Expertise)

\section{Splenic Sequestration}

\section{Background}

Splenic sequestration is defined as sudden enlargement of the spleen and reduction in hemoglobin concentration by at least $2 \mathrm{~g} / \mathrm{dL}$ below the baseline value. It is a major cause of acute anemia. During splenic sequestration, the reticulocyte count and circulating nucleated red blood cells are usually elevated, and the platelet count is generally decreased because both red cells and platelets are trapped in the spleen. Sequestration usually develops without warning or known cause. It may occur as early as several months of age, ${ }^{239}$ although it is more typical in children between the ages of 1 and 4 years old. Parents may note an enlarging mass in the left upper quadrant. Involution and autoinfarction of the spleen usually occurs by age 5, so sequestration events are less common in older children and adults with $\mathrm{HbSS}$. In people with $\mathrm{HbSS}$, the lifetime prevalence of acute splenic sequestration has been reported to be between 7 percent and 30 percent. In people with $\mathrm{HbSC}$ and $\mathrm{HbS} \beta^{+}$thalassemia, splenic sequestration often occurs later in childhood or even during the adult years. Splenic sequestration in older patients is often accompanied by severe pain from splenic infarction, which can be documented by imaging studies. ${ }^{240}$ 
Some people with SCD have a chronically enlarged spleen and may develop hypersplenism. This presents as a reduction in the white blood cell and platelet counts in addition to acute anemia. Such people are particularly prone to develop acute sequestration events. ${ }^{239}$

In infants with HbSS, splenic sequestration may present acutely with severe anemia and hypovolemic shock. In older people, it may occur more insidiously. Although usual care for splenic sequestration consists of blood transfusion aimed at partial correction of the anemia, excessive transfusion (to hemoglobin values over $8 \mathrm{~g} / \mathrm{dL}$ ) should be avoided, as the sequestered erythrocytes in the enlarged spleen typically reenter the circulation several days later. The result could be hyperviscosity due to an excessively high hemoglobin concentration.

People with splenic sequestration must be monitored for recurrences. Thus, parents and patients are instructed to monitor splenic size and immediately report any marked increase above baseline. People with recurrent sequestration or a single life-threatening acute sequestration event most commonly have a splenectomy. Most people with chronic splenic sequestration accompanied by local pain and hypersplenism are also managed with splenectomy. Splenectomy for splenic sequestration does not further increase the risk of death or bacteremia ${ }^{241}$ since most patients are already functionally asplenic. Regularly scheduled transfusions aimed at avoiding the need for subsequent splenectomy have not been proven to be beneficial. ${ }^{242}$

\section{Key Question}

\section{KQ14. In people with SCD with acute anemia and splenic sequestration or hypersplenism, what are the most} effective strategies to reduce mortality, correct anemia, and prevent recurrence?

\section{Summary of the Evidence}

No RCTs were found that evaluated the treatment of splenic complications in SCD. Twenty observational studies (involving more than 950 people) and 39 case reports described various splenic complications in SCD. Reported complications in these observational studies included: splenic sequestration $(n=16)$, hypersplenism $(n=3)$, splenic abscess $(n=2)$, and functional asplenia/splenic auto infarction $(n=2)$. Overall benefits were reported for transfusion and splenectomy; however, since 75 percent of the studies had no comparative arm, the general quality of the evidence was considered low.

Only four studies, all involving children, had a comparative design. ${ }^{242-245}$ The first compared an intensive transfusion program (to achieve an $\mathrm{HbS}$ concentration $<20$ percent) to a conventional transfusion program in children with prior stroke. ${ }^{243}$ It reported the finding of normal or increased splenic size and improved function in the population receiving intensive transfusion, while all people receiving fewer transfusions had decreased splenic function (functional asplenia). A second study assessed three options for treating splenic sequestration: prompt splenectomy, a short-term transfusion program, or observation. Short-term transfusion was equivalent to observation and therefore of limited benefit in preventing recurrent splenic sequestration. ${ }^{242}$ The third comparative study did not report group-specific outcomes but rather overall mortality rates. ${ }^{244}$ The final comparative study included people with SCD with various splenic complications (splenic sequestration, hypersplenism) and compared outcomes in people who received splenectomy and those who did not. ${ }^{245}$ The remaining studies described splenectomy $(n=13)$, transfusion $(n=3)$, an age-dependent approach $(n=1),{ }^{246}$ and hydroxyurea $(n=1){ }^{247}$ The splenectomy studies reported favorable outcomes following the surgery. Infection rates after splenectomy did not increase. Transfusion was reported to be effective in treating acute splenic sequestration. ${ }^{248,249}$ 
1. In people with hypovolemia due to severe acute splenic sequestration, immediately provide IV fluid resuscitation. (Strong Recommendation, Low-Quality Evidence)

2. In consultation with a sickle cell expert, transfuse people who have acute splenic sequestration and severe anemia to raise the hemoglobin to a stable level, while avoiding over-transfusion.

(Strong Recommendation, Low Quality Evidence)

3. In consultation with a sickle cell expert, address the performance and timing of splenectomy in people with recurrent acute splenic sequestration or symptomatic hypersplenism.

(Moderate Recommendation, Low-Quality Evidence)

\section{Acute Chest Syndrome}

\section{Background}

ACS is one of the most common and serious acute complications of SCD. ${ }^{250-252}$ It is the second most frequent reason for hospitalization in children and adults with SCD and the most common cause of death. Clinically, ACS resembles pneumonia and can develop suddenly or insidiously, during hospitalization for a VOC, or after a surgical procedure, especially one involving the abdomen. ACS occurs with increased frequency in people with asthma or prior ACS events. The clinical, laboratory, and radiographic features of ACS - as well as its management and outcome-were comprehensively assessed in a landmark study performed by the National Acute Chest Syndrome Study Group. ${ }^{251}$

A person with ACS typically has sudden onset of signs and symptoms of lower respiratory tract disease (e.g., some combination of cough, shortness of breath, retractions, rales, etc.) and a new pulmonary infiltrate on chest radiograph. In the early stages of ACS, the clinical manifestations can be subtle. Children usually have fever and upper or middle lobe involvement, whereas adults are often afebrile and present with multilobe disease. The most common well-defined etiology is infection (e.g., viral, bacterial, chlamydia, or Mycoplasma), but the complication may also result from bone marrow fat embolism, intrapulmonary aggregates of sickled cells, atelectasis, or pulmonary edema. In many cases, the specific cause or inciting factor is not apparent. There are no distinctive laboratory features of ACS, although the hemoglobin concentration often declines sharply below the patient's baseline value. In brief, what would be considered pneumonia in a person without SCD usually fulfills the criteria for ACS.

People with ACS generally improve within several days but some develop rapid respiratory failure and/or involvement of other organs such as the brain, kidneys, and liver. This latter complication is known as "multisystem organ failure (MSOF)" (see page 50). Treatment of ACS may include broad spectrum antibiotics, supplemental oxygen, bronchodilators, and blood transfusions. Markers of an impending severe course of ACS are multilobe disease, increased work of breathing, inability to maintain oxygen saturation above 95 percent even with supplemental oxygen, and pleural effusions. Exchange transfusion is often necessary in such circumstances. The therapeutic role of corticosteroids and other anti-inflammatory agents is uncertain and requires further study. ${ }^{253}$ Repeated episodes of ACS occur in many patients and can contribute to development of chronic lung disease.

ACS during a hospital admission for an acute VOC may be prevented by incentive spirometry every 2-4 hours while awake. 


\section{Key Question}

KQ15. In people with SCD and ACS, what is the most effective treatment (among transfusion, exchange transfusion, supportive therapy, steroids, and/or antibiotics) to reduce mortality, resolve pain, and prevent clinical deterioration?

\section{Summary of the Evidence}

One RCT, 27 observational studies, and 45 case reports described sickle cell-related ACS. The overall quality of evidence was very low for all interventions except the use of opioids.

The single RCT enrolled 38 children and found that dexamethasone compared to placebo decreased the mean hospital stay (from 80 to 47 hours), the need for transfusions (from 47 percent to 9 percent), the number of administered opioid doses (from a mean of 20 to a mean of 2.5), and clinical deterioration (defined as an increase in oxygen requirements and respiratory rate). ${ }^{254}$ Participants and investigators were blinded, allocation was concealed, and the study did not report any baseline imbalances. This short-term benefit, however, was not demonstrated to persist when examined by larger observational studies with longer followup. The largest of these studies was done in 2009 and retrospectively evaluated more than 3,000 people (more than 5,000 admissions). ${ }^{255}$ After adjustment for propensity scores and hospital case mix, the study demonstrated a significant increase in the length of hospitalization in people who received corticosteroids as part of their ACS management. Other studies showed increased adverse effects related to steroids.

The remaining observational studies described benefits of other therapies for ACS (e.g., supportive treatment including oxygen supplementation, mechanical ventilation, pain management, hydration, antibiotics, and simple or exchange transfusion). The quality of these studies was low due to the noncomparative nature of their design. Studies that evaluated antibiotics did not demonstrate a significant effect on patient-important outcomes. Multiple observational studies evaluated opiates in ACS. In one, nalbuphine hydrochloride reduced the incidence of ACS compared to morphine (12 percent vs. 29 percent) and also reduced hospital stay. ${ }^{256}$ In the remaining studies, opiates clearly reduced pain but without other effects on the clinical course of ACS. Transfusion studies in ACS showed conflicting results. In one study, length of hospital stay was similar between simple transfusion and exchange transfusion, and ICU stay was longer in the exchange group (5.6 days vs. 2.6 days). ${ }^{257}$ Another study found significant correlation between exchange transfusion and fewer days of hospitalization and oxygen requirement. ${ }^{258}$ In these and other transfusion studies, sicker patients were more likely to receive exchange transfusion, which indicates a clear selection bias. 
1. Evaluate people with SCD who develop acute onset of lower respiratory tract disease signs and/or symptoms (cough, shortness of breath, tachypnea, retractions, or wheezing) with or without fever for ACS. This should include a chest $x$ ray and measurement of oxygen saturation by pulse oximetry.

(Consensus-Panel Expertise)

2. Hospitalize people with ACS. (Consensus-Panel Expertise)

3. Treat people with SCD who have ACS with an intravenous cephalosporin, an oral macrolide antibiotic, supplemental oxygen (to maintain oxygen saturation of greater than 95 percent), and close monitoring for bronchospasm, acute anemia, and hypoxemia.

(Strong Recommendation, Low-Quality Evidence)

4. In people with SCA, give simple blood transfusion ( $10 \mathrm{~mL} / \mathrm{kg}$ red blood cells) to improve oxygen carrying capacity to people with symptomatic ACS whose hemoglobin concentration is $>1.0 \mathrm{~g} / \mathrm{dL}$ below baseline. If baseline hemoglobin is $9 \mathrm{~g} / \mathrm{dL}$ or higher, simple blood transfusion may not be required.

(Weak Recommendation, Low-Quality Evidence)

5. In people with $\mathrm{HbSC}$ disease or $\mathrm{HbS}^{+}$-thalassemia with $\mathrm{ACS}$, decisions about transfusion should be made in consultation with an SCD expert.

(Strong Recommendation, Low-Quality Evidence)

6. In all persons with $S C D$, perform urgent exchange transfusion-with consultation from hematology, critical care, and/or apheresis specialists-when there is rapid progression of ACS as manifested by oxygen saturation below 90 percent despite supplemental oxygen, increasing respiratory distress, progressive pulmonary infiltrates, and/or decline in hemoglobin concentration despite simple transfusion.

(Strong Recommendation, Low-Quality Evidence)

7. Encourage use of incentive spirometry while awake. (Strong Recommendation, Moderate-Quality Evidence)

\section{Acute Stroke}

\section{Background}

Stroke is one of the most common and devastating complications of SCD. ${ }^{77}$ In the absence of primary stroke prevention, approximately 10 percent of children with HbSS will have overt strokes. This complication presents as sudden onset of weakness, aphasia, and sometimes seizures or coma and results in adverse motor and cognitive sequelae. Transient ischemic attack often precedes stroke, even in children, but neuroimaging is negative and not predictive of stroke. In the absence of primary stroke prevention, an additional 20 to 35 percent of children with HbSS have silent cerebral infarcts, which can cause cognitive decline and predispose them to additional silent infarcts and to overt strokes. ${ }^{259}$

Overt stroke is generally secondary to stenosis or occlusion of the internal carotid or middle cerebral artery, but events may be precipitated by ACS, parvovirus infection, or other acute anemic events. ${ }^{77,93}$ In the absence of secondary prevention measures such as a chronic transfusion program or hematopoietic stem cell transplantation, recurrence rates have been shown to range between 46 and 90 percent in children with SCD. ${ }^{94}$ People of all ages with $\mathrm{HbSC}$ and $\mathrm{HbS} \beta^{+}$-thalassemia infrequently have overt CNS events. ${ }^{77}$

Primary stroke prevention using regular blood transfusions in children shown to be at high risk of stroke by TCD screening has led to declines in the incidence of stroke in children with SCD. ${ }^{97}$ Although high-quality 
studies have been done on primary stroke prevention in children, few studies have examined secondary stroke prevention.

Adults with HbSS also have a high risk of both ischemic and hemorrhagic stroke. The latter is usually sudden and is accompanied by severe headache and loss of consciousness. The mortality rate is high. Limited data suggest that TCD is not predictive of stroke risk in adults. This section of the guidelines addresses the management of acute stroke and the prevention of stroke recurrence (i.e., secondary prevention).

\section{Key Question}

KQ16. In people with SCD presenting with acute stroke, what is the most effective treatment strategy (transfusion, thrombolytics, hydroxyurea, or other therapies) to reduce mortality, preserve neurological function, and reduce recurrence rates?

\section{Summary of the Evidence}

The systematic review of the literature did not identify comparative studies that evaluated different management strategies to reduce mortality or improve neurologic outcomes of acute stroke in people with SCD. Therefore, the panel based their initial management recommendations on the principles of stroke management in patients without SCD and on their clinical expertise and provided consensus statements.

The systematic review identified seven observational studies ${ }^{260-266}$ that reported primarily on the effect of transfusion on preventing recurrent stroke (secondary stroke prevention). Two studies ${ }^{262,263}$ reported on the outcomes of stopping chronic transfusion therapy in children who have had prior stroke. There were a total of 20 patients in these studies, and 12 had recurrent central nervous system (CNS) events after discontinuing transfusions. Hulbert et al. ${ }^{266}$ conducted a small retrospective study in 52 children presenting within 24 hours of stroke onset and demonstrated that recurrent stroke occurred in 57 percent ( 8 of 14) of patients treated with simple transfusion, compared with 21 percent ( 8 of 38) of those treated with exchange transfusion. The study by Russell et al. ${ }^{261}$ included 35 children with SCD. Without transfusion, arterial changes documented by arteriography progressed in all four patients who had disease of multiple arteries. After transfusion, vessel changes stabilized. Two of the observational studies reported on long-term outcomes of chronic transfusion. One study followed 60 subjects for a median duration of 36 months, and recurrent strokes were documented in 8 subjects. ${ }^{265}$ The other study ${ }^{264}$ followed 111 patients and found 1.9 events per 100 patient-years, despite longterm transfusions, thus concluding that the risk of recurrent stroke is decreased but not eliminated by regular blood transfusion therapy. The final study ${ }^{260}$ looked at changing the pretransfusion goal of maintaining an $\mathrm{HbS}$ of $<30$ percent to a goal of 50 percent. The median duration of followup was 84 months, and none of the 15 patients studied had a recurrent cerebral infarction during 1,023 patient-months in which the target pretransfusion $\mathrm{HbS}$ was 50 percent. These preliminary single-institution findings were then tested in the prospective Stroke With Transfusions Changing to Hydroxyurea (SWiTCH) multicenter phase 3 clinical trial. Children with previous stroke and iron overload were randomized to receive either continued transfusions with iron chelation (standard arm) or hydroxyurea with phlebotomy (alternative arm). The SWiTCH trial had a noninferiority design, ${ }^{\mathrm{g}}$ with a composite primary end point consisting of recurrent stroke and liver iron concentration. ${ }^{267}$ At interim data analysis, there were seven (7/67) strokes on the alternative arm and none $(0 / 66)$ on the standard arm; this was still within the noninferiority stroke margin, but equivalent liver iron

${ }^{\mathrm{g}}$ A noninferiority trial is a classification of RCT. This type of trial aims to determine whether a new treatment is no less effective than a reference treatment using statistical significance. 
content between treatment arms, indicating futility for the composite study end point. Accordingly, the study was closed, and the authors concluded that transfusions and chelation remain a better way to manage children with SCA, stroke, and iron overload. ${ }^{268}$

In addition to the use of transfusion for secondary stroke prevention, the systematic review identified three small observational studies that evaluated the role of hydroxyurea. ${ }^{94,269,270}$ The studies enrolled a total of 56 children with a history of stroke who were treated with hydroxyurea. The largest of these studies ${ }^{270}$ included 35 children with prior stroke who were discontinued from chronic transfusion therapy. Children were followed on average 42 months with an average hydroxyurea dose of $26.7 \mathrm{mg} / \mathrm{kg} / \mathrm{d}$. The stroke recurrence rate for the whole cohort was 5.7 events/100 patient-years, but for children who overlapped transfusion therapy with hydroxyurea treatment, the event rate was 3.6/100 patient-years. The two smaller studies ${ }^{94,269}$ showed similar results that were consistent with reduction of stroke recurrence associated with using hydroxyurea. The quality of this evidence was low due to imprecision (small sample size) and the uncontrolled nature of the studies.

\section{Recommendations}

1. In people with SCD who present with severe headache, altered level of consciousness, seizures, speech problems, and/or paralysis, evaluate for acute stroke by seeking neurologic consultation and performing an urgent head computerized tomography (CT) scan followed by magnetic resonance imaging (MRI) and magnetic resonance angiography (MRA) if available.

(Consensus-Panel Expertise)

2. In consultation with a sickle cell expert, perform exchange transfusion in people with SCD who develop acute stroke confirmed by neuroimaging.

(Consensus-Panel Expertise)

3. Initiate prompt evaluation, including neurologic consultation and neuroimaging studies, in people with SCD who have mild, subtle, or recent history of signs or symptoms consistent with transient ischemic attack.

(Consensus-Panel Expertise)

4. In children and adults who have had a stroke, initiate a program of monthly simple or exchange transfusions. (Moderate Strength, Low-Quality Evidence)

5. In children and adults who have had a stroke, if it is not possible to implement a transfusion program, initiate hydroxyurea therapy.

(Moderate Strength, Low-Quality Evidence)

\section{Multisystem Organ Failure}

\section{Background}

Multisystem organ failure (MSOF) is a severe and life-threatening complication usually associated with a VOC and characterized by failure of the lungs, liver, and/or kidneys. ${ }^{163}$ MSOF may occur after several days of hospitalization and treatment for a severe VOC, often when pain is beginning to improve. In most cases, patients do not have a history of chronic organ failure. Deterioration is rapid and unexpected. It is usually associated with fever, a rapid decline in hemoglobin concentration and platelet count, and nonfocal encephalopathy. Acute respiratory failure is usually associated with development of ACS. Hepatic failure is associated with marked elevations in total and direct bilirubin, liver enzymes, and blood coagulation screening tests. Acute renal failure is associated with a rapid elevation of serum creatinine, with or without the presence of oliguria and hyperkalemia. Rapid diagnosis and treatment of MSOF is necessary to prevent death. 


\section{Summary of the Evidence}

An adequate systematic review of the literature with fair sensitivity and specificity for all studies indexed by SCD terms and "multisystem organ failure" was not feasible. No systematic review was conducted, and the panel used a consensus process to develop a proposed strategy for triaging and promptly managing MSOF.

\section{Recommendations}

1. In people with SCD who exhibit severe deterioration during a VOC, immediately evaluate for potential MSOF. (Consensus-Panel Expertise)

2. In people with SCD and respiratory failure, support respiratory status with supplemental oxygenation and mechanical ventilation when needed.

(Consensus-Panel Expertise)

3. Use renal replacement therapy (e.g., hemodialysis) when needed for acute renal failure.

(Consensus-Panel Expertise)

4. In people with SCD and MSOF, immediately initiate either simple or exchange transfusion in consultation with a sickle cell expert or hematologist.

(Consensus-Panel Expertise)

\section{Acute Ocular Conditions}

\section{Background}

In persons with SCD, acute ocular complications may occur secondary to trauma, infection, vaso-occlusive episodes leading to occlusion of the eye vasculature, or progression of proliferative sickle retinopathy (PSR). All may have devastating consequences including permanent loss of vision. Hyphema, central retinal artery occlusion (CRAO), orbital and periorbital infections, orbital infarction, and orbital compression syndrome (OCS) all require urgent or emergent assessment and therapy. Although late-stage changes associated with PSR such as nonclearing vitreous hemorrhage and retinal detachment may present with acute visual symptoms, these complications are more fully discussed in the "Managing Chronic Complications of Sickle Cell Disease" chapter of these guidelines.

Hyphema - the presence of blood in the ocular anterior chamber - is often due to blunt injury trauma and typically presents with hemorrhage covering the lower part of the iris and visual abnormalities such as floaters and flashers, light sensitivity, and blurry vision. In persons with SCD, and even in healthy individuals with sickle cell trait, hyphema is especially dangerous due to the hypoxic and acidotic nature of the anterior chamber, which promotes sickling of red blood cells in the aqueous humor. This in turn prevents outflow of sickled erythrocytes and aqueous humor through the trabecular meshwork of the eye and increases pressure in the entire eye. Blood flow in the central retinal artery in the presence of high intraocular pressure (IOP) may result in CRAO and infarction of the optic nerve. Elevated IOP $^{271,272}$ is poorly tolerated in people with SCD. The size of the hyphema is poorly correlated with the risk of visual loss. ${ }^{271-273}$ In addition, people with SCD tend to have more significant and prolonged hyphema, as well as an increased risk for secondary hemorrhage. ${ }^{274}$ Aggressive treatment such as anterior chamber paracentesis or surgical evacuation of a clot may be vision sparing in people with SCD with sustained elevated IOPs that are not responsive to medical management. ${ }^{271,273-275}$

CRAO is a rare cause of acute blindness reported almost exclusively in children and young adults with SCA. ${ }^{276}$ It results from thrombus formation in the artery. CRAO causes infarction of the inner retina ${ }^{277}$ and results in macular ischemia and potential macular infarction. People typically present with sudden, painless unilateral or 
bilateral loss of vision. CRAO has been observed in people with SCD in association with increased IOP secondary to hyphema, ${ }^{276}$ moyamoya syndrome, ${ }^{278}$ or ACS. ${ }^{279}$ CRAO can also occur spontaneously. ${ }^{280-282}$

Orbital infarction is another rare but serious complication of SCD, typically occurring during a VOC. This infarction of the orbital bones is often complicated by hematomas, thought to be a result of ischemic vessel wall necrosis. Because space in the orbital cavity is limited, the inflammatory response generated by infarcted bone may result in further compromise of important eye structures. People typically present with protrusion of the eye, eye pain, and lid and/or orbital edema. On examination, people will have decreased visual acuity and extraocular motility. Differential diagnosis includes periorbital infection due to orbital cellulitis, orbital abscesses, or osteomyelitis, and OCS. Radiographic imaging aids in diagnosis. ${ }^{283-285}$ In the case of periorbital infection or orbital bone infarction, rapidly progressive symptoms despite maximal medical management may require surgical intervention.

OCS, also known as orbital apex syndrome, is defined by the presence of compressive optic neuropathy and markedly decreased extraocular motility secondary to involvement of the branches of cranial nerves III and V. Recently, OCS has been described as a result of orbital inflammation after sphenoid bone infarction with subperiosteal hematomas, ${ }^{285}$ suggesting significant overlap between orbital infarction and OCS. Prompt initiation of corticosteroids once infection is ruled out can result in reversal of OCS. ${ }^{285}$ Diagnostic imaging includes MRI. Surgical intervention may be needed if medical management fails to resolve the compressive optic neuropathy.

\section{Key Question}

\section{KQ17. In people with SCD and acute eye symptoms, what is the optimal management strategy to preserve vision and prevent long-term ocular complications?}

\section{Summary of the Evidence}

Six studies (three RCTs and three observational studies) and 29 case reports described sickle cell-related acute or chronic ocular complications. Of these, the RCTs and the observational studies assessed the management of chronic sickle cell retinopathy, which is discussed in the "Managing Chronic Complications of Sickle Cell Disease" chapter. Twenty-two of the 29 case reports addressed acute complications alone (see evidence tables). Very little data exist to evaluate the most effective therapy to preserve vision during and after acute eye emergencies. The evidence that does exist comes from the case reports, which describe various and often multiple interventions (e.g., calcium channel blockers, intravenous hydration, surgical interventions) for the treatment of hyphema, CRAO, orbital infarction, and OCS. There was not enough evidence to make a recommendation about using transfusion to manage these acute complications.

Due to the paucity of available data, in developing recommendations for acute ocular conditions, the panel placed a high value on the outcome of vision preservation and less value on the burdens and harms of interventions supported with lower quality evidence. 


\section{Recommendations}

1. Immediately examine for hyphema anyone with SCD who presents with eye trauma. If hyphema is present, immediately refer to an ophthalmologist for further management.

(Consensus-Panel Expertise)

2. Promptly refer anyone with SCD exhibiting signs and symptoms such as protrusion of the eye, changes in visual acuity (flashers or floaters), and unilateral or bilateral loss of vision to an eye specialist capable of performing a dilated eye exam to assess visual acuity, intraocular pressure, and the peripheral retina.

(Consensus-Panel Expertise)

3. Manage acute ocular complications in consultation with an ophthalmologist, hematologist, and other specialists with expertise in SCD.

(Consensus-Panel Expertise) 



\section{Chapter 4: Managing Chronic Complications of Sickle Cell Disease}

\section{Introduction}

Complications may occur early and span the entire life of individuals affected by SCD. Direct SCD complications may include acute or chronic pain syndromes, significant anemia and its sequelae, as well as organ damage and failure. Other coexisting complications may include rheumatoid arthritis and peptic ulcer disease. ${ }^{147,286}$ Common acute complications and their sequelae are described in the "Managing Acute Complications of Sickle Cell Disease" chapter in these guidelines. This chapter focuses on the chronic complications of SCD. Chronic complications of SCD can affect almost any organ, and certain acute complications, such as stroke and priapism, often evolve into chronic phases that require special approaches to management.

The phenotypic expression of chronic complications varies considerably among people, in the same person over time, and among the various subtypes of SCD. Because the incidence of chronic complications seems to increase with age, understanding their pathophysiology, precipitating factors, and predictors may help prevent or minimize long-term morbidity.

Just as the presentation and manifestation of chronic complications of SCD may vary, so have their definitions. Recently, a unified definition of each complication of SCD has been published, ${ }^{287}$ which may help stimulate further work to better describe and explain each complication. Without universal uniform definitions, the natural history of SCD complications and the effect of therapy will be difficult to determine.

In this chapter, recommendations related to the evaluation and management of the most common chronic complications of SCD are presented. For each complication discussed, information is presented on its frequency, most common presentations, usual evaluation, and treatment.

\section{Methodology}

Complete information about the methodology for these guidelines can be found in the "Introduction and Methodology" chapter (pages 1-9). The following information, specific to this chapter, supplements the standard methodology that was conducted for all clinical chapters of these guidelines.

A comprehensive study of several databases was conducted, and all human studies in English published from 1970 to July 2010 that addressed each PICOS question were identified. A total of 549 studies of complications were included. ${ }^{\text {h }}$ When the literature search found insufficient evidence on a topic (e.g., chronic pain management), these topics were supplemented with recommendations derived from other published guidelines by professional organizations which were based on systematic reviews of broader population groups; these recommendations are labeled "Consensus-Adapted." The key questions for this chapter can be found immediately before the Summary of the Evidence sections for the individual topics.

Sickle cell anemia (SCA) refers to the clinically similar disorders HbSS or HbS $\beta 0$-thalassemia. Sickle cell disease (SCD) refers to all disease genotypes, including SCA and compound heterozygous disorders, such as HbSC, HbSD, and HbS $\beta+$-thalassemia. The carrier state for hemoglobin S (HbAS or sickle cell trait) is not a form of SCD.

\footnotetext{
${ }^{\mathrm{h}}$ An updated search was performed to span the time from June 1, 2010 through April 2014. Five additional RCTs were identified, for a total of 549 studies, and a supplemental table reflecting these additions was added to the evidence table document.
} 
Detailed information on the evaluated studies as well as the observational and case studies/series referenced can be found in the Management of Sickle Cell Disease Complications evidence table available at http://www.nhlbi.nih.gov/guidelines/scd/index.htm.

\section{Chronic Pain}

\section{Background}

In SCD, pain is considered chronic if it lasts more than 3 months. People with SCD experience both nociceptive and neuropathic pain. Nociceptive pain is a hallmark of acute pain (see the "Managing Acute Complications of Sickle Cell Disease" chapter). Chronic pain, including that described in people without SCD, is often associated with neuropathic pain. The pathology of the transformation from chronic nociceptive pain to neuropathic pain is not well understood. The Pain in Sickle Cell Epidemiology Study (PiSCES) showed that adults reported chronic SCD pain at home during about 55 percent of the 31,017 days surveyed. ${ }^{145}$ Similarly, children reported SCD pain at home on about 9 percent of the 1,515 days surveyed. ${ }^{288}$ In the Multicenter Study of Hydroxyurea in Sickle Cell Anemia (MSH), at-home analgesics were used for SCD pain on 40 percent of diary days and during 80 percent of 2-week followup periods, with oxycodone and codeine being used most frequently. ${ }^{289}$

The major types of SCD-associated chronic pain include the following:

- Chronic pain often of unclear etiology. This type of chronic sickle cell pain may be an extension of recurrent acute painful episodes. Therefore, early and aggressive intervention in treating acute sickle cell pain may reduce the development of chronic pain.

- Chronic pain in a specific tissue or organ, such as avascular necrosis (AVN) of the hips, or leg ulcers. Chronic SCD pain is usually described as constant and deep, nagging, and achy in nature. It can occur in the chest, back, abdomen, extremities, neck, or head and is difficult to treat.

- Chronic neuropathic pain. This is usually described as burning, numb, tingling, lancinating, shooting, or paroxysmal in nature and is associated with a sensation of pins and needles. Its severity is also enhanced by exposure to either cold or heat. This pain can be secondary to either peripheral or central nerve injury or nerve dysfunction. SCD-related neuropathic pain has two etiologies. The first is tissue damage secondary to occlusion of blood vessels that supply the nerves as can be found in mental nerve neuropathy and spinal cord infarction. ${ }^{290-292}$ The second seems to be chronic pain. Persistent chronic pain, the resulting inflammation, and/or pain management seem to lead to neuropathic pain. ${ }^{286,293-296}$

- "Breakthrough" pain is another type of pain often identified by health care professionals who treat patients with SCD. This term literally means the act of breaking through pain relief. Originally used to describe patients with cancer pain who were maintained on a stable dose of analgesics, breakthrough pain was defined as a flair-up of sudden pain unresponsive to usual therapy. Such a flare-up is usually sudden and incidental, and can last from a few seconds to a few hours. There are currently no data that clearly describe or can be used to define breakthrough pain in SCD. ${ }^{297}$

The pathophysiology, management, and goals of treating chronic pain differ from those related to acute pain. Whereas the aim of acute pain treatment is to heal the acute process, the aim of chronic pain management is to restore function and improve the quality of life. With the onset of chronic pain of unknown etiology, there seems to be a process of "rewiring" in the brain, where the threshold for pain perception is lowered so that ambient environmental stimuli that are normally painless or mildly painful induce the perception of severe pain. ${ }^{298}$ Chronic pain is often associated with other conditions that enhance its chronicity. These include psychosocial factors such as depression, anxiety, feelings of despair, insomnia, loneliness, helplessness, posttraumatic stress disorder (PTSD), and dependence on pain medications. ${ }^{23,293,299}$ 
Management of chronic pain in people with SCD is a major challenge for health care professionals. The goals of providing adequate pain relief to improve functionality and quality of life must be balanced by the need to minimize the risk of abuse, misuse, or diversion of opioids - medications which are a mainstay in managing chronic pain in people with SCD. Believing the patient's report of pain is critical to optimizing therapeutic outcomes and achieving adequate pain relief and maintaining or improving functionality and the person's quality of life. $^{286}$

Medications used to treat SCD-related pain should be tailored to the individual. Medications include nonsteroidal anti-inflammatory drugs (NSAIDs), opioids, antidepressants, and anticonvulsant medications. Management of all types of chronic pain associated with SCD may be enhanced by adding nonpharmacologic approaches. These may include psychological intervention, occupational therapy, behavioral and cognitive interventions, acupuncture, mild to moderate exercise if tolerable, and aqua therapy.

\section{Key Question}

\section{KQ18. In people with SCD and chronic pain, what are the safest and most effective chronic pain management strategies and treatment algorithms (e.g., patient assessment and followup, use of chronic opioids, adjuvant pharmacological therapies, and behavioral therapies)?}

\section{Summary of the Evidence}

To develop recommendations for the management of chronic pain in SCD, the methodology team conducted a comprehensive systematic review of studies that evaluated the efficacy and harms of different management approaches for chronic pain in SCD. Eight studies (two RCTs and six observational studies) and 13 case reports were eligible for inclusion. ${ }^{294,296,300-305}$

One study explored general chronic sickle cell pain and compared utilization of massage therapy and progressive muscle relaxation to massage therapy alone and found no significant differences between the two approaches. ${ }^{301}$ The second study assessed hip pain and demonstrated a statistically significant difference between transcutaneous sodium salicylate iontophoresis and parenteral analgesics, favoring iontophoresis. ${ }^{300}$ The observational studies were fairly small and described various sickle cell-related pain presentations and management approaches. The baseline characteristics and outcomes of these studies are described in the evidence table. ${ }^{24,294,296,300-304,306}$

In general, the quality of the available evidence was very low, so the expert panel determined that higher quality evidence with better precision should be derived from studies that evaluated chronic pain management in other settings. Such a body of evidence is larger and includes a wider scope of interventions and comparisons, which could lead to more useful recommendations for practitioners caring for people with SCD who have chronic pain. The panel and the methodology team appraised the quality of the guidelines for the management of chronic pain published by the American Pain Society in collaboration with the American Academy of Pain Medicine. ${ }^{24}$ The quality of the guidelines was deemed acceptable, so the panel adapted selected recommendations applicable to people with SCD as shown below in the "Recommendations" section, and these are labeled accordingly. 


\section{Recommendations}

1. Determine the cause and type of SCD-related chronic pain. This includes chronic pain with objective signs such as avascular necrosis (AVN) and leg ulcers, and chronic pain without objective signs due to neuroplasticity of the peripheral or central nervous system.

(Consensus-Adapted)

2. Use a combination of the patient's response to treatment-including pain relief, side effects, and functional outcomes-to guide the long-term use of opioids.

\section{(Consensus-Adapted)}

3. Encourage people to use deep tissue/deep pressure massage therapy, muscle relaxation therapy, and self-hypnosis as indicated.

\section{(Weak Recommendation, Low-Quality Evidence)}

4. Use long- and short-acting opioids to manage chronic pain that is not relieved by nonopioids. (Consensus-Adapted)

5. Assess all people with SCD for chronic pain annually or more often as needed. This assessment should include descriptors of the pain; its severity on a numerical scale; its location; factors that precipitate or relieve it, including biopsychosocial factors; and its effect on the patient's mood, activity, employment, quality of life, and vital signs.

(Consensus-Adapted)

6. Use a partnership agreement leading to a written, individualized treatment plan (to include risks, benefits, and side effects) with the patient if long-term opioids are indicated. The partnership agreement should list the patient's rights and responsibilities, and the treatment plan should list the type, amount, and route of administration of the opioid in question, including random drug urine testing.

\section{(Consensus-Adapted)}

7. Appoint one physician or other clinician to write the biweekly to monthly prescriptions for long-term opioids. Refills without seeing the patient should be kept to a minimum, and people on chronic opioid therapy must be evaluated in person every 2-3 months.

(Consensus-Adapted)

8. Document all encounters with a patient, including medical history, physical exam, diagnosis, plan of management, type and amount of opioids prescribed and their side effects, if any, and lab data as needed.

\section{(Consensus-Adapted)}

9. Encourage people receiving opioids to increase their fluid intake, maintain dietary fiber intake per the current dietary fiber recommendations, and to use stool softeners and bowel stimulant laxatives such as senna and/or docusate as needed.

\section{(Consensus-Adapted)}

10. Believe the patient's report of pain and optimize therapeutic outcomes to achieve adequate pain relief and improve the patient's quality of life.

(Consensus-Adapted)

11. Refer patients for evaluation by a mental health professional such as a psychiatrist, social worker, or addiction specialist as needed.

(Consensus-Adapted)

12. Assess all people for other types of non-SCD related chronic pain including postoperative pain, pain due to trauma, pain due to therapy, iatrogenic pain, and pain due to comorbid conditions.

(Consensus-Adapted) 


\section{Avascular Necrosis}

\section{Background}

Avascular necrosis (AVN, also known as aseptic necrosis, osteonecrosis, or ischemic necrosis) is bone death due to compromised blood supply. Necrosis can occur when capillaries are occluded by sickled erythrocytes at distal portions of a bone near a joint where hypoxia is maximal and collateral circulation is inadequate. ${ }^{307}$ The hip joint is the most common site of AVN. Involvement of the shoulder and other joints is less common. Risk factors for AVN of the femoral head include SCD genotype, age, frequency of painful episodes, hemoglobin level, and $\alpha$-gene deletion. The overall prevalence of AVN in SCD is about 10 percent, whereas in people with hemoglobin SS, it is about 50 percent by age $33 .^{308,309}$ People with HbSS and concomitant $\alpha$-thalassemia are at particular risk. $^{308,309}$ The SCD genotypes that are associated with relatively mild anemia, such as HbSS- $\alpha$-thalassemia and $\mathrm{HbS} \beta^{0}$-thalassemia, are at a particularly high risk to develop AVN at a younger age. ${ }^{308,309}$

AVN of the femoral head causes chronic severe pain and disability. The pain is generally worse on walking, relieved by rest, and may be accompanied by a moderate or severe limitation of motion when the patient bears weight on the affected extremity. About 40-80 percent of cases of AVN of the hips are bilateral and, hence, evaluation of patients with AVN should focus on both hips. ${ }^{310}$

The therapeutic approach to AVN depends on the stage of the disease. Ficat ${ }^{311}$ proposed a four-stage radiographic classification of AVN of the hip based on plain radiography. MRI was not available at the time. Steinberg et al. ${ }^{312}$ expanded the Ficat staging system into six stages using MRI data. ${ }^{312,313}$ A report from the Comprehensive Sickle Cell Centers (CSCC) investigators defined an adaptation ${ }^{287}$ from the Ficat and Steinberg systems that combines radiography, MRI, and bone scans. The adaptation is provided below in exhibit 8 .

\section{Exhibit 8. Stages of Avascular Necrosis}

\begin{tabular}{|c|c|}
\hline Stage & Radiographic Signs \\
\hline EARLY: Stage 0. Preclinical & - None; marrow necrosis may be present histologically \\
\hline EARLY: Stage I. Preradiographic & - None; abnormal MRI with marrow and bone necrosis \\
\hline $\begin{array}{l}\text { EARLY: Stage II. Before flattening of head or sequestrum } \\
\text { formation }\end{array}$ & - Diffuse porosis, sclerosis, or cysts \\
\hline TRANSITION & $\begin{array}{l}\text { - Femoral head flattening } \\
\text { - Crescent sign }\end{array}$ \\
\hline LATE: Stage III. Collapse & $\begin{array}{l}\text { - } \text { Broken contour of head } \\
\text { - Sequestrum } \\
\text { - Joint space normal }\end{array}$ \\
\hline LATE: Stage IV. Osteoarthritis & $\begin{array}{l}\text { - Flattened contour } \\
\text { - Decreased joint space } \\
\text { - } \quad \text { Collapse of head }\end{array}$ \\
\hline
\end{tabular}

Most orthopedists consider core decompression to be most beneficial for Ficat stage I and II of AVN of the hip. $^{310,314,315}$ 


\section{Key Question}

\section{KQ19. In people with SCD and AVN, what are the most effective management strategies to reduce pain and functional disability (e.g., analgesics, physical therapy, surgery, or transfusion therapy)?}

\section{Summary of the Evidence}

The literature review yielded 1 RCT, 16 observational studies, and 16 case reports describing AVN and treatment outcomes. The overall quality of the evidence was low.

The $\mathrm{RCT}^{316}$ was a randomized prospective multicenter study of 38 adults ( 81 percent of enrollees), which evaluated the safety of hip core decompression and compared the results of decompression and physical therapy with those of physical therapy alone for the treatment of osteonecrosis of the femoral head in people with all types of SCD. Results showed that physical therapy alone was as effective as hip core decompression followed by physical therapy in improving hip function. However, the evidence provided by this study is limited due to its small sample size and the high attrition rate.

The 16 observational studies and 16 case reports described AVN of various bones in the context of SCD. These studies included more than 350 people (mostly adults) and most commonly reported on people with SCD with AVN of the femoral head. All studies but one ${ }^{317}$ were noncomparative, used hip arthroplasty, and reported a high success rate. A few studies ${ }^{318,319}$ reported the use of standard symptomatic therapy with minimal success. In the comparative study, ${ }^{317}$ the benefit of the surgical intervention (core decompression) in improving pain and evolution of necrotic lesions was significant relative to conservative management.

The methodological quality of the 16 observational studies was low (mainly observational noncontrolled studies with unclear enrollment criteria). The single comparative study had groups with similar baseline characteristics and outcome ascertainment methods. None of the studies reported adjustment of analyses for confounders.

\section{Recommendations}

1. Evaluate all children and adults with $S C D$ and intermittent or chronic hip pain for AVN by history, physical exam, radiography, and MRI as needed.

(Strong Recommendation, Low-Quality Evidence)

2. Treat AVN with analgesics and consult physical therapy and orthopedics for assessment and followup. (Strong Recommendation, High-Quality Evidence)

3. Refer symptomatic patients with advanced stages of $A V N$ to an orthopedic surgeon and SCD specialist for evaluation and possible hip arthroplasty.

(Consensus-Panel Expertise)

\section{Leg Ulcers}

\section{Background}

Leg ulcers are a common complication of SCD in general and SCA in particular. Leg ulceration was reported in all of the first four people with SCD described in the English literature. ${ }^{320}$ Data from the Cooperative Study of Sickle Cell Disease (CSSCD) in the United States ${ }^{321}$ found active leg ulcers at entry in 2.5 percent of 2,075 
people aged 10 years or older and in none of 1,700 people less than 10 years old. Among those with active leg ulcers, about 22 percent were between the ages of 10 and 20 .

Data on leg ulcers from the $\operatorname{CSSCD}^{321}$ identified five factors which could affect the person's risk. Leg ulcers were more common in males and older people and less common in people with $\alpha$-gene deletion, high total $\mathrm{Hb}$ level, and high levels of $\mathrm{HbF}^{322,323}$ Trauma, infection, and severe anemia may predispose people to ulcer formation. Studies showing a positive association between leg ulcers and the severity of hemolysis and priapism are disputed. ${ }^{324-326}$ The ulcers occur most frequently on the medial or lateral surfaces of the ankles. Leg ulcers can range from mild and small to large and severe. Severity can be based on depth and duration. Osteomyelitis may complicate chronic leg ulcers, especially deeper ones. A bone scan or MRI and bone biopsy are used to assess this complication. Multidisciplinary teams including wound care specialists have been developed to provide support and consultation in the management of recurrent and recalcitrant leg ulcers.

\section{Key Question}

KQ20. In people with SCD and leg ulcers, what are the most effective therapies to accelerate ulcer healing (e.g., topical therapy, surgery, or antibiotics)?

\section{Summary of the Evidence}

Five RCTs, three observational studies, and a case series described various approaches to manage leg ulcers in people with SCD and evaluated topical and systemic agents. The methodological quality of the studies was fair, but the studies had small sample size, which led to imprecise estimates of treatment effect and weak inference. The overall quality of the supporting evidence was low to moderate.

The five RCTs included a total of 155 people and had followup periods of 8 weeks to 6 months. Four studies ${ }^{327-}$ ${ }^{330}$ compared different topical modalities, including arginylglycylaspartic acid (RGD) peptide; arginine butyrate; DuoDerm; solcoseryl; and an aerosolized preparation of neomycin, bacitracin, and polymyxin B to either standard care or placebo. One study ${ }^{331}$ compared oral propionyl-L-carnitine to placebo. Propionyl-L-carnitine was not shown to have any significant differences in healing effect. Of the topical preparations, RGD peptide and the arginine butyrate/standard care combination showed a significant improvement in healing rates. The aerosol solution trial showed significant reduction in ulcer size for ulcers with a positive bacterial swab test. The studies also found severe intolerance to DuoDerm and good tolerance to solcoseryl without any significant differences in healing rates.

The three observational studies ${ }^{332-334}$ enrolled more than 70 people and reported no difference in healing between natural honey and eusol dressing (sodium hypochlorite disinfectant); higher healing rate with oral zinc sulphate compared to placebo; and favorable results with hydrocolloid dressing (DuoDerm). The case series ${ }^{335}$ reported improved healing after 6 weeks of treatment with subcutaneous heparin and human antithrombin concentrate. The quality of evidence of these observational data is low, thus limiting the ability to make inferences applicable to the general population. 
1. Inspect the lower extremities during physical examination for active or healed ulcers, record their number, and measure their depth.

(Weak Recommendation, Low-Quality Evidence)

2. Treat leg ulcers in patients with SCD with initial standard therapy (i.e., debridement, wet to dry dressings, and topical agents).

(Moderate Recommendation, Low-Quality Evidence)

3. Evaluate people with chronic recalcitrant deep leg ulcers for osteomyelitis.

(Moderate Recommendation, Low-Quality Evidence)

4. Evaluate possible etiologies of leg ulcers to include venous insufficiency and perform wound culture if infection is suspected or if the ulcers deteriorate.

(Moderate Recommendation, Low-Quality Evidence)

5. Treat with systemic or local antibiotics if leg ulcer site is suspicious for infection and wound culture is positive and organism susceptible.

(Moderate Recommendation, Low-Quality Evidence)

6. Consult or refer to a wound care specialist or multidisciplinary wound team for persistent or recalcitrant leg ulcers. (Consensus-Panel Expertise)

\section{Pulmonary Hypertension}

\section{Background}

Pulmonary hypertension (PH) is defined as an elevation of the resting mean pulmonary arterial pressure ( $\geq 25$ $\mathrm{mmHg}$ ) as determined by right heart catheterization (RHC). ${ }^{43}$ There are several potential etiologies for elevation in mean pulmonary artery pressure in people with SCD. Chronic hemolytic anemias, including SCD, may result in pulmonary vascular changes leading to pulmonary arterial hypertension (PAH), and are placed in Group 1 of the current classification (https://www.nhlbi.nih.gov/health/health-topics/topics/pah/types.html). ${ }^{44}$ This type of pulmonary hypertension may occur in up to 10 percent of those with SCA and accounts for 40 to 50 percent of cases of $\mathrm{PH}{ }^{45,46,336}$ The second most common type of PH in SCD is pulmonary venous hypertension $(\mathrm{PVH})$, assigned to Group 2 in the current classification, which is associated with an elevated pulmonary capillary wedge pressure of $\geq 15 \mathrm{mmHg}$. ${ }^{43-47}$ This is often associated with left ventricular diastolic dysfunction. ${ }^{48}$ $\mathrm{PH}$ also occurs in the setting of chronic lung disease, chronic thromboembolic disease, or can be due to unclear or multiple mechanisms (Groups 3, 4, and 5 of the classification, respectively). Because these circumstances may also be present in individuals with SCD, a thorough evaluation of mechanisms and comorbidities should be undertaken if $\mathrm{PH}$ is found.

Initial testing for $\mathrm{PH}$ has been done with an echocardiography assessment to estimate pulmonary artery pressure using tricuspid regurgitant jet velocity (TRV) ${ }^{43,45-47}$ but diagnosis requires right heart catheterization and direct measurement of the pulmonary arterial pressure and vaso-reactivity of the vessels. ${ }^{43,45,50,56,337}$ Transient elevation in TRV has been observed during acute vaso-occlusive episodes in individuals with $\mathrm{SCD},{ }^{51}$ which may not reflect baseline values or present chronic $\mathrm{PH}$.

The main symptoms of $\mathrm{PH}$ include shortness of breath during routine activity, such as climbing two flights of stairs; fatigue; lethargy; chest pain; palpitations; syncope; peripheral edema; and decreased appetite. ${ }^{49}$ Careful history taking is needed to distinguish symptoms related to the anemia of SCD itself from the new onset of symptoms related to the development of $\mathrm{PH}$. 
Observational studies show an increase in all-cause mortality for adults with SCA with an elevated TRV by echocardiography, ${ }^{47,57,61,338}$ although this association has not been found in children. In children and young adults with relatively normal renal function, only 25-30 percent of those with an elevated TRV may have an elevated pulmonary artery pressure measured by right heart catheterization. ${ }^{46}$ Older adults with SCA and a high TRV are more likely to have an elevated pulmonary pressure, although 40 percent of those with a high TRV will have an elevated wedge pressure suggesting left heart disease. A commonly associated finding is renal insufficiency. ${ }^{47,57,58}$ The Treatment of Pulmonary Hypertension and Sickle Cell Disease with Sildenafil Therapy (walk-PHaSST) study was published outside of the range of the evidence review for these guidelines and thus was not included. This study enrolled 33 subjects with SCD and PH defined by an elevated TRV of $\geq 2.7 \mathrm{~m} / \mathrm{sec}$ and a 6-minute walk distance (6MWD) of 150-500 meters. ${ }^{47}$ RHC was required in the TRV $\geq 3.0 \mathrm{~m} / \mathrm{sec}$ group; all subjects were randomized to sildenafil or placebo regardless of findings at RHC. This study was closed early due to an increase in serious adverse events associated with sildenafil use; estimation of results by futility analysis suggested no improvement in 6MWD would be demonstrated if the study continued. These data confirm earlier data that RHC is necessary to confirm the presence of PH and distinguish the mechanism of disease before considering therapy for PH. It is unknown if intervention for SCD (e.g., transfusion or hydroxyurea) would change the all-cause mortality associated with an elevated TRV.

\section{Key Question}

KQ21. In people with SCD and PH, what are the most effective therapies to reduce mortality (e.g., transfusion, hydroxyurea, and other pharmacological agents)?

\section{Summary of the Evidence}

Two RCTs, seven observational studies, and three case reports examined the management of PAH. No clear therapeutic benefit has been shown for any pharmacotherapy for PAH in people with all types of SCD, and the overall quality of the evidence on therapy was considered very low.

The two RCTs were reported in one paper, and enrolled 26 people, blinded patients and outcome assessors to the intervention assignment, and did not report any baseline imbalances or allocation concealment. ${ }^{339}$ Both trials were stopped prematurely due to slow enrollment. The trials compared bosentan to placebo and showed no improvement in the 6MWD or levels of pulmonary hypertension.

The seven observational studies included more than 200 people and evaluated various aspects of PAH. Five studies examined various therapies, and two looked at mortality rates. Increased mortality was reported in all people with $\mathrm{SCD}^{339-343}$ with true PAH (55 percent vs. 21 percent 10-year mortality respectively in all people with SCD with and without PAH). ${ }^{61,338}$ The five small observational studies reported various levels of benefit from five different types of pharmacotherapy, but no consistent definition of PAH was used across these uncontrolled studies, making it difficult to compare results. The five therapies studied were bosentan, sildenafil, L-arginine, L-carnitine, and hydroxyurea. Both bosentan and sildenafil were reported to increase 6MWD. ${ }^{339,340}$ L-arginine was reported to improve pulmonary arterial function, although this was a short-term benefit. Although results from a pilot study of sildenafil suggested improved exercise capacity in pulmonary hypertension, the study was stopped early due to safety concerns and the authors cautioned that additional studies on the safety of sildenafil in this patient population were needed. ${ }^{341}$ L-carnitine was reported to improve cardiac diastolic function, ${ }^{342}$ and hydroxyurea was reported to normalize elevated tricuspid regurgitant velocity (TRV), but this was not sustained long term. ${ }^{343}$ 
1. If people with SCD have symptoms or signs suggestive of $\mathrm{PH}$, refer them for echocardiography. (Strong Recommendation; Moderate-Quality Evidence)

2. For people with an elevated TRV $\geq 2.5 \mathrm{~m} / \mathrm{sec}$ by echocardiography, consult a provider with expertise in pulmonary hypertension to guide further assessment and management, including right heart catheterization, and consideration of $\mathrm{PH}$ therapy.

(Consensus-Panel Expertise)

\section{Renal Complications}

\section{Background}

Chronic kidney disease (CKD) is defined as either having a glomerular filtration rate (GFR) of

$<60 \mathrm{~mL} / \mathrm{min} / 1.73 \mathrm{~mL}$ for $\geq 3$ months with or without kidney damage or having evidence of kidney damage for $\geq 3$ months, with or without decreased GFR. Evidence of kidney damage includes pathologic abnormalities or markers of kidney damage (i.e., proteinuria) independent of cause. Kidney disease severity is classified into five stages according to the level of GFR (see exhibit 9 below). ${ }^{344}$

\section{Exhibit 9. Stages of Kidney Disease by GFR Levels}

\begin{tabular}{|l|l|}
\multicolumn{1}{c|}{ Stage } & \multicolumn{1}{c|}{ GFR Parameters } \\
\hline Stage 1 & - $\quad$ Kidney damage with normal or increased GFR $\left(\geq 90 \mathrm{~mL} / \mathrm{min} / 1.73 \mathrm{~m}^{2}\right)$ \\
\hline Stage 2 & - $\quad$ Kidney damage with mildly decreased GFR $\left(60-89 \mathrm{~mL} / \mathrm{min} / 1.73 \mathrm{~m}^{2}\right)$ \\
\hline Stage 3 & - $\quad$ Moderately decreased GFR $\left(30-59 \mathrm{~mL} / \mathrm{min} / 1.73 \mathrm{~m}^{2}\right)$ \\
\hline Stage 4 & - Severely decreased GFR $\left(15-29 \mathrm{~mL} / \mathrm{min} / 1.73 \mathrm{~m}^{2}\right)$ \\
\hline Stage 5 & - $\quad$ Kidney failure (ESRD); GFR $<15 \mathrm{~mL} / \mathrm{min} / 1.73 \mathrm{~m}^{2}$ or on dialysis \\
\hline
\end{tabular}

Source: Levey AS, Eckardt KU, Tsukamoto Y, Levin A, Coresh J, Rossert J, De Zeeuw D, Hostetter TH, Lameire N, Eknoyan G. Definition and classification of chronic kidney disease: a position statement from Kidney Disease: Improving Global Outcomes (KDIGO). Kidney Int. 2005 Jun;67(6):2089-100. ${ }^{344}$

An estimated 23 million Americans have CKD including 4-18 percent of people with SCD. ${ }^{345}$ In one study, renal failure was seen in 4.2 percent of people with SCA. ${ }^{346}$ In this study, 68 percent of people had proteinuria (defined as any abnormal urinary protein), 40 percent had nephrotic syndrome, and 33 percent had hypertension (HTN) prior to developing renal failure. ${ }^{346}$ In another study, Falk et al. ${ }^{347}$ evaluated all people with SCD, including both children and adults, followed at the University of North Carolina and Duke University; 26 percent had proteinuria on urine dipstick. Finally, in a study of 300 adults with SCD, the prevalence of any albuminuria in people with SCA was 68 percent, and the prevalence in other genotypes was 32 percent. $^{348}$

Identification of early renal disease in people with SCD is important, as these individuals hypersecrete creatinine through the proximal tubules, thus masking significant renal impairment before the serum creatinine rises. ${ }^{345}$ Microalbuminuria is defined as urinary albumin excretion greater than $30 \mathrm{mg}$ albumin per gram urine creatinine in two of three spot urine specimens or 30-299 mg albumin in 24-hour urine collection. Macroalbuminuria (proteinuria) is defined as urinary albumin excretion of 300-3,500 mg albumin in 24-hour urine collection. ${ }^{344}$ Microalbuminuria is most often the first manifestation of CKD in SCD. One study showed a prevalence of 16 
percent in affected children, ${ }^{349}$ and another study showed a prevalence of 32.9 percent in adults with SCD. ${ }^{350}$ Spot urine protein/creatinine ratio has not been validated in SCD because creatinine is hyperexcreted.

The most common renal complication in people with SCD is hyposthenuria, or the inability to concentrate the urine, which is progressive with age. ${ }^{351}$ This is due to the loss of deep juxtamedullary nephrons. Frequent urination is common in people with SCD and is usually due to hyposthenuria. Because of their hyposthenuria, individuals with SCD are also at higher risk for intravascular volume depletion, as they cannot respond to decreased oral fluid intake by concentrating their urine. In addition, hyposthenuria also causes enuresis, which is prevalent among individuals with SCA, with up to 42 percent of children ages 6 to 8 and 9 percent of adults ages 18 to 20 experiencing this complication. ${ }^{352}$

Renal papillary necrosis, which often causes hematuria, is thought to be due to medullary infarction from obstruction of the vessels supplying the vasa recta. The prevalence of renal papillary necrosis was found to be as high as 23 percent in asymptomatic people with SCA undergoing urography. ${ }^{353}$ Proteinuria due to glomerular injury is also common, but both microalbuminuria and macroalbuminuria are typically asymptomatic. Other early manifestations that should lead providers to investigate people for renal disease include HTN and gout. Joint pain due to gout can often be mistaken for vaso-occlusive episode pain. Diagnosis and management of gout in individuals with SCD is the same as in other populations.

There have not been any studies looking at the utility of renal biopsy in individuals with SCD. One study that examined 18 renal biopsy specimens found four histopathologic variants: focal segmental glomerulosclerosis (FSGS) (39 percent), membranoproliferative glomerulonephritis (28 percent), thrombotic microantigopathy glomerulopathy (17 percent), and specific sickle cell disease glomerulopathy (17 percent). ${ }^{354}$ The authors of this study note that the long-term outcomes were not different according to the histologic lesions that were identified, with 50 percent of cases having chronic renal failure after a mean followup of 28 months. The decision to perform renal biopsy should be individualized for each patient.

\section{Key Question}

KQ22. In people with SCD and CKD, what are the interventions (including pharmacotherapy, dialysis, and renal transplant) that slow the deterioration of renal function, prevent the development of end-stage renal disease, and reduce mortality?

\section{Summary of the Evidence}

One RCT, 5 observational studies, and 10 case reports examined the management of several acute and chronic renal complications of SCD. Although numerous SCD-related renal abnormalities have been described in the literature (e.g., hyposthenuria, hematuria, impaired urinary potassium excretion and acidification, tubular and glomerular dysfunction, infection, medullary carcinoma, and acute necrosis and renal failure), most were without effective therapeutic approaches or clear prognosis. The overall quality of the evidence was low.

A double-blind, placebo-controlled randomized trial of 22 normotensive adults with SCA and persistent microalbuminuria found that captopril $\left(25 \mathrm{mg} /\right.$ day) for 6 months significantly reduced albuminuria. ${ }^{75}$

One observational study included more than 300 individuals with SCD and evaluated them for renal dysfunction. $^{347}$ Ten people were found to have proteinuria (urinary protein, $\geq 0.5 \mathrm{~g}$ per day) and serum creatinine concentrations of $<2.0 \mathrm{mg} / \mathrm{dL}$. They underwent treatment with enalapril for 2 weeks and had a decrease in proteinuria with a mean decrement of 57 percent below baseline. An observational study of 191 patients with SS 
with a mean followup of 2.19 years demonstrated that microalbumin excretion normalized in 44 percent of patients treated with hydroxyurea and 56 percent of patients treated with ACEI. ${ }^{355}$

One observational study looked at the prevalence of microalbuminuria in children and found it in 19 of 120 children with $\mathrm{SCD} .{ }^{40}$

Two observational studies enrolled 91 people and evaluated the role of renal transplant in end-stage renal disease. The larger study was a retrospective study comparing patient and renal allograft outcomes for individuals with SCD $(n=82)$ compared to those without $\operatorname{SCD}(n=22,565)$ who were transplanted and compared to those with SCD who did not undergo transplant. ${ }^{170}$ The study reported incidence rates of 26 percent and 24 percent for delayed predischarge and acute graft rejection, respectively. There was a trend towards improved survival in the transplant group compared to waitlisted individuals. The second smaller study reported a survival rate of 89 percent in the recipient of the graft, but the study did not have a comparison arm. ${ }^{171}$

\section{Recommendations}

1. If microalbuminuria or macroalbuminuria is identified, order a 24-hour urine test for protein.

(Consensus-Panel Expertise)

2. Refer people with proteinuria ( $>300 \mathrm{mg} / 24$ hours) to a nephrologist for further evaluation. (Strong Recommendation, Low-Quality Evidence)

3. For adults with microalbuminuria without other apparent cause, initiate ACE inhibitor therapy. (Moderate Recommendation, Moderate-Quality Evidence)

4. For adults with proteinuria without other apparent cause, initiate ACE inhibitor therapy. (Moderate Recommendation, Low-Quality Evidence)

5. For children with microalbuminuria or proteinuria, consult a nephrologist. (Consensus-Panel Expertise)

6. Consider patients with SCD with modest elevations of serum creatinine $(>0.7 \mathrm{mg} / \mathrm{dL}$ in children, $>1.0 \mathrm{mg} / \mathrm{dL}$ in adults) to have renal impairment and refer to a nephrologist for further evaluation.

(Consensus-Panel Expertise)

7. Give ACE inhibitor therapy for renal complications when indicated even in the presence of normal blood pressure. (Moderate Recommendation, Low-Quality Evidence)

8. Renal replacement therapy (e.g. hemodialysis, peritoneal dialysis, and renal transplantation) should be used in people with SCD if needed.

(Strong Recommendation, Low-Quality Evidence)

\section{Stuttering/Recurrent Priapism}

\section{Background}

Stuttering priapism is the occurrence of multiple self-limited episodes of unwanted, often painful erections lasting $<4$ hours. ${ }^{172}$ Priapism, including stuttering priapism, is common, affecting 35 percent of boys and men with SCD. ${ }^{173}$ Stuttering priapism may lead to a major episode of greater than 4 hours' duration and may adversely affect quality of life and lead to impotence. ${ }^{172,173}$ Treatment with chronic hormonal therapy, transfusion therapy, and other treatments may reduce or eliminate these episodes; however, there are no data demonstrating improvement in functional outcomes. Therefore, the decision to treat must be balanced against the side effects of interventions, which can include decreased libido. 


\section{Key Question}

KQ23. In people with SCD and stuttering priapism, what is the relative efficacy of the available treatments (chronic hormonal therapy, chronic transfusion therapy, alpha-adrenergic agents, PDE-5 esterase inhibitors, and hydroxyurea) on recurrence of priapism and sexual functional outcomes?

\section{Summary of the Evidence}

One RCT, 7 observational studies, and 39 case reports described priapism in the setting of SCD. Of these, only two studies evaluated the chronic management of priapism: the RCT and one observational study. Both studies were small, thus making the overall quality of the evidence very low.

The RCT noted cessation of bouts of priapism with stilbestrol during a 2-week cross-over phase ${ }^{174}$ in nine men with SCD. The observational study involved 35 participants and examined the effects of finasteride on recurrences of priapism. ${ }^{175}$ It reported a decrease in the number of priapic episodes and increased length of time between episodes.

There are no data demonstrating improvement in functional outcomes, so the potential benefits must be balanced against the side effects of interventions, including decreased sexual function. However, even in the absence of RCTs demonstrating long-term benefit, individualized therapy devised in consultation with a urologist may be considered for symptomatic relief.

\section{Recommendations}

1. In men and boys with SCD and recurrent or stuttering priapism, offer evaluation and treatment in consultation with a sickle cell disease specialist and a urologist, especially when episodes increase in severity or frequency.

(Weak Recommendation, Low-Quality Evidence)

\section{Ophthalmologic Complications}

\section{Background}

Chronic ophthalmological complications of SCD include proliferative sickle retinopathy (PSR) and vitreous hemorrhage. They occur in up to 50 percent of individuals with SCD, and are found more frequently in persons with $\mathrm{HbSC}$ disease and HbSS. ${ }^{356,357}$ The presence of PSR is associated with significant visual loss, ${ }^{83}$ and its peak prevalence in $\mathrm{HbSC}$ disease occurs earlier than in HbSS (i.e., about ages 15 to 24 in men and ages 20 to 39 in women). ${ }^{358}$

Ischemia due to vaso-occlusion of retinal arterioles causes the release of vascular tissue factors that stimulate angiogenesis. The neovascular tissue is predisposed to hemorrhage and vitreoretinal traction forces. Although these preretinal neovascular formations are bright red when viable, they appear white following auto-infarction, when they resemble and are called "sea fans."

PSR is characterized by five stages, ${ }^{359}$ beginning with peripheral arterial occlusion and vascular remodeling (Stages I-II), subsequent neovascularization and sea fan formation (Stage III), and ultimately vitreous hemorrhage (Stage IV) and retinal detachment (Stage V) (exhibit 10). All can be detected by using direct and indirect ophthalmoscopy, slit lamp biomicroscopy, and fluorescein angiography. 


\section{Exhibit 10. Stages of Proliferative Sickle Retinopathy (PSR)}

\begin{tabular}{|c|c|}
\hline Stages of PSR & Retinal Characteristics \\
\hline Stages I-II & - Peripheral arterial occlusion and vascular remodeling \\
\hline Stage III & - Neovascularization and sea fan formation \\
\hline Stage IV & - Vitreous hemorrhage \\
\hline Stage V & - Retinal detachment \\
\hline
\end{tabular}

Stages IV and V appear to be more common in individuals with HbSC disease.

Vitreous hemorrhage is a severe complication of $\mathrm{PSR}^{360}$ caused by mechanical stress from trauma or by normal vitreous movement on the delicate neovascular formation growing from the retina into the vitreous chamber.

Spontaneous regression of PSR may occur in about 32 percent of all affected eyes, and lack of progression of sea fans may occur in some people. ${ }^{84}$ PSR is commonly managed with laser photocoagulation after consultation with an ophthalmologist. Surgical intervention, including vitrectomy to treat severe vitreous hemorrhage, may be indicated in some people.

\section{Key Question}

KQ24. In people with SCD and chronic ophthalmic complications (proliferative sickle retinopathy or vitreous hemorrhage), what are the most effective management strategies (surgery, laser therapy, or conservative management) to improve and preserve vision?

\section{Summary of the Evidence}

Six studies (three RCTs, three observational) and 28 case reports described sickle cell-related acute or chronic ocular complications. The overall quality of evidence for laser photocoagulation was considered high, while the evidence for surgery in people refractory to medical management was considered low.

The three RCTs included 248 people (with likely overlapping populations, the majority of whom were adults) and assessed PSR and the benefit of laser photocoagulation compared to observation. ${ }^{86,87,361}$ One study reported more than a 50 percent decrease in the rates of loss of visual acuity, and another found that laser photocoagulation was helpful in inducing lesion regression but only in people younger than 25 years of age. Two of the RCTs reported a significant decrease in the incidence of vitreous hemorrhage, from 45 percent to 4 percent. None of the trials had any form of masking, allocation concealment, or differences in baseline characteristics of the participants.

The three observational studies included more than 140 people, mostly adults, and assessed the roles of laser photocoagulation and surgery in treating sickle cell-related retinopathies. ${ }^{362-364}$ One study found improvement in 83 percent of eyes that received surgery (pars plana vitrectomy) compared to 20 percent spontaneous improvement in the observation arm. One uncontrolled study found lesion regression in 79 percent of treated eyes, with vitreous hemorrhage occurring in only one patient. The last study found benefit from photocoagulation only in "class B" retinopathy (elevated sea fan with hemorrhage). Complications occurred in 13 percent of the untreated people, but not in any treated ones. 


\section{Recommendations}

1. Refer persons of all ages with PSR to an ophthalmologist for evaluation and possible laser photocoagulation therapy. (Strong Recommendation, Moderate-Quality Evidence)

2. Refer children and adults with vitreoretinal complications of PSR refractory to medical treatment for evaluation and possible vitrectomy.

(Strong Recommendation, Low-Quality Evidence) 



\section{Chapter 5: Hydroxyurea Therapy in the Management of Sickle Cell Disease}

\section{Introduction}

This chapter addresses the use of hydroxyurea (also called hydroxycarbamide) in adults and children who have SCD. Hydroxyurea can reduce the frequency of sickle cell-related pain and the incidence of acute chest syndrome (ACS). A brief overview of these complications will be presented.

Pain is the most common symptom of SCD. Pain can be acute, chronic, or an acute episode superimposed on chronic pain. Smith and colleagues ${ }^{145}$ collected daily pain diaries for 232 adults with SCD; pain was reported on 54.5 percent of the more than 30,000 days analyzed. However, people sought medical care for pain on only 3.5 percent of those days. These data suggest that many people who have SCD may be undertreated for their pain, may not perceive a benefit of treatment, or may have learned to self-manage their pain. Understanding of the processes that lead to an acute vaso-occlusive pain crisis and the pathophysiology of the chronic pain syndrome remains limited. It is known that rigid red blood cells (RBCs) obstruct the microvasculature; however, a full understanding of how these events start and what role other factors play in this process - such as vascular adhesion molecules, leukocytes, reticulocytes, endothelial cells, and platelets - has not been fully elucidated. With the exception of the joint pain of avascular necrosis, chronic pain syndromes in SCD have not been studied. In other chronic pain syndromes, central sensitization is thought to play a role. ${ }^{365}$ Hsieh and his colleagues $^{3}$ described four people on chronic daily opioid medications for sickle cell pain who were weaned off these medications after successful stem cell transplants. This approach suggests the possibility that a reversible process may be responsible for the chronic pain that so frequently occurs in SCD.

Pulmonary complications are common in SCD. One of the most serious problems is ACS, which often follows an acute vaso-occlusive crisis (VOC) and can complicate many surgeries. The manifestations of ACS include fever, chest pain, hypoxemia, cough and/or dyspnea, and a new infiltrate evident on chest $\mathrm{x}$ ray involving at least one lung segment. ${ }^{251}$ Potential etiologies of ACS include infection, bone marrow fat embolization, and in situ sickling with pulmonary infarction. ACS causes significant morbidity and is associated with higher risk of death. (For additional discussion of ACS, refer to page 46 in the "Managing Acute Complications of Sickle Cell Disease" chapter) 
Multiple genetic and environmental factors influence the degree of hemolysis and the occurrence of vasoocclusion in SCD. One of the best examples is the profoundly favorable effect that high fetal hemoglobin $(\mathrm{HbF})$ levels have on preventing intra-erythrocytic hemoglobin S polymerization and vaso-occlusion. The beneficial effects of genetically determined, persistent elevations of HbF levels in people who have SCD throughout their lifespan were documented through carefully conducted cohort studies in the 1970s and 1980s. ${ }^{142,366}$ These observations supported the concept that therapeutic interventions to increase HbF levels could improve clinical outcomes, especially pain and ACS, in people who have SCD. For example, 5-azacytidine was found to be capable of inducing $\mathrm{HbF}$ production in cell cultures, an effect confirmed in an animal model ${ }^{367}$ and in a few people who had thalassemia or SCD. Other drugs capable of increasing HbF levels were sought to permit oral administration and more acceptable toxicity profiles.

Hydroxyurea, a ribonucleotide reductase inhibitor, was identified as a promising drug candidate to increase $\mathrm{HbF}$ levels in people with SCD. Prior to its use in SCD, this medication has been in use for several decades to treat people with myeloproliferative disorders. Hydroxyurea is known to have rapid absorption and near-complete bioavailability and to be therapeutic with once-daily oral dosing. The initial clinical trial of hydroxyurea for the treatment of SCA involved two people. The study showed that short-term hydroxyurea therapy increased the number of HbF-containing reticulocytes and was not associated with short-term toxicities. ${ }^{368}$ This favorable result led to two carefully planned, extended studies testing the effects of hydroxyurea treatment in larger cohorts of people with SCA. Both of these clinical trials demonstrated that hydroxyurea was well tolerated and increased $\mathrm{HbF}$ levels in the majority of people. ${ }^{369,370}$ The results provided the necessary information to plan a major prospective phase III clinical trial to test the effects of hydroxyurea on clinical outcomes. ${ }^{371}$ Subsequently, numerous observational studies and three randomized trials in people with SCA were conducted. Limited data from observational studies are available on hydroxyurea therapy in people with genotypes other than $\mathrm{HbSS}$ or $\mathrm{HbS} \beta^{0}$-thalassemia.

Although $\mathrm{HbF}$ induction is the most powerful effect of hydroxyurea and provides the biggest direct benefit for people who have SCD, additional mechanisms of action and benefits exist. For example, hydroxyurea lowers the number of circulating leukocytes and reticulocytes and alters the expression of adhesion molecules, all of which contribute to vaso-occlusion. ${ }^{372}$ Hydroxyurea also raises RBC volume (higher mean corpuscular volume (MCV)) and improves cellular deformability and rheology, which increases blood flow and reduces vaso-occlusion. In addition, nitric oxide released directly from hydroxyurea metabolism may contribute to local vasodilation. ${ }^{373}$

\section{Methodology}

Complete information about the methodology for these guidelines can be found in the "Introduction and Methodology" chapter (pages 1-9). The following information, specific to this chapter, supplements the standard methodology that was conducted for all clinical chapters of these guidelines.

For this chapter, all human studies in English published from 2007 to May 2010 that addressed the PICOS question were identified. Studies published prior to 2007 were obtained from the 2008 National Institutes of Health Consensus Conference on Hydroxyurea document, "Hydroxyurea for the Treatment of Sickle Cell Disease," which included a systematic review. A total of 414 studies were included. ${ }^{i}$ In some cases in this chapter, a literature search was not conducted, so the panel relied on their cumulative expertise and knowledge to make recommendations; these recommendations are labeled "Consensus-Panel Expertise."

\footnotetext{
${ }^{i}$ An updated search was performed to span the time from June 1, 2010 through July 11, 2014. Two additional RCTs were identified, for a total of 414 studies, and a supplemental table reflecting these additions was added to the evidence table document.
} 
Detailed information on the evaluated studies as well as the observational and case studies/series referenced can be found in the Hydroxyurea for Sickle Cell Disease evidence table available at

http://www.nhlbi.nih.gov/guidelines/scd/index.htm.

\section{Summary of the Evidence}

A comprehensive systematic review was conducted evaluating the efficacy, effectiveness, harms, and barriers associated with using hydroxyurea in SCD. Three randomized trials and 54 observational studies describing the use of hydroxyurea in adults $(n=21)$ and children $(n=33)$ were identified. Exhibit 11 describes participant characteristics for the randomized trials, and exhibit 12 includes the evidence profile of efficacy/effectiveness for hydroxyurea in patients with SCA.

\section{Evidence of Efficacy/Effectiveness}

\section{Summary of Evidence in Adults With SCA}

The Multicenter Study of Hydroxyurea in Patients With Sickle Cell Anemia (MSH) was a randomized, doubleblind, placebo-controlled trial involving 299 adults with SCA who had experienced three or more VOCs in the previous year. The clinical end point of three or more documented VOCs was chosen because of earlier data documenting that people who experience pain at that frequency had markedly lower survival rates. ${ }^{142}$ The MSH trial demonstrated that, compared to placebo, hydroxyurea therapy reduced the frequency of painful episodes and ACS events, as well as the need for RBC transfusions and hospitalizations. ${ }^{373}$ In 1998, based on the results of this trial, the U.S. Food and Drug Administration approved hydroxyurea for the treatment of clinically severe SCA in adults.

\section{Summary of MSH Findings}

- Lower annual rates of pain crises (median 2.5 crises per year vs. 4.5 crises per year)

- Longer time to a first crisis on study (3.0 months vs. 1.5 months) and longer time to a second crisis (8.8 months vs. 4.6 months)

- Lower incidence of ACS (25 patients vs. 51 patients)

- Reduced need for blood transfusions (48 patients vs. 73 patients)

- Increased total hemoglobin $(0.6 \mathrm{~g} / \mathrm{dL})$ and $\mathrm{HbF}$ (from 5.0 to 8.6 percent in the intervention group), compared with a drop in the placebo group (from 5.2 to 4.7 percent)

- Lower costs for hospitalization for pain $(\$ 12,160$ in the hydroxyurea group versus $\$ 17,290$ in the placebo group)

- Differences in the effect on mortality and stroke outcomes were not statistically significant.

Over 2 years of treatment, the benefit of hydroxyurea on quality of life was limited to people who maintained increased $\mathrm{HbF}$ levels. These restricted benefits occurred in social function, pain recall, and general health perception. Annualized total costs were similar between the intervention group and the placebo group. The trial had low risk of bias but was stopped early for benefit, which may exaggerate the observed benefit. Supporting evidence from 21 observational studies involving 3,378 adults, with followup periods of 24-96 months, was consistent in showing a reduction in pain crises (60-90 percent), hospitalizations (90-100 percent), and an increase in $\mathrm{HbF}$ (4-20 percent).

A 9-year followup analysis of MSH participants indicated a reduction in mortality for the group of people who took hydroxyurea compared to those who did not take the medication. ${ }^{374}$ When the cohort was followed for up to 9 years, people taking hydroxyurea had 40 percent reduced mortality (analysis according to cumulative 
hydroxyurea exposure, not the original randomization). Survival was related to HbF levels and frequency of vaso-occlusive crises. More recently, extension of the followup analysis to 17.5 years for nonrandomized people indicated continued safety and benefit of hydroxyurea, including reduced mortality. ${ }^{375}$ Results were published from another prospective clinical study of hydroxyurea therapy with 17-year followup analysis. ${ }^{376}$ This prospective, nonrandomized study was conducted in Greece and enrolled people older than 16 years who had $\mathrm{HbSS}$ or $\mathrm{HbS}^{0}$-thalassemia, and $\mathrm{HbS}^{+}$-thalassemia. Similar to the results of the MSH trial, the results from this study showed that hydroxyurea therapy reduced the frequency of painful episodes and ACS events and the need for RBC transfusions and hospitalizations. Hydroxyurea therapy also significantly improved survival when compared to conventional therapy.

With randomized trials, both stopping the trial early and imprecision (single trial with $<300$ events) can affect the quality of the evidence. However, the overall quality of the evidence is considered high because the supporting observational evidence and the large treatment effect that follows hydroxyurea administration strengthen inference.

\section{Summary of Evidence in Children With SCA}

For infants, children, and adolescents who have SCA, hydroxyurea treatment results have closely and consistently mirrored those of adults. The first large, prospective, multicenter phase I/II trial (HUG KIDS) of school-aged children who were treated with hydroxyurea escalated to the maximum tolerated dose demonstrated laboratory efficacy, few short-term toxicities, and lack of toxicity for childhood growth and development. ${ }^{377}$ Soon after, a prospective phase I/II trial of infants with SCA who were treated with a liquid hydroxyurea formulation at a fixed dose of $20 \mathrm{mg} / \mathrm{kg} /$ day generated favorable short-term safety data and evidence suggesting prevention of sickle cell-related organ damage. ${ }^{378}$ Subsequently, several groups in the United States and Europe published open-label data regarding the laboratory and clinical efficacy of hydroxyurea for young people with SCA, with evidence of sustained laboratory and clinical responses but without apparent long-term toxicities. Taken together, these trials provide almost 15 years of pediatric data on both the safety and efficacy of hydroxyurea for young people (reviewed in Ware 2010). ${ }^{372}$ Most recently, the phase III double-blinded, placebo-controlled infant hydroxyurea study "Pediatric Hydroxyurea phase III Clinical Trial"379 had equivocal results for preservation of organ function, but confirmed the improvements in laboratory parameters such as total hemoglobin level and HbF level, and decreased numbers of sickle-related acute clinical events such as pain and ACS. ${ }^{380}$ Long-term observational studies suggest sustained beneficial effects of hydroxyurea for young people without excessive myelotoxicity, deleterious effects on growth and development, altered fertility, accumulation of mutations, or increased carcinogenicity. ${ }^{381-384}$

\section{Exhibit 11. Participant Enrollment Criteria for Placebo-Controlled Randomized Controlled Trials of Hydroxyurea Therapy in Sickle Cell Disease}

\begin{tabular}{|c|c|c|}
\hline Publication & Age Range & Clinical Characteristics \\
\hline Charache et al. $1995(\mathrm{MSH})^{373}$ & $>18 \mathrm{yr}$ & $\geq 3$ crises in $12 \mathrm{mo}$ \\
\hline Ferster et al. 1996385 & $2 \mathrm{yr}-22 \mathrm{yr}$ & $>3$ crises in $12 \mathrm{mo}$ \\
\hline Wang et al. 2011 (BABY HUG) 380 & $9 \mathrm{mo}-18 \mathrm{mo}$ & No restriction based on clinical severity \\
\hline
\end{tabular}




\section{Exhibit 12. Evidence Profile-Evidence of Efficacy/Effectiveness for Children and Adults With Sickle Cell Anemia (Hydroxyurea Versus Usual Care)}

\begin{tabular}{|c|c|c|}
\hline Outcome & Quality of the Evidence & Treatment Effect \\
\hline Pain crises & High & Statistically significant benefit \\
\hline Acute chest syndrome & Moderate & Statistically significant benefit \\
\hline $\begin{array}{l}\text { Hemoglobin level, fetal hemoglobin level, } \\
\text { need for blood transfusions }\end{array}$ & Moderate & Statistically significant benefit \\
\hline Mortality & Low & Imprecise estimate \\
\hline Stroke & Low & Imprecise estimate \\
\hline
\end{tabular}

\section{Summary of Evidence in People With Genotypes Other Than HbSS or HbS $\beta^{0}$-Thalassemia}

There have been no phase III trials of hydroxyurea therapy in people with SCD having genotypes other than $\mathrm{HbSS}$ or $\mathrm{HbS}^{0}$-thalassemia. The prospective, nonrandomized study from a major clinical center in Greece referred to earlier enrolled 165 people with $\mathrm{HbS}^{+}$-thalassemia. ${ }^{376}$ Only 44 people were receiving hydroxyurea. Data analysis was based on all subjects enrolled in the study, with the majority receiving hydroxyurea therapy represented by people with SCA. The overall 10-year survival for the subset of people with $\mathrm{HbS}^{+}$-thalassemia receiving hydroxyurea was not significantly different from those receiving conventional therapy. A phase II study of children and adults with HbSC evaluated the effects of hydroxyurea and magnesium pidolate on laboratory parameters. ${ }^{386}$ The study was closed due to slow enrollment, and only 36 people were evaluable for the primary outcome of proportion of hyperdense cells after 8 weeks; no difference was seen for people receiving hydroxyurea. In addition, people receiving hydroxyurea had favorable hematological effects with increased $\mathrm{HbF}$ and $\mathrm{RBC} \mathrm{MCV}$, which were not observed for people receiving only magnesium pidolate.

\section{Evidence of Side Effects}

The evidence for hydroxyurea toxicity in people with SCD is derived from three RCTs that enrolled 517 people and from 47 observational studies that enrolled more than 3,000 people. In people who do not have SCD, toxicity evidence is derived from 21 RCTs that enrolled more than 4,800 individuals and 35 observational studies that enrolled more than 7,500 individuals (see exhibit 13). (For more information, see the evidence table at http://www.nhlbi.nih.gov/guidelines/scd/index.htm).

\section{Exhibit 13. Evidence Profile-Evidence of Side Effects in Sickle Cell Anemia}

\begin{tabular}{|c|c|c|}
\hline Potential Toxicity & Quality of the Evidence & Treatment Effect \\
\hline Bone marrow suppression & High & Reversible cytopenias associated with hydroxyurea \\
\hline Leukemia & $\begin{array}{l}\text { No supporting evidence in SCD } \\
\text { populations/Very low }\end{array}$ & $\begin{array}{l}\text { The available evidence does not support the association of } \\
\text { hydroxyurea treatment with the development of leukemia in } \\
\text { adults or children }\end{array}$ \\
\hline Leg ulcers & $\begin{array}{l}\text { Adults: Moderate } \\
\text { Children: Low }\end{array}$ & $\begin{array}{l}\text { The available evidence does not support the association of } \\
\text { hydroxyurea treatment with leg ulcers }\end{array}$ \\
\hline Other side effects & Very low & $\begin{array}{l}\text { Numerous other side effects were reported in the literature } \\
\text { with low frequency and none with certain causality }\end{array}$ \\
\hline Reproductive effects & Very low & $\begin{array}{l}\text { Minimal human data exist on potential harmful reproductive } \\
\text { effects of hydroxyurea in males and females }\end{array}$ \\
\hline
\end{tabular}




\section{Evidence Supporting Use of a Treatment Protocol}

Although the literature does not compare different implementation protocols for hydroxyurea, the expert panel was concerned that not using a protocol could lead to inadequate dosing or poor monitoring. Hence, to maximize the benefits and safety of hydroxyurea treatment, the expert panel strongly recommends adopting a standardized protocol based on the available evidence. The expert panel developed a suggested protocol based on (1) protocols used in published clinical trials and observational studies, (2) indirect evidence derived from basic science and pharmacokinetics of hydroxyurea, and (3) a consensus process. Although the protocol contains several technical remarks and recommendations needed to implement hydroxyurea therapy safely and effectively, it should be considered as guidance and modified to fit an individual patient's clinical situation (see "Consensus Treatment Protocol and Technical Remarks for the Implementation of Hydroxyurea Therapy").

\section{Additional Considerations}

Guideline developers consider what is known in the literature about people's values and preferences and make assumptions about values demonstrated by people encountered in clinical practice. In the area of SCD, the evidence supporting the nature and distribution of people's values is not strong. However, the expert panel has considered patients' values in their decisionmaking process. In one study of pediatric patients and their caregivers, parents and children indicated a preference for hydroxyurea over other therapies such as routine RBC transfusions or stem cell transplantation. The benefit/harm balance seems to be the driving determinant of treatment choice in this study. ${ }^{387}$ In developing these recommendations, the expert panel placed high value on preventing SCD morbidity (specifically, VOCs and ACS) and low value on cost, burden, and the potentially unknown long-term adverse effects of hydroxyurea therapy.

Although the hydroxyurea clinical trials cited in this chapter used very restrictive definitions for chronic, acute, and recurrent pain, the panel has chosen to broaden the definitions by using information from people in observational studies and clinical trials. For example, for this document, the expert panel defined recurrent SCD-associated pain to include daily pain requiring the use of opioid medication. In addition, the expert panel also includes those people who have episodes of pain, which, in the view of the patient and provider, significantly affect activities of daily living and quality of life.

It is the nature of most efficacy clinical trials to restrict enrollment to people with substantial clinical severity. Unfortunately, this limits data on the majority of people who do not fit easily into the restrictive clinical trial definitions - that is, the people who are seen in everyday practice. Notably, the pediatric phase III trial (BABY $\mathrm{HUG})^{379}$ did not have specific inclusion criteria based on clinical severity; even infants with no previous clinical VOCs were eligible for enrollment. This was intentional and designed to allow the findings to be generalized to all infants and toddlers with SCA. ${ }^{380}$

The panel deliberated extensively on using data from clinical trials alone, which in most cases would limit the use of hydroxyurea to people who have had three or more pain crises in the last year. However, the panel felt that this would prevent the use of hydroxyurea in some adults who have chronic, acute, and recurrent pain, and for whom observational studies have generally shown a benefit from the medication. Therefore, in an effort to include the broad range of pain syndromes that affect the ability of people with SCD to participate in their desired daily activities, the panel's definitions of chronic, acute, and recurrent pain and their recommendations for the use of hydroxyurea have been expanded beyond the eligibility criteria used in the clinical trials. Thus, the expert panel believes that the use of hydroxyurea is indicated in a broader range of individuals than those described in the inclusion criteria for MSH and hopes to encourage use of the medication in people who have acute and/or chronic pain that regularly interferes with their quality of life. 
In addition, when issuing recommendations for adults, the expert panel occasionally used data from the pediatric SCD literature and data from populations without SCD who were treated with hydroxyurea. In particular, this occurred in the areas of evidence of harm and treatment initiation and monitoring. The panel acknowledges that this indirect evidence is of lower quality and associated with weaker inferences.

\section{Hydroxyurea Treatment Recommendations}

\section{Recommendations}

1. Educate all patients with SCA and their family members about hydroxyurea therapy. (See consensus treatment protocol on page 145).

(Consensus-Panel Expertise)

2. In adults with SCA who have three or more sickle cell-associated moderate to severe pain crises in a 12-month period, treat with hydroxyurea.

(Strong Recommendation, High-Quality Evidence)

3. In adults with SCA who have sickle cell-associated pain that interferes with daily activities and quality of life, treat with hydroxyurea.

(Strong Recommendation, Moderate-Quality Evidence)

4. In adults with SCA who have a history of severe and/or recurrent ACS, treat with hydroxyurea. ${ }^{*}$ (Strong Recommendation, Moderate-Quality Evidence)

5. In adults with SCA who have severe symptomatic chronic anemia that interferes with daily activities or quality of life, treat with hydroxyurea.

(Strong Recommendation, Moderate-Quality Evidence)

6. In infants 9 months of age and older, children, and adolescents with SCA, offer treatment with hydroxyurea regardless of clinical severity to reduce SCD-related complications (e.g., pain, dactylitis, ACS, anemia).

(Strong Recommendation, High-Quality Evidence for ages 9-42 months; Moderate Recommendation, Moderate-Quality Evidence for children $>42$ months and adolescents). Note: The panel intentionally used the term "offer" realizing that patients' values and preferences may differ particularly considering treatment burden (e.g., laboratory monitoring, office visits), availability of drug in a liquid form, and cost. Therefore, the panel strongly encourages shared decisionmaking and discussion of hydroxyurea therapy with all patients.

7. In adults and children with SCD who have chronic kidney disease and are taking erythropoietin, hydroxyurea therapy can be added to improve anemia.

(Weak Recommendation, Low-Quality Evidence)

8. In females who are pregnant or breastfeeding, discontinue hydroxyurea therapy.

(Moderate Recommendation, Very Low-Quality Evidence)

9. To ensure proper use of hydroxyurea and maximize benefits and safety, use an established prescribing and monitoring protocol.

(Strong Recommendation, High-Quality Evidence)

10. In people with $\mathrm{HbS}^{+}$-thalassemia or $\mathrm{HbSC}$ who have recurrent sickle cell-associated pain that interferes with daily activities or quality of life, consult a sickle cell expert for consideration of hydroxyurea therapy.

(Moderate Recommendation, Low-Quality Evidence)

11. In people not demonstrating a clinical response to appropriate doses and duration of hydroxyurea therapy, consult a sickle cell expert.

(Moderate Recommendation, Very Low-Quality Evidence)

* For more information, see the ACS section of the "Managing Acute Complications of Sickle Cell Disease" chapter. 


\section{Consensus Treatment Protocol and Technical Remarks for the Implementation of Hydroxyurea Therapy}

The following laboratory tests are recommended before starting hydroxyurea:

- Complete blood count (CBC) with white blood cell (WBC) differential, reticulocyte count, platelet count, and RBC MCV

- Quantitative measurement of $\mathrm{HbF}$ if available (e.g., hemoglobin electrophoresis, high-performance liquid chromatography (HPLC))

- Comprehensive metabolic profile, including renal and liver function tests

- Pregnancy test for women

\section{Initiating and Monitoring Therapy}

- Baseline elevation of $\mathrm{HbF}$ should not affect the decision to initiate hydroxyurea therapy.

- Both males and females of reproductive age should be counseled regarding the need for contraception while taking hydroxyurea.

- Starting dosage for adults (500 mg capsules): $15 \mathrm{mg} / \mathrm{kg} / \mathrm{day}$ (round up to the nearest $500 \mathrm{mg}$ ); $5-10 \mathrm{mg} / \mathrm{kg} / \mathrm{day}$ if patient has chronic kidney disease

- Starting dosage for infants and children: $20 \mathrm{mg} / \mathrm{kg} /$ day

- Monitor CBC with WBC differential and reticulocyte count at least every 4 weeks when adjusting dosage.

- Aim for a target absolute neutrophil count $\geq 2,000 / u L$; however, younger patients with lower baseline counts may safely tolerate absolute neutrophil counts down to 1,250/uL.

- Maintain platelet count $\geq 80,000 / \mathrm{uL}$

- If neutropenia or thrombocytopenia occurs:

- Hold hydroxyurea dosing

- Monitor CBC with WBC differential weekly

- When blood counts have recovered, reinstitute hydroxyurea at a dose $5 \mathrm{mg} / \mathrm{kg} / \mathrm{day}$ lower than the dose given before onset of cytopenias

- If dose escalation is warranted based on clinical and laboratory findings, proceed as follows:

- Increase by $5 \mathrm{mg} / \mathrm{kg} /$ day increments every 8 weeks

- Give until mild myelosuppression (absolute neutrophil count 2,000/uL to 4,000/uL) is achieved, up to a maximum of $35 \mathrm{mg} / \mathrm{kg} / \mathrm{day}$.

- Once a stable dose is established, laboratory safety monitoring should include:

- $\quad$ CBC with WBC differential, reticulocyte count, and platelet count every 2-3 months

- People should be reminded that the effectiveness of hydroxyurea depends on their adherence to daily dosing. They should be counseled not to double up doses if a dose is missed.

- A clinical response to treatment with hydroxyurea may take 3-6 months. Therefore, a 6- month trial on the maximum tolerated dose is required prior to considering discontinuation due to treatment failure, whether due to lack of adherence or failure to respond to therapy.

- Monitor RBC MCV and HbF levels for evidence of consistent or progressive laboratory response.

- A lack of increase in MCV and/or HbF is not an indication to discontinue therapy.

- For the patient who has a clinical response, long-term hydroxyurea therapy is indicated.

- Hydroxyurea therapy should be continued during hospitalizations or illness. 


\section{Chapter 6: Blood Transfusion in the Management of Sickle Cell Disease}

\section{Introduction}

Donor erythrocyte (red blood cell, RBC) transfusion was the first therapy used in SCD that targets the pathophysiology of SCD, even though transfusion was used before the basic pathophysiology of SCD was understood. ${ }^{388}$ Donor erythrocytes contain normal hemoglobin $(\mathrm{HbA})$, and transfusion of these donor RBCs into people with SCD reduces the percentage of circulating erythrocytes with abnormal hemoglobin (HbS). Although donor erythrocyte transfusion can ameliorate and even prevent SCD manifestations in select circumstances, transfusion of donor erythrocytes is not universally beneficial in SCD. Many of the recognized hazards of transfusion, such as the risk of alloimmunization, are amplified in $\mathrm{SCD}^{389}$; therefore, decisions to utilize transfusion therapy in SCD must be based on risk-benefit assessments. The goal of this chapter is to present evidence-based recommendations that summarize the indications, risks, and benefits of erythrocyte transfusion therapy in SCD.

\section{Background}

Sickled erythrocytes possess many unfavorable physiologic properties and induce vascular changes that promote vaso-occlusion. Sickled erythrocytes increase blood viscosity through intrinsic properties of the sickled cells as well as abnormal interactions of these cells with leukocytes, platelets, vascular endothelium, and clotting factors. Transfusion of donor erythrocytes is used in some situations to mitigate these effects with favorable results. Over the last three decades, the number of evidence-based indications for erythrocyte transfusion in SCD has been increasing. Ongoing studies of transfusion in SCD may provide evidence for additional indications for transfusion. As erythrocyte transfusion becomes more commonplace in the care of people with SCD, it is important to understand the evidence that supports its use in specific clinical situations.

To minimize adverse effects of transfusion, the selection and infusion of erythrocyte units should follow standard blood banking and transfusion practices. Many institutions provide all people with SCD with erythrocyte units that are sickle negative and leukocyte reduced; to prevent alloimmunization, many institutions also routinely provide units matched for minor Rh and Kell antigens. ${ }^{390-392}$ The clinical benefits of transfusing sickle-negative RBCs in SCD (as compared to sickle trait RBCs) have not been specifically studied. Transfusing sickle-negative erythrocytes assists with accurate tracking of percent sickled hemoglobin when specific HbS targets are used and avoids the possibility of the transfused erythrocytes becoming sickled, which has been described in extreme circumstances. ${ }^{393,394}$ A specific request of the blood bank may be required to obtain sickle-negative erythrocytes.

Sickle cell anemia (SCA) refers to the clinically similar disorders $\mathrm{HbSS}$ or $\mathrm{HbS} \beta^{0}$-thalassemia. Sickle cell disease (SCD) refers to all disease genotypes, including SCA and compound heterozygous disorders, such as HbSC, HbSD, and HbS $\beta+$-thalassemia. The carrier state for hemoglobin $\mathrm{S}$ (HbAS or sickle cell trait) is not a form of SCD. 
Approximately 70 percent of RBC units currently collected in the United States are leukoreduced before storage, ${ }^{395}$ and, by extension most RBC units given to people with SCD are leukoreduced. The benefits of transfusing leukoreduced erythrocytes in SCD have not been studied specifically, although previously documented benefits of leukoreduction in other populations include lower incidences of febrile nonhemolytic transfusion reactions, ${ }^{396}$ cytomegalovirus (CMV) transmission, ${ }^{397}$ and Human Leukocyte Antigen (HLA) alloimmunization. ${ }^{398}$ The benefits of using leukocyte-reduced erythrocytes are expected to be applicable to individuals with SCD who require transfusion.

Donor erythrocytes may be administered as a simple transfusion or as an exchange transfusion. Simple transfusion is the infusion of donor erythrocytes without removal of recipient blood, whereas exchange transfusion involves removal of recipient blood before and/or during donor erythrocyte infusion. Three benefits of exchange transfusion, related primarily to the removal of recipient sickle erythrocytes, include (1) increasing the percent of normal (donor) hemoglobin ( $\mathrm{HbA}$ )-containing erythrocytes remaining after transfusion;

(2) permitting transfusion of increased volumes of donor blood without increasing the hematocrit to levels that excessively increase blood viscosity; and (3) reducing the net transfused volume, which reduces iron overload. However, potential risks of exchange transfusion include (1) increased donor unit exposure and subsequent alloimmunization; (2) higher costs; (3) the need for specialized equipment; and (4) the frequent need for permanent venous access. Exchange transfusion can be accomplished by manual or automated (erythrocytapheresis) methods. The decision regarding the type of transfusion technique to employ is multifactorial and is guided by patient acuity, institutional expertise, and compatible blood supply.

Erythrocyte transfusion in SCD can be further classified as episodic or chronic. Episodic transfusion is used either acutely in response to a complication of SCD or prophylactically in preparation for anesthesia or surgery. Chronic transfusion is used when sustained, low levels of $\mathrm{HbS}$ are needed for primary or secondary prophylaxis for SCD complications, most commonly stroke in children. Chronic transfusion programs may use different blood matching and monitoring strategies, although the themes of avoiding transfusion reactions, minimizing alloimmunization, maintaining low $\mathrm{HbS}$ levels, and tracking and treating transfusional iron overload are common among all programs. ${ }^{399}$

Transfusions can be lifesaving and protect organs from ongoing damage from sickled erythrocytes in the appropriate setting but also can cause serious and occasionally life-threatening complications. Judicious application of erythrocyte transfusion therapy in SCD requires an understanding of the evidence for erythrocyte transfusion in specific clinical situations and an understanding of the additional risks of transfusion in people with SCD.

There are many potential indications for transfusion in the patient with SCD. This chapter discusses only the most common indications, including prophylactic perioperative transfusion; transfusion in the setting of acute occurrences such as stroke, multisystem organ failure, and acute chest syndrome (ACS); and transfusion in the setting of chronic occurrences such as primary and secondary prevention of stroke in children. Common transfusion side effects, including alloimmunization, autoimmunization, iron overload, hyperviscosity, and hemolysis, are also discussed.

\section{Methodology}

Complete information about the methodology for these guidelines can be found in the "Introduction and Methodology" chapter (pages 1-9). The following information, specific to this chapter, supplements the standard methodology that was conducted for all clinical chapters of these guidelines. 
A comprehensive study of several databases was conducted, and all human studies in English published from 1970 to July 2010 that addressed each PICOS question were identified. A total of 300 studies were included. ${ }^{j}$ In some cases in this chapter, a literature search was not conducted or the search yielded no evidence (e.g., management of hyperviscosity), so the expert panel relied on their cumulative expertise and knowledge to make recommendations; these recommendations are labeled "Consensus-Panel Expertise." The key questions for this chapter can be found immediately before the Summary of the Evidence sections for the individual topics.

Detailed information on the evaluated studies as well as the observational and case studies/series referenced can be found in the Transfusion in Sickle Cell Disease evidence table available at http://www.nhlbi.nih.gov/guidelines/scd/index.htm.

\section{Indications for Transfusion}

\section{Prophylactic Perioperative Transfusion}

\section{Background}

Transfusions are commonly used in the perioperative period to prevent the development of vaso-occlusive crises (VOCs), stroke, or ACS after surgery. Surgical procedures are associated with significant morbidity for individuals with SCD. In the Cooperative Study of Sickle Cell Disease (CSSCD), sickle-related complications (e.g., VOC, ACS, and stroke) occurred in 0-18.6 percent of patients with SCA (depending on the surgical procedure) and non-sickle cell-related complications (defined as fever, infection, bleeding, thrombosis, embolism, and death) occurred in 5.7 to 26.2 percent of patients. There were 12 deaths in 1,079 surgical cases. $^{400}$

The Transfusion Alternatives Preoperatively in Sickle Cell Disease (TAPS) study was published in 2013 and thus was not included in the evidence review. However, the expert panel did want to acknowledge this important study. TAPS was a multicenter trial in Europe and Canada ${ }^{401}$ that randomized individuals with SCA to either no preoperative transfusion or preoperative transfusion. The study enrolled patients undergoing lowrisk (e.g., adenoidectomy, inguinal hernia repair) and medium-risk (e.g., cholecystectomy, joint replacement) procedures. The goal of transfusion was to raise the hemoglobin to $10.0 \mathrm{~g} / \mathrm{dL}$. In patients with preoperative hemoglobin levels of $9.0 \mathrm{~g} / \mathrm{dL}$ or higher, a partial exchange transfusion was done. The study was closed early due to significantly more complications in the medium-risk, no preoperative transfusion arm than in the medium-risk transfusion arm (10/33 vs. 1/34). The unadjusted odds ratio of clinically important complications was 3.8. As the study was closed early, the estimate observed may be overestimated. There were too few patients enrolled in the low-risk procedure arms to draw any conclusions.

\section{Key Question}

\section{KQ25. In patients with SCD undergoing surgical procedures, does a particular perioperative transfusion approach (simple or exchange transfusion to achieve a predetermined hemoglobin level or percentage of $\mathrm{HbS}$ ) reduce perioperative mortality and complications?}

\footnotetext{
${ }^{\mathrm{j}}$ An updated search was performed to span the time from June 1, 2010 through July 11, 2014. One additional RCT was identified, for a total of 300 studies, and a supplemental table reflecting this addition was added to the evidence table document.
} 


\section{Summary of the Evidence}

One RCT, four observational studies, and six case series evaluated perioperative transfusion outcomes in SCD. Overall, the quality of evidence was low due to severe imprecision (small number of events) and lack of controlled comparisons; therefore, the results are inconclusive.

A single randomized trial has been done examining the use of transfusion in the perioperative period. ${ }^{402}$ This study compared the use of simple transfusion with a hemoglobin $(\mathrm{Hb})$ goal of $10 \mathrm{~g} / \mathrm{dL}$ preoperatively to the use of exchange transfusion to bring the $\mathrm{HbS} \leq 30$ percent. No statistically significant reduction in the incidence of perioperative complications was seen between the two arms of the study, although complication rates in both arms were high (31 percent in the exchange group and 35 percent in the simple transfusion group), and 10 percent of patients in both arms developed ACS. ${ }^{402}$

The four observational studies and six case series reported on various outcomes of transfusion in the perioperative period. In 717 patients with SCA undergoing surgical procedures in the CSSCD, the combined incidence of all sickle cell-related complications postoperatively was significantly lower in those who had preoperative transfusion compared to those who did not have transfusion. ${ }^{400}$ Similar results were demonstrated in individuals with sickle hemoglobin $\mathrm{C}(\mathrm{HbSC})$ disease. $^{400}$

Al-Samak et al. ${ }^{403}$ described 46 patients who underwent simple transfusion, exchange transfusion, and no transfusion. The incidence of sickle cell crisis and ACS was similar in all three groups. Wali et al. ${ }^{404}$ studied 14 patients and reported similar perioperative outcomes of conservative versus aggressive transfusion $(\mathrm{Hb}>10 \mathrm{~g} / \mathrm{dL}$ and $\mathrm{HbS}<30$ percent). The remaining seven studies were uncontrolled case series and did not provide additional conclusions.

\section{Recommendations}

1. In adults and children with SCA, transfuse RBCs to bring the hemoglobin level to $10 \mathrm{~g} / \mathrm{dL}$ prior to undergoing a surgical procedure involving general anesthesia.

(Strong Recommendation, Moderate-Quality Evidence)

2. In patients with $\mathrm{HbSS}$ disease who require surgery and who already have a hemoglobin level higher than $8.5 \mathrm{~g} / \mathrm{dL}$ without transfusion, are on chronic hydroxyurea therapy, or who require high-risk surgery (e.g., neurosurgery, prolonged anesthesia, cardiac bypass), consult a sickle cell expert for guidance as to the appropriate transfusion method.

(Strong Recommendation, Low-Quality Evidence)

3. In adults and children with $\mathrm{HbSC}_{\mathrm{br}} \mathrm{HbSB}^{+}$-thalassemia, consult a sickle cell expert to determine if full or partial exchange transfusion is indicated before a surgical procedure involving general anesthesia.

(Moderate Recommendation, Low-Quality Evidence) 


\section{Recommendations for Acute and Chronic Transfusion Therapy}

Exhibits 14-19 summarize the expert panel's recommendations for transfusion therapy in acute and chronic complications. A more detailed discussion of the indications for transfusion and the evidence to support these recommendations for each disorder can be found in the chapters on health maintenance, acute complications, or chronic complications. Exhibits 14-19 are designated as either graded or consensus recommendations.

\section{Exhibit 14. Acute Complications-Graded Recommendations To Transfuse}

\begin{tabular}{|l|l|c|c|}
\multicolumn{1}{c}{ Indication } & \multicolumn{1}{c}{ How To Transfuse } & \multicolumn{2}{c|}{$\begin{array}{c}\text { Quality of Evidence } \\
\text { Recommendation }\end{array}$} \\
\hline $\begin{array}{l}\text { Symptomatic acute chest } \\
\text { syndrome (ACS) combined } \\
\text { with a decreased Hb of } \\
1 \mathrm{~g} / \mathrm{dL} \text { below baseline }\end{array}$ & Simple transfusion & Low & Weak \\
\hline $\begin{array}{l}\text { Symptomatic severe ACS } \\
\text { (as defined by an oxygen } \\
\text { saturation less than } 90 \% \\
\text { despite supplemental } \\
\text { oxygen) }\end{array}$ & Exchange transfusion & Low & Strong \\
\hline $\begin{array}{l}\text { Acute splenic sequestration } \\
\text { plus severe anemia }\end{array}$ & Simple transfusion & Low & \\
\hline Stroke & $\begin{array}{l}\text { Simple or exchange } \\
\text { transfusion }\end{array}$ & Low & Strong \\
\hline
\end{tabular}

\section{Exhibit 15. Acute Complications-Consensus Recommendations To Transfuse}

\section{Indication}

\section{How To Transfuse}

\begin{tabular}{|l|l|}
\hline Hepatic sequestration & Exchange or simple transfusion \\
\hline Intrahepatic cholestasis & Exchange or simple transfusion \\
\hline Multisystem organ failure (MSOF) & Exchange or simple transfusion \\
\hline Aplastic crisis & Simple transfusion \\
\hline $\begin{array}{l}\text { Symptomatic anemia (see page 43 in the "Managing Acute } \\
\text { Complications of Sickle Cell Disease" chapter) }\end{array}$ & Simple transfusion \\
\hline
\end{tabular}

\section{Exhibit 16. Acute Complications-Graded Recommendations When Transfusion Is Not Indicated}
Indication
Quality of Evidence
Strength of Recommendation

Uncomplicated painful crisis

Low

Moderate

Priapism

Low

Moderate 


\section{Exhibit 17. Acute Complications-Consensus Recommendations When Transfusion Is Not Indicated}

Indication

- Asymptomatic anemia

- Acute kidney injury, unless multisystem organ failure (MSOF)

\section{Exhibit 18. Chronic Complications-Graded Recommendations for When To Initiate a Chronic Transfusion Program}

\begin{tabular}{|c|c|c|c|}
\hline Indication & How To Transfuse & Quality of Evidence & $\begin{array}{c}\text { Strength of } \\
\text { Recommendation }\end{array}$ \\
\hline $\begin{array}{l}\text { Child with transcranial Doppler (TCD) reading* } \\
>200 \mathrm{~cm} / \mathrm{sec}\end{array}$ & $\begin{array}{l}\text { Exchange or simple } \\
\text { transfusion }\end{array}$ & High & Strong \\
\hline $\begin{array}{l}\text { Adults and children with previous clinically overt } \\
\text { stroke }\end{array}$ & $\begin{array}{l}\text { Exchange or simple } \\
\text { transfusion }\end{array}$ & Low & Moderate \\
\hline
\end{tabular}

* TCD reading is the time averaged mean maximal cerebral blood flow velocity. See section about Screening for Risk of Stroke Using Neuroimaging in the "Health Maintenance for People With Sickle Cell Disease" chapter.

\section{Exhibit 19. Chronic Complications-Graded Recommendations for When Transfusion is Not Indicated}

Indication

Recurrent splenic sequestration
Quality of Evidence

Low
Strength of Recommendation

Weak

\section{Appropriate Management/Monitoring}

The administration of RBC transfusions is common in both children and adults with SCD. In this area, the expert panel reviewed literature to answer questions about phenotype matching, the goals of transfusion therapy, and appropriate monitoring in chronically transfused individuals. Studies have tried to answer whether giving phenotypically matched red cells decreases the risk of alloimmunization in people with SCD. In addition, questions have arisen about the appropriate transfusion goals for patients undergoing transfusion both acutely and chronically. The expert panel was able to make recommendations for goals of chronic transfusion therapy in children, but evidence was insufficient to propose a goal $\mathrm{HbS}$ concentration for chronically transfused adults, as it may vary by indication.

This section concludes with a consensus-based protocol on appropriate monitoring of patients who receive chronic transfusions. The protocol contains several technical remarks and recommendations needed to implement chronic transfusion therapy safely and effectively. The protocol should be considered as guidance and modified to fit an individual patient's clinical situation. 


\section{Key Question}

KQ26. In patients with SCA who require RBC transfusion, what are the most effective transfusion protocols that reduce transfusion complications (including a transfusion goal, phenotype-matching monitoring approaches, procedures, or strategies)?

\section{Summary of the Evidence}

\section{Phenotype Matching}

Four RCTs, 63 longitudinal and cross-sectional studies, and 46 case reports were identified that demonstrated alloimmunization. In the four RCTs (with $>1,100$ patients), alloimmunization/autoimmunization development rates ranged between 3 percent and 29 percent. ${ }^{98,402,405,406}$ In the other 63 studies (involving $>6,000$ patients), alloimmunization rates ranged between 6 percent and 85 percent, and autoimmunization rates ranged between 4 percent and 10 percent. Overall, minimal evidence is available to support a particular method to reduce or prevent side effects from RBC transfusion. ${ }^{407}$

The systematic review did not identify comparative effectiveness studies that explored different cross-matching approaches. Two studies (one RCT and one observational study involving 159 patients) that implemented stricter matching criteria had more favorable results (alloimmunization rates $<7$ percent) ${ }^{408,409}$ The definition of a "strict cross match" varied among studies, and often included matching for ABO and a number of other RBC antigens, including DCcEe and Kell, and occasionally Kidd and Duffy. ${ }^{409}$ In the published studies, to prevent alloimmunization or to transfuse patients who were already alloimmunized, investigators most commonly opted to use strictly phenotype-matched RBC units.

\section{Transfusion Goals}

The systematic review did not identify evidence supporting the effectiveness of a specific HbS percentage cutoff for transfusion (i.e., there are no comparative studies in which different $\mathrm{HbS}$ targets were evaluated). In the transfusion protocols used in the included randomized trials of patients treated with chronic transfusion, two (both in children) used a cutoff of $\leq 30$ percent (STOP 1 and 2), ${ }^{96,98}$ while the remaining trial, which studied the use of chronic transfusion in pregnancy, used a cutoff of hemoglobin between $10 \mathrm{~g} / \mathrm{dL}$ and $11 \mathrm{~g} / \mathrm{dL}^{405}$ and a HbS cutoff of $\leq 35$ percent. The $\leq 30$ percent cutoff was used in roughly 75 percent of the observational studies (a total of 2,648 adults and 4,523 children). However, it is unclear how the use of these cutoffs correlates with outcomes. In the two multicenter stroke prevention trials, this cutoff was beneficial in reducing the risk of stroke (compared to no transfusion). These data may guide the practice of transfusion in SCD and suggest a particular transfusion goal; however, the evidence is indirect and of low quality.

No studies evaluated the effectiveness of different monitoring strategies. 


\section{Recommendations}

1. RBC units that are to be transfused to individuals with $S C D$ should include matching for $C, E$, and $K$ antigens. (Moderate Recommendation, Low-Quality Evidence)

2. In patients with SCA, who are not chronically transfused and who are therefore at risk for hyperviscosity due to high percentages of circulating HbS-containing erythrocytes, avoid transfusing to a target hemoglobin above $10 \mathrm{~g} / \mathrm{dL}$. (Moderate Recommendation, Low-Quality Evidence)

3. In chronically transfused children with SCA, the goal of transfusion should be to maintain a HbS level of below 30 percent immediately prior to the next transfusion.

(Moderate Recommendation, Moderate-Quality Evidence)

4. The expert panel recommends that clinicians prescribing chronic transfusion therapy follow an established monitoring protocol.

(Moderate Recommendation, Low-Quality Evidence)

Although the literature does not offer evidence comparing different implementation protocols for chronic transfusion therapy, the expert panel was concerned about inadequate monitoring if a protocol is not used. Hence, taking into account the evidence supporting the use of routine monitoring, the expert panel issued a recommendation for adopting a standardized protocol to maximize benefits and safety. A suggested protocol was developed by the expert panel based on (1) protocols used in the published clinical trials and observational studies, (2) indirect evidence derived from basic science, and (3) a consensus process. The protocol contains several technical remarks and recommendations needed to implement chronic transfusion therapy safely and effectively, but the protocol should be considered as guidance and modified to fit an individual patient's clinical situation. 
The following is a consensus protocol for the initiation and monitoring of patients on chronic transfusion therapy. It is understood that the recommended testing schedule may not be available to patients everywhere; therefore, this protocol should serve only as a helpful guide for transfusion management.

\section{At Initiation}

- Obtain patient treatment history to include locations where prior transfusions were received and any adverse effects.

- Notify the blood bank that the patient being initiated on chronic transfusion therapy has SCD. Ask the blood bank to contact hospitals where the patient reported receiving previous transfusion therapy to obtain transfusion information.

- Obtain a RBC phenotype, type and screen, quantitative measurement of percent $\mathrm{HbA}$ and percent $\mathrm{HbS}$, complete blood count ( $\mathrm{CBC})$, and reticulocyte count.

- Inform the patient if he or she is alloimmunized, so that this information can be communicated as part of the patient's self-reported medical history.

\section{Suggested Evaluation Before Each Transfusion}

- $\quad \mathrm{CBC}$ and reticulocyte count-This procedure is done to help guide the frequency and volume of transfusions. It is expected that, with effective chronic transfusion therapy, the patient's bone marrow will be suppressed and the reticulocyte count should decrease, but the value may rise by the time of the next transfusion.

- Quantitative measurement of percent $\mathrm{HbA}$ and percent $\mathrm{HbS}$-This procedure is done to confirm the success of chronic transfusion therapy with achieving the target percent of $\mathrm{HbS}$.

- Type and screen-This is done to assess whether the patient has developed any new RBC antibodies from the prior transfusion.

\section{Suggested Periodic Evaluations}

- Liver function tests annually or semiannually-These tests are done to follow liver function in individuals with iron overload.

- Serum ferritin (SF) quarterly-This test is done to follow iron stores in individuals with iron overload; it can be helpful in evaluating compliance with chelation.

- Screening for hepatitis C, hepatitis B, and HIV annually.

- Evaluation for iron overload every 1-2 years by validated liver iron quantification methods such as liver biopsy, MRI $\mathrm{R} 2$ or MRI T2* or R2 techniques.

\section{Complications of Transfusions}

\section{Overview}

Although RBC transfusions can help ameliorate many of the acute and chronic complications of SCD - and, at times, can be life-saving - their administration is associated with a wide variety of complications. Some transfusion-associated events are relatively mild, while others can be severe or even fatal. Health care providers should become familiar with the range of transfusion complications and learn their signs and symptoms as well as appropriate diagnostic testing, prevention strategies, and therapeutic interventions when warranted. 
This section discusses alloimmunization, autoimmunization, iron overload, hemolysis, and hyperviscosity - the most commonly occurring side effects of transfusion. After a description of the side effects and a summary of the evidence, this section concludes by identifying some areas in which additional research is needed.

\section{Alloimmunization and Autoimmunization}

\section{Background}

Human erythrocytes express a large number of surface proteins, glycoproteins, polysaccharides, and glycolipids that are potentially immunogenic. Following an erythrocyte transfusion, if the donor erythrocytes have a different antigenic profile from those of the recipient's own erythrocytes, an immunological response by the recipient against the "foreign" antigens can result in a process known as alloimmunization. Polysaccharide antigens generally elicit only immunoglobulin $\mathrm{M}(\operatorname{IgM})$ responses, but other erythrocyte antigens elicit an immune response that begins with production of polyclonal IgM alloantibodies within 3-7 days of antigenic stimulation and then evolves to polyclonal IgG alloantibodies over several weeks.

Immunoglobulin $\mathrm{G}(\mathrm{IgG})$ alloantibodies persist for many years, although their titer may wane to low or undetectable levels. Almost all IgM alloantibodies, and some IgG alloantibodies, can bind to the transfused erythrocytes and fix complement, a set of serum proteins that bind to the erythrocyte and cause direct hemolysis. The result of alloimmunization is usually destruction of transfused erythrocytes that express the antigen, but the pathophysiology of red blood cell destruction and immune-mediated clearance is complex and depends upon several features including the antibody isotype, titer, and ability to fix complement. Occasionally, the recipient's own erythrocytes become immunogenic and stimulate an immune response known as autoimmunization; most autoantibodies are IgG, and some fix complement. Autoantibody formation can occur at any time but occurs most frequently in patients who have already developed multiple alloantibodies. Alloimmunization usually limits the ability to find compatible blood for future transfusions and increases the risk for delayed hemolytic transfusion reactions, so efforts to avoid alloantibodies seem warranted.

\section{Key Question}

\section{KQ27. In patients with SCD requiring transfusion, what are the most effective strategies to reduce the risk of alloimmunization or autoimmunization?}

\section{Summary of the Evidence}

The systematic review summarized more than 60 longitudinal and cross-sectional studies, involving more than 6,000 participants, in which alloimmunization or autoimmunization was described in adults and children with SCD undergoing transfusion. Rates of alloantibody formation ranged from 6 percent to 85 percent, while autoantibody formation ranged from 4 percent to 10 percent. These studies provide incidence and prevalence data only, and none compared the effectiveness of preventive strategies.

Most alloimmunization developed against erythrocyte antigens in the Rh blood system ( $\mathrm{D}, \mathrm{Cc}, \mathrm{Ee})$ and other minor blood groups (e.g., Kell, Kidd, Duffy). Phenotype matching of these antigens between transfusion donor and recipient may lower the alloimmunization rate, with a reported rate of $0-7$ percent described in studies where strict matching criteria were employed. ${ }^{408-411}$ 


\section{Iron Overload}

\section{Background}

Transfused erythrocytes, whether administered through sporadic or repeated procedures, present an iron load to the recipient. The vast majority of the iron is carried by hemoglobin within the erythrocytes. As a rough calculation, 1 milliliter of erythrocytes contains approximately 1 milligram of iron, so for every 3-4 units of packed erythrocytes, 1 gram of iron enters the body. This process is clinically relevant, because adults normally have a total of only 4-5 grams of iron in their entire body, so this amount increases quickly after repeated transfusions. More importantly, there is no physiologic means to remove excess iron. Regulation of iron homeostasis normally occurs at the level of absorption through the hormone hepcidin, which inhibits the transport of gastrointestinal iron into the body. Because transfused blood represents iron that circumvents the normal pathways of iron regulation, this excess iron accumulates in tissues and can become pathological.

Hemosiderosis is a condition that reflects a large iron burden affecting normal organ function. The liver, pancreas, and heart are particularly vulnerable to iron overload. Chelation therapy can be used to remove excess iron. A number of different medications are used for chelation, but a thorough review of chelation dosing and management is beyond the scope of these guidelines. Deferoxamine is given by subcutaneous or intravenous route and leads to iron excretion through both urine and feces, whereas deferasirox is given orally once a day and removes iron primarily through the gastrointestinal tract. Deferiprone is taken orally three times a day and requires close monitoring due to the risk of agranulocytosis. Patients on monthly chronic transfusions typically receive chelation therapy to reduce iron burden, to attempt to normalize iron stores, and to avoid organ damage from hemosiderosis. ${ }^{408,412}$

\section{Diagnostic Tools for Assessing Iron Overload}

Changes in serum ferritin (SF) roughly correlate with iron loading, but the relationship is too inaccurate to use as a reliable method for evaluating iron status. Rather, SF is used as a biomarker to track qualitative trends of iron loading and chelation efficacy over time. Liver biopsy has been the gold standard in the diagnosis of iron overload but carries procedural risks and the possibility of sampling error. To avoid this invasive procedure, new diagnostic tools using MRI have been developed; these tools image the whole organ to quantify liver iron. Data are limited on the sensitivity and specificity of these new technologies to quantify liver iron in individuals with SCD. However, a significant body of literature supports the use of MRI as a substitute for liver biopsy for diagnosing iron overload in individuals with thalassemia. ${ }^{413}$ There is no reason to believe that the quantification of tissue iron would be different in individuals with SCD, and there is literature where MRI was used as (1) a screening tool for identifying patients eligible to participate in a trial of chelation therapy ( 80 patients; Cappellini et al. $\left.2010^{414}\right)$, (2) a tool to monitor outcomes in a study of chronically transfused SCD patients (15 patients; Hernandez et al. $1988^{232}$ ), or (3) a tool in studies examining different chelation regimens (15 patients;

Voskaridou et al. 2005; Cancado et al. 2009; ${ }^{415,416}$ Levin et al. 1995; Ghoti et al. 2010 ${ }^{417,418}$ ). Therefore, the expert panel considered the results in thalassemia patients when making recommendations for individuals with SCD.

\section{Key Questions}

KQ28. In patients with SCD undergoing chronic transfusion therapy, what are the effective strategies to reduce iron overload, and what are the most accurate diagnostic tests to estimate iron overload? 


\section{Summary of the Evidence}

A total of 50 studies ( 2 RCTs, 35 observational, and 13 cross-sectional) plus 9 case reports related to transfusion-acquired iron overload were identified. One $\mathrm{RCT}^{419}$ compared the use of deferasirox (oral) to deferoxamine (subcutaneous injections) in adults and children. The trial included 195 patients who were all iron overloaded (SF of at least $1,000 \mathrm{ng} / \mathrm{mL}$, along with liver iron content of at least $2 \mathrm{mg}$ iron $/ \mathrm{g}$ dry weight of liver tissue in patients receiving simple transfusions, and $5 \mathrm{mg}$ iron $/ \mathrm{g}$ dry weight of liver tissue in patients receiving exchange transfusions) and demonstrated that the two approaches yielded similar results. The second RCT was the STOP trial, ${ }^{408}$ which did not evaluate treatments for iron overload; however, enrolled children in this trial received chronic transfusion, which was associated with a rise in SF in the first year of the trial and which necessitated treatment with deferoxamine in several children. Twenty other observational studies compared different chelation agents, and all have consistently demonstrated reduction of iron overload as measured by several methods. Data regarding the comparison among the different chelating agents or against alternative approaches such as hydroxyurea and exchange transfusion are unavailable or of very low quality.

Most studies used an SF level $>1,000 \mathrm{ng} / \mathrm{mL}$ to diagnose patients with possible iron overload (often an inclusion criterion in the study). However, some studies used cutoffs of $1,500 \mathrm{ng} / \mathrm{mL}$ or higher. ${ }^{420,421}$ SF changes were nonlinear. Levels less than $1,500 \mathrm{ng} / \mathrm{mL}$ indicated mostly acceptable iron overload; levels of 3,000 ng/mL or greater were specific for significant iron overload and were associated with liver injury. Using a cutoff of $2,500 \mathrm{ng} / \mathrm{mL}$, Karam et al. ${ }^{412}$ reported that SF had sensitivity of 62.5 percent and specificity of 77.8 percent for identifying liver iron concentrations of $7 \mathrm{mg}$ iron/g dry liver tissue or greater. One observational study defined iron overload by liver iron concentration of at least $2.2 \mathrm{mg}$ iron/g dry weight of liver tissue. ${ }^{422}$ Sufficient data were not found to allow the estimation of diagnostic accuracy of MRI, although many chelation studies used MRI findings as inclusion criteria.

\section{Hemolysis}

\section{Background}

Hemolysis (the breakdown and destruction of donor erythrocytes) can occur during or after a transfusion. It is important to note that the mechanism of transfusion-related hemolysis is immunologic, in contrast to the hemolysis of sickled erythrocytes, which is an intrinsic red cell defect. Most transfusion-associated hemolysis occurs 1 to 4 weeks after red cell transfusion and is called a delayed hemolytic transfusion reaction (DHTR). DHTR is related to immune-mediated mechanisms. The most common pathophysiology is preexisting or new IgG alloantibodies that bind to the erythrocytes and lead to accelerated clearance by macrophages in the extravascular compartment within the spleen, liver, marrow, and other parts of the reticuloendothelial system (RES). If the antibodies also fix complement, then erythrocyte destruction is further accelerated through lysis directly within the intravascular compartment. Both extravascular and intravascular hemolysis are manifest by shortened red blood cell survival, worsening anemia, and increased titers of antibodies found either on the erythrocytes themselves (positive direct antiglobulin, or "Coombs" test) or in the serum (positive indirect antiglobulin test) after the transfusion.

DHTRs can be associated with hyperhemolysis or bystander hemolysis. In this life-threatening complication of transfusion, patients will hemolyze not only the transfused blood but also their own RBCs, causing a profound anemia. This complication is recognized when the hemoglobin falls below pretransfusion levels and is often associated with reticulocytopenia and a positive direct Coombs test suggesting autoimmune destruction of RBCs. ${ }^{423,424}$ 
Clinicians should have a high index of suspicion for hemolysis after transfusions, and they should coordinate diagnostic testing with the appropriate blood bank or transfusion service. Avoidance of future hemolytic events depends on proper diagnostic testing and avoidance of offending erythrocyte antigens.

\section{Key Question}

KQ29. In patients with SCD undergoing transfusion therapy, what are the most effective strategies to reduce the risk of hemolysis?

\section{Summary of the Evidence}

Three RCTs, 17 observational studies, and 47 case reports were identified related to hemolysis in association with transfusions. The RCTs included more than 300 patients. ${ }^{406,425,426}$ The studies described a prevalence of hemolytic reactions that ranged from 2 percent to 25 percent and an incidence of hyperhemolysis of 6 percent. There were no studies providing comparative effectiveness data on therapy. Descriptive studies reported successful management of the DHTR/hyperhemolysis (DHTR/H) syndrome with steroids, erythropoietin, and transfusion of phenotypically matched RBCs. The quality of evidence for management of these complications in SCD is very low, and data from transfusion in other populations may be indirectly applicable.

\section{Hyperviscosity}

\section{Background}

Transfusion of erythrocytes will increase the hematocrit of circulating blood, and increased viscosity could be problematic for patients with SCD. Avoidance of hyperviscosity is an important goal to prevent triggering a VOC.

\section{Key Question}

KQ30. In patients with SCD undergoing transfusion therapy, what are the most effective strategies to prevent and treat transfusion-associated hyperviscosity?

\section{Summary of the Evidence}

No studies were found that described the effectiveness of a particular preventive or therapeutic approach for hyperviscosity in SCD.

\section{Recommendations for the Management and Prevention of Transfusion Complications}

Although the literature summarized and described in the evidence table is of very low quality in most of the areas relating to transfusion complications, the expert panel opted to provide several recommendations based on extrapolation from transfusion literature on non-SCD populations, in vitro data, and the clinical expertise of the panel members. The panel members felt that clinicians in the field needed guidance to manage transfusion complications in patients with SCD and a comprehensive overview of this management. 


\section{Recommendations for Both Children and Adults}

1. Obtain patient transfusion history to include locations of prior transfusions and adverse effects. (Consensus-Panel Expertise)

2. Ask the blood bank to contact hospitals where patient reported receiving previous transfusion therapy to obtain transfusion information.

(Consensus-Panel Expertise)

3. RBC units that are to be transfused to individuals with $S C D$ should include matching for $C, E$, and $K$ antigens. (Moderate Recommendation, Low-Quality Evidence)

4. Consult the blood bank for a workup of a possible DHTR in a patient with any of the following signs or symptoms: acute anemia, pain, or jaundice within 3 weeks after a blood transfusion.

(Strong Recommendation, Moderate-Quality Evidence)

5. In patients with SCA who are not chronically transfused and who are therefore at risk for hyperviscosity, avoid transfusing to a target hemoglobin above $10 \mathrm{~g} / \mathrm{dL}$ (unless the patients are already on chronic transfusions or have low percent HbS levels).

(Moderate Recommendation, Low-Quality Evidence)

6. In patients who receive chronic transfusion therapy, perform serial assessment of iron overload to include validated liver iron quantification methods such as liver biopsy, or MRI R2 or MRI T2* and R2* techniques. The optimal frequency of assessment has not been established and will be based in part on the individual patient's characteristics. (Strong Recommendation, Moderate-Quality Evidence)

7. Administer iron chelation therapy, in consultation with a hematologist, to patients with SCD and with documented transfusion-acquired iron overload.

(Moderate Recommendation, Moderate-Quality Evidence) 


\section{Chapter 7: Looking Forward}

The process of developing guidelines for the management of persons with SCD has been challenging, as highquality evidence is limited in virtually every area related to SCD management. The systematic review of the literature identified a very small number of RCTs in individuals with SCD (for example, only three evaluating hydroxyurea, one of the most promising treatments), clearly demonstrating the extensive knowledge gaps in SCD and care of individuals with SCD.

\section{New Research Is Needed}

Cure is always the most desirable outcome for any chronic disease. Therefore, research that increases the evidence for and availability of a cure for SCD is a high priority. Hematopoietic stem cell transplantation (HSCT, formerly called bone marrow transplantation) is a treatment option for an increasing but still small number of people with SCD. The procedure involves "conditioning" therapy, utilizing myelosuppressive and/or immune-modifying drugs, followed by infusion of histocompatible stem cells (derived from bone marrow, peripheral blood, or umbilical cord blood). Substantial risks are involved with the procedure, and it is not yet feasible in the majority of people with SCD. Although clinical trials have provided promising results, and cure appears to be possible in a large proportion of patients receiving HSCT, ${ }^{427}$ additional research is still needed that addresses the potential risks of this therapy (e.g., failure of engraftment and chronic graft-versus-host disease) before HSCT can become a widely used therapy. ${ }^{428}$

Additional research is also required to address the many other areas with little or no evidence that were identified during the development and writing of these guidelines. The needed studies include observational work to better describe the utility of screening asymptomatic individuals with SCD for commonly occurring chronic diseases; studies to better describe the clinical course of the occurrence and treatment results of all the acute and chronic complications of SCD; comparative effectiveness studies to provide clear outcomes on best approaches to SCD and its complications; clinical trials for new therapeutic approaches or to improve on current therapeutic approaches such as examining the role of hydroxyurea in people with genotypes and clinical manifestations other than those in the MSH study or transfusion goals in chronic conditions. A few of the other larger research agenda issues, in addition to the need for an SCD cure, are summarized below.

\section{Data Systems That Meet the Highest Standards of Scientific Rigor Can Be Invaluable Resources}

Well-designed databases with linked biorepositories are required to complete observational studies and enable investigators to generate and test specific hypotheses. The databases must include longitudinal information on patient outcomes, therapies, health care services received, and the health system context in which the services were provided. The patient cohorts must be well-characterized by genotypic and phenotypic data. For example, this might be facilitated by mobilizing the Nation's diverse newborn hemoglobinopathy screening programs to pool and analyze their data. Some of these State-supported and academic center-based programs already have assembled valuable information on treatment and long-term outcomes of individuals identified as having SCD, which could be leveraged for the longitudinal studies. 


\section{Improved Phenotyping Is Needed}

The new expanded databases and data systems should enable the development of clinical and perhaps health care resource utilization phenotypes of individuals with SCD. Phenotyping includes the characterization of specific clinical, laboratory, imaging, and health care utilization features unique to some but not all individuals with SCD. Phenotypes require development of specific terminology to make such phenotyping as precise as possible. Phenotyping will require expanded collaboration among specialists in multiple disciplines to aggressively participate in and lead SCD-related research.

\section{Broad Collaborations for Research and Care}

SCD is a chronic condition that impacts every part of a person's body and most aspects of their daily life. Research regarding such conditions requires broad-based expertise. This process has begun to take shape during the past decade, with neurologists, pulmonologists, behavioral scientists, and health services researchers using their unique training and tools to improve SCD management. The communities of pain specialists, epidemiologists, informatics experts, and basic and translational scientists should come together to develop the framework for future management guidelines for SCD.

\section{Beyond Efficacy: From Bench to Bedside and the Community}

Perhaps the largest need is to translate the results of research that has been performed in laboratories and academic centers into community-based practice tools and clinical protocols. Such translational studies should lead to effectiveness trials. "Efficacy" does not always translate to "effectiveness." One of the best examples is hydroxyurea. Although hydroxyurea has proven efficacious in RCTs, the majority of eligible persons with SCA in the United States do not yet receive this agent. Well-designed effectiveness and translational studies are needed to overcome the identified barriers that result in underutilization of hydroxyurea. These barriers include the limited number of physicians with knowledge and experience with the agent, and patients' misconceptions and fears about side effects. Studies are also needed to examine the role of hydroxyurea in people with genotypes and clinical manifestations other than those in the MSH study. ${ }^{9}$ The SCD research community can make a real difference through comparative effectiveness research and other investigative strategies, as well as strengthening clinical and public health SCD collaborative efforts at the State and local levels. Finally, all of the research addressed in these guidelines cannot be successful for the approximately 70,000 to 100,000 individuals with SCD in the United States until sickle cell centers, practicing hematologists, and primary care providers in particular are fully willing and capable of taking on the challenges of serving these individuals and their families.

The expert panel realizes that these guidelines leave many uncertainties for health professionals caring for or planning to begin caring for individuals with SCD. However, we hope that these guidelines begin to facilitate improved and more accessible care for all individuals with SCD, and that the discrepancies in the data will trigger new research programs and processes that will provide the evidence necessary to expand upon evidencebased SCD guidelines in the future. 


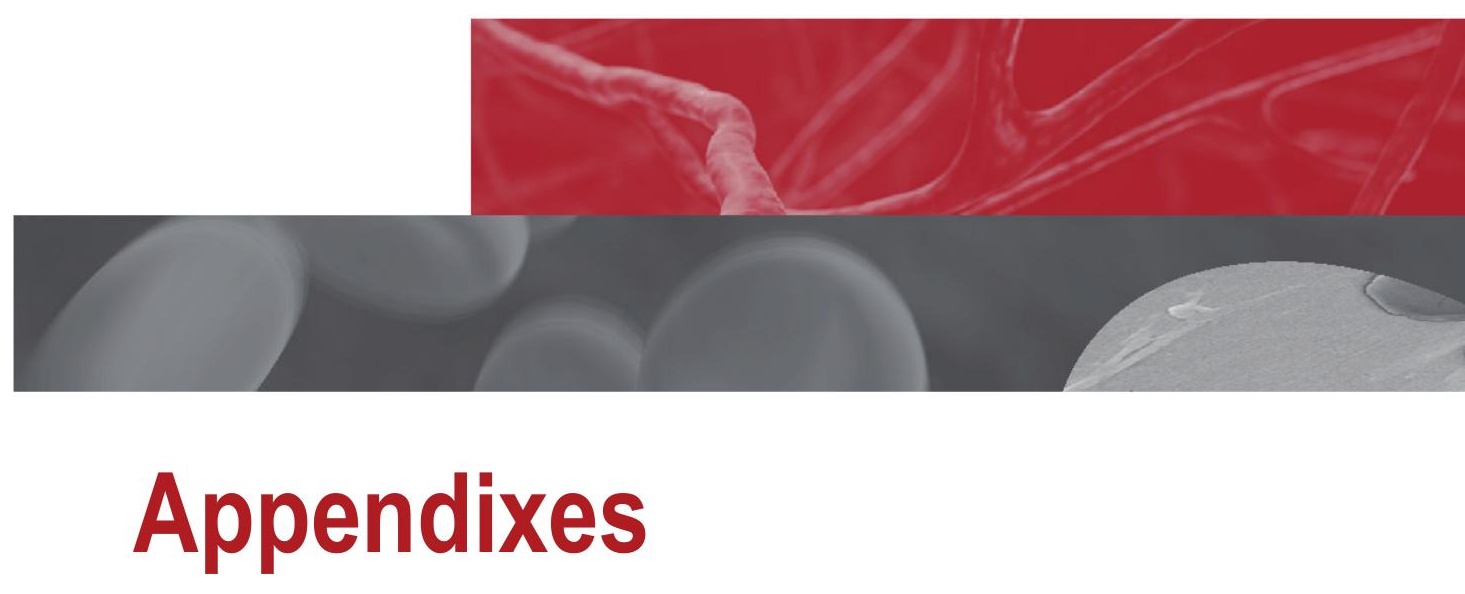





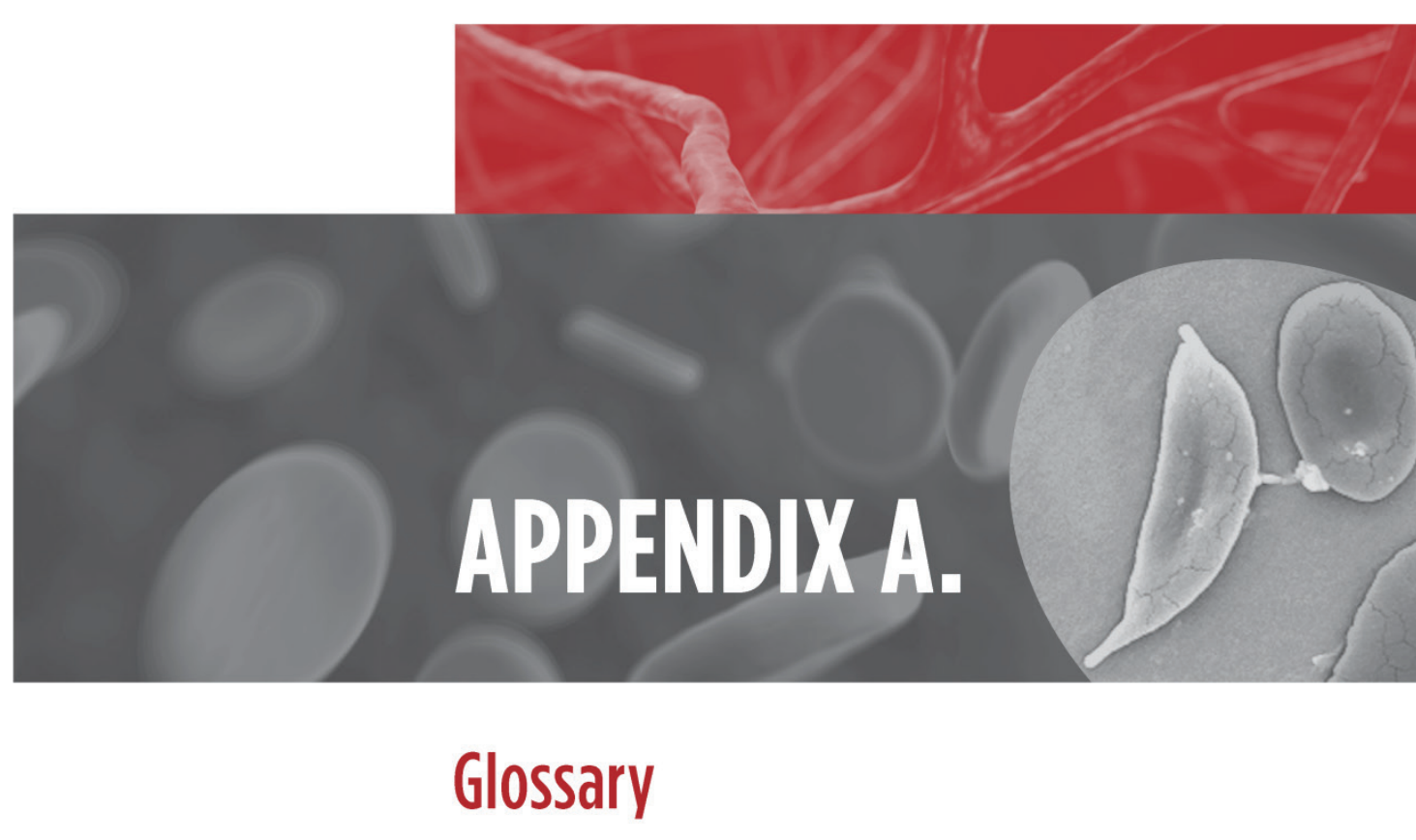





\section{Appendix A. Glossary}

5-azacytidine

Acute chest syndrome (ACS)

Acute hepatic sequestration (AHS)

Acute intrahepatic cholestasis (AIC)

$\alpha$-gene deletion

Alloimmunization

Anterior chamber of eye

Autoimmunization

Avascular necrosis

Azotemia

CAR $\beta$-haplotype
An analog of the pyrimidine nucleoside cytidine. It is used primarily in the treatment of acute myeloid leukemia and myelodysplastic syndrome. It was also found to raise fetal hemoglobin levels but is not approved for use in the care of individuals with sickle cell disease.

An acute illness characterized by fever and/or respiratory signs and symptoms, accompanied by a new pulmonary infiltrate involving at least one complete lung segment consistent with the presence of alveolar consolidation on a chest $\mathrm{x}$ ray.

Liver enlargement below the right costal margin of greater than or equal to $3 \mathrm{~cm}$ for children and greater than or equal to $5 \mathrm{~cm}$ for adults from previous physical exam without other explanation and a $2 \mathrm{~g} / \mathrm{dL}$ or greater drop in hemoglobin level over a few hours to days.

Rapidly developing interruption in the excretion of bile caused by obstruction within the liver associated with severe abnormalities of liver function tests and coagulation parameters.

Lack of one or more of the four alpha globin genes on chromosome 16.

An immunological response by the recipient against "foreign" non-self-antigens that may follow an erythrocyte transfusion and result in destruction of transfused erythrocytes.

The space between the cornea and the iris containing aqueous fluid.

In transfusion medicine, the term refers to the development of an immune response to an individual's own erythrocytes, which may result in the destruction of erythrocytes.

Bone death due to compromised blood supply of the bone.

An elevation of blood urea nitrogen (BUN) and serum creatinine above normal levels.

A variant of the beta chain of the sickle hemoglobin that is prevalent in the Central African Republic (CAR). Also known as Bantu (Ban) haplotype. 
Case fatality rate

Cellular rheology

Central retinal artery occlusion (CRAO)

Central sensitization

Cholangitis

Choledocholithiasis

Cholestatic jaundice

Chronic kidney disease (CKD)

Chronic sickle cell pain

Dactylitis

Delayed hemolytic transfusion reaction (DHTR)

Disease-modifying therapies

Erythrocytapheresis

Exchange transfusion

Fix complement
Percentage of persons diagnosed as having a specified disease who die as a result of that illness within a given time period.

In the case of erythrocytes, this term refers to the flow dynamics of red blood cells and their ability to negotiate microvasculature due to their elastic and plastic properties.

Blockage of the retinal artery.

An event that follows repetitive painful stimuli and sensitizes the central nervous system so that it perceives innocuous stimuli as painful.

A severe infection of the bile ducts.

The presence of gallstones in the common bile duct.

Jaundice of the skin and/or sclera due to dysfunction of the hepatobiliary system.

Either having a glomerular filtration rate (GFR) of less than $60 \mathrm{~mL} / \mathrm{min} / 1.73 \mathrm{~m}^{2}$ for greater than or equal to 3 months with or without kidney damage or having evidence of kidney damage for greater than or equal to 3 months, with or without decreased GFR, manifested by either pathologic abnormalities or markers of kidney damage (i.e., proteinuria) independent of cause.

Pain that does not resolve and lasts for more than 3 months.

A vaso-occlusive crisis involving one or often multiple small bones, and characterized by swelling and pain in the hands and/or feet, occurring in infants or young children.

Hemolysis of donor erythrocytes 1-4 weeks after a transfusion, due to the development of alloantibodies by the recipient toward the donor erythrocyte.

Treatments or drugs that impact the course of a disease by slowing the progression of the disease and decreasing the number of relapses.

Removal of recipient erythrocytes prior to and/or during donor erythrocyte infusion. This requires the use of an apheresis device.

Removal of recipient blood prior to and/or during donor erythrocyte infusion. This can be accomplished by erythrocytapheresis or by a manual method.

In transfusion medicine, this term refers to antigenantibody complexes binding complement, leading to complement-mediated lysis of erythrocytes. 
Fluorescein angiography

$\mathrm{FEV}_{1}$

FVC

$\mathrm{FEV}_{1} / \mathrm{FVC}$ Percent (\%)

Glomerular filtration rate (GFR)

$\mathrm{Hb}$

$\mathrm{HbA}$

$\mathrm{HbAS}$

$\mathrm{HbF}$

$\mathrm{HbS}$

HBS $\alpha$-Thal

$\mathrm{HbSC}$

$\mathrm{HbSS}$

Hemoglobinopathy

HLA

Hydroxyurea

Hyperhemolysis posttransfusion
An eye test that uses a fluorescein dye and camera to examine the circulation in the retina and choroid.

Forced expiratory volume in 1 second. The amount of air which can be forcibly exhaled from the lungs in the first second of an exhalation. Usually reported as both liters and percent predicted comparing to people of the same age, gender, and height.

Forced vital capacity. The amount of air which can be forcibly exhaled from the lungs after taking the deepest breath possible. Usually reported as both liters and percent predicted comparing people to similar age, gender, and height.

The ratio of $\mathrm{FEV}_{1}$ to $\mathrm{FVC}$, which tells the clinician what percentage of the total amount of air is exhaled from the lungs during the first second of forced exhalation. This is considered a clinical measure of obstructive lung disease.

The total of filtration rates of all functional kidney nephrons. The GFR is estimated by measuring markers such as creatinine and Cystatin $\mathrm{C}$.

Hemoglobin.

Hemoglobin A, normal hemoglobin.

Hemoglobin A plus sickle hemoglobin; the carrier state for sickle cell anemia, also known as sickle cell trait.

Fetal hemoglobin.

Sickle hemoglobin.

Hemoglobin SS $+\alpha$ thalassemia.

Sickle cell hemoglobin C disease.

Homozygous sickle cell disease.

A disorder characterized by an abnormality of the structure or function of hemoglobin.

Human Leukocyte Antigen system is the name of the major histocompatibility complex (MHC) in humans.

A ribonucleotide reductase inhibitor, initially used to treat patients who had myeloproliferative disorders; also known as hydroxycarbamide.

A drop in hematocrit (hemoglobin concentration) below pretransfusion levels after transfusion. Often associated with reticulocytopenia and identification of alloantibodies. 
Hypersplenism

Hyperviscosity

Hyphema

Hyposthenuria

Indirect Coombs test

Interconception period

Iontophoresis

Leukocyte reduced

Macroalbuminuria

Mean corpuscular volume (MCV)

Microalbuminuria

Moyamoya syndrome

Neuropathic pain

Neuroplasticity

Orbital compression syndrome (OCS)

Pain management protocol
Enlargement of the spleen associated with reduction in multiple blood cell types.

An increase in the resistance of blood to flow through vessels. This can occur due to an increase hemoglobin concentration of circulating blood, which could trigger vaso-occlusion.

Blood in the anterior chamber of the eye.

The inability to concentrate urine.

Blood bank test used to identify alloantibodies in serum produced in response to exposure to foreign non-self red blood cell antigens; also known as indirect antiglobulin test (IAT).

An 18- to 24-month interval between the birth of one child and the conception of the next.

The introduction of an ionized substance (as a drug) through intact skin by the application of a direct electric current.

Donor erythrocytes that are filtered to reduce the number of white blood cells.

Urinary excretion of albumin typically greater than 300 milligrams per 24 hours.

The average volume of red blood cells measured in femtoliters (fL).

Urinary excretion of albumin, typically between 30 and 300 milligrams per 24 hours.

A rare progressive cerebrovascular disorder caused by blocked arteries at the base of the brain in the basal ganglia.

Pain caused by a lesion or disease of the central or peripheral somatosensory system.

The ability of the central nervous system to change and adapt to new experiences.

Marked swelling around the eye associated with pain and visual disturbances resulting from avascular necrosis of the orbital bone.

A detailed written plan that provides guidance for dosing of analgesic agents to achieve pain management. Protocols can be written for an individual patient by the clinician who provides care and best understands analgesic needs during a vaso-occlusive crisis (VOC), or they can be 
Partial exchange transfusion

Patient-controlled analgesia (PCA)

PEFR

Posterior chamber of eye

Preimplantation genetic diagnosis (PGD)

Priapism

Primary care provider

Proliferative sickle retinopathy (PSR)

Proteinuria

Pulmonary arterial hypertension (PAH)

Sickle cell anemia developed as a more generic protocol, specific to patients with SCD, often with higher analgesic doses and more frequent dosing intervals. Protocols should be based upon the individual's pain score and analgesic history.

Removal of a volume of recipient blood less than the total blood volume and replacement with donor erythrocytes.

A drug-delivery system that uses an intravenous pump to dispense a preset dose of a narcotic analgesic when the patient pushes a button. The PCA dose allows the patient to administer a dose up to every 10 minutes if needed. Additional basal or continuous background infusions may or may not be required based upon the individual, analgesic history, and current needs. Orders are written to define the PCA dose administered and PCA time interval every time the patient presses the PCA button. A lock out, or maximum dose per hour, is also written as a part of the PCA orders.

Peak expiratory flow rate. A measurement of how fast a person can exhale.

The space behind the iris and in front of the lens filled with aqueous humor.

Testing performed on an embryo before it is transferred to the uterus to determine if it also carries a genetic abnormality when one or both genetic parents have a known genetic abnormality.

A sustained, unwanted, painful penile erection lasting 4 or more hours.

Internist, family physician, pediatrician, nurse practitioner, or physician assistant with a clinical focus on the provision of general health care for the patient.

Growth of new vessels that emerge from the retinal vasculature at the interface of perfused and nonperfused retina in response to vascular growth factors produced by retinal ischemia. Also known as sea fan.

Any urinary protein excretion greater than normal (less than $300 \mathrm{mg} /$ day).

An elevation of pulmonary arterial systolic pressure (PASP) (greater than $25 \mathrm{mmHg}$ at rest or greater than 30 $\mathrm{mmHg}$ with exercise) determined by right heart catheterization.

Genotypes $\mathrm{HbSS}$ and $\mathrm{HbS} \beta^{0}$-thalassemia, which are associated with the most severe clinical manifestations, are commonly referred to as sickle cell anemia. 
Sickle cell disease (SCD)

Sickle vasculopathy

Simple transfusion

Stuttering priapism

Transcranial Doppler ultrasonography (TCD)

Tricuspid valve regurgitant jet velocity (TRV or TRJ)

Urobilinogenuria

Vaso-occlusive crisis (VOC)
Sickle cell disease (SCD) is caused by inherited mutations involving the beta globin gene that result in the formation of an abnormal hemoglobin (hemoglobin S). Red blood cells, which contain a predominance of hemoglobin $\mathrm{S}$, undergo shape change when low oxygen concentrations cause polymerization of the sickle hemoglobin. The damaged red blood cells become rigid and inflexible, occluding blood vessels and inducing tissue ischemia, pain, and organ damage. This process is accompanied by an inflammatory response and shortened red blood cell survival. These alterations may result in a wide variety of clinical manifestations.

SCD genotypes (exhibit 1) include homozygosity of the sickle hemoglobin gene (HbSS) and the compound heterozygous conditions hemoglobin $\mathrm{S} \beta^{0}$-thalassemia $\left(\mathrm{HbS} \beta^{0}\right)$, hemoglobin $\mathrm{S} \beta^{+}$-thalassemia $\left(\mathrm{HbS} \beta^{+}\right)$, hemoglobin $\mathrm{SC}$ disease (HbSC), and other, much less prevalent combinations. HbSS, the most prevalent genotype, and $\mathrm{HbS} \beta^{0}$ are commonly referred to as sickle cell anemia (SCA) and are associated with the most severe clinical manifestations.

Multiple biological processes contribute to the pathogenesis of vasculopathy, including red cell sickling, inflammation and adhesion biology, coagulation activation, stasis, deficient bioavailability and excessive consumption of nitric oxide, excessive oxidation, and reperfusion injury physiology. This leads to abnormal vascular tone and activated, adhesive endothelium.

The infusion of donor erythrocytes without removal of recipient blood.

Multiple self-limited episodes of priapism, each lasting less than 4 hours.

A noninvasive method of analyzing blood flow velocity in the brain.

Transthoracic echocardiographic determination of pulmonary hypertension. Elevated pulmonary artery pressure results require confirmation by right heart catheterization.

The presence of urobilinogen (a metabolite of bilirubin) in the urine.

Pain resulting from tissue ischemia as a result of blockage of blood vessels, occurring in a variety of vascular beds, but most commonly in the bone or bone marrow and requiring analgesic medication. Also known as sickle crisis, acute pain crisis, or vaso-occlusive episodes. 
Vital capacity

Vitreous chamber of eye

Vitreoretinal traction forces
The volume of air that can be expelled from the lungs from a position of full inspiration, with no limit to duration of inspiration; equal to inspiratory capacity plus expiratory reserve volume.

The vitreous chamber occupies the posterior $4 / 5$ ths of the eye. It consists of the space between the lens and the retina, and is filled with a transparent gel called the vitreous humor.

Forces in the vitreous chamber of the eye due to trauma or the proliferation of the new fibrosed vessels that exert negative pressure on the retina, which, if severe, may cause retinal detachment. 



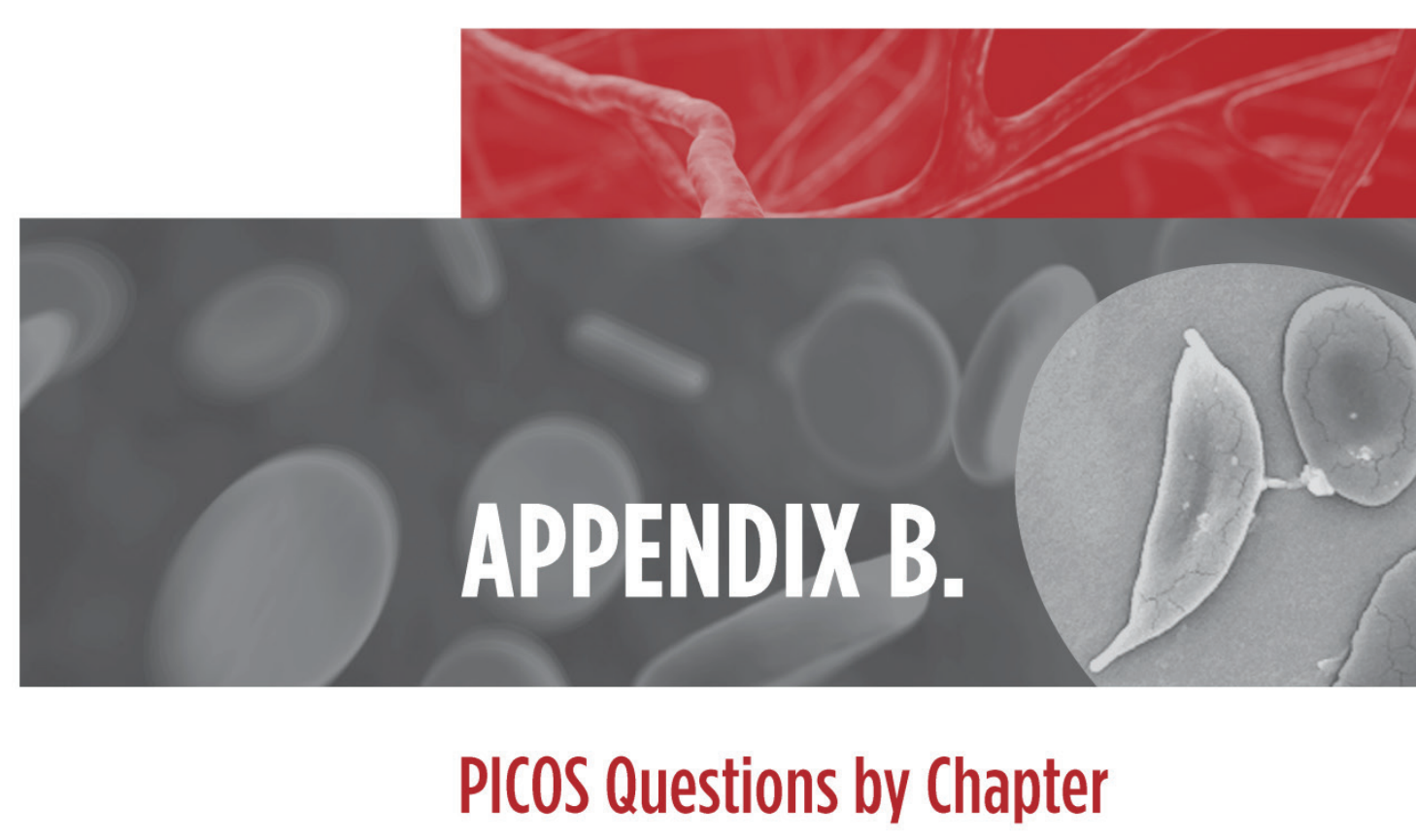





\section{Appendix B. PICOS Questions by Chapter}

\section{Exhibit B-1. PICOS Approach for Health Maintenance Chapter: Antibiotics}

\section{Patients}

Intervention

Comparison

Outcomes

Outcomes

Study Design

\section{Children With SCD}

Prophylactic antibiotic therapy

Placebo, no prophylactic antibiotic therapy

Incidence of relevant infections

Incidence of relevant mortality

Incidence of adverse effects of prophylactic antibiotics

Randomized or nonrandomized studies with original data

\section{Exhibit B-2. PICOS Approach for Health Maintenance Chapter: Screening}

\section{Patients}

Individuals With SCD

\begin{tabular}{|c|c|}
\hline Intervention & $\begin{array}{ll}\text { Screening strategies using the following tests: } \\
\text { - } & \text { Electrocardiogram } \\
\text { - } & \text { Echocardiogram } \\
\text { - } & \text { Pulmonary function testing } \\
\text { - } & \text { Renal function testing } \\
\text { - } & \text { Serial eye/retinal examination } \\
\text { - } & \text { Brain imaging } \\
\text { - } & \text { Transcranial Doppler }\end{array}$ \\
\hline Comparison & Control group in which no screening was provided \\
\hline $\begin{array}{l}\text { Alternate Study } \\
\text { Type }\end{array}$ & $\begin{array}{l}\text { Studies that compared patients identified by screening in an asymptomatic state; then those with } \\
\text { positive screening tests were subjected to treatment vs. no treatment }\end{array}$ \\
\hline Outcomes & Development of acute and chronic complications \\
\hline Study Design & Randomized or nonrandomized (including noncontrolled studies) \\
\hline
\end{tabular}


Exhibit B-3a. PICOS Approach for Health Maintenance Chapter: Blood Pressure (Question 1)

\begin{tabular}{|l|l|}
\hline Patients/Exposure & \multicolumn{1}{c|}{ Individuals With SCD } \\
\hline Comparison & Age- or sex-matched healthy controls or patients with confirmed AA genotype \\
\hline Outcomes & - $\quad$ Diastolic, systolic, and mean blood pressure \\
& - Prognosis of hypertension \\
\hline Study Design & Randomized or nonrandomized studies with original data \\
\hline
\end{tabular}

\section{Exhibit B-3b. PICOS Approach for Health Maintenance Chapter: Blood Pressure (Question 2)}

\begin{tabular}{|l|l|}
\multicolumn{1}{|c|}{ Patients } & \multicolumn{2}{c|}{ Individuals With SCD and Hypertension } \\
\hline Comparison & Treatment of hypertension (pharmacological or lifestyle-based) \\
\hline Outcomes & - $\quad \begin{array}{c}\text { Cardiovascular and cerebrovascular outcomes } \\
\text { - Blood pressure control }\end{array}$ \\
\hline Study Design & Randomized or nonrandomized studies with original data \\
\hline
\end{tabular}

\section{Exhibit B-4. PICOS Approach for Acute and Chronic Complications Chapters}

\begin{tabular}{|l|l|}
\multicolumn{2}{|c|}{ Patients } \\
\begin{tabular}{|l|l|} 
Intervention/ \\
Comparison
\end{tabular} & Alternative management and diagnosis strategies \\
\hline Outcomes & $\begin{array}{l}\text { Complication-specific outcomes including resolution of complication: } \\
\text { General SCD outcomes if relevant (death, stroke, pain crises, need for transfusion, hemoglobin } \\
\text { and hemoglobin F levels) }\end{array}$ \\
\hline Study Design & Outcomes of diagnostic studies: Accuracy of diagnosis if reported \\
\hline
\end{tabular}




\section{Exhibit B-5. PICOS Approach for Hydroxyurea Chapter}

\begin{tabular}{|c|c|}
\hline Patients & Patients With SCD (for Hydroxyurea Harms, Studies in Non-SCD Patients Were Included) \\
\hline Intervention & Hydroxyurea \\
\hline Comparison & Usual care without hydroxyurea \\
\hline Outcomes & $\begin{array}{l}\text { Benefits of hydroxyurea: } \\
-\quad \text { Death } \\
- \text { Stroke } \\
- \text { Pain crises } \\
- \text { Need for transfusion } \\
- \text { Hemoglobin } \\
- \text { Hemoglobin F levels } \\
\text { - Harms of hydroxyurea (adverse effects) } \\
\text { - Barriers to implementation of hydroxyurea treatment and interventions to overcome barriers } \\
\text { - Treatment protocols and monitoring parameters }\end{array}$ \\
\hline Study Design & Randomized or nonrandomized design including case reports of rare complications \\
\hline
\end{tabular}

\section{Exhibit B-6. PICOS Approach for Transfusion Chapter}

\begin{tabular}{|c|c|}
\hline Patients & Patients With SCD \\
\hline Intervention & Acute or chronic transfusion \\
\hline Comparison & Alternative transfusion strategies or alternative management other than transfusion \\
\hline Outcomes & $\begin{array}{l}\text { - } \quad \text { Complication-specific outcomes including resolution of complication } \\
- \text { Death } \\
- \text { Stroke } \\
-\quad \text { Pain crises } \\
-\quad \text { Need for transfusion } \\
-\quad \text { Hemoglobin and hemoglobin F levels } \\
-\quad \text { Hemoglobin S concentration } \\
\text { - Outcomes of diagnostic studies: Accuracy of diagnosis if reported }\end{array}$ \\
\hline Study Design & Randomized or nonrandomized including case reports of rare complications \\
\hline
\end{tabular}




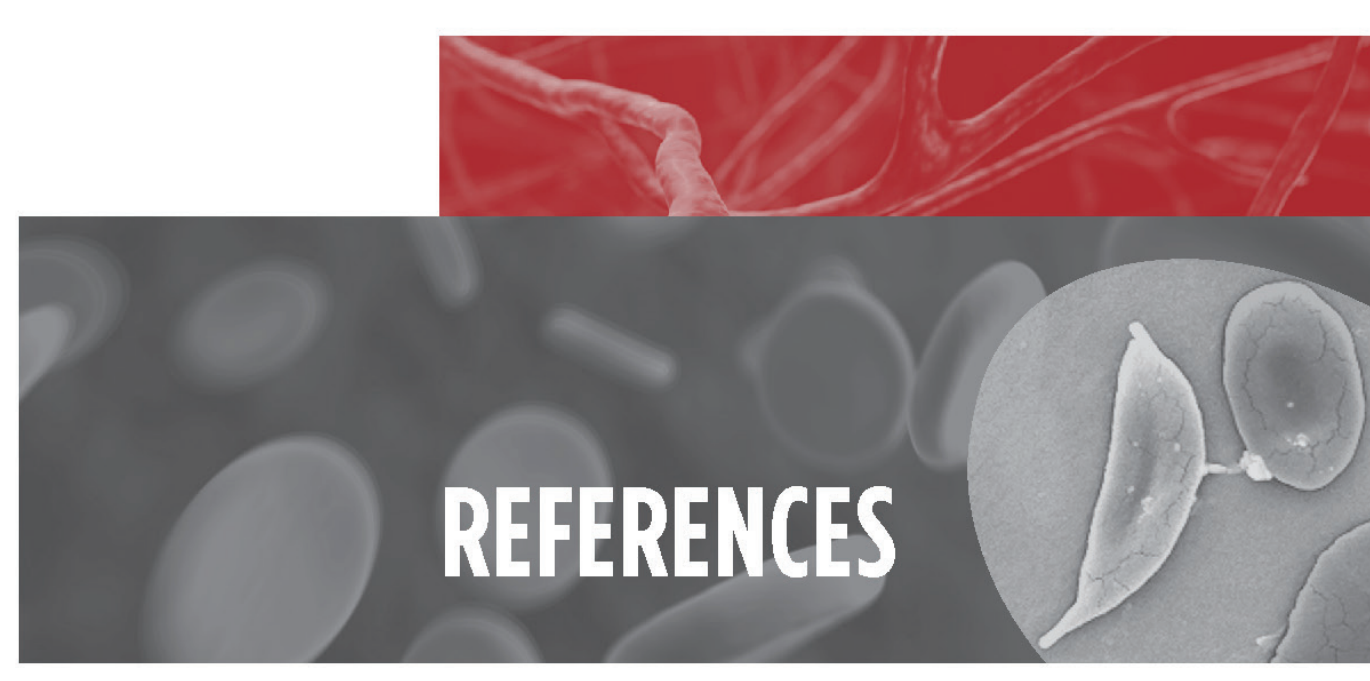




\section{References}

1. Herrick JB. Peculiar elongated and sickle-shaped red blood corpuscles in a case of severe anemia. Arch Intern Med. 1910;VI:517-21.

2. Hsieh MM, Fitzhugh CD, Tisdale JF. Allogeneic hematopoietic stem cell transplantation for sickle cell disease: the time is now. Blood. 2011;118(5):1197-207.

3. Hsieh MM, Kang EM, Fitzhugh CD, Link MB, Bolan CD, Kurlander R, et al. Allogeneic hematopoietic stem-cell transplantation for sickle cell disease. $N$ Engl J Med. 2009;361(24):2309-17.

4. Kamani NR, Walters MC, Carter S, Aquino V, Brochstein JA, Chaudhury S, et al. Unrelated donor cord blood transplantation for children with severe sickle cell disease: results of one cohort from the phase II study from the Blood and Marrow Transplant Clinical Trials Network (BMT CTN). Biol Blood Marrow Transplant. 2012;18(8):1265-72.

5. Locatelli F, Kabbara N, Ruggeri A, Ghavamzadeh A, Roberts I, Li CK, et al. Outcome of patients with hemoglobinopathies given either cord blood or bone marrow transplantation from an HLA-identical sibling. Blood. 2013;122(6):1072-8.

6. Lanzkron S, Strouse JJ, Wilson R, Beach MC, Haywood C, Park H, et al. Systematic review: hydroxyurea for the treatment of adults with sickle cell disease. Ann Intern Med. 2008;148(12):939-55.

7. Segal JB, Strouse JJ, Beach MC, Haywood C, Witkop C, Park H, et al. Hydroxyurea for the treatment of sickle cell disease. Evid Rep Technol Assess (Full Rep). 2008(165):1-95.

8. Strouse JJ, Lanzkron S, Beach MC, Haywood C, Park H, Witkop C, et al. Hydroxyurea for sickle cell disease: a systematic review for efficacy and toxicity in children. Pediatrics. 2008;122(6):1332-42.

9. Brawley OW, Cornelius LJ, Edwards LR, Gamble VN, Green BL, Inturrisi C, et al. National Institutes of Health Consensus Development Conference statement: hydroxyurea treatment for sickle cell disease. Ann Intern Med. 2008;148(12):932-8.

10. Dunbar LN, Coleman Brown L, Rivera DR, Hartzema AG, Lottenberg R. Transfusion practices in the management of sickle cell disease: a survey of Florida hematologists/oncologists. ISRN Hematol. 2012;2012:524513.

11. Wahl S, Quirolo KC. Current issues in blood transfusion for sickle cell disease. Curr Opin Pediatr. 2009;21(1):15-21.

12. Ingram VM. A specific chemical difference between the globins of normal human and sicklecell anaemia haemoglobin. Nature. 1956;178(4537):792-4.

13. Farrell K, Dent L, Nguyen ML, Buchowski M, Bhatt A, Aguinaga Mdel P. The relationship of oxygen transport and cardiac index for the prevention of sickle cell crises. J Natl Med Assoc. 2010;102(11):1000-7. 
14. Telfer P, Coen P, Chakravorty S, Wilkey O, Evans J, Newell H, et al. Clinical outcomes in children with sickle cell disease living in England: a neonatal cohort in East London.

Haematologica. 2007;92(7):905-12.

15. Quinn CT, Rogers ZR, McCavit TL, Buchanan GR. Improved survival of children and adolescents with sickle cell disease. Blood. 2010;115(17):3447-52.

16. Artz N, Whelan C, Feehan S. Caring for the adult with sickle cell disease: results of a multidisciplinary pilot program. J Natl Med Assoc. 2010;102(11):1009-16.

17. Platt OS, Brambilla DJ, Rosse WF, Milner PF, Castro O, Steinberg MH, et al. Mortality in sickle cell disease. Life expectancy and risk factors for early death. $N$ Engl J Med. 1994;330(23):1639-44.

18. Powars DR, Chan LS, Hiti A, Ramicone E, Johnson C. Outcome of sickle cell anemia: a 4decade observational study of 1056 patients. Medicine (Baltimore). 2005;84(6):363-76.

19. Wilson JMG, Jungner G. Principles and practice of screening for disease. Geneva, Switzerland: World Health Organization, 1968.

20. U.S. Preventive Services Task Force (USPTF). Recommendations [Internet]. Rockville, MD: USPTF; 2010 [updated December 2010; cited 2014 May 30]. Available from: http://www.uspreventiveservicestaskforce.org/recommendations.htm.

21. Centers for Disease Control and Prevention. U.S. Medical Eligibility Criteria for Contraceptive Use, 2010-Adapted from the World Health Organization Medical Eligibility Criteria for Contraceptive Use, 4th edition. MMWR Recomm Rep. 2010;59(RR-4):1-86.

22. Ahmed F, Temte JL, Campos-Outcalt D, Schunemann HJ; ACIP Evidence Based Recommendations Work Group (EBRWG). Methods for developing evidence-based recommendations by the Advisory Committee on Immunization Practices (ACIP) of the U.S. Centers for Disease Control and Prevention (CDC). Vaccine. 2011;29(49):9171-6.

23. Benjamin LJ, Dampier CD, Jacox A, Odesina V, Phoenix D, Shapiro BS, et al. Guideline for the management of acute and chronic pain in sickle-cell disease. Glenville, IL: APS Clinical Practice Guideline Series, No. 1, 1999.

24. Chou R, Fanciullo GJ, Fine PG, Adler JA, Ballantyne JC, Davies P, et al. Clinical guidelines for the use of chronic opioid therapy in chronic noncancer pain. J Pain. 2009;10(2):113-30.

25. Atkins D, Best D, Briss PA, Eccles M, Falck-Ytter Y, Flottorp S, et al. Grading quality of evidence and strength of recommendations. BMJ. 2004;328(7454):1490.

26. Gandhi GY, Murad MH, Fujiyoshi A, Mullan RJ, Flynn DN, Elamin MB, et al. Patientimportant outcomes in registered diabetes trials. J Am Med Assoc. 2008;299(21):2543-9.

27. Balshem H, Helfand M, Schunemann HJ, Oxman AD, Kunz R, Brozek J, et al. GRADE guidelines: 3. Rating the quality of evidence. J Clin Epidemiol. 2011;64(4):401-6. 
28. Guyatt GH, Oxman AD, Vist GE, Kunz R, Falck-Ytter Y, Alonso-Coello P, et al. GRADE: an emerging consensus on rating quality of evidence and strength of recommendations. $B M J$. 2008;336(7650):924-6.

29. Schunemann HJ, Jaeschke R, Cook DJ, Bria WF, El-Solh AA, Ernst A, et al. An official ATS statement: grading the quality of evidence and strength of recommendations in ATS guidelines and recommendations. Am J Respir Crit Care Med. 2006;174(5):605-14.

30. Shea BJ, Hamel C, Wells GA, Bouter LM, Kristjansson E, Grimshaw J, et al. AMSTAR is a reliable and valid measurement tool to assess the methodological quality of systematic reviews. J Clin Epidemiol. 2009;62(10):1013-20.

31. Kavanagh PL, Sprinz PG, Vinci SR, Bauchner H, Wang CJ. Management of children with sickle cell disease: a comprehensive review of the literature. Pediatrics. 2011;128(6):e1552-74.

32. Graham R, Mancher M, Wolman DM, Greenfield S, Steinberg E, editors. Clinical Practice Guidelines We Can Trust. Washington (DC): National Academies Press; 2011.

33. Stellefson M, Dipnarine K, Stopka C. The chronic care model and diabetes management in US primary care settings: a systematic review. Prev Chronic Dis. 2013;10:E26.

34. Gaston MH, Verter JI, Woods G, Pegelow C, Kelleher J, Presbury G, et al. Prophylaxis with oral penicillin in children with sickle cell anemia. A randomized trial. $N$ Engl J Med. 1986;314(25):1593-9.

35. Zarkowsky HS, Gallagher D, Gill FM, Wang WC, Falletta JM, Lande WM, et al. Bacteremia in sickle hemoglobinopathies. J Pediatr. 1986;109(4):579-85.

36. Falletta JM, Woods GM, Verter JI, Buchanan GR, Pegelow CH, Iyer RV, et al. Discontinuing penicillin prophylaxis in children with sickle cell anemia. Prophylactic Penicillin Study II. $J$ Pediatr. 1995;127(5):685-90.

37. John AB, Ramlal A, Jackson H, Maude GH, Sharma AW, Serjeant GR. Prevention of pneumococcal infection in children with homozygous sickle cell disease. Br Med J (Clin Res Ed). 1984;288(6430):1567-70.

38. Nkouwap I, Diara JP, Noyon I, Etienne-Julan M, Merault L. Is there any alternative to oral penicillin in antibioprophylaxis for children with sickle cell disease? [French] Y a-t-il une alternative a la penicilline orale dans l'antibioprophylaxie chez les enfants drepanocytaires? Med Mal Infect. 1999;29(2):111-6.

39. Guasch A, Cua M, Mitch WE. Early detection and the course of glomerular injury in patients with sickle cell anemia. Kidney Int. 1996;49(3):786-91.

40. Alvarez O, Montane B, Lopez G, Wilkinson J, Miller T. Early blood transfusions protect against microalbuminuria in children with sickle cell disease. Pediatr Blood Cancer. 2006;47(1):71-6.

41. Aluoch JR. Renal and electrolyte profile in steady state sickle cell disease: observations in patients with sickle cell disease in The Netherlands. Trop Geogr Med. 1989;41(2):128-32. 
42. Sklar AH, Campbell H, Caruana RJ, Lightfoot BO, Gaier JG, Milner P. A population study of renal function in sickle cell anemia. Int J Artif Organs. 1990;13(4):231-6.

43. Badesch DB, Champion HC, Sanchez MA, Hoeper MM, Loyd JE, Manes A, et al. Diagnosis and assessment of pulmonary arterial hypertension. J Am Coll Cardiol. 2009;54(1 Suppl):S5566.

44. Simonneau G, Robbins IM, Beghetti M, Channick RN, Delcroix M, Denton CP, et al. Updated clinical classification of pulmonary hypertension. J Am Coll Cardiol. 2009;54(1 Suppl):S43-54.

45. Barnett CF, Hsue PY, Machado RF. Pulmonary hypertension: an increasingly recognized complication of hereditary hemolytic anemias and HIV infection. JAMA. 2008;299(3):324-31.

46. Parent F, Bachir D, Inamo J, Lionnet F, Driss F, Loko G, et al. A hemodynamic study of pulmonary hypertension in sickle cell disease. N Engl J Med. 2011;365(1):44-53.

47. Machado RF, Barst RJ, Yovetich NA, Hassell KL, Kato GJ, Gordeuk VR, et al. Hospitalization for pain in patients with sickle cell disease treated with sildenafil for elevated TRV and low exercise capacity. Blood. 2011;118(4):855-64.

48. Sachdev V, Machado RF, Shizukuda Y, Rao YN, Sidenko S, Ernst I, et al. Diastolic dysfunction is an independent risk factor for death in patients with sickle cell disease. $\mathrm{J} \mathrm{Am}$ Coll Cardiol. 2007;49(4):472-9.

49. National Heart, Lung, and Blood Institute (US). What Is Pulmonary Hypertension? 2011 Apr 1 [cited 2013 Feb 19]. Available from: http://www.nhlbi.nih.gov/health/healthtopics/topics/pah/.

50. Fonseca GH, Souza R, Salemi VM, Jardim CV, Gualandro SF. Pulmonary hypertension diagnosed by right heart catheterisation in sickle cell disease. Eur Respir J. 2012;39(1):112-8.

51. Machado RF, Mack AK, Martyr S, Barnett C, Macarthur P, Sachdev V, et al. Severity of pulmonary hypertension during vaso-occlusive pain crisis and exercise in patients with sickle cell disease. Br J Haematol. 2007;136(2):319-25.

52. Bachir D, Parent F, Hajji L, Inamo J, Loko G, Lionnet F, et al. Prospective multicentric survey on pulmonary hypertension (PH) in adults with sickle cell disease. Conference start of the 51st Annual Meeting of the American Society of Hematology; Dec 5-8, 2009; New Orleans, LA: Blood; 2009.

53. Fonseca GH, Salemi VC, Gualandro DM, Jardim C, Sousa R, Gualandro SF. Diagnosis of pulmonary hypertension in adults with sickle cell disease. Eur Heart J. 2010;31:759.

54. Liem RI, Nevin MA, Prestridge A, Young LT, Thompson AA. Tricuspid regurgitant jet velocity elevation and its relationship to lung function in pediatric sickle cell disease. Pediatr Pulmonol. 2009;44(3):281-9.

55. Arslankoylu AE, Hallioglu O, Yilgor E, Duzovali O. Assessment of cardiac functions in sickle cell anemia with Doppler myocardial performance index. J Trop Pediatr. 2010;56(3):195-7. 
56. Klings ES, Machado RF, Barst RJ, Morris CR, Mubarek K, Gordeuk VR, et al. An Official ATS Clinical Practice Guideline: Diagnosis and treatment of pulmonary hypertension of sickle cell disease. Am J Respir Crit Care Med. 2014;189(6):727-40.

57. Gladwin MT, Sachdev V, Jison ML, Shizukuda Y, Plehn JF, Minter K, et al. Pulmonary hypertension as a risk factor for death in patients with sickle cell disease. $N$ Engl J Med. 2004;350(9):886-95.

58. Anthi A, Machado RF, Jison ML, Taveira-Dasilva AM, Rubin LJ, Hunter L, et al. Hemodynamic and functional assessment of patients with sickle cell disease and pulmonary hypertension. Am J Respir Crit Care Med. 2007;175(12):1272-9.

59. Wallace RB. Screening for early and asymptomatic conditions. In: Wallace RB, editor. Public Health and Preventive Medicine. 14th ed. Norwalk: Appleton \& Lange; 1998. p. 907-8.

60. McLaughlin VV, Archer SL, Badesch DB, Barst RJ, Farber HW, Lindner JR, et al. ACCF/AHA 2009 expert consensus document on pulmonary hypertension a report of the American College of Cardiology Foundation Task Force on Expert Consensus Documents and the American Heart Association developed in collaboration with the American College of Chest Physicians; American Thoracic Society, Inc.; and the Pulmonary Hypertension Association. J Am Coll Cardiol. 2009;53(17):1573-619.

61. Castro O, Hoque M, Brown BD. Pulmonary hypertension in sickle cell disease: cardiac catheterization results and survival. Blood. 2003;101(4):1257-61.

62. Sylvester KP, Desai SR, Wells AU, Hansell DM, Awogbade M, Thein SL, et al. Computed tomography and pulmonary function abnormalities in sickle cell disease. Eur Respir J. 2006;28(4):832-8.

63. Jaja SI, Opesanwo O, Mojiminiyi FB, Kehinde MO. Lung function, haemoglobin and irreversibly sickled cells in sickle cell patients. West Afr J Med. 2000;19(3):225-9.

64. Holloman KL, Johnson CS, Haywood LJ. Electrocardiogram analysis in adult patients with sickle cell disease. J Natl Med Assoc. 1987;79(8):809-14.

65. Behera SK, Swain UK, Panda CP, Samal GC, Mohapatra SS. Cardiac state in sickle cell anaemia. Indian Pediatr. 1979;16(10):897-901.

66. Fontaine JM, Ofili EO, Adenaike MB, VanDecker W, Haywood LJ. Clinical assessment of the risk for sudden cardiac death in patients with sickle cell anemia. J Natl Med Assoc. 2008;100(4):360-8.

67. Barrett O, Jr., Saunders DE, Jr., McFarland DE, Humphries JO. Myocardial infarction in sickle cell anemia. Am J Hematol. 1984;16(2):139-47.

68. Alpert BS, Gilman PA, Strong WB, Ellison MF, Miller MD, McFarlane J, et al. Hemodynamic and ECG responses to exercise in children with sickle cell anemia. Am J Dis Child. 1981;135(4):362-6. 
69. Goldberg D, Tanel R, Kaltman J, Coffey K, Varela C, Smith-Whitley K. A prolonged QTC is not associated with increased mortality in sickle cell disease. American Society of Pediatric Hematology/Oncology 23rd Annual Meeting: Abstracts. Pediatr Blood Cancer. 2010;54(6):835-6.

70. Chobanian AV, Bakris GL, Black HR, Cushman WC, Green LA, Izzo JL Jr, et al. Seventh report of the Joint National Committee on Prevention, Detection, Evaluation, and Treatment of High Blood Pressure. Hypertension. 2003;42(6):1206-52.

71. Expert Panel on Integrated Guidelines for Cardiovascular Health and Risk Reduction in Children and Adolescents; National Heart, Lung, and Blood Institute. Expert panel on integrated guidelines for cardiovascular health and risk reduction in children and adolescents: summary report. Pediatrics. 2011;128 Suppl 5:S213-56.

72. Pegelow CH, Colangelo L, Steinberg M, Wright EC, Smith J, Phillips G, et al. Natural history of blood pressure in sickle cell disease: risks for stroke and death associated with relative hypertension in sickle cell anemia. Am J Med. 1997;102(2):171-7.

73. Rodgers GP, Walker EC, Podgor MJ. Is "relative" hypertension a risk factor for vaso-occlusive complications in sickle cell disease? Am J Med Sci. 1993;305(3):150-6.

74. DeBaun MR, Armstrong FD, McKinstry RC, Ware RE, Vichinsky E, Kirkham FJ. Silent cerebral infarcts: a review on a prevalent and progressive cause of neurologic injury in sickle cell anemia. Blood. 2012;119(20):4587-96.

75. Foucan L, Bourhis V, Bangou J, Merault L, Etienne-Julan M, Salmi RL. A randomized trial of captopril for microalbuminuria in normotensive adults with sickle cell anemia. Am J Med. 1998;104(4):339-42.

76. Uzun B, Kekec Z, Gurkan E. Efficacy of tramadol vs meperidine in vasoocclusive sickle cell crisis. Am J Emerg Med. 2010;28(4):445-9.

77. Ohene-Frempong K, Weiner SJ, Sleeper LA, Miller ST, Embury S, Moohr JW, et al. Cerebrovascular accidents in sickle cell disease: rates and risk factors. Blood. 1998;91(1):28894.

78. Strouse JJ, Hulbert ML, DeBaun MR, Jordan LC, Casella JF. Primary hemorrhagic stroke in children with sickle cell disease is associated with recent transfusion and use of corticosteroids. Pediatrics. 2006;118(5):1916-24.

79. Rogovik AL, Li Y, Kirby MA, Friedman JN, Goldman RD. Admission and length of stay due to painful vasoocclusive crisis in children. Am J Emerg Med. 2009;27(7):797-801.

80. Abo-Zenah H, Moharram M, El Nahas AM. Cardiorenal risk prevalence in sickle cell hemoglobinopathy. Nephron Clin Pract. 2009;112(2):c98-c106.

81. Nebor D, Broquere C, Brudey K, Mougenel D, Tarer V, Connes P, et al. Alpha-thalassemia is associated with a decreased occurrence and a delayed age-at-onset of albuminuria in sickle cell anemia patients. Blood Cells Mol Dis. 2010;45(2):154-8. 
82. National High Blood Pressure Education Program Working Group on High Blood Pressure in Children and Adolescents. The fourth report on the diagnosis, evaluation, and treatment of high blood pressure in children and adolescents. Pediatrics. 2004;114(2 Suppl 4th Report):555-76.

83. Moriarty BJ, Acheson RW, Condon PI, Serjeant GR. Patterns of visual loss in untreated sickle cell retinopathy. Eye (Lond). 1988;2 (Pt 3):330-5.

84. Downes SM, Hambleton IR, Chuang EL, Lois N, Serjeant GR, Bird AC. Incidence and natural history of proliferative sickle cell retinopathy: observations from a cohort study.

Ophthalmology. 2005;112(11):1869-75.

85. Rosenberg JB, Hutcheson KA. Pediatric sickle cell retinopathy: correlation with clinical factors. $J$ AAPOS. 2011;15(1):49-53.

86. Farber MD, Jampol LM, Fox P, Moriarty BJ, Acheson RW, Rabb MF, et al. A randomized clinical trial of scatter photocoagulation of proliferative sickle cell retinopathy. Arch Ophthalmol. 1991;109(3):363-7.

87. Jacobson MS, Gagliano DA, Cohen SB, Rabb MF, Jampol LM, Farber MD, et al. A randomized clinical trial of feeder vessel photocoagulation of sickle cell retinopathy. A longterm follow-up. Ophthalmology. 1991;98(5):581-5.

88. Jampol LM, Condon P, Farber M, Rabb M, Ford S, Serjeant G. A randomized clinical trial of feeder vessel photocoagulation of proliferative sickle cell retinopathy. I. Preliminary results. Ophthalmology. 1983;90(5):540-5.

89. Gill HS, Lam WC. A screening strategy for the detection of sickle cell retinopathy in pediatric patients. Can J Ophthalmol. 2008;43(2):188-91.

90. Friberg TR, Young CM, Milner PF. Incidence of ocular abnormalities in patients with sickle hemoglobinopathies. Annals of ophthalmology. 1986;18(4):150-3.

91. Talbot JF, Bird AC, Maude GH, Acheson RW, Moriarty BJ, Serjeant GR. Sickle cell retinopathy in Jamaican children: further observations from a cohort study. Br J Ophthalmol. 1988;72(10):727-32.

92. Verduzco LA, Nathan DG. Sickle cell disease and stroke. Blood. 2009;114(25):5117-25.

93. Dowling MM, Quinn CT, Rogers ZR, Buchanan GR. Acute silent cerebral infarction in children with sickle cell anemia. Pediatr Blood Cancer. 2010;54(3):461-4.

94. Ware RE, Zimmerman SA, Schultz WH. Hydroxyurea as an alternative to blood transfusions for the prevention of recurrent stroke in children with sickle cell disease. Blood. 1999;94(9):3022-6.

95. Adams R, McKie V, Nichols F, Carl E, Zhang DL, McKie K, et al. The use of transcranial ultrasonography to predict stroke in sickle cell disease. N Engl J Med. 1992;326(9):605-10.

96. Adams RJ, McKie VC, Hsu L, Files B, Vichinsky E, Pegelow C, et al. Prevention of a first stroke by transfusions in children with sickle cell anemia and abnormal results on transcranial Doppler ultrasonography. N Engl J Med. 1998;339(1):5-11. 
97. Enninful-Eghan H, Moore RH, Ichord R, Smith-Whitley K, Kwiatkowski JL. Transcranial Doppler ultrasonography and prophylactic transfusion program is effective in preventing overt stroke in children with sickle cell disease. J Pediatr. 2010;157(3):479-84.

98. Adams RJ, Brambilla D; Optimizing Primary Stroke Prevention in Sickle Cell Anemia (STOP 2) Trial Investigators. Discontinuing prophylactic transfusions used to prevent stroke in sickle cell disease. N Engl J Med. 2005;353(26):2769-78.

99. Strouse JJ, Lanzkron S, Urrutia V. The epidemiology, evaluation and treatment of stroke in adults with sickle cell disease. Expert Rev Hematol. 2011;4(6):597-606.

100. Silva GS, Vicari P, Figueiredo MS, Carrete H, Jr., Idagawa MH, Massaro AR. Brain magnetic resonance imaging abnormalities in adult patients with sickle cell disease: correlation with transcranial Doppler findings. Stroke. 2009;40(7):2408-12.

101. Adams RJ, Brambilla DJ, Granger S, Gallagher D, Vichinsky E, Abboud MR, et al. Stroke and conversion to high risk in children screened with transcranial Doppler ultrasound during the STOP study. Blood. 2004;103(10):3689-94.

102. Hankins JS, Fortner GL, McCarville MB, Smeltzer MP, Wang WC, Li CS, et al. The natural history of conditional transcranial Doppler flow velocities in children with sickle cell anaemia. Br J Haematol. 2008;142(1):94-9.

103. Makani J, Kirkham FJ, Komba A, Ajala-Agbo T, Otieno G, Fegan G, et al. Risk factors for high cerebral blood flow velocity and death in Kenyan children with Sickle Cell Anaemia: role of haemoglobin oxygen saturation and febrile illness. Br J Haematol. 2009;145(4):529-32.

104. Mazumdar M, Heeney MM, Sox CM, Lieu TA. Preventing stroke among children with sickle cell anemia: an analysis of strategies that involve transcranial Doppler testing and chronic transfusion. Pediatrics. 2007;120(4):e1107-16.

105. Boyd JH, Macklin EA, Strunk RC, DeBaun MR. Asthma is associated with acute chest syndrome and pain in children with sickle cell anemia. Blood. 2006;108(9):2923-7.

106. Boyd JH, Moinuddin A, Strunk RC, DeBaun MR. Asthma and acute chest in sickle-cell disease. Pediatr Pulmonol. 2004;38(3):229-32.

107. Knight-Madden JM, Forrester TS, Lewis NA, Greenough A. Asthma in children with sickle cell disease and its association with acute chest syndrome. Thorax. 2005;60(3):206-10.

108. Anim SO, Strunk RC, DeBaun MR. Asthma morbidity and treatment in children with sickle cell disease. Expert Rev Respir Med. 2011;5(5):635-45.

109. Cohen RT, Madadi A, Blinder MA, DeBaun MR, Strunk RC, Field JJ. Recurrent, severe wheezing is associated with morbidity and mortality in adults with sickle cell disease. Am J Hematol. 2011;86(9):756-61.

110. Kaleyias J, Mostofi N, Grant M, Coleman C, Luck L, Dampier C, et al. Severity of obstructive sleep apnea in children with sickle cell disease. J Pediatr Hematol Oncol. 2008;30(9):659-65. 
111. Field JJ, DeBaun MR, Yan Y, Strunk RC. Growth of lung function in children with sickle cell anemia. Pediatr Pulmonol. 2008;43(11):1061-6.

112. MacLean JE, Atenafu E, Kirby-Allen M, MacLusky IB, Stephens D, Grasemann H, et al. Longitudinal decline in lung volume in a population of children with sickle cell disease. Am J Respir Crit Care Med. 2008;178(10):1055-9.

113. Klings ES, Wyszynski DF, Nolan VG, Steinberg MH. Abnormal pulmonary function in adults with sickle cell anemia. Am J Respir Crit Care Med. 2006;173(11):1264-9.

114. Sylvester KP, Patey RA, Kassim Z, Rafferty GF, Rees D, Thein SL, et al. Lung gas transfer in children with sickle cell anaemia. Respir Physiol Neurobiol. 2007;158(1):70-4.

115. Koumbourlis AC, Lee DJ, Lee A. Lung function and somatic growth in patients with hemoglobin SC sickle cell disease. Pediatr Pulmonol. 2008;43(2):175-8.

116. Johnson K, Posner SF, Biermann J, Cordero JF, Atrash HK, Parker CS, et al.

Recommendations to improve preconception health and health care--United States. A report of the CDC/ATSDR Preconception Care Work Group and the Select Panel on Preconception Care. MMWR Recomm Rep. 2006;55(RR-6):1-23.

117. Barfield WD, Barradas DT, Manning SE, Kotelchuck M, Shapiro-Mendoza CK. Sickle cell disease and pregnancy outcomes: women of African descent. Am J Prev Med. 2010;38(4 Suppl):S542-9.

118. Villers MS, Jamison MG, De Castro LM, James AH. Morbidity associated with sickle cell disease in pregnancy. Am J Obstet Gynecol. 2008;199(2):125 e1-5.

119. American College of Obstetricians and Gynecologists Committee on Obstetrics. ACOG Practice Bulletin No. 78: hemoglobinopathies in pregnancy. Obstet Gynecol. 2007;109(1):22937.

120. Ngo C, Kayem G, Habibi A, Benachi A, Goffinet F, Galacteros F, et al. Pregnancy in sickle cell disease: maternal and fetal outcomes in a population receiving prophylactic partial exchange transfusions. Eur J Obstet Gynecol Reprod Biol. 2010;152(2):138-42.

121. Yu CK, Stasiowska E, Stephens A, Awogbade M, Davies A. Outcome of pregnancy in sickle cell disease patients attending a combined obstetric and haematology clinic. J Obstet Gynaecol. 2009;29(6):512-6.

122. Al Jama FE, Gasem T, Burshaid S, Rahman J, Al Suleiman SA, Rahman MS. Pregnancy outcome in patients with homozygous sickle cell disease in a university hospital, Eastern Saudi Arabia. Arch Gynecol Obstet. 2009;280(5):793-7.

123. Bonnin M, Mercier FJ, Sitbon O, Roger-Christoph S, Jais X, Humbert M, et al. Severe pulmonary hypertension during pregnancy: mode of delivery and anesthetic management of 15 consecutive cases. Anesthesiology. 2005;102(6):1133-7; discussion 5A-6A.

124. Weiss BM, Zemp L, Seifert B, Hess OM. Outcome of pulmonary vascular disease in pregnancy: a systematic overview from 1978 through 1996. J Am Coll Cardiol. 1998;31(7):1650-7. 
125. Yentis SM, Steer PJ, Plaat F. Eisenmenger's syndrome in pregnancy: maternal and fetal mortality in the 1990s. Br J Obstet Gynaecol. 1998;105(8):921-2.

126. Kiely DG, Condliffe R, Webster V, Mills GH, Wrench I, Gandhi SV, et al. Improved survival in pregnancy and pulmonary hypertension using a multiprofessional approach. Br J Obstet Gynaecol. 2010;117(5):565-74.

127. Legardy JK, Curtis KM. Progestogen-only contraceptive use among women with sickle cell anemia: a systematic review. Contraception. 2006;73(2):195-204.

128. Manchikanti A, Grimes DA, Lopez LM, Schulz KF. Steroid hormones for contraception in women with sickle cell disease. Cochrane Database Syst Rev. 2007;Apr 18(2):CD006261.

129. World Health Organization. Medical eligibility for contraceptive use. 4th edition. Geneva: World Health Organization Press, 2009.

130. Adadevoh BK, Isaacs WA. The effect of megestrol acetate on sickling. Am J Med Sci. 1973;265(5):367-70.

131. Barbosa IC, Ladipo OA, Nascimento ML, Athayde C, Hirsch C, Lopes R, et al. Carbohydrate metabolism in sickle cell patients using a subdermal implant containing nomegestrol acetate (Uniplant). Contraception. 2001;63(5):263-5.

132. de Abood M, de Castillo Z, Guerrero F, Espino M, Austin KL. Effect of Depo-Provera or Microgynon on the painful crises of sickle cell anemia patients. Contraception. 1997;56(5):313-6.

133. De Ceulaer K, Gruber C, Hayes R, Serjeant GR. Medroxyprogesterone acetate and homozygous sickle-cell disease. Lancet. 1982;2(8292):229-31.

134. Howard RJ, Lillis C, Tuck SM. Contraceptives, counselling, and pregnancy in women with sickle cell disease. BMJ. 1993;306(6894):1735-7.

135. Ladipo OA, Falusi AG, Feldblum PJ, Osotimehin BO, Otolorin EO, Ojengbede OA. Norplant use by women with sickle cell disease. Int J Gynaecol Obstet. 1993;41(1):85-7.

136. Nascimento M, Ladipo OA, Coutinho EM. Nomegestrol acetate contraceptive implant use by women with sickle cell disease. Clin Pharmacol Ther. 1998;64(4):433-8.

137. Yoong WC, Tuck SM, Yardumian A. Red cell deformability in oral contraceptive pill users with sickle cell anaemia. Br J Haematol. 1999;104(4):868-70.

138. Executive Office of Health and Human Services, Massachusetts Department of Public Health Immunization Program. Summary of ACIP Recommended Groups for Vaccination [Internet]. [cited 2013 Feb 13]. Available from:

http://www.mass.gov/eohhs/docs/dph/cdc/immunization/acip-summary-recommendedgroups.pdf. 
139. Bollman A. The History of Vaccines Blog [Internet]. Philadelphia: The College of Physicians of Philadelphia. 2012. [cited 2012 Oct 26]. Available from:

http://www.historyofvaccines.org/content/blog/acip-makes-new-tdap-and-meningococcalvaccine-recommendations.

140. Use of 13-valent pneumococcal conjugate vaccine and 23-valent pneumococcal polysaccharide vaccine for adults with immunocompromising conditions: recommendations of the Advisory Committee on Immunization Practices (ACIP). MMWR Morb Mortal Wkly Rpt. 2012;61(40):816-9.

141. Kochanek KD, Xu J, Murphy SL, Minino AM, Kung H. Deaths: preliminary data for 2009. National Vital Statistics Report. 2011.

142. Platt OS, Thorington BD, Brambilla DJ, Milner PF, Rosse WF, Vichinsky E, et al. Pain in sickle cell disease. Rates and risk factors. N Engl J Med. 1991;325(1):11-6.

143. Ballas SK, Lusardi M. Hospital readmission for adult acute sickle cell painful episodes: frequency, etiology, and prognostic significance. Am J Hematol. 2005;79(1):17-25.

144. Jacob E, Beyer JE, Miaskowski C, Savedra M, Treadwell M, Styles L. Are there phases to the vaso-occlusive painful episode in sickle cell disease? J Pain Symptom Manage. 2005;29(4):392-400.

145. Smith WR, Penberthy LT, Bovbjerg VE, McClish DK, Roberts JD, Dahman B, et al. Daily assessment of pain in adults with sickle cell disease. Ann Intern Med. 2008;148(2):94-101.

146. Houston-Yu P, Rana SR, Beyer B, Castro O. Frequent and prolonged hospitalizations: a risk factor for early mortality in sickle cell disease patients. Am J Hematol. 2003;72(3):201-3.

147. Ballas SK. Pain management of sickle cell disease. Hematol Oncol Clin North Am. 2005;19(5):785-802, v.

148. Ballas SK. The sickle cell painful crisis in adults: phases and objective signs. Hemoglobin. 1995;19(6):323-33.

149. Gilboy N, Tanabe P, Travers DA, Rosenau AM. Emergency severity index (ESI): a triage tool for emergency department care, version 4. Implementation handbook 2012 edition. Rockville (MD): Agency for Healthcare Research and Quality (US), 2011 Nov. (AHRQ Publication No. 12-0014). Report No.

150. Gonzalez ER, Ornato JP, Ware D, Bull D, Evens RP. Comparison of intramuscular analgesic activity of butorphanol and morphine in patients with sickle cell disease. Ann Emerg Med. 1988;17(8):788-91.

151. Jacobson SJ, Kopecky EA, Joshi P, Babul N. Randomised trial of oral morphine for painful episodes of sickle-cell disease in children. Lancet. 1997;350(9088):1358-61.

152. Wright SW, Norris RL, Mitchell TR. Ketorolac for sickle cell vaso-occlusive crisis pain in the emergency department: lack of a narcotic-sparing effect. Ann Emerg Med. 1992;21(8):925-8. 
153. Brookoff D, Polomano R. Treating sickle cell pain like cancer pain. Ann Intern Med. 1992;116(5):364-8.

154. Chou R, Ballantyne JC, Fanciullo GJ, Fine PG, Miaskowski C. Research gaps on use of opioids for chronic noncancer pain: findings from a review of the evidence for an American Pain Society and American Academy of Pain Medicine clinical practice guideline. J Pain. 2009;10(2):147-59.

155. Udezue E, Herrera E. Pain management in adult acute sickle cell pain crisis: a viewpoint. West Afr J Med. 2007;26(3):179-82.

156. Gonzalez ER, Bahal N, Hansen LA, Ware D, Bull DS, Ornato JP, et al. Intermittent injection vs patient-controlled analgesia for sickle cell crisis pain. Comparison in patients in the emergency department. Arch Intern Med. 1991;151(7):1373-8.

157. Hardwick WE, Jr., Givens TG, Monroe KW, King WD, Lawley D. Effect of ketorolac in pediatric sickle cell vaso-occlusive pain crisis. Pediatr Emerg Care. 1999;15(3):179-82.

158. Perlin E, Finke H, Castro O, Adir J, Pittman J, Ki Moon B. Infusional/patient-controlled analgesia in sickle-cell vaso-occlusive crises. Pain Clinic. 1993;6(2):113-19.

159. van Beers EJ, van Tuijn CF, Nieuwkerk PT, Friederich PW, Vranken JH, Biemond BJ. Patientcontrolled analgesia versus continuous infusion of morphine during vaso-occlusive crisis in sickle cell disease, a randomized controlled trial. Am J Hematol. 2007;82(11):955-60.

160. Cole TB, Sprinkle RH, Smith SJ, Buchanan GR. Intravenous narcotic therapy for children with severe sickle cell pain crisis. Am J Dis Child. 1986;140(12):1255-9.

161. Holbrook CT. Patient-controlled analgesia pain management for children with sickle cell disease. J Assoc Acad Minor Phys. 1990;1(3):93-6.

162. Booth C, Inusa B, Obaro SK. Infection in sickle cell disease: a review. Int J Infect Dis. 2010;14(1):e2-e12.

163. Hassell KL, Eckman JR, Lane PA. Acute multiorgan failure syndrome: a potentially catastrophic complication of severe sickle cell pain episodes. Am J Med. 1994;96(2):155-62.

164. Pham PT, Pham PC, Wilkinson AH, Lew SQ. Renal abnormalities in sickle cell disease. Kidney Int. 2000;57(1):1-8.

165. Chehal A, Taher A, Shamseddine A. Sicklemia with multi-organ failure syndrome and thrombotic thrombocytopenic purpura. Hemoglobin. 2002;26(4):345-51.

166. Kelly CJ, Singer I. Acute renal failure in sickle-cell disease. Am J Kidney Dis. 1986;8(3):14650 .

167. Davenport A, Buscombe J. Sickle cell kidney. J Nephrol. 2008;21(2):253-5.

168. Couillard S, Benkerrou M, Girot R, Brousse V, Ferster A, Bader-Meunier B. Steroid treatment in children with sickle-cell disease. Haematologica. 2007;92(3):425-6. 
169. Little JA, McGowan VR, Kato GJ, Partovi KS, Feld JJ, Maric I, et al. Combination erythropoietin-hydroxyurea therapy in sickle cell disease: experience from the National Institutes of Health and a literature review. Haematologica. 2006;91(8):1076-83.

170. Ojo AO, Govaerts TC, Schmouder RL, Leichtman AB, Leavey SF, Wolfe RA, et al. Renal transplantation in end-stage sickle cell nephropathy. Transplantation. 1999;67(2):291-5.

171. Warady BA, Sullivan EK. Renal transplantation in children with sickle cell disease: a report of the North American Pediatric Renal Transplant Cooperative Study (NAPRTCS). Pediatr Transplant. 1998;2(2):130-3.

172. Adeyoju AB, Olujohungbe AB, Morris J, Yardumian A, Bareford D, Akenova A, et al. Priapism in sickle-cell disease; incidence, risk factors and complications - an international multicentre study. BJU Int. 2002;90(9):898-902.

173. Olujohungbe AB, Adeyoju A, Yardumian A, Akinyanju O, Morris J, Westerdale N, et al. A prospective diary study of stuttering priapism in adolescents and young men with sickle cell anemia: report of an international randomized control trial-The Priapism in Sickle Cell Study. J Androl. 2011;32(4):375-82.

174. Serjeant GR, de Ceulaer K, Maude GH. Stilboestrol and stuttering priapism in homozygous sickle-cell disease. Lancet. 1985;2(8467):1274-6.

175. Rachid-Filho D, Cavalcanti AG, Favorito LA, Costa WS, Sampaio FJ. Treatment of recurrent priapism in sickle cell anemia with finasteride: a new approach. Urology. 2009;74(5):1054-7.

176. Costabile RA. Successful treatment of stutter priapism with an antiandrogen. Tech Urol. 1998;4(3):167-8.

177. Dahm P, Rao DS, Donatucci CF. Antiandrogens in the treatment of priapism. Urology. 2002;59(1):138.

178. Hoffman S, Kaynan AM, Melman A. Priapism of ambiguous classification in a sickle cell patient. Int J Impot Res. 2000;12(1):59-63.

179. Levine LA, Guss SP. Gonadotropin-releasing hormone analogues in the treatment of sickle cell anemia-associated priapism. J Urol. 1993;150(2 Pt 1):475-7.

180. Adeyokunnu AA, Lawani JO, Nkposong EO. Priapism complicating sickle cell disease in Nigerian children. Ann Trop Paediatr. 1981;1(3):143-7.

181. Enwerem EO, Endeley EM, Holcombe C, Patel RV. Priapism in children with sickle-cell disease. Indian Pediatr. 1992;29(8):1048-9.

182. McHardy P, McDonnell C, Lorenzo AJ, Salle JL, Campbell FA. Management of priapism in a child with sickle cell anemia; successful outcome using epidural analgesia. Can J Anaesth. 2007;54(8):642-5.

183. Colombani JF, Peluchon P, Elana G, Delatre P. Priapism in a sickle cell prepuberal child. Eur J Pediatr Surg. 2000;10(1):68-71. 
184. McCarthy LJ, Vattuone J, Weidner J, Skipworth E, Fernandez C, Jackson L, et al. Do automated red cell exchanges relieve priapism in patients with sickle cell anemia? Ther Apher. 2000;4(3):256-8.

185. Al Jam'a AH, Al Dabbous IA. Hydroxyurea in the treatment of sickle cell associated priapism. J Urol. 1998;159(5):1642.

186. el Mauhoub M, el Bargathy S, Sabharwal HS, Aggarwal VP, el Warrad K. Priapism in sickle cell anaemia: a case report. Ann Trop Paediatr. 1991;11(4):371-2.

187. Fich A, Rachmilewitz EA. Priapism in a non-black with sickle cell anemia associated with alpha-thalassemia. Am J Hematol. 1981;10(3):313-5.

188. Hamre MR, Harmon EP, Kirkpatrick DV, Stern MJ, Humbert JR. Priapism as a complication of sickle cell disease. J Urol. 1991;145(1):1-5.

189. Kleinman S, Thompson-Breton R, Rifkind S, Goldfinger D. Exchange red blood cell pheresis in the management of complications of sickle cell anemia. Plasma Ther Transfus Technol. 1980;1(3):27-34.

190. Kinney TR, Harris MB, Russell MO, Duckett J, Schwartz E. Priapism in association with sickle hemoglobinopathies in children. J Pediatr. 1975;86(2):241-2.

191. Rifkind S, Waisman J, Thompson R, Goldfinger D. RBC exchange pheresis for priapism in sickle cell disease. JAMA. 1979;242(21):2317-8.

192. Slayton W, Kedar A, Schatz D. Testosterone induced priapism in two adolescents with sickle cell disease. J Pediatr Endocrinol Metab. 1995;8(3):199-203.

193. Walker EM Jr, Mitchum EN, Rous SN, Glassman AB, Cannon A, McInnes BK 3rd. Automated erythrocytopheresis for relief of priapism in sickle cell hemoglobinopathies. J Urol. 1983;130(5):912-6.

194. Rackoff WR, Ohene-Frempong K, Month S, Scott JP, Neahring B, Cohen AR. Neurologic events after partial exchange transfusion for priapism in sickle cell disease. J Pediatr. 1992;120(6):882-5.

195. Gradisek RE. Priapism in sickle cell disease. Ann Emerg Med. 1983;12(8):510-2.

196. Karayalcin G, Imran M, Rosner F. Priapism in sickle cell disease: report of five cases. Am J Med Sci. 1972;264(4):289-93.

197. Dunn EK, Miller ST, Macchia RJ, Glassberg KI, Gillette PN, Sarkar SD, et al. Penile scintigraphy for priapism in sickle cell disease. J Nucl Med. 1995;36(8):1404-7.

198. Muneer A, Garaffa G, Minhas S, Ralph DJ. The management of stuttering priapism within a specialist unit: a 25-year experience. Br J Med Surg Urol. 2009;2(1):11-6.

199. Mantadakis E, Ewalt DH, Cavender JD, Rogers ZR, Buchanan GR. Outpatient penile aspiration and epinephrine irrigation for young patients with sickle cell anemia and prolonged priapism. Blood. 2000;95(1):78-82. 
200. Davila HH, Parker J, Webster JC, Lockhart JL, Carrion RE. Subarachnoid hemorrhage as complication of phenylephrine injection for the treatment of ischemic priapism in a sickle cell disease patient. J Sex Med. 2008;5(4):1025-8.

201. Gbadoe AD, Atakouma Y, Kusiaku K, Assimadi JK. Management of sickle cell priapism with etilefrine. Arch Dis Child. 2001;85(1):52-3.

202. Liu J, Al-Hothari MA, Mahboob FA. Non surgical treatment of recurrent or stuttering priapism in sickle cell children. Saudi Med J. 2003;24(10):1143-5.

203. Virag R, Bachir D, Lee K, Galacteros F. Preventive treatment of priapism in sickle cell disease with oral and self-administered intracavernous injection of etilefrine. Urology. 1996;47(5):77781 ; discussion 81 .

204. Burnett AL, Bivalacqua TJ, Champion HC, Musicki B. Long-term oral phosphodiesterase 5 inhibitor therapy alleviates recurrent priapism. Urology. 2006;67(5):1043-8.

205. Adetayo FO. Outcome of management of acute prolonged priapism in patients with homozygous sickle cell disease. West Afr J Med. 2009;28(4):234-9.

206. El Morsi AR. A report on five cases of priapism. J Egypt Med Assoc. 1973;56(3):277-9.

207. Koirala S, Penagaluri P, Smith C, Lippmann S. Priapism and risperidone. Southern Med J. 2009;102(12):1266-8.

208. Noe HN, Wilimas J, Jerkins GR. Surgical management of priapism in children with sickle cell anemia. J Urol. 1981;126(6):770-1.

209. Winter CC. Priapism treated by modification of creation of fistulas between glans penis and corpora cavernosa. Trans Am Assoc Genitourin Surg. 1978;70:88-9.

210. Green TW, Conley CL, Berthrong M. [The liver in sickle cell anemia]. Bull Johns Hopkins Hosp. 1953;92(2):99-127.

211. Al-Mulhim AS, Al-Mulhim FM, Al-Suwaiygh AA. The role of laparoscopic cholecystectomy in the management of acute cholecystitis in patients with sickle cell disease. Am J Surg. 2002;183(6):668-72.

212. Sarnaik S, Slovis TL, Corbett DP, Emami A, Whitten CF. Incidence of cholelithiasis in sickle cell anemia using the ultrasonic gray-scale technique. J Pediatr. 1980;96(6):1005-8.

213. Curro G, Iapichino G, Lorenzini C, Palmeri R, Cucinotta E. Laparoscopic cholecystectomy in children with chronic hemolytic anemia. Is the outcome related to the timing of the procedure? Surg Endosc. 2006;20(2):252-5.

214. Vichinsky EP, Lubin BH. Sickle cell anemia and related hemoglobinopathies. Pediatr Clin North Am. 1980;27(2):429-47.

215. Ballas SK, Lewis CN, Noone AM, Krasnow SH, Kamarulzaman E, Burka ER. Clinical, hematological, and biochemical features of Hb SC disease. Am J Hematol. 1982;13(1):37-51. 
216. Meshikhes AW, Al-Abkari HA, Al-Faraj AA, Al-Dhurais SA, Al-Saif O. The safety of laparoscopic cholecystectomy in sickle cell disease: An update. Ann Saudi Med. 1998;18(1):124.

217. Spigelman A, Warden MJ. Surgery in patients with sickle cell disease. Arch Surg. 1972;104(6):761-4.

218. McCall IW, Desai P, Serjeant BE, Serjeant GR. Cholelithiasis in Jamaican patients with homozygous sickle cell disease. Am J Hematol. 1977;3:15-21.

219. Lee SP, Maher K, Nicholls JF. Origin and fate of biliary sludge. Gastroenterology. 1988;94(1):170-6.

220. Cameron JL, Maddrey WC, Zuidema GD. Biliary tract disease in sickle cell anemia: surgical considerations. Ann Surg. 1971;174(4):702-10.

221. Lee SP, Nicholls JF, Park HZ. Biliary sludge as a cause of acute pancreatitis. $N$ Engl J Med. 1992;326(9):589-93.

222. Ahn H, Li CS, Wang W. Sickle cell hepatopathy: clinical presentation, treatment, and outcome in pediatric and adult patients. Pediatr Blood Cancer. 2005;45(2):184-90.

223. Hatton CS, Bunch C, Weatherall DJ. Hepatic sequestration in sickle cell anaemia. Br Med J (Clin Res Ed). 1985;290(6470):744-5.

224. Al-Salem AH, Qaisruddin S. The significance of biliary sludge in children with sickle cell disease. Pediatr Surg Int. 1998;13(1):14-6.

225. Walker TM, Serjeant GR. Biliary sludge in sickle cell disease. J Pediatr. 1996;129(3):443-5.

226. Irizarry K, Rossbach HC, Ignacio JR, Winesett MP, Kaiser GC, Kumar M, et al. Sickle cell intrahepatic cholestasis with cholelithiasis. Pediatr Hematol Oncol. 2006;23(2):95-102.

227. Stephan JL, Merpit-Gonon E, Richard O, Raynaud-Ravni C, Freycon F. Fulminant liver failure in a 12-year-old girl with sickle cell anaemia: favourable outcome after exchange transfusions. Eur J Pediatr. 1995;154(6):469-71.

228. Shao SH, Orringer EP. Sickle cell intrahepatic cholestasis: approach to a difficult problem. $\mathrm{Am}$ J Gastroenterol. 1995;90(11):2048-50.

229. Baichi MM, Arifuddin RM, Mantry PS, Bozorgzadeh A, Ryan C. Liver transplantation in sickle cell anemia: a case of acute sickle cell intrahepatic cholestasis and a case of sclerosing cholangitis. Transplantation. 2005;80(11):1630-2.

230. Costa DB, Miksad RA, Buff MS, Wang Y, Dezube BJ. Case of fatal sickle cell intrahepatic cholestasis despite use of exchange transfusion in an African-American patient. J Natl Med Assoc. 2006;98(7):1183-7.

231. Davies SC, Brozovic M. The presentation, management and prophylaxis of sickle cell disease. Blood Rev. 1989;3(1):29-44. 
232. Hernandez RJ, Sarnaik SA, Lande I, Aisen AM, Glazer GM, Chenevert T, et al. MR evaluation of liver iron overload. J Comput Assist Tomogr. 1988;12(1):91-4.

233. Walker TM, Hambleton IR, Serjeant GR. Gallstones in sickle cell disease: observations from The Jamaican Cohort study. J Pediatr. 2000;136(1):80-5.

234. Leandros E, Kymionis GD, Konstadoulakis MM, Albanopoulos K, Dimitrakakis K, Gomatos I, et al. Laparoscopic or open cholecystectomy in patients with sickle cell disease: which approach is superior? Eur J Surg. 2000;166(11):859-61.

235. Tagge EP, Othersen HB, Jr., Jackson SM, Smith CD, Gayoso AJ, Abboud MR, et al. Impact of laparoscopic cholecystectomy on the management of cholelithiasis in children with sickle cell disease. J Pediatr Surg. 1994;29(2):209-12; discussion 12-3.

236. Jawad AJ, Kurban K, el-Bakry A, al-Rabeeah A, Seraj M, Ammar A. Laparoscopic cholecystectomy for cholelithiasis during infancy and childhood: cost analysis and review of current indications. World J Surg. 1998;22(1):69-73; discussion 4.

237. Smith-Whitley K, Zhao H, Hodinka RL, Kwiatkowski J, Cecil R, Cecil T, et al. Epidemiology of human parvovirus B19 in children with sickle cell disease. Blood. 2004;103(2):422-7.

238. Serjeant GR, Topley JM, Mason K, Serjeant BE, Pattison JR, Jones SE, et al. Outbreak of aplastic crises in sickle cell anaemia associated with parvovirus-like agent. Lancet. 1981;2(8247):595-7.

239. Topley JM, Rogers DW, Stevens MC, Serjeant GR. Acute splenic sequestration and hypersplenism in the first five years in homozygous sickle cell disease. Arch Dis Child. 1981;56(10):765-9.

240. Aquino VM, Norvell JM, Buchanan GR. Acute splenic complications in children with sickle cell-hemoglobin C disease. J Pediatr. 1997;130(6):961-5.

241. Wright JG, Hambleton IR, Thomas PW, Duncan ND, Venugopal S, Serjeant GR. Postsplenectomy course in homozygous sickle cell disease. J Pediatr. 1999;134(3):304-9.

242. Kinney TR, Ware RE, Schultz WH, Filston HC. Long-term management of splenic sequestration in children with sickle cell disease. J Pediatr. 1990;117(2 Pt 1):194-9.

243. Buchanan GR, McKie V, Jackson EA, Vedro DA, Hamner S, Holtkamp CA. Splenic phagocytic function in children with sickle cell anemia receiving long-term hypertransfusion therapy. J Pediatr. 1989;115(4):568-72.

244. Rezende PV, Viana MB, Murao M, Chaves AC, Ribeiro AC. Acute splenic sequestration in a cohort of children with sickle cell anemia. J Pediatr. 2009;85(2):163-9.

245. Subbannan K, Ustun C, Natarajan K, Clair B, Daitch L, Fields S, et al. Acute splenic complications and implications of splenectomy in hemoglobin SC disease. Eur J Haematol. 2009;83(3):258-60.

246. Grover R, Wethers DL. Management of acute splenic sequestration crisis in sickle cell disease. J Assoc Acad Minor Phys. 1990;1(3):67-70. 
247. Santos A, Pinheiro V, Anjos C, Brandalise S, Fahel F, Lima M, et al. Scintigraphic follow-up of the effects of therapy with hydroxyurea on splenic function in patients with sickle cell disease. Eur J Nucl Med Mol Imaging. 2002;29(4):536-41.

248. Rao S, Gooden S. Splenic sequestration in sickle cell disease: role of transfusion therapy. Am $J$ Pediatr Hematol Oncol. 1985;7(3):298-301.

249. Salamah MM, Mallouh AA, Hamdan JA. Acute splenic sequestration crises in Saudi children with sickle cell disease. Ann Trop Paediatr. 1989;9(2):115-7.

250. Charache S, Scott JC, Charache P. "Acute chest syndrome" in adults with sickle cell anemia. Microbiology, treatment, and prevention. Arch Intern Med. 1979;139(1):67-9.

251. Vichinsky EP, Neumayr LD, Earles AN, Williams R, Lennette ET, Dean D, et al. Causes and outcomes of the acute chest syndrome in sickle cell disease. National Acute Chest Syndrome Study Group. $N$ Engl J Med. 2000;342(25):1855-65. [Erratum appears in N Engl J Med. 2000 Sep 14;343(11):824].

252. Vichinsky EP, Styles LA, Colangelo LH, Wright EC, Castro O, Nickerson B. Acute chest syndrome in sickle cell disease: clinical presentation and course. Cooperative Study of Sickle Cell Disease. Blood. 1997;89(5):1787-92.

253. Miller ST. How I treat acute chest syndrome in children with sickle cell disease. Blood. 2011;117(20):5297-305.

254. Bernini JC, Rogers ZR, Sandler ES, Reisch JS, Quinn CT, Buchanan GR. Beneficial effect of intravenous dexamethasone in children with mild to moderately severe acute chest syndrome complicating sickle cell disease. Blood. 1998;92(9):3082-9.

255. Sobota A, Graham DA, Heeney MM, Neufeld EJ. Corticosteroids for acute chest syndrome in children with sickle cell disease: variation in use and association with length of stay and readmission. Am J Hematol. 2010;85(1):24-8.

256. Buchanan ID, Woodward M, Reed GW. Opioid selection during sickle cell pain crisis and its impact on the development of acute chest syndrome. Pediatr Blood Cancer. 2005;45(5):71624.

257. King D, Conway EE Jr. Acute Chest Syndrome (ACS) in pediatric patients admitted to a Pediatric Critical Care Unit (PCCU) [Abstract]. Chest. 1996;110(Suppl. 4):1485.

258. Liem RI, O'Gorman MR, Brown DL. Effect of red cell exchange transfusion on plasma levels of inflammatory mediators in sickle cell patients with acute chest syndrome. Am J Hematol. 2004;76(1):19-25.

259. Miller ST, Macklin EA, Pegelow CH, Kinney TR, Sleeper LA, Bello JA, et al. Silent infarction as a risk factor for overt stroke in children with sickle cell anemia: a report from the Cooperative Study of Sickle Cell Disease. J Pediatr. 2001;139(3):385-90.

260. Cohen AR, Martin MB, Silber JH, Kim HC, Ohene-Frempong K, Schwartz E. A modified transfusion program for prevention of stroke in sickle cell disease. Blood. 1992;79(7):1657-61. 
261. Russell MO, Goldberg HI, Hodson A, Kim HC, Halus J, Reivich M, et al. Effect of transfusion therapy on arteriographic abnormalities and on recurrence of stroke in sickle cell disease.

Blood. 1984;63(1):162-9.

262. Wang WC, Kovnar EH, Tonkin IL, Mulhern RK, Langston JW, Day SW, et al. High risk of recurrent stroke after discontinuance of five to twelve years of transfusion therapy in patients with sickle cell disease. J Pediatr. 1991;118(3):377-82.

263. Wilimas J, Goff JR, Anderson HR, Jr., Langston JW, Thompson E. Efficacy of transfusion therapy for one to two years in patients with sickle cell disease and cerebrovascular accidents. $J$ Pediatr. 1980;96(2):205-8.

264. Scothorn DJ, Price C, Schwartz D, Terrill C, Buchanan GR, Shurney W, et al. Risk of recurrent stroke in children with sickle cell disease receiving blood transfusion therapy for at least five years after initial stroke. J Pediatr. 2002;140(3):348-54.

265. Pegelow CH, Adams RJ, McKie V, Abboud M, Berman B, Miller ST, et al. Risk of recurrent stroke in patients with sickle cell disease treated with erythrocyte transfusions. J Pediatr. 1995;126(6):896-9.

266. Hulbert ML, Scothorn DJ, Panepinto JA, Scott JP, Buchanan GR, Sarnaik S, et al. Exchange blood transfusion compared with simple transfusion for first overt stroke is associated with a lower risk of subsequent stroke: a retrospective cohort study of 137 children with sickle cell anemia. J Pediatr. 2006;149(5):710-2.

267. Ware RE, Schultz WH, Yovetich N, Mortier NA, Alvarez O, Hilliard L, et al. Stroke With Transfusions Changing to Hydroxyurea (SWiTCH): a phase III randomized clinical trial for treatment of children with sickle cell anemia, stroke, and iron overload. Pediatr Blood Cancer. 2011;57(6):1011-7.

268. Ware RE, Helms RW. Stroke With Transfusions Changing to Hydroxyurea (SWiTCH). Blood. 2012;119(17):3925-32.

269. Sumoza A, de Bisotti R, Sumoza D, Fairbanks V. Hydroxyurea (HU) for prevention of recurrent stroke in sickle cell anemia (SCA). Am J Hematol. 2002;71(3):161-5.

270. Ware RE, Zimmerman SA, Sylvestre PB, Mortier NA, Davis JS, Treem WR, et al. Prevention of secondary stroke and resolution of transfusional iron overload in children with sickle cell anemia using hydroxyurea and phlebotomy. J Pediatr. 2004;145(3):346-52.

271. Goldberg MF. The diagnosis and treatment of secondary glaucoma after hyphema in sickle cell patients. Am J Ophthalmol. 1979;87(1):43-9.

272. Lai JC, Fekrat S, Barron Y, Goldberg MF. Traumatic hyphema in children: risk factors for complications. Arch Ophthalmol. 2001;119(1):64-70.

273. Walton W, Von Hagen S, Grigorian R, Zarbin M. Management of traumatic hyphema. Surv Ophthalmol. 2002;47(4):297-334.

274. Gharaibeh A, Savage HI, Scherer RW, Goldberg MF, Lindsley K. Medical interventions for traumatic hyphema. Cochrane Database Syst Rev. 2011(1):CD005431. 
275. Deutsch TA, Weinreb RN, Goldberg MF. Indications for surgical management of hyphema in patients with sickle cell trait. Arch Ophthalmol. 1984;102(4):566-9.

276. Liem RI, Calamaras DM, Chhabra MS, Files B, Minniti CP, Thompson AA. Sudden-onset blindness in sickle cell disease due to retinal artery occlusion. Pediatr Blood Cancer. 2008;50(3):624-7.

277. Yanoff M, Duker JS, editors. Ophthalmology. St. Louis, MO: Mosby, Inc.; 2004.

278. Goodwin PL, Vaphiades MS, Johnson AP, Stroud CE. Bilateral central retinal artery occlusion associated with moyamoya syndrome in a sickle cell disease patient. Neuroophthamology. 2008;32(1):21-6.

279. Al-Abdulla NA, Haddock TA, Kerrison JB, Goldberg MF. Sickle cell disease presenting with extensive peri-macular arteriolar occlusions in a nine-year-old boy. Am J Ophthalmol. 2001;131(2):275-6.

280. Fine LC, Petrovic V, Irvine AR, Bhisitkul RB. Spontaneous central retinal artery occlusion in hemoglobin sickle cell disease. Am J Ophthalmol. 2000;129(5):680-1.

281. Marcus RE, Bolger JP, Roderick PJ, Rowson NJ. Central retinal artery occlusion in homozygous sickle cell (SS) disease. Clin Lab Haematol. 1988;10(4):467-70.

282. Clarke WN, Vomiero G, Leonard BC. Bilateral simultaneous retinal arteriolar obstruction in a child with hemoglobin SS sickle cell disease. J AAPOS. 2001;5(2):126-8.

283. Curran EL, Fleming JC, Rice K, Wang WC. Orbital compression syndrome in sickle cell disease. Ophthalmology. 1997;104(10):1610-5.

284. Wolff MH, Sty JR. Orbital infarction in sickle cell disease. Pediatr Radiol. 1985;15(1):50-2.

285. Sokol JA, Baron E, Lantos G, Kazim M. Orbital compression syndrome in sickle cell disease. Ophthal Plast Reconstr Surg. 2008;24(3):181-4.

286. Ballas SK. Sickle cell pain. Progress in pain research and management. Vol. 11. 11. Seattle, WA: IASP Press; 1998.

287. Ballas SK, Lieff S, Benjamin LJ, Dampier CD, Heeney MM, Hoppe C, et al. Definitions of the phenotypic manifestations of sickle cell disease. Am J Hematol. 2010;85(1):6-13.

288. Dampier C, Ely B, Brodecki D, O'Neal P. Characteristics of pain managed at home in children and adolescents with sickle cell disease by using diary self-reports. J Pain. 2002;3(6):461-70.

289. Ballas SK, Bauserman RL, McCarthy WF, Castro OL, Smith WR, Waclawiw MA; Investigators of the Multicenter Study of Hydroxyurea in Sickle Cell Anemia. Hydroxyurea and acute painful crises in sickle cell anemia: effects on hospital length of stay and opioid utilization during hospitalization, outpatient acute care contacts, and at home. J Pain Symptom Manage. 2010;40(6):870-82.

290. Konotey-Ahulu FI. Mental-nerve neuropathy: a complication of sickle-cell crisis. Lancet. 1972;2(7773):388. 
291. Kirson LE, Tomaro AJ. Mental nerve paresthesia secondary to sickle-cell crisis. Oral Surg Oral Med Oral Pathol. 1979;48(6):509-12.

292. Ballas SK, Reyes PF. Peripheral neuropathy in adults with sickle cell disease. Am J Pain Med. 1997;71:53-8.

293. Serjeant DR, Serjeant BE. Sickle cell disease. New York: Oxford University Press; 2001.

294. Alvim RC, Viana MB, Pires MA, Franklin HM, Paula MJ, Brito AC, et al. Inefficacy of piracetam in the prevention of painful crises in children and adolescents with sickle cell disease. Acta Haematol. 2005;113(4):228-33.

295. Ballas SK, Viscusi ER, Epstein KR. Management of acute chest wall sickle cell pain with nebulized morphine. Am J Hematol. 2004;76(2):190-1.

296. Myers CD, Robinson ME, Guthrie TH, Lamp SP, Lottenberg R. Adjunctive approaches for sickle cell phronic pain. Complement Health Pract Rev. 1999;5(3):203-12.

297. Ballas SK. Update on pain management in sickle cell disease. Hemoglobin. 2011;35(5-6):5209.

298. Hong YK, Chen C. Wiring and rewiring of the retinogeniculate synapse. Curr Opin Neurobiol. 2011;21(2):228-37.

299. Embury SH, Hebbel RP, Mohandas S, editors. Sickle cell disease. Basic principles and clinical picture. New York: Raven Press; 1994.

300. Odebiyi DO, Adigun OT, Kehinde MO. Effect of sodium salicylate iontophoresis in the management of hip pain in patients with sickle cell disease. Nig Q J Hosp Med. 2007;17(2):826.

301. Bodhise PB, Dejoie M, Brandon Z, Simpkins S, Ballas SK. Non-pharmacologic management of sickle cell pain. Hematology. 2004;9(3):235-7.

302. Crawford MW, Galton S, Naser B. Postoperative morphine consumption in children with sickle-cell disease. Paediatr Anaesth. 2006;16(2):152-7.

303. Chaplin H, Jr., Monroe MC, Malecek AC, Morgan LK, Michael J, Murphy WA. Preliminary trial of minidose heparin prophylaxis for painful sickle cell crises. East Afr Med J. 1989;66(9):574-84.

304. Dinges DF, Whitehouse WG, Orne EC, Bloom PB, Carlin MM, Bauer NK, et al. Self-hypnosis training as an adjunctive treatment in the management of pain associated with sickle cell disease. Int J Clin Exp Hypn. 1997;45(4):417-32.

305. Gill KM, Porter L, Ready J, Workman E, Sedway J, Anthony KK. Pain in children and adolescents with sickle cell disease: An analysis of daily pain diaries. Child Health Care. 2000;29(4):225-41.

306. Porter LS, Gil KM, Sedway JA, Ready J, Workman E, Thompson RJ, Jr. Pain and stress in sickle cell disease: an analysis of daily pain records. Int J Behav Med. 1998;5(3):185-203. 
307. Diggs LW. Bone and joint lesions in sickle-cell disease. Clin Orthop Relat Res. 1967;52:11943.

308. Ballas SK, Talacki CA, Rao VM, Steiner RM. The prevalence of avascular necrosis in sickle cell anemia: correlation with alpha-thalassemia. Hemoglobin. 1989;13(7-8):649-55.

309. Milner PF, Kraus AP, Sebes JI, Sleeper LA, Dukes KA, Embury SH, et al. Sickle cell disease as a cause of osteonecrosis of the femoral head. N Engl J Med. 1991;325(21):1476-81.

310. Amanatullah DF, Strauss EJ, Di Cesare PE. Current management options for osteonecrosis of the femoral head: part II, operative management. Am J Orthop (Belle Mead NJ). 2011;40(10):E216-25.

311. Ficat RP. Treatment of avascular necrosis of the femoral head. In: Hungerford DS, editor. The Hip. St. Louis, MO: Mosby; 1983. p. 279-95.

312. Steinberg ME, Hayken GD, Steinberg DR. A quantitative system for staging avascular necrosis. J Bone Joint Surg Br. 1995;77(1):34-41.

313. Steinberg ME, Steinberg DR. Classification systems for osteonecrosis: an overview. Orthop Clin North Am. 2004;35(3):273-83, vii-viii.

314. Amanatullah DF, Strauss EJ, Di Cesare PE. Current management options for osteonecrosis of the femoral head: part I, diagnosis and nonoperative management. Am J Orthop (Belle Mead NJ). 2011;40(9):E186-92.

315. Ficat RP. Idiopathic bone necrosis of the femoral head. Early diagnosis and treatment. J Bone Joint Surg Br. 1985;67(1):3-9.

316. Neumayr LD, Aguilar C, Earles AN, Jergesen HE, Haberkern CM, Kammen BF, et al. Physical therapy alone compared with core decompression and physical therapy for femoral head osteonecrosis in sickle cell disease. Results of a multicenter study at a mean of three years after treatment. J Bone Joint Surg Am. 2006;88(12):2573-82.

317. Mukisi-Mukaza M, Manicom O, Alexis C, Bashoun K, Donkerwolcke M, Burny F. Treatment of sickle cell disease's hip necrosis by core decompression: a prospective case-control study. Orthop Traumatol Surg Res. 2009;95(7):498-504.

318. Ebong WW. Avascular necrosis of the femoral head associated with haemoglobinopathy. Trop Geogr Med. 1977;29(1):19-23.

319. Ebong WW, Kolawole TM. Aseptic necrosis of the femoral head in sickle-cell disease. $B r J$ Rheumatol. 1986;25(1):34-9.

320. Mason VR. Sickle cell anemia. J Am Med Assoc. 1922;79:1318-20.

321. Koshy M, Entsuah R, Koranda A, Kraus AP, Johnson R, Bellvue R, et al. Leg ulcers in patients with sickle cell disease. Blood. 1989;74(4):1403-8.

322. Powars DR, Chan L, Schroeder WA. Beta S-gene-cluster haplotypes in sickle cell anemia: clinical implications. Am J Pediatr Hematol Oncol. 1990;12(3):367-74. 
323. Serjeant GR. Leg ulceration in sickle cell anemia. Arch Intern Med. 1974;133(4):690-4.

324. De Castro LM, Jonassaint JC, Graham FL, Ashley-Koch A, Telen MJ. Pulmonary hypertension associated with sickle cell disease: clinical and laboratory endpoints and disease outcomes. $\mathrm{Am}$ J Hematol. 2008;83(1):19-25.

325. Mack AK, Kato GJ. Sickle cell disease and nitric oxide: a paradigm shift? Int J Biochem Cell Biol. 2006;38(8):1237-43.

326. Nolan VG, Adewoye A, Baldwin C, Wang L, Ma Q, Wyszynski DF, et al. Sickle cell leg ulcers: associations with haemolysis and SNPs in Klotho, TEK and genes of the TGFbeta/BMP pathway. Br J Haematol. 2006;133(5):570-8.

327. Wethers DL, Ramirez GM, Koshy M, Steinberg MH, Phillips G, Jr., Siegel RS, et al. Accelerated healing of chronic sickle-cell leg ulcers treated with RGD peptide matrix. RGD Study Group. Blood. 1994;84(6):1775-9.

328. McMahon L, Tamary H, Askin M, Adams-Graves P, Eberhardt RT, Sutton M, et al. A randomized phase II trial of Arginine Butyrate with standard local therapy in refractory sickle cell leg ulcers. Br J Haematol. 2010;151(5):516-24.

329. Baum KF, MacFarlane DE, Maude GH, Serjeant GR. Topical antibiotics in chronic sickle cell leg ulcers. Trans R Soc Trop Med Hyg. 1987;81(5):847-9.

330. La Grenade L, Thomas PW, Serjeant GR. A randomized controlled trial of solcoseryl and duoderm in chronic sickle-cell ulcers. West Indian Med J. 1993;42(3):121-3.

331. Serjeant BE, Harris J, Thomas P, Serjeant GR. Propionyl-L-carnitine in chronic leg ulcers of homozygous sickle cell disease: a pilot study. J Am Acad Dermatol. 1997;37(3 Pt 1):491-3.

332. Okany CC, Atimomo CE, Akinyanju OO. Efficacy of natural honey in the healing of leg ulcers in sickle cell anaemia. Niger Postgrad Med J. 2004;11(3):179-81.

333. Serjeant GR, Galloway RE, Gueri MC. Oral zinc sulphate in sickle-cell ulcers. Lancet. 1970;2(7679):891-2.

334. Cackovic M, Chung C, Bolton LL, Kerstein MD. Leg ulceration in the sickle cell patient. J Am Coll Surg. 1998;187(3):307-9.

335. Cacciola E, Giustolisi R, Musso R, Longo A, Cacciola E. Antithrombin III concentrate for treatment of chronic leg ulcers in sickle cell-beta thalassemia: a pilot study. Ann Intern Med. 1989;111(6):534-6.

336. Aliyu ZY, Kato GJ, Taylor J 6th, Babadoko A, Mamman AI, Gordeuk VR, et al. Sickle cell disease and pulmonary hypertension in Africa: a global perspective and review of epidemiology, pathophysiology, and management. Am J Hematol. 2008;83(1):63-70.

337. Escribano Subias P, Barbera Mir JA, Suberviola V. Current diagnostic and prognostic assessment of pulmonary Hypertension. Rev Esp Cardiol. 2010;63(5):583-96. 
338. Ataga KI, Moore CG, Jones S, Olajide O, Strayhorn D, Hinderliter A, et al. Pulmonary hypertension in patients with sickle cell disease: a longitudinal study. Br J Hematol. 2006;134(1):109-15.

339. Barst RJ, Mubarak KK, Machado RF, Ataga KI, Benza RL, Castro O, et al. Exercise capacity and haemodynamics in patients with sickle cell disease with pulmonary hypertension treated with bosentan: results of the ASSET studies. Br J Haematol. 2010;149(3):426-35.

340. Machado RF, Martyr S, Kato GJ, Barst RJ, Anthi A, Robinson MR, et al. Sildenafil therapy in patients with sickle cell disease and pulmonary hypertension. Br J Haematol. 2005;130(3):44553.

341. Morris CR, Morris SM, Jr., Hagar W, Van Warmerdam J, Claster S, Kepka-Lenhart D, et al. Arginine therapy: a new treatment for pulmonary hypertension in sickle cell disease? Am J Respir Crit Care Med. 2003;168(1):63-9.

342. El-Beshlawy A, Abd El Raouf E, Mostafa F, Talaat M, Isma'eel H, Aoun E, et al. Diastolic dysfunction and pulmonary hypertension in sickle cell anemia: is there a role for L-carnitine treatment? Acta Haematol. 2006;115(1-2):91-6.

343. Pashankar FD, Carbonella J, Bazzy-Asaad A, Friedman A. Longitudinal follow up of elevated pulmonary artery pressures in children with sickle cell disease. Br J Haematol. 2009;144(5):736-41.

344. Levey AS, Eckardt KU, Tsukamoto Y, Levin A, Coresh J, Rossert J, et al. Definition and classification of chronic kidney disease: a position statement from Kidney Disease: Improving Global Outcomes (KDIGO). Kidney Int. 2005;67(6):2089-100.

345. Ataga KI, Orringer EP. Renal abnormalities in sickle cell disease. Am J Hematol. 2000;63(4):205-11.

346. Powars DR, Elliott-Mills DD, Chan L, Niland J, Hiti AL, Opas LM, et al. Chronic renal failure in sickle cell disease: risk factors, clinical course, and mortality. Ann Intern Med. 1991;115(8):614-20.

347. Falk RJ, Scheinman J, Phillips G, Orringer E, Johnson A, Jennette JC. Prevalence and pathologic features of sickle cell nephropathy and response to inhibition of angiotensinconverting enzyme. $N$ Engl J Med. 1992;326(14):910-5.

348. Guasch A, Navarrete J, Nass K, Zayas CF. Glomerular involvement in adults with sickle cell hemoglobinopathies: prevalence and clinical correlates of progressive renal failure. $J$ Am Soc Nephrol. 2006;17(8):2228-35.

349. Becton LJ, Kalpatthi RV, Rackoff E, Disco D, Orak JK, Jackson SM, et al. Prevalence and clinical correlates of microalbuminuria in children with sickle cell disease. Pediatr Nephrol. 2010;25(8):1505-11.

350. Ataga KI, Brittain JE, Moore D, Jones SK, Hulkower B, Strayhorn D, et al. Urinary albumin excretion is associated with pulmonary hypertension in sickle cell disease: potential role of soluble fms-like tyrosine kinase-1. Eur J Haematol. 2010;85(3):257-63. 
351. Francis YF, Worthen HG. Hyposthenuria in sickle cell disease. J Natl Med Assoc. 1968;60(4):266-70.

352. Field JJ, Austin PF, An P, Yan Y, DeBaun MR. Enuresis is a common and persistent problem among children and young adults with sickle cell anemia. Urology. 2008;72(1):81-4.

353. McCall IW, Moule N, Desai P, Serjeant GR. Urographic findings in homozygous sickle cell disease. Radiology. 1978;126(1):99-104.

354. Maigne G, Ferlicot S, Galacteros F, Belenfant X, Ulinski T, Niaudet P, et al. Glomerular lesions in patients with sickle cell disease. Medicine (Baltimore). 2010;89(1):18-27.

355. McKie KT, Hanevold CD, Hernandez C, Waller JL, Ortiz L, McKie KM. Prevalence, prevention, and treatment of microalbuminuria and proteinuria in children with sickle cell disease. J Pediatr Hematol Oncol. 2007;29(3):140-4.

356. Clarkson JG. The ocular manifestations of sickle-cell disease: a prevalence and natural history study. Trans Am Ophthalmol Soc. 1992;90:481-504.

357. Emerson GG, Lutty GA. Effects of sickle cell disease on the eye: clinical features and treatment. Hematol Oncol Clin North Am. 2005;19(5):957-73, ix.

358. Elagouz M, Jyothi S, Gupta B, Sivaprasad S. Sickle cell disease and the eye: old and new concepts. Surv Ophthalmol. 2010;55(4):359-77.

359. Goldberg MF. Classification and pathogenesis of proliferative sickle retinopathy. Am J Ophthalmol. 1971;71(3):649-65.

360. Dana MR, Werner MS, Viana MA, Shapiro MJ. Spontaneous and traumatic vitreous hemorrhage. Ophthalmology. 1993;100(9):1377-83.

361. Fox PD, Minninger K, Forshaw ML, Vessey SJ, Morris JS, Serjeant GR. Laser photocoagulation for proliferative retinopathy in sickle haemoglobin $\mathrm{C}$ disease. Eye (Lond). 1993;7 ( Pt 5):703-6.

362. Williamson TH, Rajput R, Laidlaw DA, Mokete B. Vitreoretinal management of the complications of sickle cell retinopathy by observation or pars plana vitrectomy. Eye (Lond). 2009;23(6):1314-20.

363. Kimmel AS, Magargal LE, Stephens RF, Cruess AF. Peripheral circumferential retinal scatter photocoagulation for the treatment of proliferative sickle retinopathy. An update.

Ophthalmology. 1986;93(11):1429-34.

364. Sayag D, Binaghi M, Souied EH, Querques G, Galacteros F, Coscas G, et al. Retinal photocoagulation for proliferative sickle cell retinopathy: a prospective clinical trial with new sea fan classification. Eur J Ophthalmol. 2008;18(2):248-54.

365. Latremoliere A, Woolf CJ. Central sensitization: a generator of pain hypersensitivity by central neural plasticity. J Pain. 2009;10(9):895-926.

366. Powars DR, Weiss JN, Chan LS, Schroeder WA. Is there a threshold level of fetal hemoglobin that ameliorates morbidity in sickle cell anemia? Blood. 1984;63(4):921-6. 
367. DeSimone J, Heller P, Hall L, Zwiers D. 5-Azacytidine stimulates fetal hemoglobin synthesis in anemic baboons. Proc Natl Acad Sci USA. 1982;79(14):4428-31.

368. Platt OS, Orkin SH, Dover G, Beardsley GP, Miller B, Nathan DG. Hydroxyurea enhances fetal hemoglobin production in sickle cell anemia. J Clin Invest. 1984;74(2):652-6.

369. Rodgers GP, Dover GJ, Noguchi CT, Schechter AN, Nienhuis AW. Hematologic responses of patients with sickle cell disease to treatment with hydroxyurea. $N$ Engl J Med. 1990;322(15):1037-45.

370. Charache S, Dover GJ, Moore RD, Eckert S, Ballas SK, Koshy M, et al. Hydroxyurea: effects on hemoglobin F production in patients with sickle cell anemia. Blood. 1992;79(10):2555-65.

371. Charache S, Terrin ML, Moore RD, Dover GJ, McMahon RP, Barton FB, et al. Design of the multicenter study of hydroxyurea in sickle cell anemia. Investigators of the Multicenter Study of Hydroxyurea. Control Clin Trials. 1995;16(6):432-46.

372. Ware RE. How I use hydroxyurea to treat young patients with sickle cell anemia. Blood. 2010;115(26):5300-11.

373. Charache S, Terrin ML, Moore RD, Dover GJ, Barton FB, Eckert SV, et al. Effect of hydroxyurea on the frequency of painful crises in sickle cell anemia. Investigators of the Multicenter Study of Hydroxyurea in Sickle Cell Anemia. N Engl J Med. 1995;332(20):131722 .

374. Steinberg MH, Barton F, Castro O, Pegelow CH, Ballas SK, Kutlar A, et al. Effect of hydroxyurea on mortality and morbidity in adult sickle cell anemia: risks and benefits up to 9 years of treatment. JAMA. 2003;289(13):1645-51. [Erratum appears in JAMA. 2003 Aug $13 ; 290(6): 756]$.

375. Steinberg MH, McCarthy WF, Castro O, Ballas SK, Armstrong FD, Smith W, et al. The risks and benefits of long-term use of hydroxyurea in sickle cell anemia: A 17.5 year follow-up. Am J Hematol. 2010;85(6):403-8.

376. Voskaridou E, Christoulas D, Bilalis A, Plata E, Varvagiannis K, Stamatopoulos G, et al. The effect of prolonged administration of hydroxyurea on morbidity and mortality in adult patients with sickle cell syndromes: results of a 17-year, single-center trial (LaSHS). Blood. 2010;115(12):2354-63.

377. Kinney TR, Helms RW, O'Branski EE, Ohene-Frempong K, Wang W, Daeschner C, et al. Safety of hydroxyurea in children with sickle cell anemia: results of the HUG-KIDS study, a phase I/II trial. Pediatric Hydroxyurea Group. Blood. 1999;94(5):1550-4.

378. Wang WC, Wynn LW, Rogers ZR, Scott JP, Lane PA, Ware RE. A two-year pilot trial of hydroxyurea in very young children with sickle-cell anemia. J Pediatr. 2001;139(6):790-6.

379. National Heart, Lung, and Blood Institute. Hydroxyurea to prevent organ damage in children with sickle cell anemia. In: ClinicalTrials.gov [Internet]. Bethesda (MD): National Library of Medicine (US). 2000 [cited 2013 Feb 8]. Available from: http://clinicaltrials.gov/show/NCT00006400. 
380. Wang WC, Ware RE, Miller ST, Iyer RV, Casella JF, Minniti CP, et al. Hydroxycarbamide in very young children with sickle-cell anaemia: a multicentre, randomised, controlled trial (BABY HUG). Lancet. 2011;377(9778):1663-72.

381. Zimmerman SA, Schultz WH, Davis JS, Pickens CV, Mortier NA, Howard TA, et al. Sustained long-term hematologic efficacy of hydroxyurea at maximum tolerated dose in children with sickle cell disease. Blood. 2004;103(6):2039-45.

382. Ballas SK, McCarthy WF, Guo N, DeCastro L, Bellevue R, Barton BA, et al. Exposure to hydroxyurea and pregnancy outcomes in patients with sickle cell anemia. J Natl Med Assoc. 2009;101(10):1046-51.

383. Flanagan JM, Howard TA, Mortier N, Avlasevich SL, Smeltzer MP, Wu S, et al. Assessment of genotoxicity associated with hydroxyurea therapy in children with sickle cell anemia. Mutat Res. 2010;698(1-2):38-42.

384. McGann PT, Howard TA, Flanagan JM, Lahti JM, Ware RE. Chromosome damage and repair in children with sickle cell anaemia and long-term hydroxycarbamide exposure. Br J Haematol. 2011;154(1):134-40.

385. Ferster A, Vermylen C, Cornu G, Buyse M, Corazza F, Devalck C, et al. Hydroxyurea for treatment of severe sickle cell anemia: a pediatric clinical trial. Blood. 1996;88(6):1960-4.

386. Wang W, Brugnara C, Snyder C, Wynn L, Rogers Z, Kalinyak K, et al. The effects of hydroxycarbamide and magnesium on haemoglobin SC disease: results of the multi-centre CHAMPS trial. Br J Haematol. 2011;152(6):771-6.

387. Hankins J, Hinds P, Day S, Carroll Y, Li CS, Garvie P, et al. Therapy preference and decisionmaking among patients with severe sickle cell anemia and their families. Pediatr Blood Cancer. 2007;48(7):705-10.

388. Anderson W, Ware R. Sickle cell anemia. J Am Med Assoc. 1932;99(11):902-5.

389. Aygun B, Padmanabhan S, Paley C, Chandrasekaran V. Clinical significance of RBC alloantibodies and autoantibodies in sickle cell patients who received transfusions. Transfusion. 2002;42(1):37-43.

390. Karafin MS, Shirey RS, Ness PM, King KE. Antigen-matched red blood cell transfusions for patients with sickle cell disease at The Johns Hopkins Hospital. Immunohematology. 2012;28(1):3-6.

391. Sloan SR. Transfusions for patients with sickle cell disease at Children's Hospital Boston. Immunohematology. 2012;28(1):17-9.

392. Winkler AM, Josephson CD. Transfusion practices for patients with sickle cell disease at major academic medical centers participating in the Atlanta Sickle Cell Consortium. Immunohematology. 2012;28(1):24-6.

393. Murphy RJ, Malhotra C, Sweet AY. Death following an exchange transfusion with hemoglobin SC blood. J Pediatr. 1980;96(1):110-2. 
394. Novak RW, Brown RE. Multiple renal and splenic infarctions in a neonate following transfusion with sickle trait blood. Clin Pediatr (Phila). 1982;21(4):239-41.

395. Whitaker B, Green J, King M, Leibeg L, Mathew S, Schlumpf K, et al. The 2007 national blood collection and utilization survey report. Washington, DC: U.S. Department of Health and Human Services, Office of the Assistant Secretary for Health, Office of Public Health and Science, 2007.

396. King KE, Shirey RS, Thoman SK, Bensen-Kennedy D, Tanz WS, Ness PM. Universal leukoreduction decreases the incidence of febrile nonhemolytic transfusion reactions to RBCs. Transfusion. 2004;44(1):25-9.

397. Bowden RA, Slichter SJ, Sayers M, Weisdorf D, Cays M, Schoch G, et al. A comparison of filtered leukocyte-reduced and cytomegalovirus (CMV) seronegative blood products for the prevention of transfusion-associated CMV infection after marrow transplant. Blood. 1995;86(9):3598-603.

398. The Trial to Reduce Alloimmunization to Platelets Study Group. Leukocyte reduction and ultraviolet B irradiation of platelets to prevent alloimmunization and refractoriness to platelet transfusions. N Engl J Med. 1997;337(26):1861-9.

399. Smith-Whitley K, Thompson AA. Indications and complications of transfusions in sickle cell disease. Pediatr Blood Cancer. 2012;59(2):358-64.

400. Koshy M, Weiner SJ, Miller ST, Sleeper LA, Vichinsky E, Brown AK, et al. Surgery and anesthesia in sickle cell disease. Cooperative Study of Sickle Cell Diseases. Blood. 1995;86(10):3676-84.

401. Howard J, Malfroy M, Llewelyn C, Choo L, Hodge R, Johnson T, et al. The transfusion alternatives preoperatively in sickle cell disease (TAPS) study: a randomised, controlled, multicentre clinical trial. Lancet. 2013;381(9870):930-8.

402. Vichinsky EP, Haberkern CM, Neumayr L, Earles AN, Black D, Koshy M, et al. A comparison of conservative and aggressive transfusion regimens in the perioperative management of sickle cell disease. The Preoperative Transfusion in Sickle Cell Disease Study Group. N Engl J Med. 1995;333(4):206-13.

403. Al-Samak ZM, Al-Falaki MM, Pasha AA. Assessment of perioperative transfusion therapy and complications in sickle cell disease patients undergoing surgery. Middle East J Anesthesiol. 2008;19(5):983-95.

404. Wali YA, al Okbi H, al Abri R. A comparison of two transfusion regimens in the perioperative management of children with sickle cell disease undergoing adenotonsillectomy. Pediatr Hematol Oncol. 2003;20(1):7-13.

405. Koshy M, Burd L, Wallace D, Moawad A, Baron J. Prophylactic red-cell transfusions in pregnant patients with sickle cell disease. A randomized cooperative study. $N$ Engl J Med. 1988;319(22):1447-52. 
406. Vichinsky EP, Luban NL, Wright E, Olivieri N, Driscoll C, Pegelow CH, et al. Prospective $\mathrm{RBC}$ phenotype matching in a stroke-prevention trial in sickle cell anemia: a multicenter transfusion trial. Transfusion. 2001;41(9):1086-92.

407. Napolitano LM, Kurek S, Luchette FA, Corwin HL, Barie PS, Tisherman SA, et al. Clinical practice guideline: red blood cell transfusion in adult trauma and critical care. Crit Care Med. 2009;37(12):3124-57.

408. Miller ST, Wright E, Abboud M, Berman B, Files B, Scher CD, et al. Impact of chronic transfusion on incidence of pain and acute chest syndrome during the Stroke Prevention Trial (STOP) in sickle-cell anemia. J Pediatr. 2001;139(6):785-9.

409. Mirre E, Brousse V, Berteloot L, Lambot-Juhan K, Verlhac S, Boulat C, et al. Feasibility and efficacy of chronic transfusion for stroke prevention in children with sickle cell disease. Eur $J$ Haematol. 2010;84(3):259-65.

410. Tahhan HR, Holbrook CT, Braddy LR, Brewer LD, Christie JD. Antigen-matched donor blood in the transfusion management of patients with sickle cell disease. Transfusion. 1994;34(7):562-9.

411. Vichinsky EP, Earles A, Johnson RA, Hoag MS, Williams A, Lubin B. Alloimmunization in sickle cell anemia and transfusion of racially unmatched blood. $N$ Engl J Med. 1990;322(23):1617-21.

412. Karam LB, Disco D, Jackson SM, Lewin D, McKie V, Baker RD, et al. Liver biopsy results in patients with sickle cell disease on chronic transfusions: poor correlation with ferritin levels. Pediatr Blood Cancer. 2008;50(1):62-5.

413. Angelucci E, Barosi G, Camaschella C, Cappellini MD, Cazzola M, Galanello R, et al. Italian Society of Hematology practice guidelines for the management of iron overload in thalassemia major and related disorders. Haematologica. 2008;93(5):741-52.

414. Cappellini MD, Porter J, El-Beshlawy A, Li C-K, Seymour JF, Elalfy M, et al. Tailoring iron chelation by iron intake and serum ferritin: the prospective EPIC study of deferasirox in 1744 patients with transfusion-dependent anemias. Haematologica. 2010;95(4):557-66.

415. Voskaridou E, Douskou M, Terpos E, Stamoulakatou A, Meletis J, Ourailidis A, et al. Deferiprone as an oral iron chelator in sickle cell disease. Ann Hematol. 2005;84(7):434-40.

416. Cancado R, Olivato MCA, Bruniera P, Chiattone C. Deferasirox for the treatment of transfusional iron overload in sickle cell anemia: a 1-yr prospective study. Haematologica. 2009;94:83.

417. Levin TL, Sheth SS, Hurlet A, Comerci SC, Ruzal-Shapiro C, Piomelli S, et al. MR marrow signs of iron overload in transfusion-dependent patients with sickle cell disease. Pediatr Radiol. 1995;25(8):614-9.

418. Ghoti H, Goitein O, Koren A, Levin C, Kushnir T, Rachmilewitz E, et al. No evidence for myocardial iron overload and free iron species in multitransfused patients with sickle/betathalassaemia. Eur J Haematol. 2010;84(1):59-63. 
419. Vichinsky E, Onyekwere O, Porter J, Swerdlow P, Eckman J, Lane P, et al. A randomised comparison of deferasirox versus deferoxamine for the treatment of transfusional iron overload in sickle cell disease. Br J Haematol. 2007;136(3):501-8.

420. Fung EB, Harmatz PR, Milet M, Balasa V, Ballas SK, Casella JF, et al. Disparity in the management of iron overload between patients with sickle cell disease and thalassemia who received transfusions. Transfusion. 2008;48(9):1971-80.

421. Ballas SK. Iron overload is a determinant of morbidity and mortality in adult patients with sickle cell disease. Semin Hematol. 2001;38(1 Suppl 1):30-6.

422. Harmatz P, Butensky E, Quirolo K, Williams R, Ferrell L, Moyer T, et al. Severity of iron overload in patients with sickle cell disease receiving chronic red blood cell transfusion therapy. Blood. 2000;96(1):76-9.

423. de Montalembert M, Dumont MD, Heilbronner C, Brousse V, Charrara O, Pellegrino B, et al. Delayed hemolytic transfusion reaction in children with sickle cell disease. Haematologica. 2011;96(6):801-7.

424. Win N, New H, Lee E, de la Fuente J. Hyperhemolysis syndrome in sickle cell disease: case report (recurrent episode) and literature review. Transfusion. 2008;48(6):1231-8.

425. Haberkern CM, Neumayr LD, Orringer EP, Earles AN, Robertson SM, Black D, et al. Cholecystectomy in sickle cell anemia patients: perioperative outcome of 364 cases from the National Preoperative Transfusion Study. Preoperative Transfusion in Sickle Cell Disease Study Group. Blood. 1997;89(5):1533-42.

426. Vichinsky EP, Neumayr LD, Haberkern C, Earles AN, Eckman J, Koshy M, et al. The perioperative complication rate of orthopedic surgery in sickle cell disease: report of the National Sickle Cell Surgery Study Group. Am J Hematol. 1999;62(3):129-38.

427. Freed J, Talano J, Small T, Ricci A, Cairo MS. Allogeneic cellular and autologous stem cell therapy for sickle cell disease: 'whom, when and how'. Bone Marrow Transplant. 2012;47(12):1489-98.

428. Bolaños-Meade J, Fuchs EJ, Luznik L, Lanzkron SM, Gamper CJ, Jones RJ, et al. HLAhaploidentical bone marrow transplantation with posttransplant cyclophosphamide expands the donor pool for patients with sickle cell disease. Blood. 2012;120(22):4285-91. 
U.S. Department of Health and Human Services National Institutes of Health

National Heart, Lung, and Blood Institute 